\title{
Option markets : implied information, equity process and risk management
}

Citation for published version (APA):

Blanchard, G. Y. (2015). Option markets : implied information, equity process and risk management. [Doctoral Thesis, Maastricht University]. Datawyse / Universitaire Pers Maastricht. https://doi.org/10.26481/dis.20150211gb

Document status and date:

Published: 01/01/2015

DOI:

10.26481/dis.20150211gb

Document Version:

Publisher's PDF, also known as Version of record

\section{Please check the document version of this publication:}

- A submitted manuscript is the version of the article upon submission and before peer-review. There can be important differences between the submitted version and the official published version of record.

People interested in the research are advised to contact the author for the final version of the publication, or visit the DOI to the publisher's website.

- The final author version and the galley proof are versions of the publication after peer review.

- The final published version features the final layout of the paper including the volume, issue and page numbers.

Link to publication

\footnotetext{
General rights rights.

- You may freely distribute the URL identifying the publication in the public portal. please follow below link for the End User Agreement:

www.umlib.nl/taverne-license

Take down policy

If you believe that this document breaches copyright please contact us at:

repository@maastrichtuniversity.nl

providing details and we will investigate your claim.
}

Copyright and moral rights for the publications made accessible in the public portal are retained by the authors and/or other copyright owners and it is a condition of accessing publications that users recognise and abide by the legal requirements associated with these

- Users may download and print one copy of any publication from the public portal for the purpose of private study or research.

- You may not further distribute the material or use it for any profit-making activity or commercial gain

If the publication is distributed under the terms of Article $25 \mathrm{fa}$ of the Dutch Copyright Act, indicated by the "Taverne" license above, 


\section{Option Markets}

Implied Information, Equity Prices and

Risk Management

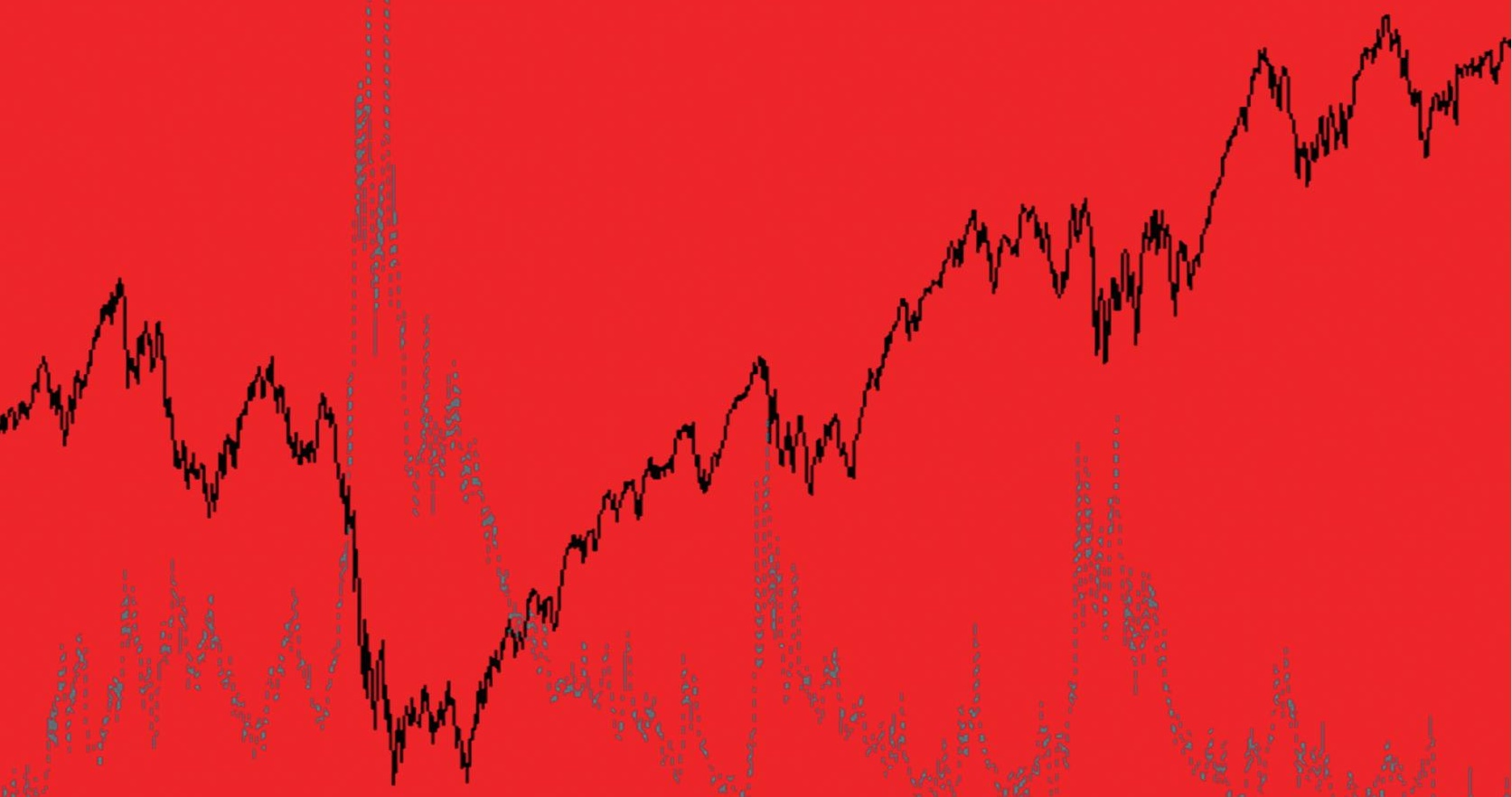

\section{Gildas Yannick Blanchard}


ISBN 9789461594082

(C) Copyright Gildas Yannick Blanchard, Maastricht 2015

Printing: Datawyse / Universitaire Pers Maastricht 


\title{
Option Markets: Implied Information, Equity Prices and Risk Management
}

\author{
DISSERTATION \\ to obtain the degree of Doctor at Maastricht University, \\ on the authority of the Rector Magnificus, \\ Prof. dr. L.L.G. Soete, \\ in accordance with the decision of the Board of Deans, \\ to be defended in public \\ on Wednesday, $11^{\text {th }}$ February 2015, at 16:00 hours
}

by

Gildas Yannick Blanchard

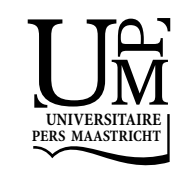




\section{Supervisors}

Prof. dr. Dennis Bams

Prof. dr. Thorsten Lehnert, Université du Luxembourg

\section{Assessment Committee}

Prof. dr. Peter Schotman, Chairman

Prof. dr. Jaap Bos

Prof. dr. Aline Muller, University of Liège

Prof. dr. Christian Wolff, Université du Luxembourg

This research has benefited from the financial support granted by the Graduate School of Business and Economics (GSBE). 
To My Wife 



\section{Table of contents}

List of Tables I

List of Figures

Acknowledgements $\quad$ V

Chapter 1 - Introduction $\quad 1$

1.1. Derivatives and options in human history........................................................ 1

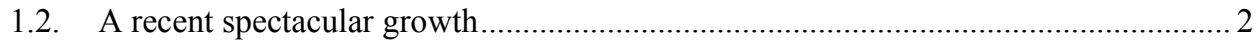

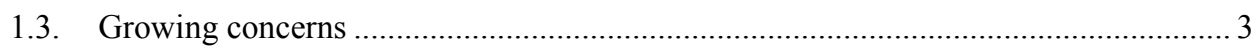

1.4. A rich and growing body of academic knowledge.............................................. 5

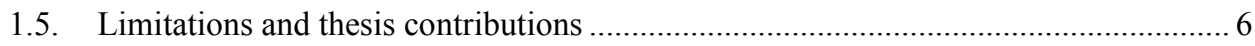

Chapter 2 - Evaluating Option Pricing Model Performance Using Model Uncertainty 11

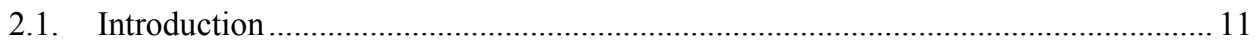

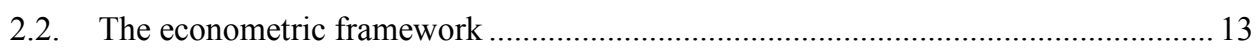

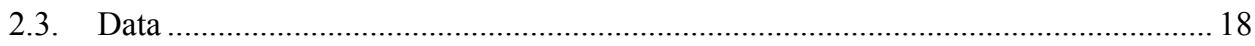

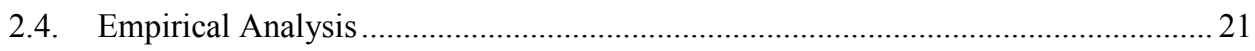

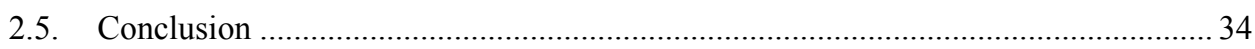

Chapter 3 - On the Impact of Exclusion Filters Rules in Option Pricing 37

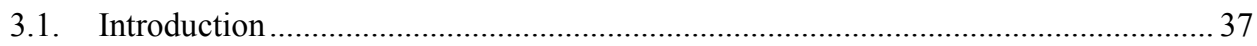

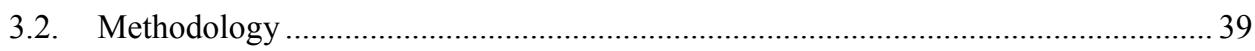

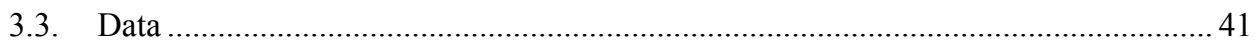

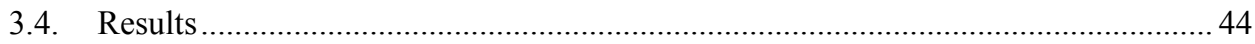

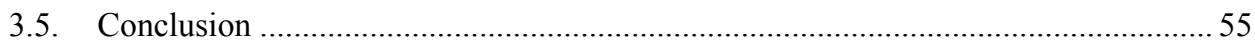

Chapter 4 - The Impact of Uncertainty in the Oil and Gold Market on the Cross-

Section of Stock Returns...................................................................................................5 59

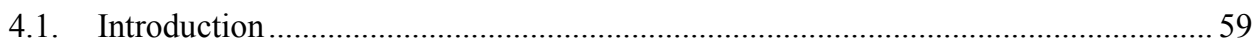

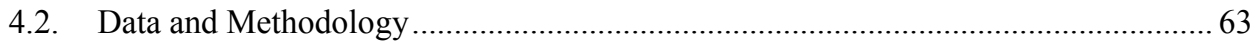

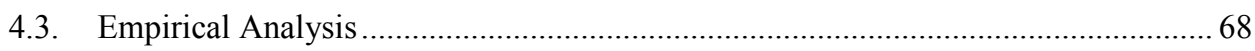

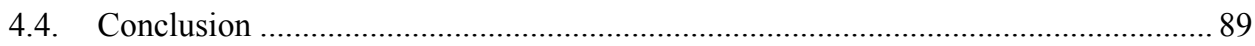

Chapter 5 - Forecasting Volatility or Value-at-Risk: when does the Volatility Risk

$\begin{array}{ll}\text { Premium matter? } & 91\end{array}$

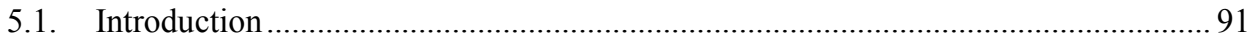

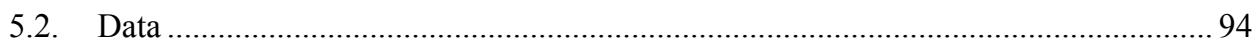

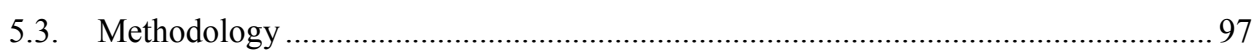

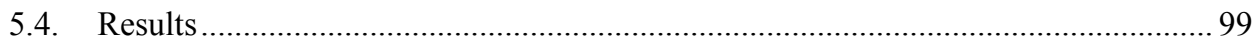

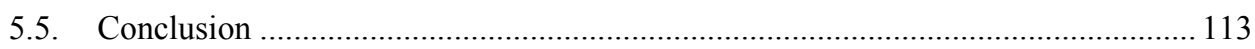


References

Summary

123

Addendum

127

Biography 


\section{List of Tables}

1.1: Synoptic view of the option pricing model literature review ..............................................5

2.1: Average price, BS-Implied volatility and number of contract by moneyness and maturity

2.2: Sample composition and market conditions descriptive statistics .....................................20

2.3: Parameter estimates and resulting in-sample RMSE ....................................................22

2.4: One sample T-test of the average observed price-average bootstrapped price difference per option category.........................................................................................................................23

2.5: Time series and bootstrap parameters information comparison ........................................24

2.6: Correlation matrix - time series and cross-section ........................................................... 26

2.7: RMSE distributions in different context- statistical test ................................................29

2.8: Cross-section pricing performance, sample composition and market conditions...............30

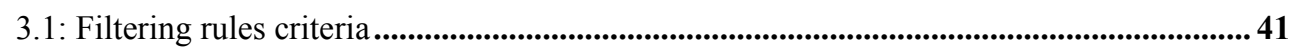

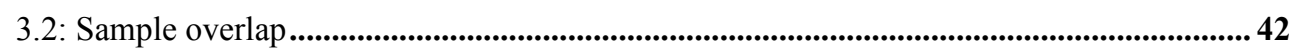

3.3: Descriptive statistics for each sample ...........................................................................44

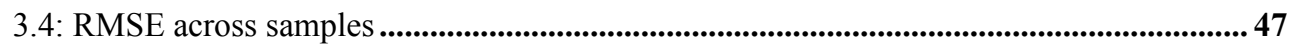

3.5: 1 Week out-of-sample RMSE across sample - bucket by bucket .....................................50

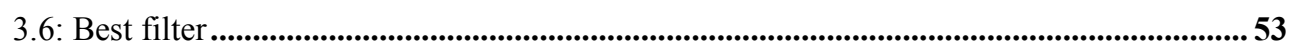

3.7: RMSE across sample maturity adjusted (out-sample pricing 1 week) ..............................55

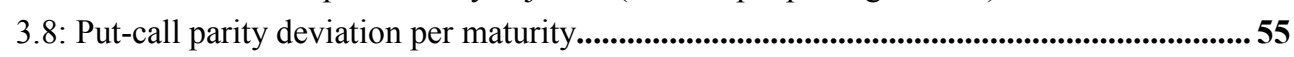

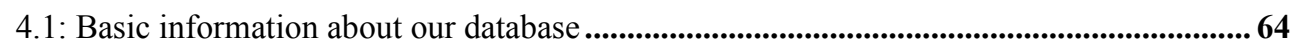

4.2: Descriptive statistics on volatility risk premia .................................................................71

4.3: Contemporaneous effect of uncertainty innovation on price of stocks ............................73

4.4: Controled contemporaneous effect of uncertainty on price of stocks..................................76

4.5: Expected return of cross-sectional exposure to uncertainty.............................................80

4.6: Expected return of cross-sectional exposure to uncertainty for small, medium and big

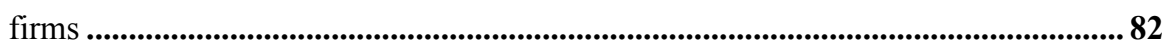

4.7: Expected return of cross-sectional exposure to uncertainty for expansion and recession

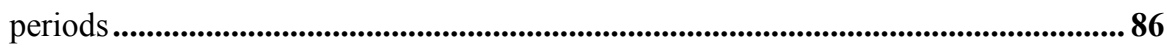

4.8: Expected return of cross-sectional exposure to uncertainty for different industries..........87

5.1: Implied volatility, realized volatility and volatility risk premium ...................................99

5.2: Summary volatility forecasts - time series versus implied volatility..................................99

5.3: Time series and implied volatilities based Value-at-Risk measures unconditional performances .................................................................................................................... 103

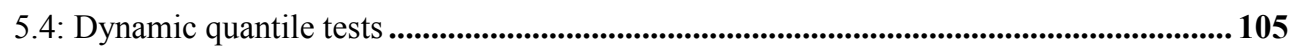

5.5: Loss function and loss function difference tests ........................................................ 107 
5.6: Time series and implied volatilities based monthly Value-at-Risk measures unconditional performances ..................................................................................................109

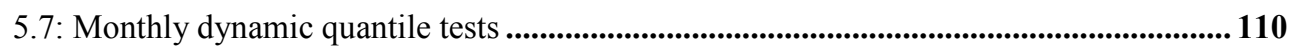

5.8: Monthly loss function and loss function difference tests ...........................................112 


\section{List of Figures}

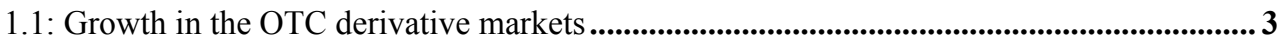

1.2: Top 45 trading losses per instruments.................................................................................. 4

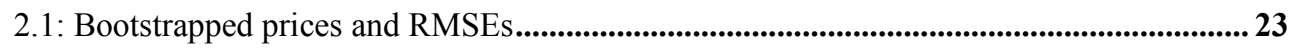

2.2: RMSE uncertainty represented as bootstrapped distribution in different contexts........... 28

2.3: Very inexpensive options and uncertainty ..............................................................31

2.4: Cross-sectional specification tests................................................................................................. 33

3.1: Distribution of observations per option categories per EFR .............................................45

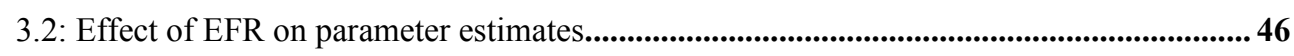

3.3: Put Vs Call residuals ......................................................................................................................5 57

4.1: Volatility risk premia of S\&P 500, oil and gold ..................................................................... 70

4.2: Expected return of cross-sectional exposure to uncertainty............................................83

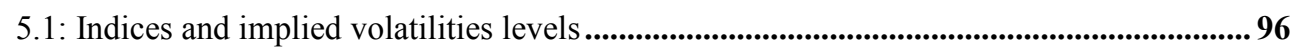

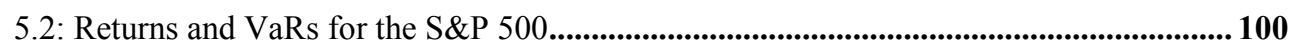





\section{Acknowledgements}

The doctoral degree route consists of two distinct components: scientific knowledge and human interaction. $99 \%$ of this book is dedicated to the former aspect. This is, in fact, an unfair representation of the reality since interactions, friendship and family are inherent and necessary parts of the accomplishment of a doctoral degree. Accordingly, I will now try to convey my gratitude towards those wonderful personalities that have surrounded, supported and helped me over the past four years.

Special thanks is owed to my supervisors Professor Dennis Bams and Professor Thorsten Lehnert. I thank both of you for giving me the opportunity of pursuing a doctoral degree. I am highly indebted towards you for giving me this scarce and life-changing experience. The trust you provided me from the day you accepted me as your doctoral student onwards is still a source of pride for me. Your devotion in sharing your knowledge, expertise and passion has helped me to hone my research abilities. You were also aware as to how best to advise, challenge, push and support me at the right time and the right moment. Without your guidance I would have probably not finished this $\mathrm{PhD}$. Thank you for all the $\mathrm{PhD}$ - (and non- $\mathrm{PhD}-$ ) related help you provided me with along these four years. I certainly look forward to sustaining our academic and non-academic relationship in the future years.

Pursuing doctoral studies is a rather uncommon fate for someone like me. But there are a few people that have played an instrumental role in turning on the little academic light bulb in my mind. Mr Charles Booth, you have conveyed with passion in the classroom you knowledge about the philosophy of science over generations of DBK students (Developing Business Knowledge). Science was a stranger to me before I arrived in Bristol and the idea of devoting 4 years of my life to it had never come to me prior to me taking your course. Dr Gregory Heem was the mentor and source of guidance of my first academic work. You made this process so smooth and enjoyable that I could only continue on the footsteps you helped me develop. Dr David Barr, you have introduced me to econometrics. My ignorance was bliss but you never seemed tired of filling it with your incredible knowledge. Dr Kallinterakis, you are the one who convinced me that I was a research material. You were even more convinced than me and you played an instrumental role in my choice. I cannot stress enough the importance of all the wonderful teachers who stimulated my curiosity over these last 9 years of studies. I would like to thank all the professors and educators I met at the Edhec Business 
School, at the University of the West of England, at the University of Durham and, naturally at the University of Maastricht.

I feel blessed for having been a part of the finance department for four years. The concentration and diversity of knowledge contained within two corridors is spectacular. My daily conversations with Prof Rob Bauer, Prof Jaap Bos, Dr Jeroen Derwall, Prof Piet Eichholtz, Dr Arvid Hoffmann, Prof Harry Hummels, Prof Georges Hübner, Dr Stefanie Kleimeier, Dr Nils Kok, Dr Roger Otten, Prof Joost Pennings, Dr Thomas Post, Prof Rachel Pownall, Dr Paulo Rodrigues, Prof Peter Schotman, Dr Stefan Straetmans have enlightened me on topics such as financial econometrics, art, risk management, asset pricing, real estate, pension funds, mutual funds, household finance, behavioural economics and scotch whiskies. I am grateful to the accessibility and the time these experts have given to me. I would like also to thank especially my assessment committee members: Prof Peter Schotman, Prof Jaap Bos, Prof Aline Muller and Prof Christian Wolff for the time they dedicated to my thesis. I am grateful for their invaluable comments and insights.

My students have always been a great source of inspiration to me. Their expectations have helped me to surpass myself as an educator. Their curiosity, knowledge and intelligence have helped me to question my own knowledge. Their appreciation and support have given me pride. I truly enjoyed every single of my teaching minutes thanks to my Master thesis students, my financial risk management students and my macroeconomics and finance students.

Four very special "Ladies", namely Franien, Cécile, Carina and Else, are everyday giving a whole meaning to the expression 'going the extra mile'. I would have probably not been able to survive in the Netherlands without your tax advices, letter translation services, car insurance provision and so much other invaluable help. But most importantly you made me feel at home with your welcoming smiles and never-failing cheerfulness. I cherish and already miss our daily talks. Marie-José I also miss our conversations. Thank you very much for your kindness and for your pep talks.

The difficulties and joys of the $\mathrm{PhD}$ process allow for the transformation of a bunch of strangers into a bonded group of true friends. The fact alone that my $\mathrm{PhD}$ allowed me to meet such wonderful friends among the $\mathrm{PhD}$ students rendered the $\mathrm{PhD}$ itself worth it.

I would like to thank you all for the drinks out, the lunch, the pep talks, the talks, the advices, the smiles, the coffees, the laughs, the Ardennes' week-ends, the thanksgiving dinners, the conference trips and every single minute I spent with you. Arian, Judy, Inka, Wiebke, Oana, Patrick, Clarissa, Rogier. H, Sjoke, Magdalena, Rogier. Q (special consultant for my Chapter 5), Sally, David, Bart, Daniel, Hang, Pomme, Carl, Anna, Andrea, Simone, Robert, Aleks, Lei, Sajid, Johannes and Marie you have been wonderful road companions but also much 
more. I have no doubt that our roads will cross not by hazard but because of my will to share more time with you. I wish I had more time to know better Alessandro, Tobias, Inke, Vincent and Eshan.

Some people have had the hard task of bearing me longer than others. My office mate for the entire four years has by far surpassed anyone in this role. Matteo: on top of being a consultant in almost everything (restaurant, movies, vacation venues and tailors), you are a great friend to be around. I look forward reading your papers on game economics. I am already missing my lunch and coffee filled with truth-revealing talks with Paul. Paul, thanks for reminding me at multiple occasions what mattered in life. I must also acknowledge the instrumental role of the so called "Musketeers of Maastricht" composed of Mukul, Omar, Iman and from now on Gaby for reminding me that the world and life itself are just a big playground. Our constant bantering, jokes and discussions are legendary anecdotes that still put a smile on my face while thinking about them. Thanks for having believed in me and encouraged me throughout the final stage of the $\mathrm{PhD}$. In one single night I have been blessed with the discovery of two things that mean a lot to me. First, the leffe double at Tribunal; second, an amazing and invaluable friendship. For both of these discoveries I have to thank Leonard.

The $\mathrm{PhD}$ roller coaster is made up of a lot of ups and downs. While the ups fill you with elation and euphoria, the downs reveal to you the extreme kindness and support that people are eager to offer you. I am in a way grateful to have experienced those moments allowing me to learn more about the best of human nature. Jeroen, Paulo, Michael, Stefanie and Nico, I thank you all for having me shown the light when it was the most needed.

Finally, my last words of gratitude are directed toward my "everything". This gratitude is not limited to the sphere and years of the $\mathrm{PhD}$. It has, is and will always be about my family. The love and unconditional support of my family has always been my safe haven. I would like to thank my parents. Papa, Maman you have made this achievement possible in so many ways. You have taught me curiosity, perseverance and it is perhaps fair to say that my educational achievements are rooted in the local motto "Gildas, travaille". But, first and foremost you helped and are helping me to cope with my fear of uncertainty by providing me the certainty of your love and presence. Your support, trust and love were instrumental to the achievement of this thesis and to the achievement of any project I am undertaking. Thanks for being there for me the last 28 years.

My sisters, brother and sister-in-law are forming my dream team. Aude, Luc, Ghislaine and Lydie, I thank you all for your unconditional support and the trust you gave me along the years. Being the Benjamin of the family I enjoy the particular attention and protection from my elder siblings, who were always extremely attentive to my need and happiness. Aude thanks for having been a little mother and a role model on what to do. Ghislaine thanks for always reminding me what happiness is about and thanks for having always been able to 
understand my twisted personality. Luc and Lydie thanks for taking me like a brother of your own. I would also like to thank my three little nephews and niece Baptiste, Morgan and Gautier who are my injection of energy, fun, love and craziness every time I see them.

It is of public knowledge that family is sacrosanct. Therefore, only the most exceptional and perfect lady could come and become my family. For the thesis, like for anything, you are my source of inspiration and motivation. You stood by me and by my black dog at any stage. You gave me faith or you had faith for me when I stopped believing it was possible. You provided me relentlessly with encouragement, moral and technical support. And when you did not know what to do, you stayed here holding my hand. This thesis would have never been accomplished without your daily love and strength. You are the most perfect and courageous wife. Clémentine, merci! 


\section{Chapter 1}

\section{Introduction}

\subsection{DERIVATIVES AND OPTIONS IN HUMAN HISTORY}

"According to the story, he knew by his skill in the stars while it was yet winter that there would be a great harvest of olives in the coming year; so, having little money, he gave deposits for the use of all olive-presses in Chios and Miletus, which he hired at a low price because no one bid against him. When the harvest-time came, and many wanted them all at once and of a sudden, he let them out at any rate which he pleased, and made a quantity of money" (Aristotle, 1885:21). This is the way Aristotle described the financial engineering skills displayed by 'He ', namely Thales of Miletus, in his famous literary work Politics. Although this anecdote involves multiple notions such as philosophy, meteorology and monopoly power, it is also seen by many as portraying the first example in history of a derivative instrument (Allende and Elias, 2004;Dimson and Mussavian, 1999; Cummins, Philips and Smith, 1998).

Indeed, with this design, Thales bought the right to rent the olive oil presses at a fixed price within a certain period. This description matches the definition of a call option. The buyer owns the right, but not the obligation, to buy (or sell) an asset for a certain price at a certain time. The deposit made by Thales can be seen as the premium for the contingent claim he owned (Makropoulou and Markellos, 2005). As in the case of any derivatives, the value of Thales' call option was derived from the value of an underlying. The raison d'etre of this option was the random characteristic of the underlying. The olive press rent price was random due to the olive crop uncertainty.

Although this story may seem a simple anecdote of a philosopher displaying his skills in the art of money-making, it conveys a much stronger message about options and derivative markets. Thales died in approximately $546 \mathrm{BC}$ and Aristotle is believed to have written Politics throughout his entire life until he died in 322 BC. This shows that despite the fact that options are popularly believed to be a new financial instrument, the intellectual concept and the use of derivatives have existed for a very long time (Capelle-Blancard, 2011). This very enduring interest in and understanding of options in history is explained by the inherent human need to deal with randomness. An option can be written on any random variable. It may be olive production, stock prices, commodity prices, interest rates, electricity prices or even rainfall. In 2002, following the recommendations of Shiller, Goldman Sach and 


\section{CHAPTER 1}

Deutsche Bank launched the first 'Economic Derivatives' written on underlyings such as initial unemployment claims (Gurkaynak and Wolfers, 2006). The option market is the market place where randomness is exchanged and priced. In a broader sense, randomness can be defined as uncertainty and risk.

Market participants are divided between speculators who want to take on risk and obtain compensation for this, and hedgers who desire to eliminate this risk. The option markets create theoretical market completeness and allow this transaction to occur efficiently. The intellectual development of derivatives goes hand in hand with the need to manage uncertainty and risk. Covello and Mumpower (1985) report the existence of some forms of risk consultancy in Mesopotamia back in 3200 B.C.

Options are intended to deal with one of the most enduring of human concerns. This is a sufficient motivation to investigate, to try to understand and to question ourselves on the dynamics and the evolution of option markets. Moreover, recent developments observed on these markets provide an even stronger incentive to study this.

\subsection{A RECENT SPECTACULAR GROWTH}

The recent history of derivatives markets is characterized by spectacular growth over the past decades. Figure 1.1 shows the evolution of the gross market value of Over-The-Counter (OTC) derivatives per underlying category. In 1998, the total gross market value amounted to slightly over $\$ 2,500$ billion. In 2013 this gross market value grew to more than $\$ 18,500$ billion. Another way to appreciate the size of derivatives markets is to evaluate it in terms of the notional amount outstanding. In 2013 this figure reached more than $\$ 710,000$ billion. By comparison, the 2013 world gross domestic product was, according to the World Bank, almost $\$ 75,000$ billion. The suggested causes of this phenomenal growth are twofold. It is argued that the development of mathematical and quantitative tools such as the Black and Scholes formula, in 1973, permitted the pricing of contingent claim and therefore its growth. In that sense, there are strong parallels between the insurance industry and the derivative markets.

The intellectual development of probability theory at the end of the Middle Ages (Covello and Mumpower, 1985; Bernstein, 1996) facilitated the birth of the insurance business. The development of pricing theories turned the option market into a fast growing, liquid and mature market. Theory and practice are intimately linked in the field of derivatives and option pricing. The growth of the derivative market has also been accompanied by the birth of a considerable number of academic peer reviewed journals specializing in the topic, such as The Journal of Derivatives, The Journal of Futures Markets, Journal of Derivative Research and Journal of Derivatives and Hedge Funds (Capelle-Blancard, 2011). Mixon (2009) argues that centralized exchanges played a greater role in the development of the option markets. $\mathrm{He}$ 
shows that the Chicago Board of Exchange market opening allowed more participants to reach the market and therefore reduced the compensation for risk bearing.

Figure 1.1: Growth in the OTC derivative markets

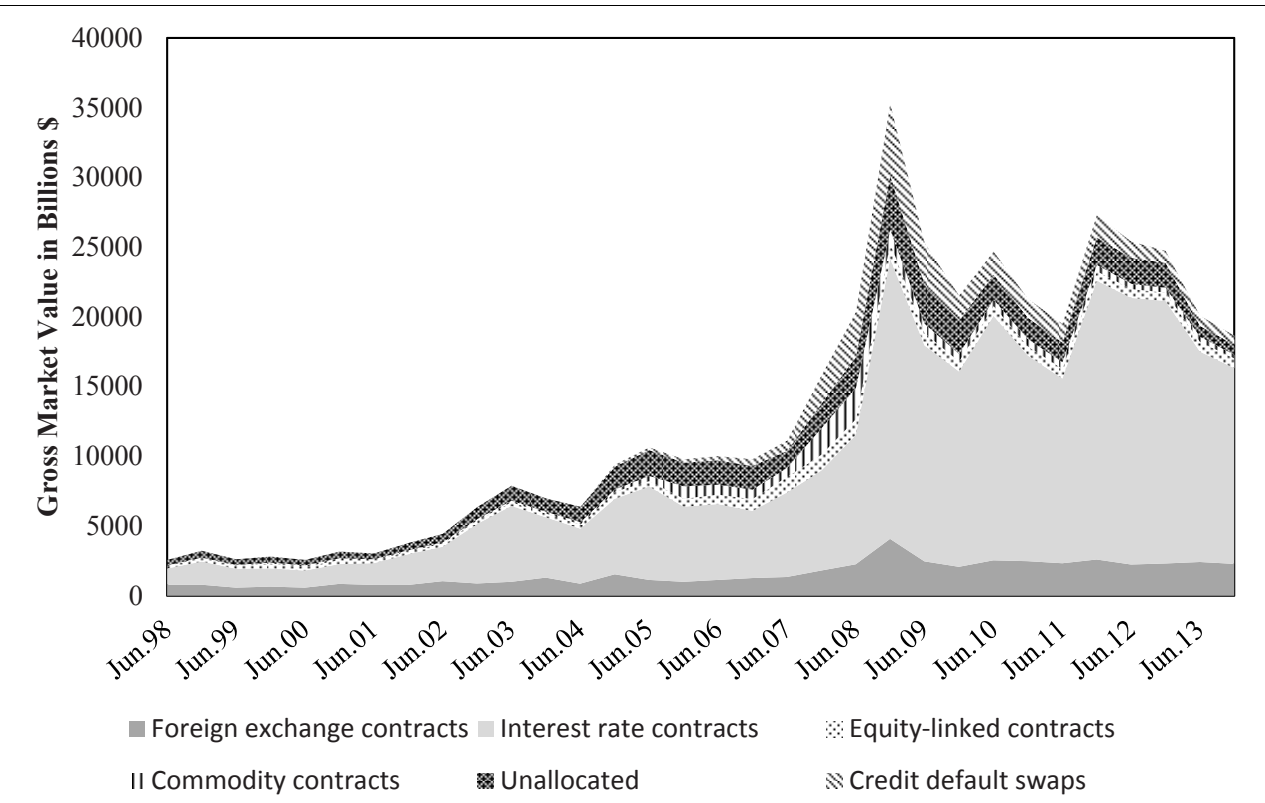

Notes: The data on the gross market value of the OTC derivatives markets has been collected from the OTC derivatives statistics webpage of the Bank of International Settlement website.

The most realistic explanation of this exceptional growth probably lies somewhere between the two views above. Both the consequences and the effects of the derivative market surge call for more research on options and derivatives related topics. The better understanding of derivatives has permitted the popularization of derivatives usage and the development of derivatives markets. Because of the systematic size of options and derivatives markets, we cannot be satisfied with a limited understanding of them.

\subsection{GROWING CONCERNS}

The benefits of the option markets are well-known. They facilitate an efficient allocation of risk amongst economic agents over a complete market. Derivatives are also found to reduce transaction costs, to improve both liquidity and the price discovery process (Easley et al., 1998). Furthermore, the usage of insurance and derivatives by non-financial corporations is found to be a source of productivity enhancement (Cornaggia, 2013) and value-creation for shareholders (Pérez-González and Yun, 2010; Bartram, Brown and Conrad, 2011). 


\section{CHAPTER 1}

However, the fear and severe criticisms nonetheless triggered by derivatives are also vivid examples and consequences of our lack of sufficient understanding of this market for innovations. The long term fundamental financial investment guru, Warren Buffet, described derivatives as "weapons of mass destruction". This opinion is extreme, but the political debate about the regulation of derivatives markets and derivatives usage is an obvious sign of public concern. Mixon (2009) reports that as far back as in 1874, writing option contracts on commodities, gold and stocks was made illegal in Illinois. Nowadays, total prohibition would seem nonsensical. But an attempt does exist to increase regulation of derivatives markets. The European Market Infrastructure Regulation (EMIR) is a perfect example of such an initiative.

The complexity, the speed of innovation and the systematic concerns have driven this recent movement on regulating the OTC markets. These three elements combined make risk management of derivatives trading an extremely complicated task. Derivatives trading is at the root of the biggest publically reported trading losses in history. Indeed, the non-academic website Wikipedia provides a list of the Top 45 trading losses. The cumulated losses amount to almost 82.5 billion current US dollars, where 65.5 billion current US dollars, or $79 \%$ of the total amount, are losses made on derivatives markets.

Figure 1.2 shows the distribution of the losses across products. The huge losses made on commodity derivatives result from a misunderstanding of the products, leading to fallacious hedging practices. The infamous case of Metallgesellschaft corporation illustrates this type of loss. Important losses can also be attributed to 'rogue traders', such as Nick Leeson or Jérome Kerviel, who claimed to be using the derivatives market for arbitrage and hedging purposes but were actually engaging in pure speculation.

Figure 1.2: Top 45 trading losses per instruments

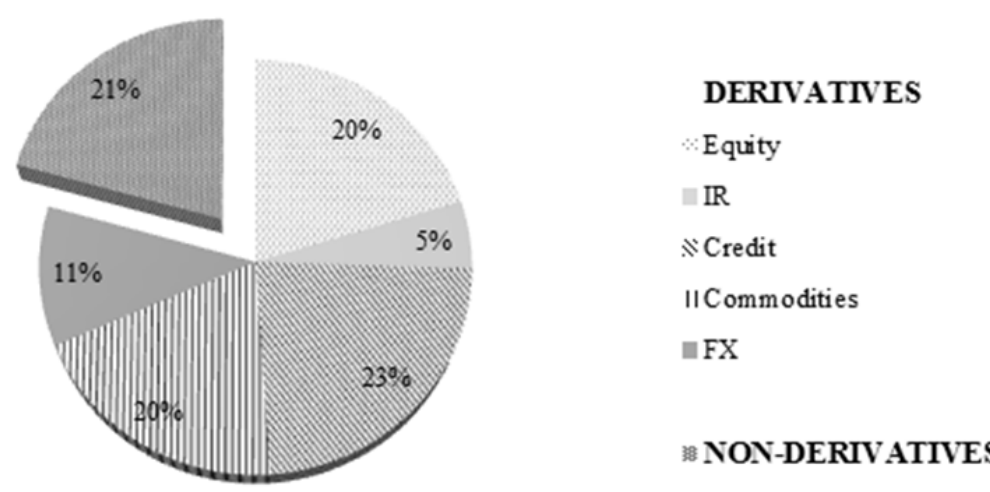

Notes: The list of losses is available on the Wikipedia page 'List of trading losses'. The individual loss amounts and the origin of the losses are also collected from the same source. When the origin of the loss is not reported, manual research is performed to allocate the loss to a certain segment. The individual and total loss amounts are converted to 2013US dollars in order to account for inflation. 
These statistics highlight the complexity, the rapid innovations and the dynamic characteristics of derivatives markets.

The inherent human preference for risk transfer, the overall importance of derivatives in the world economy and the dramatic economic consequences of derivative misuse are multiple reasons that make the creation of scientific knowledge about option markets a necessity. The first academic efforts directed towards this topic were on derivatives pricing. What is the price of a contingent claim? Unlike the existence of options, the ability to price options is relatively new. The first attempts to price contingent claims were made in the twentieth century by Bachelier and finally by Black and Scholes (Dimson and Mussavian, 1999). The Black and Scholes formula was a real scientific and economic breakthrough. Some even attribute the recent explosion of derivatives markets to the new pricing techniques introduced since Black and Scholes.

\subsection{A RICH AND GROWING BODY OF ACADEMIC KNOWLEDGE}

With the explosion of a liquid option market, the objective evolved from finding $a$ pricing formula to finding the optimal formula that matches closely theobserved option market prices. These investigations led to the realization that the Black and Scholes model does not properly

Table 1.1: Synoptic view of the option pricing model literature review

\begin{tabular}{lll}
\hline Model Family & Representative study & \\
\hline $\begin{array}{l}\text { Continuous Time Stochastic } \\
\text { Volatility }\end{array}$ & Heston (1993) & Stochastic Volatility \\
& $\begin{array}{l}\text { Phernov, Gallant, Ghysels and Tauchen } \\
\text { (2003) }\end{array}$ & $\begin{array}{l}\text { With volatility Jump correlated with } \\
\text { return jump }\end{array}$ \\
\hline $\begin{array}{lll}\text { Discrete Time Volatility } \\
\text { Duan (1995) }\end{array}$ & $\begin{array}{l}\text { Christoffersen and Jacobs (2004a) } \\
\text { Barone-Adesi, Engle and Mancini (2008) }\end{array}$ & $\begin{array}{l}\text { GJR Garch with non normal } \\
\text { innovations }\end{array}$ \\
\hline "Smile Consistent” model & $\begin{array}{l}\text { Dumas, Flemming and Whaley (1998); } \\
\text { Barle and Cakici (1995) }\end{array}$ & \begin{tabular}{l} 
Smoothed implied volatilities \\
\hline
\end{tabular} \\
\hline
\end{tabular}

describe option prices. The Black and Scholes model is notorious for its inability to match the volatility surface. The Black and Scholes option implied volatilities are varying with respect to time and moneyness. Both practitioners and academics have attempted to provide option pricing models able to fit this option implied volatility surface. The option pricing volatility 


\section{CHAPTER 1}

models have benefited greatly from this stream of research. Table 1.1 gives an overview of the richness of the option pricing volatility models universe created by academic research.

This literature has yielded important results concerning the specifications required for properly pricing options. It appears important that a continuous time (discrete time) volatility model includes stochastic volatility (time-varying volatility) to capture the smile. But it is even more crucial to include correlation between return and volatility as well as jumps (nonnormal innovations) to capture the volatility skew.

The same literature has also provided evidence on the non-model related routine to adopt in order to improve models pricing performance in general. It has been demonstrated (Duan, 1995) that directly calibrating models on options prices (under Q) rather than on the underlying (under P) provides much better pricing performances due to the discrepancy between the risk-neutral and the physical density. Additionally, although theoretically inconsistent, continuous recalibration (re-calibrating models every period on the daily crosssection of option) also drastically improves pricing performances of virtually all models (Christoffersen and Jacobs , 2004a).

This last discovery highlights an important shift in the approach to studying option markets. The initial intention was to explain option prices and the later intention was to learn from option prices. Indeed, the bulk of research is now dedicated to option implied information. Pricing options and extracting option implied information are two sides of the same coin, but have different implications. Parameters calibrated on option prices are option implied parameters that use cross-sections of options as a source of information. This is in contrast with the studies that estimate models on the time series of the underlying. Calibrating parameters under Q, directly on options prices, has become common practice. The risk-neutral parameters are the relevant parameters for pricing options. Empirical studies have demonstrated that this leads to an improved pricing performance. This procedure also permits an alignment of the loss function used for the optimization with the end use objective. Indeed an empirical option pricer is not interested in minimizing the likelihood function of a model on the underlying distribution but is interested in minimizing the difference between market option prices and predicted prices.

\subsection{LIMITATIONS AND THESIS CONTRIBUTIONS}

However, the framework for directly calibrating and comparing models on options prices is less developed than the usual time series maximum likelihood estimations. This lack of framework leads scholars and practitioners to rely on rules of thumb rather than on theoretically motivated or evidence-based choices. The next two chapters of this thesis provide an objective assessment of the benefits and limitations of alternative approaches regarding model performance comparison and data selection. Building upon these findings, 
we propose a novel framework to circumvent the inherent limitations of the currently available approaches.

\section{Second chapter}

In the second chapter, we identify a common flaw in the approach to evaluating option pricing model performance. We demonstrate that a fair approach to comparing alternative models' performance needs to account for uncertainty in reported pricing performance.Empirically, in the option pricing literature, researchers adopt particular loss functions ignoring measurement, model and parameter uncertainty. Pricing error is the result of a difference between observed prices and predicted prices, where the predicted prices are dependent on a particular model and the values assigned to the parameters of this model. The parameters are estimated relying on the choice of a loss function and a set of observations. There is estimation uncertainty around these parameter point estimates. This uncertainty is conveyed to the predicted prices and therefore the loss function.

Building on the option pricing loss function bootstrapping technique of Bams, Lehnert and Wolff (2009), we provide a framework for constructing a confidence interval around the loss function. This confidence interval can be interpreted like a classical parameter confidence interval. This additional information allows us to affirm that the true performance of a model lies between two limits with a certain probability. This approach contrasts with the available techniques that rely solely on the absolute value of the loss function. Furthermore, this approach allows for a formal specification test to compare alternative models or approaches.

In an empirical application of our framework, with a discrete time asymmetric GARCH model on S\&P 500 options, we show that there is significant uncertainty around the traditionally reported absolute loss function. Furthermore we find short-term persistence but long term heterogeneity in cross-sectional loss function distribution. The shape of the distribution of the loss function does not change significantly for a relatively close crosssection of options. This is in contrast with the strongly diverging pattern of the loss function distribution across a longer time horizon. The relative merit of a model is therefore time dependent. We interpret this as additional evidence in favour of continual recalibration carrying out the option pricing exercise at the cross-section level. This finding also reinforces the premise that performance evaluation should be carried out at the period level. We also confirm the evidence that parsimonious volatility models are sufficient for pricing options (Christoffersen and Jacobs, 2004a). We can demonstrate that the pricing surface is flat by investigating the bootstrapped variance-covariance matrix. 


\section{CHAPTER 1}

\section{Third chapter}

The third chapter of the thesis investigates the other aspect of the empirical option pricing exercise, namely model estimation. The resulting parameter estimates are heavily influenced by the estimation background. For example, the role of the loss function on parameter estimates has been highlighted by Christoffersen and Jacobs (2004b) and Bams et al. (2009). But the implications of the data selection process on parameter estimates remain largely unexplored. In this section of the thesis, we provide a framework to quantify the effects of data selection on pricing performance, and we investigate the causes of these substantial effects.

What data should be used to calibrate a model? This is a relevant question since the parameters reflect the characteristics of the data information content. It is common practice to carefully select and clean the data that will be used for that purpose. However, we observe that empirical option pricers rely on different filtering procedures to clean the option data. Accordingly, they obtain different samples for estimating the model parameters. We evaluate the impact of the choice of a filtering procedure on the parameter estimates and predicted prices using three well-known filtering procedures found in the literature.

Our empirical design shows that the filtering procedure matters. The same model estimated on these different samples results in alternative sets of parameters. The striking result is that the same model provides different out-of-sample predictions for the same option, depending on the filter used. The sample selection can be thought of as an implicit part of the model specification. Moreover, the lack of consensus on the right exclusion filter to use causes alternative studies to be based on highly divergent samples. This paper provides a methodology to benchmark alternative exclusion filtering rules and to measure the effects of relying on one filter rather than another. We show that there is no single best filter. Every filter is specialized in accurately predicting prices of options with certain characteristics. The ability to properly fit an option price depends on whether the filter includes options with the same characteristics. We show that special consideration should be given to two option characteristics: the maturity and the Put/Call contract type. These two characteristics cause the filter pricing specialization.

The second part of the thesis turns toward more economic questions. We investigate what can be learnt from option markets and how option implied information can be used. An option price depends on the option payoff over a certain time in the future. Accordingly, option prices reflect the investor's forward looking expectations of the underlying distribution. For example, according to CBOE market statistics, the average option premium in 2008 was $\$ 812$. By comparison, in 2012 , this figure was only $\$ 517$. The difference is explained by the higher investor volatility expectations in 2008 , at the heart of the financial crisis, compared to 2012. 
However, it is critical to remember that option information is in fact option prices. This notion of price has important implications since it embeds investor preferences. Investors are eager to pay a high premium for an option that pays off during a state of the world when they have a strong preference for receiving money. This can be the situation even if the probability of this state of the world occurring is low. This is the case for deep out-of-the-money index options. Therefore, option prices are not perfectly linked to the true probability. The probability or the information implied from options is adjusted for risk or for preferences. These risk-adjusted preferences are called 'risk-neutral probability'.

\section{Fourth chapter}

In the fourth chapter, using the variety and richness of the option markets, we bridge the gap between two important research streams in the financial economics literature. On the one hand, it has been shown that uncertainty, independently from risk, has a deep impact on stock markets (Anderson, Ghysels and Juergens, 2009; Bekaert, Engstrom and Xing, 2009). On the other hand, many studies have evaluated the effect of alternative asset (such as oil) price changes on stocks (Driesprong, Jacobsen and Maat, 2008; Kilian and Park, 2009; Baur and McDermott, 2010). To our knowledge, we are the first to evaluate and to compare the impact of oil and gold price uncertainty with S\&P 500 uncertainty on the cross-section of stocks expected returns.

Measuring uncertainty has been proven to be difficult. Using volatility or conditional volatility is an inaccurate uncertainty measure, and relying on analyst forecast disagreement suffers from too many limitations (Anderson et al., 2005). On the contrary, we use the very recently proposed volatility risk premium as a measure of uncertainty (Carr and $\mathrm{Wu}, 2009$; Bali and Zhou, 2013; Buraschi, Trojani and Vedolin, 2014). The volatility risk premium is the difference between the expected physical volatility and the expected volatility implied from options. The option implied volatility reflects investor preferences and expectations. When the distribution of future returns is uncertain, investors bid up option prices and option implied volatilities relative to the true level of expected volatility. This approach provides comparable measures of uncertainty for any asset on which options are written. Using option data from the Commodity Research Bureau, we measure the S\&P 500, oil and gold price uncertainty.

First, we find that all uncertainties globally affect equities in a negative manner. An increase in uncertainty is associated with falling equity prices. The uncertainty computed from S\&P 500 options has a dominant effect. Secondly, we investigate whether uncertainty is a priced factor in the cross-section of expected return, as suggested by Anderson et al. (2009) and Bali and Zhou (2013). The results show that S\&P 500 uncertainty is priced in the cross-section of stock returns. Stocks more exposed to S\&P 500 uncertainty have higher returns. However, we cannot find a significant compensation for oil or gold uncertainty. This result is robust across firm size deciles and business cycles. We interpret this result as evidence that only the macro- 


\section{CHAPTER 1}

economic systematic part of uncertainty is priced in the entire stock universe. However, we show that oil uncertainty is an industry specific priced factor. This finding reflects the results of Pollet (2005) showing that market segmentation at industry level caused different stocks to react differently to oil news.

\section{Fifth chapter}

The fourth chapter of the thesis exploits the discrepancy between physical expectation and risk neutral expectation. The fifth chapter analyses the impact of this difference in a risk management context. Both researchers and practitioners have long been interested in comparing the predictive performance of alternative volatility forecasts. Since Chiras and Manaster (1978) and Beckers (1981), a horse-race has been going on between time series volatility model forecasts and option implied volatility forecasts. The latter forecast has the advantage of being forward looking and more responsive to macro-economic news. However, the implied volatility used to forecast the future physical volatility is a risk neutral measure. With the exception of Prokopczuk and Wese Simen (2014), the entire literature ignores this fact. Many studies conclude that implied volatility is nevertheless a better volatility predictor than time series volatility models.

Although risk management is often a justification for the volatility prediction exercise, virtually no paper evaluates the benefits of using implied volatility in a Value at Risk context. In chapter five, we evaluate the merits of implied volatility for quantile forecast rather than for volatility forecast. We benchmark and compare time series model VaRs with implied volatility VaRs for three equity indices over 23 years of daily data. Also, we formally account for and correct the implied volatilities for the volatility risk premium.

The results of our different tests consistently point in the same direction. Unlike volatility prediction, the implied volatility based VaRs are not able to outperform the time series volatility model based VaRs. This result shows that predicting volatility and predicting a certain quantile of a distribution are two different objectives. We demonstrate that the volatility risk premium severely impairs the VaR predictions based on option implied volatilities. The VaRs based on implied volatilities are too conservative and are upwardly biased. This empirical fact extends the need to adjust for the volatility risk premium. However, neither adjustment used, non-parameteric or parametric, allows option implied volatility VaRs to outperform a Garch model with leverage. The volatility risk premium dynamics need to be modeled with more complexity for quantile prediction than for volatility prediction. Option implied information is of great value but one should bear in mind that this information extracted from options is risk adjusted (under Q) and is therefore not an unbiased estimate of the physical market realization (under P). 


\section{Chapter 2}

\section{Evaluating Option Pricing Model Performance Using Model Uncertainty ${ }^{1}$}

\subsection{INTRODUCTION}

Explaining option prices has been at the center of financial research interest since Black and Scholes (1973). Two complementary streams of research have emerged. The first is dedicated to theoretically developed models that reflect complex data generating processes of the volatility dynamics. The second, more pragmatic, is devoted to assessing, evaluating and comparing the performance of these alternative models empirically.

Our focus is on the latter. In this chapter we suggest a method for deriving the probability distribution of a loss function. This allows for the statistical comparison of the ability of alternative models to price a single cross-section of option prices, either in-sample or out-ofsample. Unlike existing methods, the proposed test does not rely upon a time series of crosssections, nor is it limited to the comparison of absolute pricing errors alone.

How best to measure option pricing model performance depends on the user's perspective. For instance, Bakshi, Cao and Chen (1997) provide three performance criteria, alternatively using internal consistency, pricing error and hedging error as objective evaluation criteria. Pricing error has become the leading criterion across a wide range of studies. The gap between observed and predicted options prices forms the basis of loss function based measures. This has the merit of an intuitive economic interpretation of a model's ability to match observed data. Moreover, this criterion allows for an out-of-sample comparison of models that are very different in nature. The existing option pricing literature provides some critical insights into which model specifications and which estimation and evaluation criteria to use. Hardle and Hafner (2000) and Heston and Nandi (2000) show that adding a leverage effect to the standard autoregressive volatility model leads to a significant reduction of the option pricing error. Furthermore, Christoffersen and Jacobs (2004a) provide empirical evidence that the use of a specification which is richer than a standard asymmetric GARCH model does not provide any further pricing performance improvement. Nonetheless, Barone-

\footnotetext{
${ }^{1}$ This chapter is co-authored with Dennis Bams (Maastricht University) and Thorsten Lehnert (Université du Luxembour)
} 
Adesi, Engle and Mancini (2008) establish that additional pricing error reduction can be achieved by allowing for non-normality specifications of the error term.

Aside from model selection, major developments have occurred on the methodological aspects of parameter estimation of option pricing models. Volatility dynamics which are inferred from information in the underlying time series returns differ from the volatility dynamics that result from a direct calibration on options prices (Engle and Mustafa, 1992). While initially, studies relied on parameter estimates under the physical measure (Bollerslev and Mikkelsen, 1999), it subsequently turned out that parameters estimated under the risk neutral measure explain empirical features of option prices better (Duan, 1996), and significantly reduce the value of the loss function (Christoffersen and Jacobs, 2004a). Accordingly, estimating parameters directly on options prices has become the standard practice (Lehnert, 2003; Barone-Adesi, Engle and Mancini, 2008; and Frijns, Lehnert and Zwinkels, 2010).

While practitioners prefer to recalibrate their model continuously on daily data, academics have suggested calibrating the model only once (Hull and Suo, 2002). Although less theoretically based, the practitioners' approach has proven to deliver better pricing performance (Christoffersen and Jacobs, 2004a), leading to general adoption of this technique in academia as well.

Despite the important insights in the existing literature regarding option pricing performance, we feel that there is scope for improvement in the performance evaluation criterion, which is usually limited to a comparison of point estimates of loss functions. Our proposed performance evaluation criterion explicitly incorporates the effect of measurement, model and parameter uncertainty. Following the bootstrapping approach in Bams, Lehnert and Wolff (2009), we adopt an entire probability distribution function for the loss function, which facilitates a formal specification test to compare alternative models and approaches.

The absence of analytical solutions for the distribution of the loss function is a cause for the lack of statistical tests in option pricing. Bootstrapping techniques offer a framework to provide such tests. This is a data driven method that evaluates, even in the ideal situation of a known data generating process, whether data imperfections allows us to precisely estimate and evaluate the option pricing model.

We fit and bootstrap option prices within a cross-section of options of a given day. This approach provides a loss function distribution on daily basis which accounts for the time varying quality of data used for continuously re-estimated models. Rather than a naïve bootstrap we provide a specific cross-sectional block bootstrapping design to acknowledge for the residual dependency across moneyness and maturity. 
Since the literature advocates a continuous recalibration, this extends the need to compare model performance to a cross-section by cross-section level. Our empirical results confirm the fundamental difference in nature between alternative cross-sections of options. This heterogeneity in cross-sections of option prices over time makes statistical tests as proposed by Diebold and Mariano (1995) and applied for option pricing purposes in Christoffersen and Jacobs (2004a) less useful. This is a further argument for employing statistical inference at a single cross-section, as proposed in our testing framework.

In the following section, we introduce our statistical framework and provide an empirical application of the framework, with a discrete time asymmetric GARCH model on S\&P 500 options.

\subsection{THE ECONOMETRIC FRAMEWORK}

The statistical testing framework for measuring option pricing model performance is general in the sense that it can be applied to any class of models such as continuous time models, discrete time models or ad hoc models. We present the framework in the context of a specific discrete time asymmetric GARCH volatility specification, to allow for realistic empirical findings and implications.

The choice of the discrete time asymmetric GARCH volatility specification is motivated by the evidence in the literature that such a model describes option pricing data features relatively well. Within the discrete time volatility model class, Christoffersen and Jacobs (2004a) show that in order to obtain good pricing performance, clustering and leverage are two important effects to account for. In addition to clustering and leverage, other features have been proposed in the literature, but none of them have turned out to be very effective for additional pricing performance. Hsieh and Ritchken (2005) show that GARCH models are capable of explaining a significant portion of the volatility smile. Lehar, Scheicher, and Schittenkoph (2002) demonstrate the relative outperformance of GARCH models compared to stochastic volatility models in term of out-of-sample options pricing performance. We use the volatility model of Frijns, Lehnert and Zwinkels (2010). This discrete-time specification is one of the numerous available asymmetric GARCH models, which features both volatility clustering and volatility leverage.

Discrete-time volatility models including the estimation of the long term unconditional volatility parameter are known to be unstable. Therefore, we have chosen to approximate the long term unconditional volatility in the model with the realized long term volatility. This approach is similar to variance targeting, and has been proven to stabilize the volatility process at no pricing performance costs (Bams, Lehnert, and Wolff, 2009).

The continuously compounded returns of the underlying asset follow the traditional process: 


\section{CHAPTER 2}

$r_{t}=\mu+\sigma_{\mathrm{t}} \varepsilon_{\mathrm{t}}$

$\varepsilon_{\mathrm{t}} \sim N(0,1)$

The volatility dynamics are defined as follows:

$\operatorname{Ln}\left(\sigma_{\mathrm{t}+1}^{2}\right)=\operatorname{Ln}\left(\sigma_{\mathrm{t}}^{2}\right)+\frac{1}{2} \alpha\left(\sigma_{\mathrm{t}}^{2}-\overline{\sigma_{\mathrm{t}}^{2}}\right)+\frac{1}{2}\left\{\beta_{0} \max \left\{\varepsilon_{\mathrm{t}}, 0\right\}-\beta_{1} \min \left\{\varepsilon_{\mathrm{t}}, 0\right\}\right\}$

Volatility is a function of two equally weighted components. The first component, $\left\{\alpha\left(\sigma_{\mathrm{t}}^{2}-\right.\right.$ $\left.\left.\overline{\sigma_{\mathrm{t}}^{2}}\right)\right\}$, drives the mean reversion of volatility where $\alpha$ is the parameter that determines the speed of mean-reversion and $\overline{\sigma_{t}^{2}}$ is the long-term unconditional volatility. The second component, $\left\{\beta_{0} \max \left\{\varepsilon_{\mathrm{t}}, 0\right\}-\beta_{1} \min \left\{\varepsilon_{\mathrm{t}}, 0\right\}\right\}$, features the leverage effect where $\beta_{0}$ and $\beta_{1}$ allow for an asymmetric response to positive and negative shocks, respectively.

Our pricing performance results are in line with Barone-Adesi, Engle and Mancini (2008) who use a similar sample and another asymmetric GARCH specification. This provides reassurance that the model used is representative of a wider class of asymmetric GARCH-type model.

Following Duan (1995), we apply the Local Risk Neutral Valuation Relationship to arrive at the return dynamics under the risk adjusted probability measure. The Local Risk Neutral Valuation Relationship specifies that the one period forward conditional variance is the same under both the actual and risk adjusted dynamics. The conditional expectation of the underlying under the risk neutral probability holds that:

$E^{Q}\left[\exp \left(r_{t}\right) \mid \Omega_{\mathrm{t}-1}\right]=\exp \left(r_{t}^{f}\right)$

where $r_{t}^{f}$ is the risk free rate at time t. This results in the following risk adjusted process for the return dynamics:

$r_{t}=r_{t}^{f}-\frac{1}{2} \sigma_{\mathrm{t}}^{2}+\sigma_{\mathrm{t}} \varepsilon_{\mathrm{t}}$

$\varepsilon_{t} \mid \Omega_{\mathrm{t}-1} \sim N(0,1)$

The volatility dynamics, as given in equation (2.3), remain unchanged when transitioning from actual to risk-adjusted return dynamics.

From the Principle of Risk Neutral Valuation, it follows that option prices are determined as the expected option payoff function discounted with the risk free rate, where expectations are taken under the risk adjusted probability measure, Q:

$C_{i t}=\exp \left(-r^{f}\left(t, T_{i t}^{C}\right) \times\left(T_{i t}^{C}-t\right)\right) \times E^{Q}\left\{\max \left[S\left(T_{i t}^{C}\right)-K_{i t}^{C}, 0\right] \mid \Omega_{\mathrm{t}-1}\right\}$ 
$P_{i t}=\exp \left(-r^{f}\left(t, T_{i t}^{P}\right) \times\left(T_{i t}^{P}-t\right)\right) \times E^{Q}\left\{\max \left[K_{i t}^{P}-S\left(T_{i t}^{P}\right), 0\right] \mid \Omega_{\mathrm{t}-1}\right\}$

Where $C_{i t}$ and $P_{i t}$ are, respectively, the call $i$ and put $i$ prices at time $t ; T_{i t}^{C}$ and $T_{i t}^{P}$ are the associated times-to-maturity of call $i$ and put $i$ at time $t ; K_{i t}^{C}$ and $K_{i t}^{P}$ are the strike prices of the call and put options $i$ at time $t$. With $r^{f}(t, T)$ we indicate the risk free rate at time t, appropriately reflecting the term structure of interest rates for the remaining time-to-maturity, $(T-t)$. Finally, $S(T)$ is the value of the underlying stock at time $T$.

In the absence of closed or semi-closed form solutions, the option payoff distribution in equations (2.7) and (2.8) is obtained through Monte Carlo Simulation. Following Duan and Simonato (1998), we use the Empirical Martingale Simulation (EMS) approach to reduce the required number of simulations for convergence.

Subsequently, cross-sectional parameter estimation follows by the choice and subsequent minimization of a loss function that calibrates modeled option prices to observed option prices. The proposed statistical framework is general in the sense that it works for alternative loss functions. Following the recommendation of Bams, Lehnert, Wolff (2009), for the empirical application, the root mean squared error (RMSE) of absolute pricing errors is chosen as loss function. Parameter estimation for cross-section $t$, follow from minimization of the following loss function:

$R M S E_{t}=\sqrt{\frac{1}{N_{t}^{C}+N_{t}^{P}}\left(\sum_{i=1}^{N_{t}^{P}}\left(P_{i t}-\hat{P}_{i t}\right)^{2}+\sum_{i=1}^{N_{t}^{C}}\left(C_{i t}-\hat{C}_{i t}\right)^{2}\right)}$

where $N_{t}^{C}$ and $N_{t}^{P}$ are the respective number of call and put options in cross-section $t ; \hat{C}_{i t}$ and $\hat{P}_{i t}$ are the model call $i$ and put $i$ prices at $t$, following from equations (2.5) to (2.8); $C_{i t}$ and $P_{i t}$ are the observed call $i$ and put $i$ options prices at time $t$. The loss function in equation (2.9) is minimized for each cross-section of option prices $t$ separately using the Newton-Raphson algorithm, resulting in a separate set of parameters estimates $\left(\widehat{\alpha}, \widehat{\beta_{0}}, \widehat{\beta_{1}}\right)_{t}$ as well as an accompanying value for the loss function, $\widehat{R M S E}_{t}$ for each cross-section $t$.

The absence of an analytical or easily derivable distribution function for the loss function results in a lack of statistical inference in the bulk of the option pricing performance evaluation literature. Instead, comparison of absolute loss functions for model selection characterizes the option pricing model literature. Bakshi, Cao and Chen (1997) benchmark the alternative continuous time models based on the absolute RMSE; Heston and Nandi (2000) use the RMSE accompanied by other absolute loss functions to demonstrate the outperformance of their model over available alternative specifications; Barone-Adesi, Engle, and Mancini (2008) use a battery of absolute loss functions to depict the pricing improvement 
of a model that includes historical innovations compared to Gaussian or other parametric alternatives.

Christoffersen and Jacobs (2004a), provide more statistical validity for option pricing model comparison using the Diebold-Mariano (DM) test. This test is virtually a z-test on a time series differential between two forecasts of losses corrected for serial correlation. In option pricing, the loss function differential time series is obtained by iterative cross-section by cross-section estimation and loss function evaluation pooled together over all cross-sections. The DM test is designed for out-of-sample forecast comparison, which excludes in-sample testing. Moreover, the DM test is not intended for model selection (Diebold, 2012). In our opinion, the principal limitation of the DM test resides in its inability to address the model performance for a single cross-section.

The time series requirement, pooling the information in many cross-sections, would at best be an approximation for the model performance at a single cross-section. This would require homogeneity of the information in the alternative cross-sections. In fact, there are two sources of heterogeneity, as we will also demonstrate in the empirical application. First, model performance is affected by change in the economic environment. Diebold and Mariano (1995) discuss the effect of business cycles on relative predictability. Second, the iterative recalibration comes at a cost. The data contained in each cross-section is evolving through time. The total number of observations and the qualitative composition of cross-sections vary significantly. This suggests that over time, the informational content of alternative crosssections is changing and is therefore different.

To meet the need for a cross-sectional test, we propose a bootstrap based methodology to estimate the distribution of each cross-section RMSE separately. In the general finance literature, the use of bootstrap to overcome the lack of analytical solutions is a common practice (Bams, Lehnert and Wolff, 2005; Ledoit and Wolf, 2008; Hansen, Lunde and Nason, 2011). However, the use of bootstrapping in the specific field of option pricing has been more limited. Christoffersen and Jacobs (2004b) use a jackknife approach to study the pricing effect of alternative loss functions on a contemporaneous out-of-sample observation. Bams, Lehnert and Wolff (2009) bootstrap and summarize the loss function distribution into a statistic to assess loss function selection accounting for uncertainty. Finally, in a simulation and application study, Yatchew and Härdle (2006) show in the context of nonparametric state price density estimation that relying on a wild bootstrap to construct call function confidence intervals leads to reasonable results. We take this evidence as a good indication that bootstrapping option pricing errors is an appropriate method of depicting pricing uncertainty.

Bootstrapping is a re-sampling technique to obtain the distribution of a particular statistic. This method is applied below to obtain the probability distribution of the loss function for a single cross-section. The initial estimation step produces parameter estimates, fitted option 
prices, residuals and accompanying value for the loss function. The difference between the observed market price and theoretical price is the residual. We introduce the following matrix notation:

$$
\begin{array}{rlrl}
C_{t} & \equiv\left(C_{i t}, \ldots, C_{N_{t}^{C}, t}\right)^{\prime} & t=1, \ldots, T \\
P_{t} \equiv\left(P_{i t}, \ldots, P_{N_{t}^{P}, t}\right)^{\prime} & t=1, \ldots, T
\end{array}
$$

where $C_{t}$ and $P_{t}$ are two vectors respectively $\left(N_{t}^{C} \mathrm{X} 1\right)$ and $\left(N_{t}^{P} \mathrm{X} 1\right)$ of the observed call and put market prices; $\hat{C}_{t}$ and $\hat{P}_{t}$ are two vectors respectively $\left(N_{t}^{C} \mathrm{X} 1\right)$ and $\left(N_{t}^{P} \mathrm{X} 1\right)$ of the modeled call and put prices:

$$
\begin{array}{ll}
\hat{C}_{t} \equiv\left(\hat{C}_{i t}, \ldots, \hat{C}_{N_{t}^{C}, t}\right)^{\prime} & t=1, \ldots, T \\
\hat{P}_{t} \equiv\left(\hat{P}_{i t}, \ldots, \hat{P}_{N_{t}^{P}, t}\right)^{\prime} & t=1, \ldots, T
\end{array}
$$

We introduce two vectors of residuals:

$$
\begin{array}{ll}
\eta_{t}^{C} \equiv\left(\eta_{i t}^{C}, \ldots, \eta_{N_{t}^{C}, t}^{C}\right)^{\prime} & t=1, \ldots, T \\
\eta_{t}^{P} \equiv\left(\eta_{i t}^{P}, \ldots, \eta_{N_{t}^{P}, t}^{P}\right)^{\prime} & t=1, \ldots, T
\end{array}
$$

where it holds that:

$$
\begin{array}{lll}
\eta_{i t}^{C}=C_{i t}-\hat{C}_{i t} & i=1, \ldots, N_{t}^{C} & t=1, \ldots, T \\
\eta_{i t}^{P}=P_{i t}-\hat{P}_{i t} & i=1, \ldots, N_{t}^{P} & t=1, \ldots, T
\end{array}
$$

It is possible to create a bootstrapped sample by constructing "bootstrapped market prices" for each observations drawing residual with replacement. $C_{t}^{*}$ and $P_{t}^{*}$ are two vectors respectively $\left(N_{t}^{C} \mathrm{X} 1\right)$ and $\left(N_{t}^{P} \mathrm{X} 1\right)$ of the bootstrapped call and put prices, defined as:

$$
\begin{array}{rlrl}
C_{t}^{*} & \equiv\left(C_{i t}^{*}, \ldots, C_{N_{t}^{C}, t}^{*}\right)^{\prime} & t=1, \ldots, T \\
P_{t}^{*} \equiv\left(P_{i t}^{*}, \ldots, P_{N_{t}^{P}, t}^{*}\right)^{\prime} & t=1, \ldots, T
\end{array}
$$

where $B_{t}^{C}$ and $B_{t}^{P}$ are two vectors respectively $\left(N_{t}^{C} \mathrm{X} 1\right)$ and $\left(N_{t}^{P} \mathrm{X} 1\right)$ obtained from drawing with replacement $N_{t}^{C}$ and $N_{t}^{P}$ observations from the $\eta_{i t}^{C}$ and $\eta_{i t}^{P}$ vectors, defined as:

$$
B_{t}^{C}=\left(B_{i t}^{C}, \ldots, B_{N_{t}^{C}, t}^{C}\right)^{\prime} \quad t=1, \ldots, T
$$




$$
B_{t}^{P}=\left(B_{i t}^{P}, \ldots, B_{N_{t}^{P}, t}^{P}\right)^{\prime} \quad t=1, \ldots, T
$$

It holds that:

$$
\begin{array}{ll}
C_{t}^{*}=\hat{C}_{t}+B_{t}^{C} & t=1, \ldots, T \\
P_{t}^{*}=\hat{P}_{t}+B_{t}^{P} & t=1, \ldots, T
\end{array}
$$

The drawing procedure to obtain equation (2.20) and (2.21) can be replicated independently $\mathrm{S}$ times to obtain $\mathrm{S}$ bootstrapped samples. The optimization of equation (2.9) is performed on each of the $\mathrm{S}$ bootstrapped samples for cross-section $t$. This mechanism procures the desired distribution reflecting estimation uncertainty of the estimated parameters $\left(\hat{\alpha}, \hat{\beta}_{0}, \hat{\beta}_{1}\right)_{t}^{(1)}, \ldots,\left(\hat{\alpha}, \hat{\beta}_{0}, \hat{\beta}_{1}\right)_{t}^{(S)}$ and loss functions $\widehat{R M S E}_{t}^{(1)}, \ldots, \widehat{R M S} E_{t}^{(S)}$.

We acknowledge that the asymptotic consistency of the bootstrapping procedure described is not systematically insured. Nevertheless, in absence of reasonable simulation setting, we show that the first moment of the bootstrapped loss functions distributions match closely the actual loss function at any point in time. We interpret this as an encouraging sign that the bootstrapped distribution converge to the actual distribution.

The distribution of the residuals in different maturity and moneyness categories is strongly divergent. The deep in-the-money long maturity residuals are in absolute (relative) terms larger (smaller) and more (less) volatile than deep out-of-the-money short maturity residuals. To account for this cross-sectional heterogeneity across moneyness and maturity, a block bootstrapping technique is pursued as applied in Bams, Lehnert and Wolff (2009). Bootstrapped prices are formed by drawing residuals from the block matching the moneyness/maturity block of the particular option. For that purpose, similar to Barone-Adesi, Engle, and Mancini (2008), 12 blocks are formed with respect to maturity $(60>T, 60 \leq T \leq$ $160, T>160)$ and moneyness $(0.85>M, 0.85 \leq M \leq 1,1<M<1.15, M>1.15)$. To have a sufficiently large historical sample, we bootstrap residuals from the contemporaneous and the 3 previous cross-sections. This procedure avoids a downward bias in the bootstrapped confidence interval due to relying too often on the same bootstrapped error terms, while short term homogeneity in option prices cross-sections safeguards the appropriateness of drawing from this larger sample.

\subsection{DATA}

We use European S\&P 500 index options (SPX). The SPX option market is the most active in the world, making it popular in the option pricing literature (Barone-Adesi, Engle, and Mancini, 2008), and this allows for a comparison with findings in existing literature. The closing prices of each Wednesday are used. These data are collected from OptionMetrics and cover 3 years of data from January 2002 to December 2004, including 155 Wednesdays. 
Filtering criteria are comparable to Barone-Adesi, Engle, and Mancini (2008). Only out-ofthe-money options are selected. Options with a maturity lower than or equal to 10 days and higher than or equal to 360 days are filtered-out. Similarly, options with an implied volatility higher than $70 \%$ and options with a price lower than or equal to $\$ 0.05$ are excluded. In addition to these conventional rules, if in the same cross-section two options have the same maturity and strike price, only the most traded option remains in the sample. The S\&P 500 dividend yields and zero-coupon default free interest rates are also collected from OptionMetrics.

Table 2.1 presents the average price, average implied volatility and number of contracts per category, where a category is defined by moneyness and maturity. We observe the wellknown characteristics of the volatility smile, that deep out-of-the-money puts and calls exhibit a higher implied volatility than close to the money put and call options. This difference in implied volatility decreases with maturity.

Table 2.1: Average price, BS-Implied volatility and number of contract by moneyness and maturity

\begin{tabular}{|c|c|c|c|c|c|}
\hline & & & \multicolumn{3}{|c|}{ Maturity } \\
\hline & & & $<60$ & 60 to 160 & $>60$ \\
\hline \multirow{12}{*}{$\begin{array}{l}\tilde{E} \\
\stackrel{0}{0} \\
\stackrel{0}{0} \\
\sum\end{array}$} & \multirow{3}{*}{$<0.85$} & Price & 0.769 & 2.534 & 8.597 \\
\hline & & $\sigma_{B S}$ & 0.321 & 0.277 & 0.237 \\
\hline & & $\mathrm{Nb}$ Contract & 1766 & 2342 & 2800 \\
\hline & \multirow{3}{*}{$0.85-1$} & Price & 8.389 & 19.063 & 38.584 \\
\hline & & $\sigma_{B S}$ & 0.185 & 0.182 & 0.179 \\
\hline & & $\mathrm{Nb}$ Contract & 3341 & 2123 & 2273 \\
\hline & \multirow{3}{*}{$>1$ to $<1.15$} & Price & 7.431 & 15.724 & 34.761 \\
\hline & & $\sigma_{B S}$ & 0.145 & 0.142 & 0.143 \\
\hline & & $\mathrm{Nb}$ Contract & 2929 & 2111 & 2211 \\
\hline & \multirow{3}{*}{$>1.5$} & Price & 0.339 & 0.840 & 3.900 \\
\hline & & $\sigma_{B S}$ & 0.286 & 0.201 & 0.154 \\
\hline & & $\mathrm{Nb}$ Contract & 1571 & 2249 & 3048 \\
\hline
\end{tabular}

Notes: The option sample characteristic averages are summarized by maturity and moneyness. Moneyness in this table is computed as $(\mathrm{k} / \mathrm{s})$. Since only out of the money options are conserved, all options with a moneyness lower than 1 are put options. All options with a moneyness higher than 1 are call options. Maturities are in days. $\sigma_{B S}$ refers to the Black and Scholes implied volatility.

The division of options within a moneyness/maturity category is balanced with respect to the proportion of puts and calls. Put (call) options represent $51 \%$ (49\%) of the sample; $48 \%$ of the options are deep out-of-the-money with a strike/underlying ratio under 0.85 for puts and over 1.15 for calls; $52 \%$ of the options are less out-of-the-money with a strike/underlying ratio over or equal to 0.85 for puts and under or equal to 1.15 for calls. Long, medium and short maturity options represent respectively $36 \%, 31 \%$ and $33 \%$ of the sample. 


\section{CHAPTER 2}

To assess the sources and effects of cross-sectional heterogeneity, we collect descriptive statistics of market conditions and sample conditions at the individual cross-sectional level. Table 2.2, Panel A reports the average, standard deviation, minimum and maximum values for market conditions and cross-sectional composition variables. Cross-sections differ strongly with respect to both types of variables.

Table 2.2: Sample composition and market conditions descriptive statistics

Panel A

\begin{tabular}{cllll}
\hline & Mean & Std. Dev. & Min & Max \\
\cline { 2 - 5 } Market Conditions & & & & \\
Past Returns & 0.01 & 0.08 & -0.23 & 0.25 \\
Realized Volatility & 0.17 & 0.09 & 0.07 & 0.54 \\
VIX & 0.21 & 0.07 & 0.11 & 0.42 \\
Leverage & 0.27 & 0.07 & 0.15 & 0.47 \\
Local Volatility & 0.17 & 0.06 & 0.08 & 0.36 \\
Sample Compositions & & & & \\
Average Price & 11.53 & 2.03 & 6.80 & 16.78 \\
Average Moneyness & 0.19 & 0.04 & 0.13 & 0.35 \\
Average Maturity & 135.18 & 15.30 & 108.33 & 178.14 \\
Call \% & 0.49 & 0.13 & 0.24 & 0.81 \\
Cheap Option \% & 0.31 & 0.04 & 0.23 & 0.39 \\
\hline
\end{tabular}

Panel B

\begin{tabular}{|c|c|c|c|c|c|c|c|c|c|c|}
\hline & $\begin{array}{c}\text { Past } \\
\text { Returns }\end{array}$ & $\begin{array}{l}\text { Realized } \\
\text { Volatility }\end{array}$ & VIX & Leverage & $\begin{array}{c}\text { Local } \\
\text { Volatility }\end{array}$ & $\begin{array}{l}\text { Average } \\
\text { Price }\end{array}$ & $\begin{array}{c}\text { Average } \\
\text { Moneyness }\end{array}$ & $\begin{array}{l}\text { Average } \\
\text { Maturity }\end{array}$ & Call \% & $\begin{array}{c}\text { Cheap } \\
\text { Option \% }\end{array}$ \\
\hline Past Returns & 1 & & & & & & & & & \\
\hline Realized Volatility & $-0.59 * * *$ & 1 & & & & & & & & \\
\hline VIX & $-0.64 * * *$ & $0.84 * * *$ & 1 & & & & & & & \\
\hline Leverage & 0.07 & $-0.43 * * *$ & $-0.45^{* * * *}$ & 1 & & & & & & \\
\hline Local Volatility & $-0.63 * * *$ & $0.83 * * *$ & $0.99 * * *$ & $-0.46^{* * *}$ & 1 & & & & & \\
\hline Average Price & $-0.42 * * *$ & $0.54 * * *$ & $0.61 * * *$ & $-0.14^{*}$ & $0.62 * * *$ & 1 & & & & \\
\hline $\begin{array}{l}\text { Average } \\
\text { Moneyness }\end{array}$ & $-0.51 * * *$ & $0.75 * * *$ & $0.93 * * *$ & $-0.5 * * *$ & $0.94 * * *$ & $0.55 * * *$ & 1 & & & \\
\hline Average Maturity & 0.04 & 0.1 & 0.07 & -0.01 & 0.09 & $0.57 * * *$ & $0.22 * * *$ & 1 & & \\
\hline Call \% & $-0.71 * * *$ & $0.74 * * *$ & $0.86 * * *$ & $-0.41 * * *$ & $0.86 * * *$ & $0.64 * * *$ & $0.78 * * *$ & 0.11 & 1 & \\
\hline Cheap Option \% & -0.07 & 0.12 & $0.21 * * *$ & $-0.34 * * *$ & $0.21 * * *$ & $-0.42 * * *$ & $0.32 * * *$ & $-0.4 * * *$ & $0.18 * *$ & 1 \\
\hline
\end{tabular}

Notes: Standard errors in parentheses $* * * \mathrm{p}<0.01,{ }^{* *} \mathrm{p}<0.05,{ }^{*} \mathrm{p}<0.1$

The table presents the descriptive statistics of Sample composition and market variables in Panel A. Past return and realized volatility are computed respectively over 3 months and 14 days. Past return and realized volatility are measured for each crosssection. Sample compositions refers to information about each of the 152 (2002 to 2004) samples used. Call \% refers to the percentage of call option in the sample. Cheap Option \% is the percentage of option below $1 \$$. Average moneyness is computed as the absolute value of $(\mathrm{K} / \mathrm{S})-1$. Panel B reports the correlation matrix. 
The selected sample period shows changing market conditions. From 2002 to 2004, the average yearly volatility is $16.6 \%$. Some extremely high volatility regimes are observed at the beginning of the sample, reaching 54.1\%. In both 2003 and 2004, the volatility is much lower; the minimum observed yearly volatility is $7.1 \%$. The high volatility year 2002 coincides with a bearish market. The worst 3 months loss equals $-23 \%$, while in the subsequent year, the market increased by $25 \%$ within 3 months. These statistics highlight the significant change in the underlying physical and risk neutral probability measure across time.

Table 2.2 Panel B reports high correlations between market and sample composition variables. These correlations are mechanically magnified by the exclusion filtering rule used to select the options included in our sample. The sampling condition causing this crosssection composition market conditions dependence is the restriction to include only out-of-the money options. Under this condition, a turbulent market characterised by low return, high realized volatility, and high VIX, results in subsequent cross-sections that are over-dominated by slightly out-of-the-money call options. When a market crashes, the majority of the put options become in-the-money and are automatically excluded from the sample. The reverse occurs for previously in-the-money call options. These newly included call options are concentrated in a close-to-money category, changing the distribution of option moneyness in the cross-section.

The resulting composition shifts are potentially substantial. Call options representation in a cross-section ranges between $24 \%$ and $81 \%$. Hence, the balanced distribution of puts and calls found for the total sample often does not hold up at the single cross-sectional level. The accompanying variation in average moneyness is also meaningful, which is important because options with different levels of moneyness carry different information regarding the risk neutral distribution. Average moneyness per cross-section ranges between 13\% and 35\%. Average option prices per cross-section take a minimum value of $\$ 6.80$ and a maximum value of $\$ 16.78$. The low average options prices are driven by an important concentration of "very cheap options". For some cross-sections, we observe up to almost $40 \%$ of options with prices below $\$ 1$. Absolute prices are relevant, since we use the RMSE as loss function. The composition and distribution of option prices in a particular cross-section have an implicit effect on the weight allocated to different observations and hence affect the estimation results.

\subsection{EMPIRICAL ANALYSIS}

\section{Parameter estimates - cross-section by cross-section}

Table 2.3 presents summary statistics for the cross-section by cross-section estimation results of the volatility specification in equation (2.3), by minimizing the objective function in equation (2.9). The reported numbers are summarizing the individual results from 155 crosssectional parameter estimates. The average in-sample RMSE (\$1.12) is comparable to the 


\section{CHAPTER 2}

Barone-Adesi, Engle, and Mancini (2008) in-sample statistic for the Heston Nandi model. This statistic confirms that our volatility specification has a pricing performance similar to other asymmetric GARCH type models and fits the data sufficiently well. All coefficients display expected values accounting for the clustering and asymmetric dynamics as reflected by the positive $\beta_{1}$ coefficient.

These results pinpoint the heterogeneity over the alternative cross-sections. Loss functions are highly fluctuating over time, ranging between a minimum of $\$ 0.61$ to a maximum of $\$ 2.38$. Different regimes of pricing errors exist. Cross-sections of options relatively mispriced (wellpriced) tend to be followed by other mispriced (well-priced) cross-sections. The observed heterogeneity over time restricts the possibility of pooling many cross-sections to arrive at reliable test statistics, and supports instead our proposed block bootstrapping approach, where only a limited number of cross-sections are pooled for inference purposes.

Table 2.3: Parameter estimates and resulting in-sample RMSE

\begin{tabular}{|c|c|c|c|c|c|}
\hline & $\alpha$ & $\beta_{0}$ & $\beta_{1}$ & $\begin{array}{c}\text { Local } \\
\text { Volatility }\end{array}$ & RMSE \\
\hline Mean & -0.025 & -0.288 & 0.272 & $16.5 \%$ & 1.124 \\
\hline SD & 0.01 & 0.065 & 0.066 & $6.4 \%$ & 0.401 \\
\hline Min & -0.089 & -0.569 & 0.149 & $8.2 \%$ & 0.611 \\
\hline Max & -0.003 & -0.155 & 0.466 & $35.7 \%$ & 2.381 \\
\hline 1st quartile & -0.029 & -0.324 & 0.215 & $11.5 \%$ & 0.832 \\
\hline 3rd quartile & -0.02 & -0.24 & 0.318 & $20.2 \%$ & 1.327 \\
\hline
\end{tabular}

Notes: The present statistics result from the 155 calibrations performed every Wednesday. The statistics of all the parameters estimated and the loss function are displayed. The local volatility is expressed in yearly volatility.

\section{Appropriateness of bootstrapping design}

To get a sense of the appropriateness of our bootstrapping design, Figure 2.1 Panel A plots observed prices against the average of bootstrapped prices. Even with a limited number of repetitions, the mean bootstrapped prices match the observed data well, as illustrated by the plots being concentrated within a tight range along the diagonal line.

Figure 2.1 Panel B compares the time series of cross-sectional RMSE resulting from the nonlinear least squares optimization in (9) and the average of the accompanying bootstrapped RMSEs for each point in time. The average bootstrapped RMSE is closely tracking the estimated RMSE, capturing the changes in economic regime. We interpret these findings as a good indication of the reliability of our bootstrapping procedure. 
We also investigate the properties of the bootstrap by a comparison of average bootstrapped prices and average observed prices at option category level (related to moneyness and maturity). Table 2.4 presents the results of mean tests per category. For all categories, equality of means cannot be rejected.

Figure 2.1: Bootstrapped prices and RMSEs
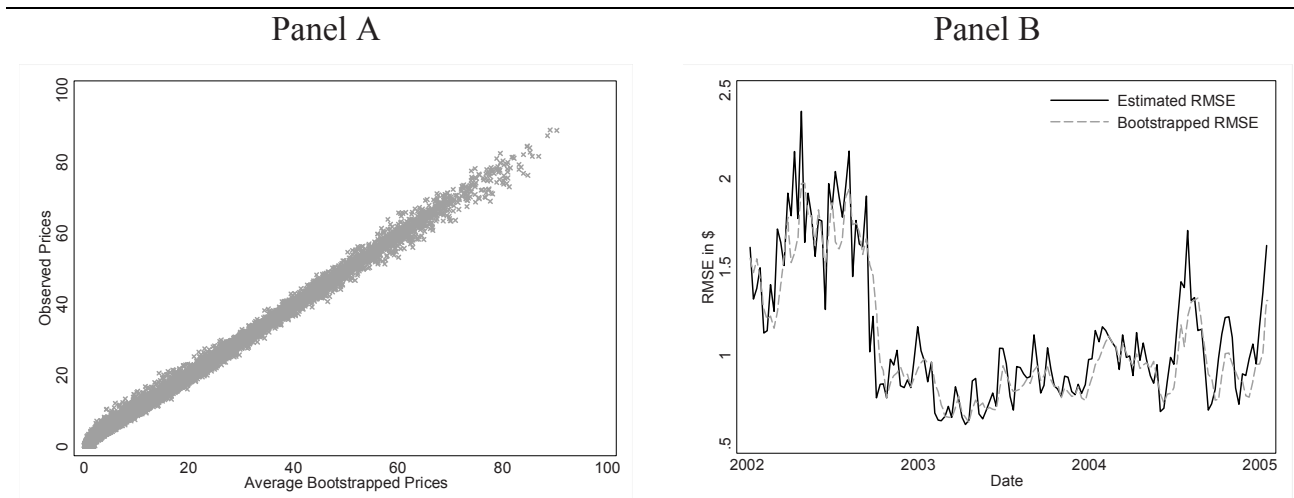

Notes: Panel A plots the actual observed prices against the average of the bootstrapped prices for each observation. Panel B compares the time series of RMSE along different cross-sections. The estimated RMSE is obtained from the original estimation. The bootstrapped RMSE is the mean of the bootstrapped RMSE distribution.

Table 2.4: One sample T-test of the average observed price-average bootstrapped price difference per option category

\begin{tabular}{clll}
\hline & \multicolumn{3}{c}{ Maturity } \\
\cline { 2 - 4 } & $<60$ & 60 to 160 & $>60$ \\
\hline \multirow{2}{*}{0.85} & 0.00 & 0.01 & 0.01 \\
& $(0.56)$ & $(0.67)$ & $(0.16)$ \\
\hline \multirow{2}{*}{$0.85-1$} & -0.01 & -0.00 & -0.01 \\
$\sum_{0}^{0}>1$ to $<1.15$ & $(0.67)$ & $(0.81)$ & $(0.55)$ \\
\cline { 2 - 4 }$>1.5$ & 0.01 & 0.02 & -0.01 \\
& $(0.30)$ & $(0.38)$ & $(0.87)$ \\
\cline { 2 - 4 } & -0.01 & -0.01 & -0.01 \\
& $(0.21)$ & $(0.31)$ & $(0.62)$ \\
\hline
\end{tabular}

Notes: The table presents the average values of the difference between the observed prices and the average bootstrap equivalent prices. In brackets are the P-values associated with a 0 mean difference test.

\section{Parameter inference - standard deviations}

As a result of the cross-sectional estimation and bootstrapping procedure, the distribution and variance-covariance of the parameters estimates are also naturally available at the single cross-sectional level. Dumas, Fleming and Whaley (1998) used as an alternative the time 


\section{CHAPTER 2}

series variations in continuously re-estimated parameters to investigate their dynamics and provide inferences. We compare standard deviations and correlations for parameter estimates both from a time series based approach as in Dumas et al. (1998) and from a cross-sectional approach as resulting from the bootstrap approach. It turns out that bootstrapped parameters statistics are not only available on an individual cross-sectional frequency, but they also contain different information.

Table 2.5 presents the time series based standard deviations as well as the average of crosssectional standard deviations. The time series based standard deviations are notably larger than their cross-sectional counterparts. Results suggest a ratio of 3 to 5 times higher times series based standard deviations. The time series of standard deviations reflects two sources of variations. The first is the variation due to uncertain parameter estimates and over-fitting within each cross-section. The second is the variation across cross-sections driven by a change in economic conditions over time. The cross-sectional approach distinguishes the first source of variation from the second, which makes the standard deviations more reliable as a reflection of parameter uncertainty at the single cross-sectional level.

Table 2.5: Time series and bootstrap parameters information comparison

Panel A: Standard Deviation

\begin{tabular}{lccccc}
\hline & $\alpha$ & $\beta_{0}$ & $\beta_{1}$ & Local Volatility & RMSE \\
\hline SD Time Series & 0.01 & 0.065 & 0.066 & 0.064 & 0.401 \\
SD Bootstrap & 0.003 & 0.017 & 0.015 & 0.002 & 0.074 \\
\hline
\end{tabular}

Notes: The 155 estimations performed result in 155 parameters and loss functions at different point in time. The SD Time series is the standard deviation of these series.

The bootstrap enables a standard deviation for each estimation to be obtained. Therefore 155 SD are obtained. The SD Bootstrap displayed is the average of these 155 SD.

The local volatility parameter, which is the parameter that is used as starting value for the volatility process in equation (2.3), shows a difference that is even more pronounced, with a ratio of 32 . Local volatility tracks economic conditions extremely closely, since it represents an instantaneous measure of market turbulences. The time period covered exhibits extremely quiet and turbulent markets at times. The change in economic conditions explains the gap between the local volatility time series and cross-sectional statistic.

This evidence suggests that cross-sections diverge strongly in nature and require individual consideration. This finding is consistent with the argument that continuous recalibration is desirable. 


\section{Parameter inference - correlations}

We next turn to the comparison of time series and cross-sectional based parameters variancecovariance matrix. The left panel of table 2.6 exhibits the time series correlations while the top right panel presents the average cross-sectional correlations. The minimum and maximum correlations are also available for the cross-sectional case, in the lower right panel. The time series and cross-sectional correlations have the same signs, yet the magnitudes are significantly different. The cross-sectional correlations are extremely high, while the time series based correlations are driven down by the noise created by the second source of variation discussed in the previous paragraph.

At the cross-sectional level, the parameters $\beta_{0}$ and $\beta_{1}$ are almost perfectly negatively correlated, with a correlation coefficient of minus one. This suggests that the relative difference between $\beta_{0}$ and $\beta_{1}$ is more relevant for capturing asymmetry rather than the values taken by these two parameters. Hentschel (1995) shows that volatility asymmetry can be modeled either as a shift or a rotation parameter. On the one hand, a shift implies that the conditional volatility response to negative shocks is higher than the response to positive shocks by a constant factor. On the other hand, rotation suggests that the conditional volatility response is more complex and requires two different slopes for responses to either positive or negative shocks. Our results shows that, for option pricing purposes, the shift parameter is the main driver of volatility asymmetry.

The cross-sectional correlation results suggest that the mean reversion parameter $\alpha$ and parameters $\beta_{0}$ and $\beta_{1}$ are also remarkably highly correlated. The dependence on past conditional volatility and past shocks are competing to generate the clustering effect needed to match the data. In a traditional GARCH $(1,1)$ sense, this implies that the pricing performance would not be appreciably altered for alternative parameter values as long as the sum of these two parameters are close to one.

We interpret these very high cross-sectional correlations as evidence that the loss function surface is flat, with many alternative local optima that are close to the global optimum. This suggests that a simple volatility specification is already over-fitting the data. Dumas et al. (1998) used the difference between in- and out-of-sample pricing errors to prove that simple ad hoc Black and Scholes models are over-fitting too. Our results show that this problem also exists for more theoretically based discrete time volatility models. These findings therefore favor the use of a parsimonious volatility specification rather than richer specification prone to over-fitting. This recommendation is aligned with the relatively good performances obtained by an asymmetric GARCH compared to richer specifications in Christoffersen and Jacobs (2004a). 


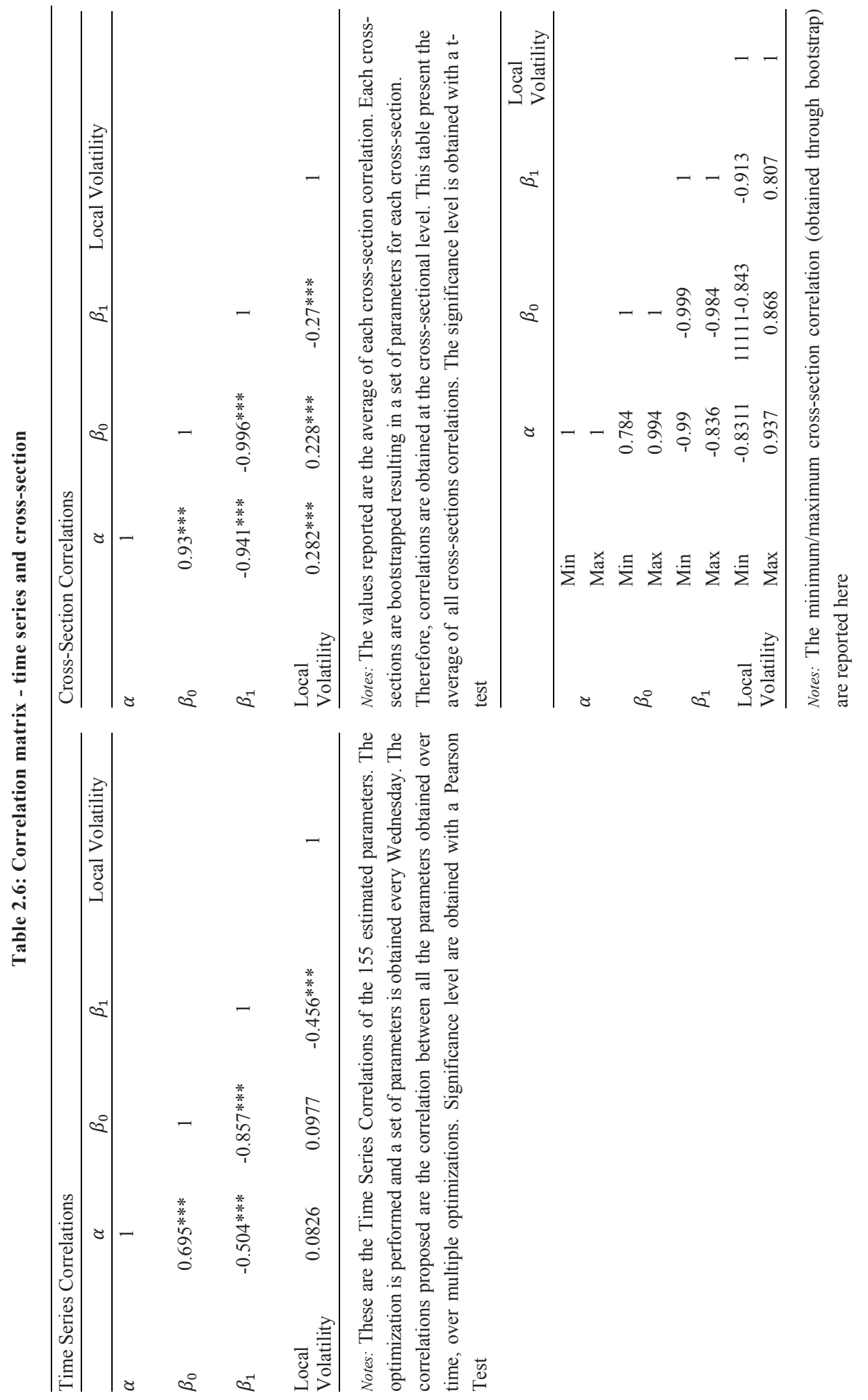


The loss function distribution function is relevant to assessing option pricing model performance. The outcome of the bootstrapping approach is an entire probability distribution of the loss function, i.e. RMSE, for each cross-section.

The variation in RMSE is unaffected by the heterogeneity between cross-sections and is specific to the individual nature of the particular cross-section.

Figure 2.2 Panel A illustrates graphically the RMSE distributions obtained for three particular cross-sections selected. The distribution around the RMSE point estimate can be truly wide, as is suggested by the cross-section of $2002 / 5 / 8$. This demonstrates that the mean of the distribution is not sufficient information for a pricing performance evaluation, and a statistical test based on the entire distribution is instead warranted.

The mean of the RMSE distribution is not the only statistic that changes between crosssections. The shape of the distribution functions also diverges strongly. Panel $\mathrm{C}$ shows, by means of example, the discrepancy of the RMSE distribution shapes for two cross-sections with an equivalent RMSE level. Table 2.7 present the results of Kolomogrov-Smirnov tests, confirming that our visual inspection is correct.

We conclude that cross-sections display significantly different levels of RMSE and diverging RMSE distribution shapes, providing additional evidence that a statistical test based on the entire distribution is to be preferred over a comparison of averages alone.

\section{Sources of time variation in RMSE}

The previous results highlight the time varying nature of the RMSE distribution. Next, we investigate the time dynamics of the RMSE distribution in a simple time series regression framework. For this analysis, a single measure to describe each RMSE distribution is needed. For this purpose, we use three different representations, being the mean of the RSME distribution, the coefficient of variation and the Asymmetric Selection Criterion (ASC), as defined in Bams, Lehnert, Wolff (2009):

$$
A S C=-\frac{1}{\overline{R M S E}} \times \frac{\overline{R M S E}-F_{2.5 \%}^{-1}(R M S E)}{\overline{R M S E}-F_{97.5 \%}^{-1}(R M S E)}
$$

where $\overline{R M S E}$ is the mean, $F_{2.5 \%}^{-1}(R M S E)$ and $F_{97.5 \%}^{-1}(R M S E)$ are the respective percentiles of the bootstrapped vector RMSE. The ASC statistic reflects a preference of negatively skewed loss distributions, implying below-average mispricing, for positively skewed counterparts. Moreover, the ASC penalizes high average and above-average RMSE while rewarding belowaverage RMSE. 

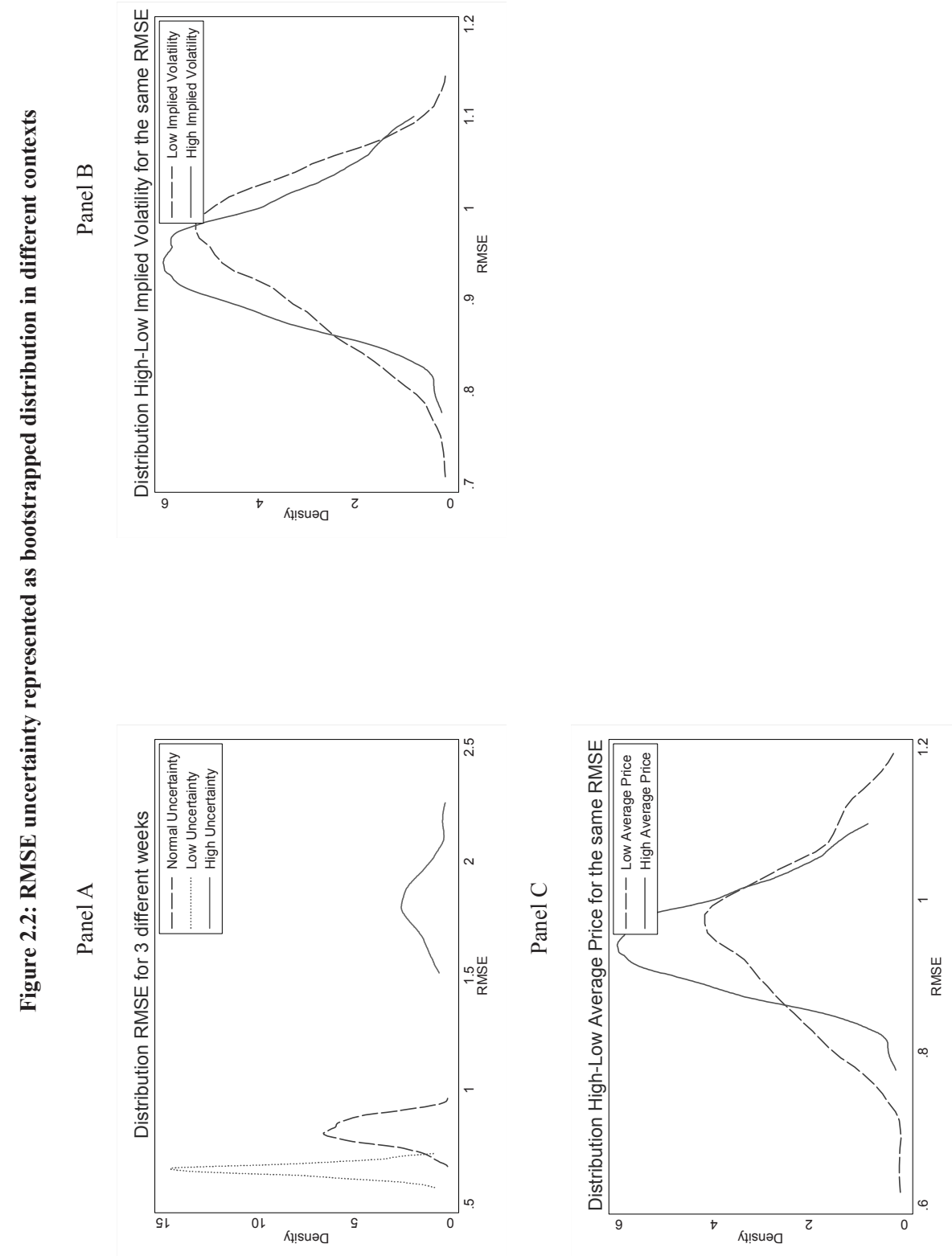

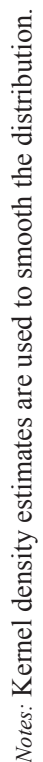


Table 2.7: RMSE distributions in different context- statistical test

\begin{tabular}{llccc}
\hline & & Mean Comparison & Variance Comparison & K-S \\
\hline RMSE & & & \\
& Normal-High & 0.000 & 0.000 & 0.000 \\
& Normal-Low & 0.000 & 0.000 & 0.000 \\
& High-Low & 0.000 & 0.000 & 0.000 \\
\hline Implied Volatility & High-Low & 0.977 & 0.154 & 0.405 \\
\hline & & & 0.12 \\
\hline Average Option Price & & 0.963 & 0.000 & \\
\hline
\end{tabular}

Notes: The table provides the P-value of alternative distribution comparison test (mean comparison test, variance comparison test, Kolomogrov-Smirnov test) for the previously displayed distributions.

Table 2.8 reports the results of time series regressions investigating the factors influencing RMSE's distribution. The three proposed measures for the RMSE distribution are regressed on three types of explanatory variables. Lagged values are used to investigate the persistence of the loss function distribution. The local volatility parameter and the leverage parameter capture the effect of market conditions. The local volatility is equivalent to the VIX. We use the parameter estimates for $\beta_{1}$ in equation (2.3) as a proxy for the risk neutral distribution skewness. Both variables are known to be measures of market turbulence and fear. The average price, the proportion of very cheap options and the average maturity capture the effect of the sample composition.

The lags are the most powerful explanatory variables. Lagged ASC and lagged coefficient of variation standalone explain respectively about $60 \%$ and $70 \%$ of the variation, as indicated by the reported R-squares for the autoregressive regressions. Moreover, the one period lagged coefficient for ASC is equal to 0.84, which suggests persistence in the short term, suggesting that the RMSE distribution of a particular cross-section is a reliable source of information for the distribution of next week's RMSE.

Market conditions affect the model pricing performance to some extent as well. The local volatility and the leverage have a significant positive effect on the change in RMSE and coefficient of variation. The leverage variable has a significant negative effect on the ASC. The results suggest that turbulent markets characterized by high volatility and negatively skewed risk neutral distributions are accompanied by considerable mispricing as well as magnified uncertainty around the RMSE. This is in line with the well-known fact that GARCH models cannot account fully for the risk neutral skewness (Barone-Adesi, Engle, and Mancini, 2008) and the CBOE VIX (Hao and Zhang, 2013). Therefore, high risk neutral skewness and VIX are coupled with a high unexplained portion of the distribution, leading to mispricing. 
Table 2.8: Cross-section pricing performance, sample composition and market conditions

\begin{tabular}{|c|c|c|c|c|c|c|c|}
\hline & \multicolumn{3}{|l|}{ ASC } & $\Delta \mathrm{RMSE}$ & \multicolumn{3}{|c|}{ Coeficient of Variation } \\
\hline & \multicolumn{4}{|c|}{ AutoregressiveMultivariateFull } & \multicolumn{2}{|c|}{ AutoregressiveMultivariate } & \multirow{2}{*}{$\begin{array}{l}\text { Full } \\
0.005\end{array}$} \\
\hline \multirow[t]{2}{*}{$\Delta$ Local Volatility } & & -0.289 & -0.073 & $0.181 * * *$ & & $0.021 * *$ & \\
\hline & & $(0.262)$ & $(0.181)$ & $(0.059)$ & & $(0.010)$ & $(0.006)$ \\
\hline \multirow[t]{2}{*}{ Leverage } & & $-1.173 * * *$ & $-0.719 * * *$ & $0.218 * *$ & & $0.060 * * *$ & $0.024 * *$ \\
\hline & & $(0.420)$ & $(0.268)$ & $(0.102)$ & & $(0.017)$ & $(0.011)$ \\
\hline \multirow[t]{2}{*}{ Average Price } & & $-0.080 * * *$ & -0.011 & $-0.012 * * *$ & & $-0.002 * * *$ & -0.001 \\
\hline & & $(0.014)$ & $(0.012)$ & $(0.004)$ & & $(0.001)$ & $(0.000)$ \\
\hline \multirow[t]{2}{*}{ Cheap Option \% } & & -1.423 & $-1.005^{*}$ & -0.003 & & $0.089 * *$ & $0.045^{* *}$ \\
\hline & & $(0.876)$ & $(0.558)$ & $(0.211)$ & & $(0.037)$ & $(0.021)$ \\
\hline \multirow[t]{2}{*}{ Average Maturity } & & 0.000 & $-0.004 * *$ & $0.003 * * *$ & & $0.000 * * *$ & $0.000 * * *$ \\
\hline & & $(0.002)$ & $(0.001)$ & $(0.001)$ & & $(0.000)$ & $(0.000)$ \\
\hline \multicolumn{3}{|c|}{ Dependant Variable Lag $0.533 * * *$} & $0.485 * * *$ & & $0.839^{* * *}$ & & $0.785^{* * *}$ \\
\hline & $(0.088)$ & & $(0.087)$ & & $(0.047)$ & & $(0.046)$ \\
\hline \multicolumn{3}{|c|}{$\begin{array}{l}\text { Dependant Variable Lag } 0.275^{* * *} \\
2\end{array}$} & $0.296^{* * *}$ & & & & \\
\hline & $(0.084)$ & & $(0.081)$ & & & & \\
\hline \multirow[t]{2}{*}{ Constant } & $0.212 * * *$ & $2.760 * * *$ & $1.349 * * *$ & $-0.281 * *$ & $0.011 * * *$ & 0.017 & -0.016 \\
\hline & $(0.070)$ & $(0.527)$ & $(0.322)$ & $(0.117)$ & $(0.003)$ & $(0.021)$ & $(0.011)$ \\
\hline Observations & 150 & 151 & 150 & 151 & 151 & 151 & 151 \\
\hline R-squared & 0.578 & 0.192 & 0.615 & 0.224 & 0.683 & 0.196 & 0.707 \\
\hline
\end{tabular}

Notes: Robust standard errors in parentheses $* * * \mathrm{p}<0.01, * * \mathrm{p}<0.05, * \mathrm{p}<0.1$

Local Volatility and RMSE appears to be non stationary based on a Dickey-Fuller test. As a precaution we take the first difference of these variables. 3 dependant variables are used ASC, $\triangle$ RMSE and Coefficient of

Variation. For ASC and Coefficient of variation 3 models are performed: a simple auto regressive model, a multivariate model and a combined model. For $\triangle \mathrm{RMSE}$ only one model is available because as a first difference this time series is not autoregressive. For ASC and Coefficient of Variation the lag length is chosen based on previous time series analysis not reported here.

The effect of including sample composition variables is limited because of the considerable multi-collinearity with the market condition variables. The regression still provides interesting insights regarding the role of sample composition on the loss function distribution. After controlling for the local volatility, the average price of a cross-section seems to slightly reduce the mispricing and the coefficient of variation. This would mean that higher prices are easier to match and to predict more precisely. However, an unconditionally higher average price implies higher volatility and worse pricing performances. A high average price is associated with high mispricing because RMSE is an absolute measure. Extremely low priced options are associated with higher RMSE distribution dispersion. 


\section{Sample composition effects}

Cheap options are often the very short maturity deep out-of-the money options. In order to change the distribution of the payoff to match the price of these options, volatility dynamics should be forced to have unrealistic, unstable parameters that would not match long term maturity options. The choice of a loss function (RMSE) targeting the absolute and not the relative pricing error will automatically leads to disregarding cheap options. Even if these options are completely $100 \%$ mispriced, this will in absolute terms still appear as a good performance, driving the RMSE down. More weight is given to matching longer term and more expensive options. As a result, in case of a loss function that uses RMSE as evaluation criterion, the informational value of cheap options is almost non-existent, while simultaneously leading to higher pricing uncertainty.

Figure 2.3: Very inexpensive options and uncertainty

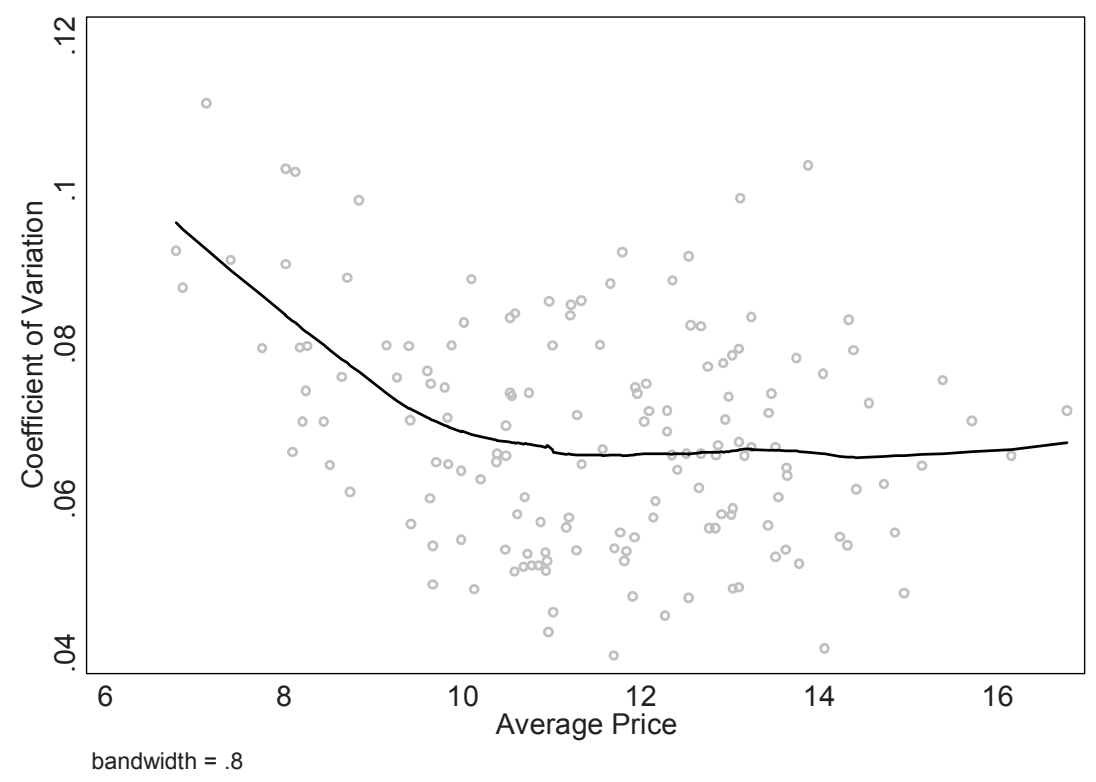

Notes: The figure presents the relationship between the coefficient of variation and Average price. The locally weighted regression of coefficient of variation on the average price line is also displayed.

Figure 2.3 explicitly pictures this effect. A high dispersion is displayed for average option prices. Certain cross-sections' average option prices are lower than $\$ 7$. These low values can be the result of a high loading in inexpensive options. The left side of the graph shows a clear negative relationship between uncertainty and average option prices. However, after a threshold at around $\$ 10$, this relationship disappears. This is evidence that the lowest average 


\section{CHAPTER 2}

price cross-sections were loaded with these inexpensive options. After the threshold, we can assume that the relationship does not hold, because the average price variation is not driven by the quantity of extremely inexpensive options. These results justify the exclusion of very cheap options and the adoption of an alternative filtering rule that allows for more options to be selected in order to have enough options per cross-section.

\section{Test example - local volatility parameter}

To illustrate this model pricing performance comparison procedure, we introduce a second model. This is the model with the same volatility dynamics as in (3), while the local volatility parameter is not treated as an unknown parameter but is fixed to the 14 days realized historical variance. Evaluating the pricing benefit of local volatility estimation is an interesting application of our framework for two reasons. First, this has not been widely studied in the previous literature (Bams, Lehnert, Wolff, 2009) and most empirical applications are silent about how they treat local volatility. Second, as suggested by previously reported parameter inference, local volatility is highly dependent on market conditions. Hence, the benefit of estimating local volatility is also likely to be time-varying.

The bootstrapped confidence intervals of both models' RMSE are used to test whether the unconstrained model has a statistically significant better pricing performance than the constrained model. Our approach is slightly time dependent since the residuals are drawn from the contemporaneous and the three previous cross-sections. To allow for this limitation we not only estimate the simpler specification but also bootstrap it, and use the mean bootstrapped RMSE as a reference for testing. This approach takes into account that, for cross-sections preceded by important pricing performance changes, the estimated RMSE and the bootstrap mean RMSE can diverge.

Figure 2.4 draws the two models' RMSE confidence intervals. The lighter grey interval represents the distribution of the constrained model's RMSE. The darker grey interval represents the distribution of the unconstrained model's RMSE. The darkest areas indicate when the two distributions overlap. Our statistical test shows that estimating local volatility significantly reduces pricing error. The underlying time series backward looking information is not a good substitute for the forward looking risk-neutral information relevant for option pricing. In $78 \%$ of the cross-sections the two RMSE's confidence intervals are distinct enough to conclude that the unconstrained model outperforms significantly at the $5 \%$ level the constrained model. However, this leaves $12 \%$ of cross-sections where the two models RMSE point estimates are within the other model's confidence intervals. Estimating the local volatility does not generate a statistically significant pricing improvement for these crosssections. Solely comparing the RMSE point estimates for these cross-sections would naturally lead to favoring the unconstrained model. Inspections of the whole RMSE distribution offer a diverging picture, stretching the importance of accounting for uncertainty. The number of 
failures to reject is substantial. Additionally these failures are not randomly distributed across time but they appear clustered. These clusters of overlapping RMSE distributions are noticeable in the middle of 2002 and at the end of 2004. This indicates that there are times when the two models' pricing performance cannot be distinguished.

Interestingly, the failures to distinguish the two models' pricing performances are concentrated during market stressed period with high volatility. The global context appears to influence the significance of the results. These times can be interpreted as either periods when the cross-sectional data is not sufficient to estimate the local volatility parameter precisely, or periods when the economic conditions suggest that the local volatility converges to the realized volatility.

Figure 2.4: Cross-sectional specification tests

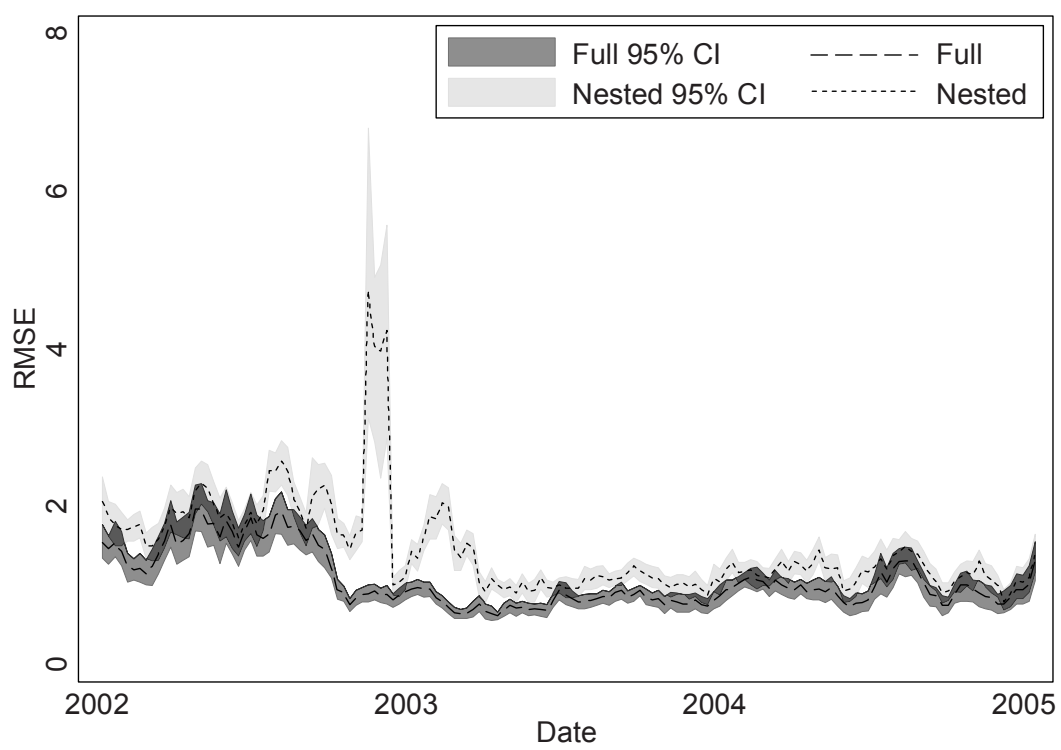

Notes: This figure is the graphic representation of the specification test. The light grey interval represents the $95 \%$ confidence interval of the nested model. The darker grey interval represents the $95 \%$ confidence interval of the full model. The darkest areas indicate where the two RMSE distributions are overlapping. A sufficient overlap is consistent with a statistical rejection of distinguishing the two models' RMSE.

The two explanations diverge, but these results are additional evidence that cross-sections are heterogeneous and require individual treatment. For that reason, option pricing models need to be evaluated, tested and benchmarked with respect to a certain informational and economic context. Our bootstrapping approach to constructing confidence intervals allows for such a comparison in a rigorous statistical manner. For instance, the conclusions of the cross- 
sectional tests are opposites for end of 2002 and for end of 2003 cross-sections. A test using multiple cross-sections from 2002 and 2003 would have yielded mixed and inconclusive results.

\subsection{CONCLUSION}

Altogether, the empirical evidence presented in this paper suggests that option cross-sections need to be regarded as independent entities. The long term cross-sectional heterogeneity affects both the pricing performance and the model selection risk. Option pricing models need to be benchmarked at the cross-sectional level to reflect the changing nature of the crosssection. Since cross-sections are similar on a short term horizon, this information is relevant for subsequent cross-sections. Nevertheless, one cannot pool too many cross-sections without running the risk of being affected by the change in the cross-sectional nature. Traditional option pricing statistical tests are inherently affected by this issue because of the substantial number of cross-sections required. Naturally, an absolute loss function comparison ignoring model selection risk and estimation uncertainty is not a better alternative.

The main novelty and innovation of our methodology is to provide a statistical framework for benchmarking models at the cross-sectional level. The bootstraps result in a loss function distribution and confidence interval around the RMSE of one specific model. The statistical significance of alternative model pricing performance differences, accounting for model selection risk, is available for each cross-section. The empirical application of our framework yields interesting results.

Firstly, we confirm the finding of Christoffersen and Jacobs (2004a), that the discrete time volatility model including clustering and simple asymmetry effect fits option data well. Richer specifications are not recommended. We use in-sample bootstrapped variancecovariance matrices to show that a simple model is already over-fitting significantly.

Secondly, we demonstrate quantitatively the considerable heterogeneity in cross-sections of options. Different cross-sections diverge strongly in nature and cannot be considered identical. This time distinctness is highlighted by the discrepancy between time series information and cross-sectional bootstrapped information. Economic conditions, sample composition and information content are causing this diversity. The differences are aggravated over time since on a short term horizon, cross-sections can be considered sufficiently homogeneous, but this does not hold for longer horizons. These results encourage the practitioners' custom of continuous recalibration, profiting from the short term persistence, but allowing for longer term variations.

Thirdly, we show that the conclusion of a specification test is cross-sectional dependent and cannot be generalized for all cross-sections. This implies that the real question to ask is not 
what model is best, but what model is the best under what conditions. Our framework permits an answer to this last question. The empirical application of this paper is limited to a specific type of discrete time volatility model, but the methodology can be applied to any class of option pricing model. 



\section{Chapter 3}

\section{On the Impact of Exclusion Filters Rules in Option Pricing ${ }^{2}$}

\subsection{INTRODUCTION}

Exclusion filtering rules are a set of criteria that determine what observations either remain in or are excluded from the calibration sample. Filtering the original dataset is the conventional first step in any empirical work on option pricing. This chapter assesses and quantifies the effect of exclusion filter rules on option pricing performance. In general, the choice of the underlying calibration sample is, next to the model selection step and the determination of the optimization criterion, a crucial step in an option pricing exercise.

A vast majority of the option literature has been dedicated to comparing the pricing performance of alternative models. This horse-race between models has taken place in various settings such as stochastic volatility models (Bakshi, Cao and Chen, 1997; Pan, 2002), discrete volatility models (Heston and Nandi, 2000; Christoffersen and Jacobs, 2004a; Frijns, Lehnert and Zwinkels, 2010) as well as across model types (Dumas, Flemming and Wahley, 1998; Lehar, Scheicher and Schittenkopf, 2002). The determination of the optimization criterion which translates into the relevance of the loss function selection for model estimation has proven to be crucially relevant as well (Christoffersen and Jacobs, 2004b; Bams, Lehnert and Wolff, 2009).

All this contrasts with the attention paid to the initial data selection process. Data selection,or the adoption of filtering rules, is acommon phenomenon in the existing empirical option pricing literature. Although proposed filtering rules usually seem intuitive, there is a lack of a systematic evaluation of the impact and appropriateness of these rules. Such an evaluation is desirable because it provides guidance on which filter rules are most appropriate for a particular option pricing exercise. The data selection process interacts with the model and loss function selection, such that the parameter estimates inherit the characteristics of the options included in the calibration sample.

${ }^{2}$ This chapter is co-authored with Dennis Bams (Maastricht University) and Thorsten Lehnert (Luxembourg University) 
The justification for data filtering resides in the information that is contained in the selected data. Certain option prices are not considered informative for the calibration of a particular model. It is beneficial to exclude these observations from the sample sinc e they would simply add noise to the estimation. From the literature, we find different approaches that are largely divergent in the way that they define and exclude uninformative option data.

One practice consists of only keeping out-of-the-money options. These options are traded more often than their in-the-money counterparts, and their prices are therefore considered to be more informative (Brandt and $\mathrm{Wu}, 2002$; Barone-Adesi, Engle and Mancini, 2008). Alternatively, Bollen and Whaley (2004) propose the exclusion of all options with an absolute delta lower than $2 \%$ or higher than $98 \%$. This takes care of implied volatilities being overly sensitive to small changes in the price, resulting from rounding errors. Third, options violating no-arbitrage principles lead to the third category of options that are commonly excluded (Bakshi et al., 1997; Neumann and Skiadopoulos, 2012).

We investigate three leading sample selection procedures proposed in the literature. The first procedure has been proposed by Bollen and Whaley (2004), BW hereafter, applying a filter based on absolute deltas. The second procedure relates to the approach used by Bakshi, Cao and Chen (1997), BCC hereafter, filtering out all put options. The third procedure is applied in Barone-Adesi, Engle and Mancini (2008), BAEM hereafter, selecting only out-of-themoney options. All three sample selection procedures are applied to the same raw dataset of European S\&P 500 index options, resulting in three different calibration samples.

We will investigate below the impact on both in-sample and out-of-sample pricing performance. In particular, we evaluate the performance at option bucket level, where a bucket is defined in terms of moneyness and maturity. Given that in all three cases we apply both the same model, i.e. the Ad Hoc Black-Scholes model, and the same optimization criterion, the discrepancies in the results are entirely attributable to the difference in data selection rules.

We empirically confirm the sizable dispersion in parameter estimates when the Ad Hoc Black-Scholes model is estimated on the three alternative sample selection methods. The accompanying impact on option pricing, resulting in diverging predicted prices is relevant. These findings are robust both in and out-of-sample, indicating that over-fitting is not a concern. Therefore, we conclude that the choice of a sample selection method is important and cannot be ignored.

Our empirical findings confirm that the best out-of-sample pricing performance is obtained by applying a filtering rule that results in a calibration sample consistent with the evaluation sample. Consistency in this context refers to option characteristics such as moneyness and maturity. In that sense, the three sample selection methods lead to different sets of 
information, and hence are efficient at filtering out uninformative observations. At the aggregate level, no sample selection procedure is dominating in either in-sample or out-ofsample pricing performance.

This is however in contrast with the clear pattern at the option bucket level. The apparent heterogeneity over option buckets triggers the desire for alternative sample selection approaches at this level. Most option pricing models are not equipped to simultaneously represent short term and long term maturity options. We find that per option bucket, a preferred sample selection approach can be identified. In particular, the sample selection approach should be careful in including those options that carry the characteristics of the particular bucket. We identify two major phenomena to account for.

First, sample selection and loss function interact. Using an absolute loss function, careful consideration should be given to the prices of long maturity options. In comparison with shorter term options, there is an implicit overweighting for long term contracts. If the primary purpose is not to price long maturity options, we recommend either including an appropriate weighting scheme for all options in the loss function, or excluding long maturity options entirely.

Secondly, after controlling for the apparent heterogeneity in maturity, the parameter divergence shrinks but persists. This is due to what we will refer to as the put-call parity anomaly. Parameters estimated from out-of-the-money call (put) options are not accurate for pricing in-the-money put (call) options. A residuals analysis suggests that the option pricing model tends to under-price long maturity puts, while long maturity calls tend to be overpriced. Although, the put-call parity violations are not exploitable once transaction costs and bid-ask spread are accounted for, the impact on parameter estimates and predicted prices is substantial.

In the industry, cross-sectional out-of-sample pricing is a common practice (Hull and Suo, 2002). For instance, Brandt and Wu (2002) estimate their model on European style options and subsequently price contemporaneous American style options. In this particular case, we recommend having the development sample matching the characteristics of the options in the evaluation sample. The same consideration applies for an out-of-time pricing exercise, when the sample composition is potentially unstable. A sudden sample composition change should be interpreted as a signal not to use the parameter estimates obtained.

\subsection{METHODOLOGY}

The analysis of the effect of different sample selections on option pricing performance requires the choice of a pricing model to be calibrated on the filtered data. For this purpose, we select the Ad Hoc Black-Scholes model, also referred to as the practitioner's Black and 


\section{CHAPTER 3}

Scholes model. This specification involves a simple parametric smoothing of the implied volatilities across moneyness and maturity.

Three reasons motivate our choice of this model. First, this model is the established benchmark for most option pricing research (Christoffersen and Jacobs, 2004b). Therefore this allows for comparability of our results. Second, this specification provides good relative pricing performances (Dumas, Fleming and Whaley, 1998). Third, for our purpose, the very ad hoc nature of the model is practical. Berkowitz (2009) shows that the Ad Hoc BlackScholes model provides an accurate approximation of a true but unknown data generating process. Following Dumas, Fleming and Whaley (1998), we use the following most complete specification of the model:

$\sigma_{i t}^{B S}=\theta_{1 t}+\theta_{2 t} \frac{S_{t}}{K_{i}}+\theta_{3 t}\left(\frac{S_{t}}{K_{i}}\right)^{2}+\theta_{4 t} T_{i t}+\theta_{5 t} T_{i t}^{2}+\theta_{6 t}\left(\frac{S_{t}}{K_{i}}\right) \times T_{i t}$

where $\sigma_{i t}^{B S}$ denotes the implied volatility of option $i$ at time $t$. The model specification represents implied volatility as a function of maturity, $T_{i t}$, and moneyness, $\left(\frac{S_{t}}{K_{i}}\right)$, where $S_{t}$ is the price of the underlying at time $t$, and $K_{i}$ is the exercise price of option i. Let $\theta_{t} \equiv$ $\left(\begin{array}{lll}\theta_{1 t} & \cdots & \theta_{6 t}\end{array}\right)^{\prime}$ denote the unknown parameters at time $t$. The fitted values for the implied volatility in equation (3.1) are plugged into the classical Black and Scholes option pricing formula to arrive at the option price:

$O_{i t}\left(\theta_{t}\right)=f^{B S}\left(\sigma_{i t}^{B S}, \frac{S_{t}}{K_{i}}, T_{i t}, R_{t}^{f}, d_{i t}\right)$

The parameters are estimated by minimizing the root mean squared errors of absolute option prices.

$R M S E_{t}=\sqrt{\frac{1}{N_{t}}\left(\sum_{i=1}^{N_{t}}\left(O_{i t}-\widehat{O_{t t}}\left(\theta_{t}\right)\right)^{2}\right)} \quad t=1, \ldots, T$

where $N_{t}$ is the number of options in cross-section $t, O_{i t}$ is the observed market and $\widehat{O_{l t}}\left(\theta_{t}\right)$ is the fitted price for option $i$ at time $t$.We rely on the root mean square error because it is the loss function selected by the greatest majority of academics and practitioners for model calibration. Also, the RMSE has desirable properties, compared to alternative loss functions, regarding out-of-sample pricing performance and estimation uncertainty (Bams et al., 2009; Christoffersen and Jacobs, 2004b). 
By $F_{t}^{B W}, F_{t}^{B C C}$ and $F_{t}^{B A E M}$ we denote the three resulting data samples after application of respectively the BW, BCC and BAEM data selection process. Subsequently, we can, by application of the procedure outlined by equations (3.1) - (3.3), arrive at parameter estimates $\theta_{t}^{B W}, \theta_{t}^{B C C}$ and $\theta_{t}^{B A E M}$, respectively.

Finally, for evaluation purposes, we introduce $R M S E_{t}\left(F_{t}^{\text {Estimation }}, F_{\tau}^{\text {Evaluation }}\right)$ as the root mean squared error that follows by estimating the parameters on sample $F_{t}^{\text {Estimation and }}$ evaluating the performance using options from the evaluation sample $F_{\tau}^{\text {Evaluation }}$. This allows us to assess the ability of parameters estimated on one sample to fit prices in another sample.

\subsection{DATA}

We use European S\&P 500 index options (SPX) from January 2002 to December 2004. The three years covered by our sample are characterized by both high and low volatility regimes. The SPX option market is the most active in the world and is popular in the option pricing literature (Barone-Adesi et al, 2008). We use closing prices of each Wednesday, leading into a data sample with 155 cross-sections.

Table 3.1: Filtering rules criteria

\begin{tabular}{|c|c|c|c|}
\hline & $\begin{array}{c}\text { Bollen and Whaley } \\
\text { (1998) }\end{array}$ & $\begin{array}{c}\text { Bakshi, Cao and Chen } \\
\text { (1997) }\end{array}$ & $\begin{array}{c}\text { Barone-Adesi, Engle and } \\
\text { Mancini (2008) }\end{array}$ \\
\hline Call / Put & & Only Call & \\
\hline Price- Min (Max) & & $\$ 3 / 8(-)$ & $\$ 0.05$ \\
\hline Maturity- Min (Max) & & $6(-)$ & $10(360)$ \\
\hline Moneyness & & & Only Out-The-Money \\
\hline Implied Volatility- Min (Max) & & & $-(70 \%)$ \\
\hline Absolute Delta- Min (Max) & $0.02(0.98)$ & & \\
\hline Arbitrage Violation Filter & NO & YES & NO \\
\hline
\end{tabular}

Notes: The table displays a set of threshold values and conditions related to different criteria to determine whether an option contract is included or excluded from the calibration sample. When a cell includes two values, this means that a minimum value and a maximum value applies for the criterion. The maximum value is presented within parentheses.

In traditional option pricing studies, a common step is to describe the filtering criteria used. In our case, we use the three sets of filters proposed by BW, BCC and BAEM. These filters are summarized in Table 3.1.

BW is the most parsimonious filter. The filter is performed on a single criterion only. This simple criterion already filters out $18 \%$ of the options from the original data sample. It is also the only data filtering approach that includes put options, call options, in-the-money options and out-of-the-money options, simultaneously. This is in marked contrast with the other data 


\section{CHAPTER 3}

filtering approaches that either select call options only or out-of-the money options only. Consequently, BW is the sample including the highest number of observations.

The most striking characteristic of BAEM is the out-of-the money only composition. This selection is justified by the higher liquidity of out-of-the money options. In addition, BAEM also diverges from $\mathrm{BW}$ and $\mathrm{BCC}$ by filtering out very long term maturity options. The other samples are not limited in that respect. The minimum option price is low in comparison. The combined effect of the maturity limit imposed and the in-the-money option exclusion leads to a substantially lower average option price. This aspect is relevant for an optimization criterion relying on an absolute loss function and for comparing alternative sample pricing errors.

Table 3.2: Sample overlap

\begin{tabular}{llll}
\hline & BW & BAEM & BCC \\
BW & $100 \%$ & $36 \%$ & $48 \%$ \\
BEAM & $71 \%$ & $100 \%$ & $35 \%$ \\
BCC & $94 \%$ & $35 \%$ & $100 \%$ \\
\hline
\end{tabular}

Notes: The table displays the percentage of options in a sample $\mathrm{x}$ also present in other samples $\mathrm{y}$. The vertical left entries are the basis samples (x) and the horizontal entries are the compared samples (y).

BCC's call options only selection relies on the put-call parity. BCC, like BAEM, imposes a minimum maturity and minimum option price. Yet the two filters differ in their cleaning approaches. While BAEM relies on out-of-the money options only, BCC is the only procedure that filters out options violating the no-arbitrage principle. Slightly more than $2 \%$ of the cleaned data in our dataset violate the no-arbitrage principle. The minimum price is higher for BCC. This is relevant since it affects the weights given to different observations. The call option only selection implies that more weights are given to deep-in-the money options because of their higher absolute prices.

Although alternative filtering criteria are divergent, they are not independent. For instance, one appealing property of delta based filtering is that it is related to implied volatility and minimum price filters. Options with an absolute delta lower than $2 \%$ or higher than $98 \%$, include a substantial proportion of options with an implied volatility higher than $70 \%$. Similarly, almost all options with a price lower than $\$ 3 / 8$ have an absolute delta lower than $2 \%$. Although the various filters are related, we still find a considerable mismatch in the resulting samples. Table 3.2 shows the proportion of overlapping options between samples. It is clear that $\mathrm{BW}$ is the broadest sample and covers most of the options found in $\mathrm{BCC}$ and BAEM. For instance, 94\% (71\%) of the options in BCC (BAEM) are also present in BW. However, the reverse is not true, since BCC and BAEM are more restrictive. Only $48 \%(36 \%)$ 
of the option found in BW are also in BCC (BAEM). BAEM is the most deviating sample. It has a smaller match with $\mathrm{BW}$ than with $\mathrm{BCC}$. Also, the biggest sample difference exists between BAEM and BCC.

The variations between the three different sample compositions warrant the investigation of two relevant issues. First, because almost all BCC and BAEM options are also present in BW, we can assess the trade-off between the benefits of large samples and the advantage of a cleaner dataset. Second, the low matching between BCC and BAEM allows for a comparison of two different option sets in fitting the volatility surface.

Table 3.3 provides summary statistics for the three samples. The high concentration of out-ofthe-money options in the BAEM sample explains the low average price. Additionally, the maximum maturity imposed by BAEM excludes expensive high time value options. The absolute average price differences suggest that pricing errors cannot be compared across samples. The distribution of prices is relevant for the model estimation step since it plays a role in the implicit weighting of each observation. The extreme prices observed in BW and BCC imply that these observations get a substantially higher weight in the estimation step. Deep in-the-money long maturity options are automatically given more weight. Such a weighting scheme is less present in BAEM, which imposes a maximum maturity and selects only out-of-the-money options.

Although the average implied volatilities are similar for the three samples, their distributions are distinct, with BW and BCC exhibiting extreme implied volatilities. The descriptive statistics suggest that BAEM provides the most selective sample. The BAEM filter, aiming at selecting the most liquid options, is also the data sample that is characterized by the highest bid-ask spread. This is explained by the low minimum price allowed by BAEM. Very cheap options are known to have relatively high bid-ask spreads.

Figure 3.1 displays the percentage of observations in each option bucket. The distribution of the sample across option buckets is strongly divergent. The BW sample is the most uniformly distributed. The biggest bucket accounts for $8 \%$ of the total population and most of the buckets include $2 \%$ to $4 \%$ of the total population. Therefore in model calibrations, BW attaches relatively equal weights to each type of option. BAEM is the most tilted towards deep out-of-the-money options. The weight given to an option in BAEM is a function of the absolute moneyness. For all maturities, deep out-of-the-money buckets always represent more than $8 \%$ of the total sample. BCC is the most tilted towards longer maturity options. The weights given to an option in BCC is a function of maturity. This relative importance, driven by the percentage of observations belonging to specific option buckets, is magnified by high prices of long maturity options, with one of the long maturity buckets even reaching $15 \%$ of the total population. 
Table 3.3: Descriptive statistics for each sample

\begin{tabular}{clccccccc}
\hline \multirow{3}{*}{ BW } & & $\begin{array}{c}\text { Number of } \\
\text { Observation }\end{array}$ & Price & IV & Call $\%$ & $\begin{array}{c}\text { Bid-Ask } \\
\%\end{array}$ & Maturity & Moneyness \\
\cline { 2 - 8 } & Mean & 56,697 & $\$ 80$ & $24 \%$ & $50 \%$ & $8 \%$ & 222 & $-1 \%$ \\
& St.Dev & & $\$ 92$ & $16 \%$ & & $10 \%$ & 198 & $19 \%$ \\
& Min & & $\$ 0$ & $6 \%$ & & $0 \%$ & 3 & $-119 \%$ \\
& Max & & $\$ 997$ & $299 \%$ & & $100 \%$ & 731 & $99 \%$ \\
\cline { 2 - 8 } BCM & Mean & 28,779 & $\$ 12$ & $24 \%$ & $49 \%$ & $33 \%$ & 135 & $-20 \%$ \\
& St.Dev & & $\$ 15$ & $10 \%$ & & $34 \%$ & 101 & $20 \%$ \\
& Min & & $\$ 0$ & $10 \%$ & & $1 \%$ & 17 & $-189 \%$ \\
& Max & & $\$ 89$ & $70 \%$ & & $100 \%$ & 353 & $0 \%$ \\
\hline & Mean & 28,998 & $\$ 87$ & $23 \%$ & $100 \%$ & $10 \%$ & 228 & $2 \%$ \\
& St.Dev & & $\$ 96$ & $13 \%$ & & $16 \%$ & 198 & $19 \%$ \\
& Min & & $\$ 0$ & $10 \%$ & & $0 \%$ & 10 & $-56 \%$ \\
& Max & & $\$ 783$ & $270 \%$ & & $100 \%$ & 731 & $195 \%$ \\
\hline
\end{tabular}

Notes: The table displays the summary statistics for the three filtered samples. The mean, standard deviation, minimum and maximum values are reported for different values. The IV refers to the Black-Sholes implied volatility. The Bid-Ask \% statistic is computed as (Ask-Bid)/Ask. Moneyness is computed as (S-K)/K for call options and (K-S)/K for put options.

\subsection{RESULTS}

In this section we report and analyse the estimation results of the Ad Hoc Black-Scholes model estimated on the three alternative samples $\mathrm{F}^{\mathrm{BW}}, \mathrm{F}^{\mathrm{BCC}}$ and $\mathrm{F}^{\mathrm{BAEM}}$. Our focus is on the pricing performance of $\theta^{B W}, \theta^{B C C}$ and $\theta^{B A E M}$ at both the aggregate level, considering all options simultaneously, and the option type level, expressed in terms of maturity and moneyness.

\section{Heterogeneity in development samples - effect on predicted prices}

In Figure 3.2, we plot the time series of parameter estimates applying the three alternative filters, $F^{B W}, F^{B C C}$ and $F^{B A E M}$. Parameter values in Figure 3.2 are time varying, which justifies relying on continuous recalibration as recommended by Christoffersen and Jacobs (2004a) and Berkowitz (2010). Parameters estimated on the same day, but based on differently filtered samples, display important variations that are significant and more accentuated for certain time periods. 
On the Impact of Exclusion Filters Rules in Option Pricing

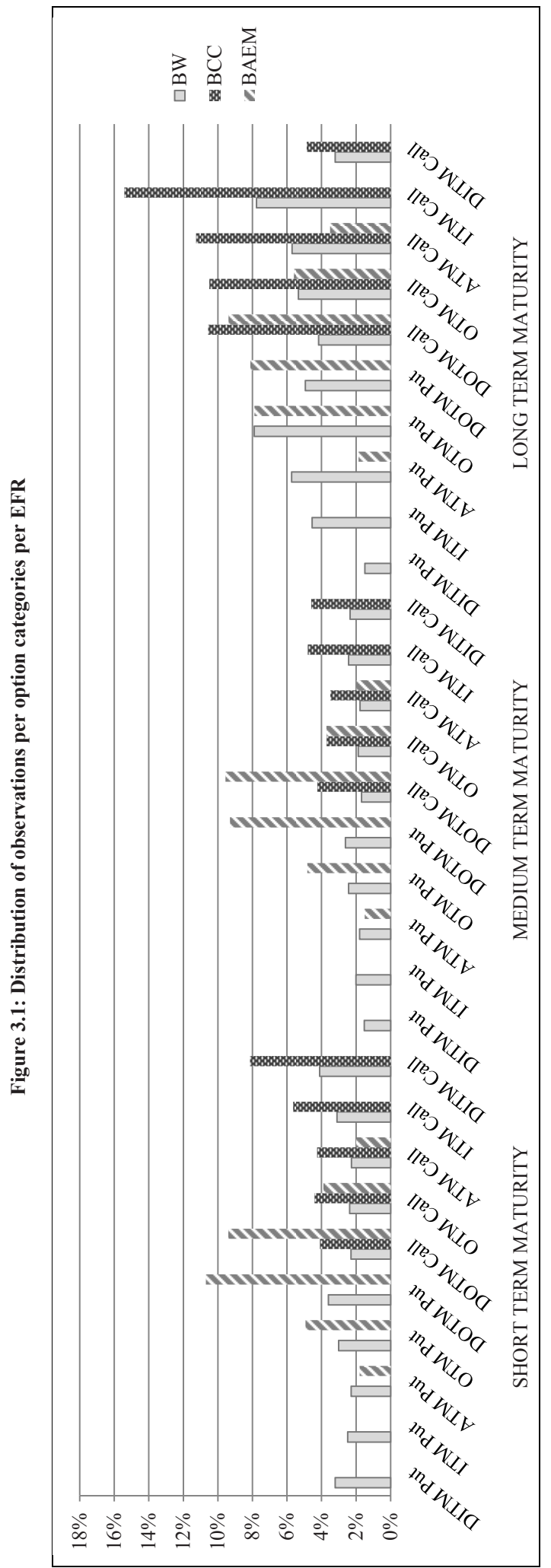

$\stackrel{g}{\Xi}$

(ิ)

昰高

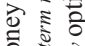

$\sum^{\circ}$

o के

$\Xi$ 政

ํ.

突

훈

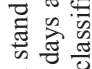

尊 8

है 胥

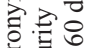

证

药 壳

的就

눈

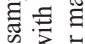

讨

본 은

일

氖高

-

氙

幽

苛

ते

家言

है वे

ธี ๑ั

흥 믈

एี

क

吾要

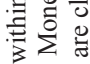

要

융ㅇㅇㅇ

훙

옹

जै 동

깅

동

o

先

范䒠

o 5

不

敦量 


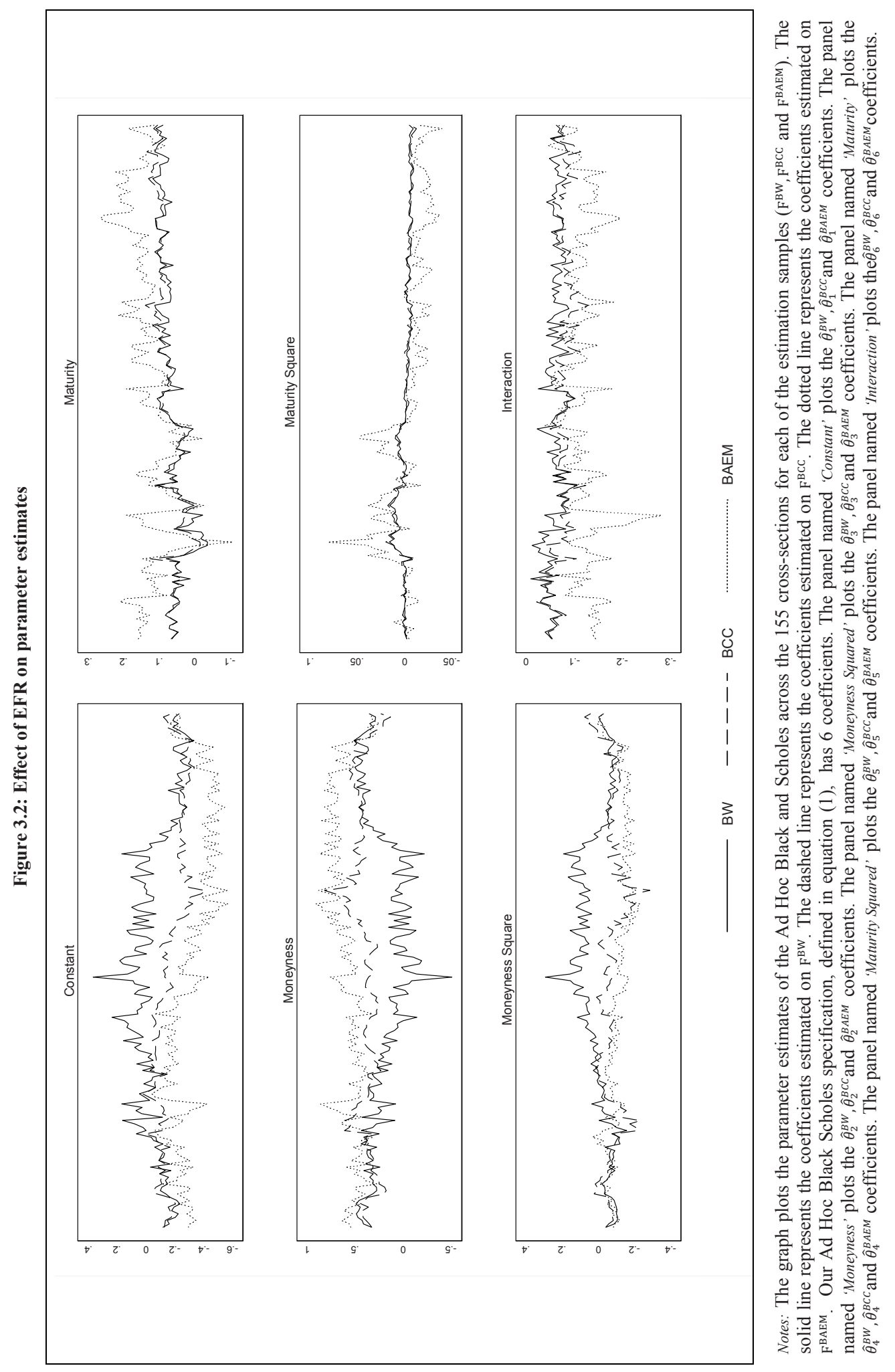


Table 3.4: RMSE across samples

\begin{tabular}{|c|c|c|c|c|}
\hline \multicolumn{5}{|c|}{ Panel A: In-sample RMSE } \\
\hline \multirow[b]{2}{*}{ Estimation } & & \multicolumn{3}{|c|}{ Evaluation } \\
\hline & & BW & $\mathrm{BCC}$ & BAEM \\
\hline \multirow[t]{2}{*}{ BW } & & 4.45 & 4.17 & 1.84 \\
\hline & & $100 \%$ & $0 \%$ & $0 \%$ \\
\hline \multirow[t]{2}{*}{ BCC } & & 5.95 & 1.03 & 1.98 \\
\hline & & $0 \%$ & $100 \%$ & $0 \%$ \\
\hline \multirow[t]{2}{*}{ BAEM } & & 7.78 & 6.06 & 0.87 \\
\hline & & $0 \%$ & $0 \%$ & $100 \%$ \\
\hline \multicolumn{5}{|c|}{ Panel B: Out-of-sample RMSE } \\
\hline \multirow[b]{2}{*}{ Estimation } & \multirow[b]{2}{*}{ Weeks } & \multicolumn{3}{|c|}{ Evaluation } \\
\hline & & $\mathrm{BW}$ & $\mathrm{BCC}$ & BAEM \\
\hline \multirow[t]{2}{*}{ BW } & 1 & 4.95 & 4.52 & 2.34 \\
\hline & & $88 \%$ & $12 \%$ & $9 \%$ \\
\hline \multirow[t]{2}{*}{$\mathrm{BCC}$} & 1 & 6.30 & 2.24 & 2.41 \\
\hline & & $11 \%$ & $86 \%$ & $20 \%$ \\
\hline \multirow[t]{2}{*}{ BAEM } & 1 & 8.10 & 6.41 & 1.67 \\
\hline & & $1 \%$ & $2 \%$ & $75 \%$ \\
\hline \multirow[t]{2}{*}{ BW } & 2 & 5.26 & 4.75 & 2.60 \\
\hline & & $82 \%$ & $14 \%$ & $11 \%$ \\
\hline \multirow[t]{2}{*}{$\mathrm{BCC}$} & 2 & 6.51 & 2.63 & 2.66 \\
\hline & & $17 \%$ & $83 \%$ & $23 \%$ \\
\hline \multirow[t]{2}{*}{ BAEM } & 2 & 8.20 & 6.51 & 2.01 \\
\hline & & $3 \%$ & $7 \%$ & $73 \%$ \\
\hline \multirow[t]{2}{*}{ BW } & 4 & 5.85 & 5.32 & 3.11 \\
\hline & & $71 \%$ & $19 \%$ & $19 \%$ \\
\hline \multirow[t]{2}{*}{ BCC } & 4 & 6.85 & 3.44 & 3.08 \\
\hline & & $27 \%$ & $76 \%$ & $29 \%$ \\
\hline \multirow[t]{2}{*}{ BAEM } & 4 & 8.39 & 6.72 & 2.58 \\
\hline & & $7 \%$ & $12 \%$ & $60 \%$ \\
\hline
\end{tabular}

Notes: The table reports the average in- and out-of-sample (1, 2 and 4 weeks) RMSEs obtained with the ad hoc Black-Scholes model. For every cross-section, the model is estimated three times on the estimation samples $\left(\mathrm{F}^{\mathrm{BW}}, \mathrm{F}^{\mathrm{BCC}}\right.$ and $\left.\mathrm{F}^{\mathrm{BAEM}}\right)$. For evaluation, we compute the RMSEs obtained with every set of parameters $\left(\theta^{B W}, \theta^{B C C}, \theta^{B A E M}\right)$ on every sample $\left(\mathrm{F}^{\mathrm{BW}}, \mathrm{F}^{\mathrm{BCC}}\right.$ and $\left.\mathrm{F}^{\mathrm{BAEM}}\right)$ resulting into a $3 \mathrm{X} 3$ evaluation results matrix. The bolded diagonal elements of these matrices indicate the results for an estimation and evaluation with the same sample. The percentages below the RMSEs show the frequency of the cross-sections where a specific combination provides the lowest RMSE. 


\section{CHAPTER 3}

Table 3.4 presents the effect of filter rules on predicted prices. The pricing performances resulting from the alternative filters are displayed. To allow for a proper comparison, i.e. using the same evaluation sample, the evaluation is done within a column. Reported pricing performance is heavily influenced by the evaluation sample characteristics.

This partially explains the disparity in the in-sample RMSEs reported by alternative studies using the Ad Hoc Black and Scholes model. Barone Adesi et al. (1998) report a 3.39\$ RMSE on SPX option while Dumas et al. (1998) and Christoffersen and Jacobs (2004b) respectively obtain average RMSEs of $\$ 0.23$ and $\$ 0.36$. Hence, the role of sample selection on pricing performances already highlights the incomparability of results across studies. Panel A shows that the RMSE is substantially minimized by using parameters consistently estimated on data cleaned with the same filters as the ones applied in the evaluation sample.The difference between the on-diagonal RMSEs and the off-diagonal RMSEs, within the same column, quantifies and highlights this effect.

These results are strikingly robust and consistent at different out-of-sample horizons. Panel B applies the parameters estimated on a particular trading day on the three filtered samples to price traded options 1, 2 and 4 weeks out-of-sample. The consistency of these results, in- and out-of-sample, emphasizes that the over-performance of the consistent estimation and evaluation is not caused by over-fitting but rather by a systematic factor affecting predicted option prices.

These out-of-sample results are different from previous empirical findings regarding loss function selection in option pricing. Similarly to our sample selection effect, the RMSE is minimized when the same loss function is applied at the calibration and the evaluation stage (Christoffersen and Jacobs, 2004b; Bams et al., 2009). Yet this divergence vanishes as the out-of-sample horizon increases.

We interpret our empirical findings as strong evidence of alternative sets of filters leading to heterogeneous sets of options. This heterogeneity in option characteristics does not allow a specific sample to represent adequately the entire universe of option contracts. The choice of filters is of the first order of importance since predicted prices depend on parameter estimates from the sample used. Therefore, the same amount of consideration should be given to the choice of a filter as is given to the choice of a model or loss function.

Since results are robust across different out-of-sample horizons, we will concentrate on the one week out-of-sample case. This horizon is consistent with the common practice of continuous re-calibration widely used both in practice and academia (Christoffersen and Jacobs, 2004a; Berkowitz, 2010). Additionally, because of the persistence observed across horizon, the features observed for the one week out-of-sample case are very likely also to be present for longer horizons pricing exercises. 
Since none of the three parameter sets are systematically dominated by their competitors, we can conclude that the three filtering rules are cleaning out noisy prices efficiently. Rather than the ability to filter out noise, our results are explained by our filters' capacity to provide good estimates for different option categories. We identify a trade-off between estimating a model locally, on a certain reduced subset of options, and globally, on all contracts simultaneously. A local estimation has obvious disadvantages.

First, following Bakshi et al. (1997), an option pricing exercise is characterized by a choice amongst miss-specified models. A local procedure is theoretically inconsistent, since an appropriate model should be able to price all options simultaneously. Secondly, the smaller number of observations implied by local estimation negatively impacts the precision of the estimated parameters and sometimes results in parameters more prone to over-fitting. Global estimation comes at the cost of a higher level of mispricing for certain options.

We identify empirically the option buckets that are properly or poorly priced from our three candidate filters. To do so, we investigate the pricing performance of alternative sets of parameters at a more granular level. Table 3.5 presents the one week out-of-sample pricing performance at the option bucket level.

Interestingly, the results obtained at the bucket level are different from the previously reported performance results at aggregate level. The diagonal of the RMSE matrix no longer contains the smallest figures, suggesting that a consistent estimation and evaluation approach at the option bucket level does not necessarily yield the best pricing performance.

Table 3.6 provides an intuitive summary of table 3.5, indicating the 'best' filtering method to adopt for each option bucket. Three possible situations arise in the overview. First, no filter is dominant. This situation is rare and is denoted by '-'. Second, one filter is viewed as the preferred filter to be adopted for a particular option bucket. This filter always provides both the lowest average RSME and lowest RMSE in the majority of the cross-sections. Third, if in addition this filter always provides the lowest average RSME and the best results in 50\% or more of the cross-sections, we consider it to be a strong domination and indicate it by a star.

Table 3.6 shows that the relative filter performance differs both with maturity and moneyness. The results suggest that $\theta^{B A E M}$ is the best alternative to price all short maturity options. Although, $\theta^{B A E M}$ also provides satisfactory results for medium maturity options, $\theta^{B W}$ and $\theta^{B C C}$ are preferred in three of the short maturity buckets. In case of long maturity options, $\theta^{B A E M}$ turns out to be the worst. Long maturity calls are remarkably well priced by $\theta^{B C C}$, whereas $\theta^{B W}$ provides the best predicted prices for most of the long maturity puts. 


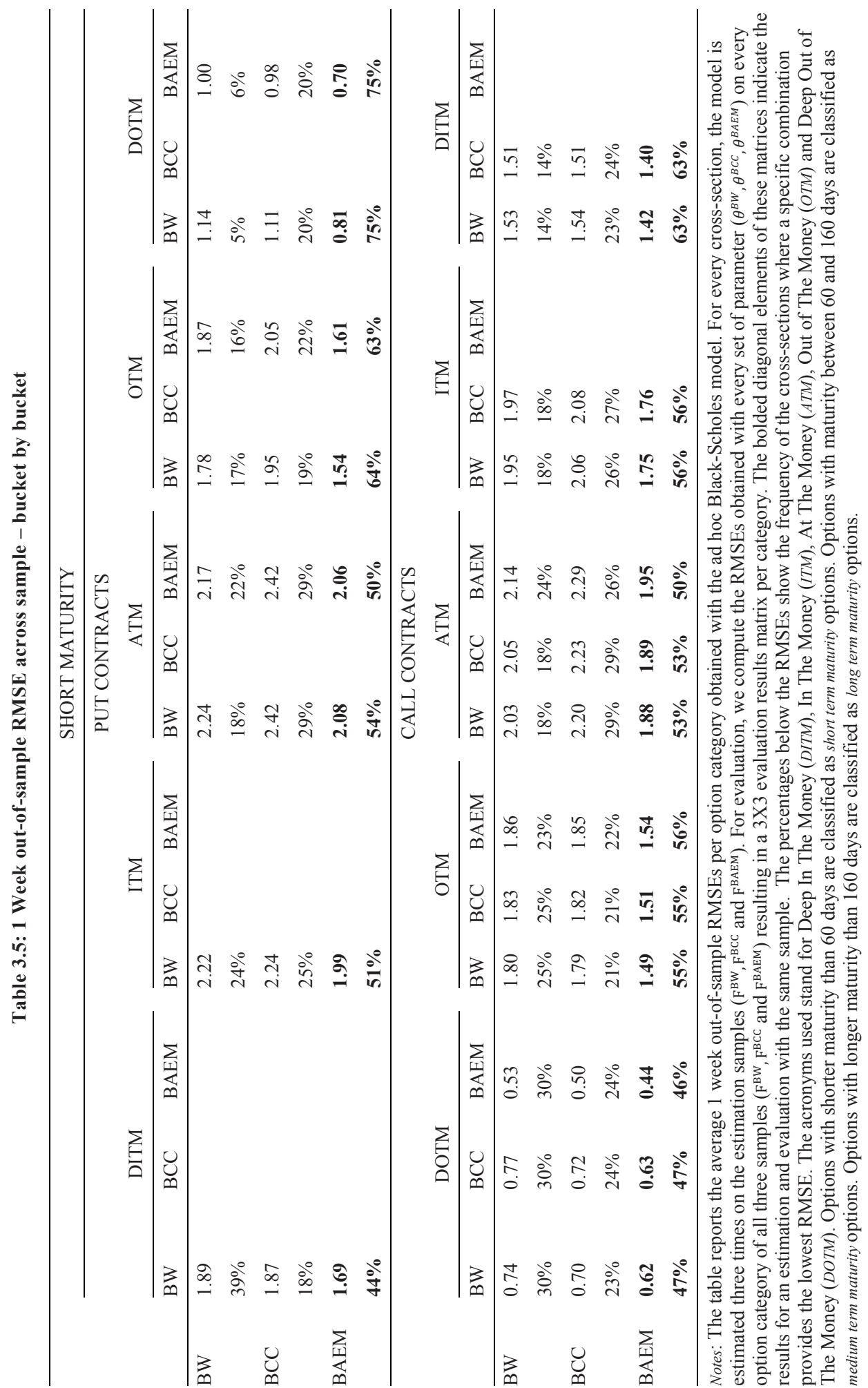




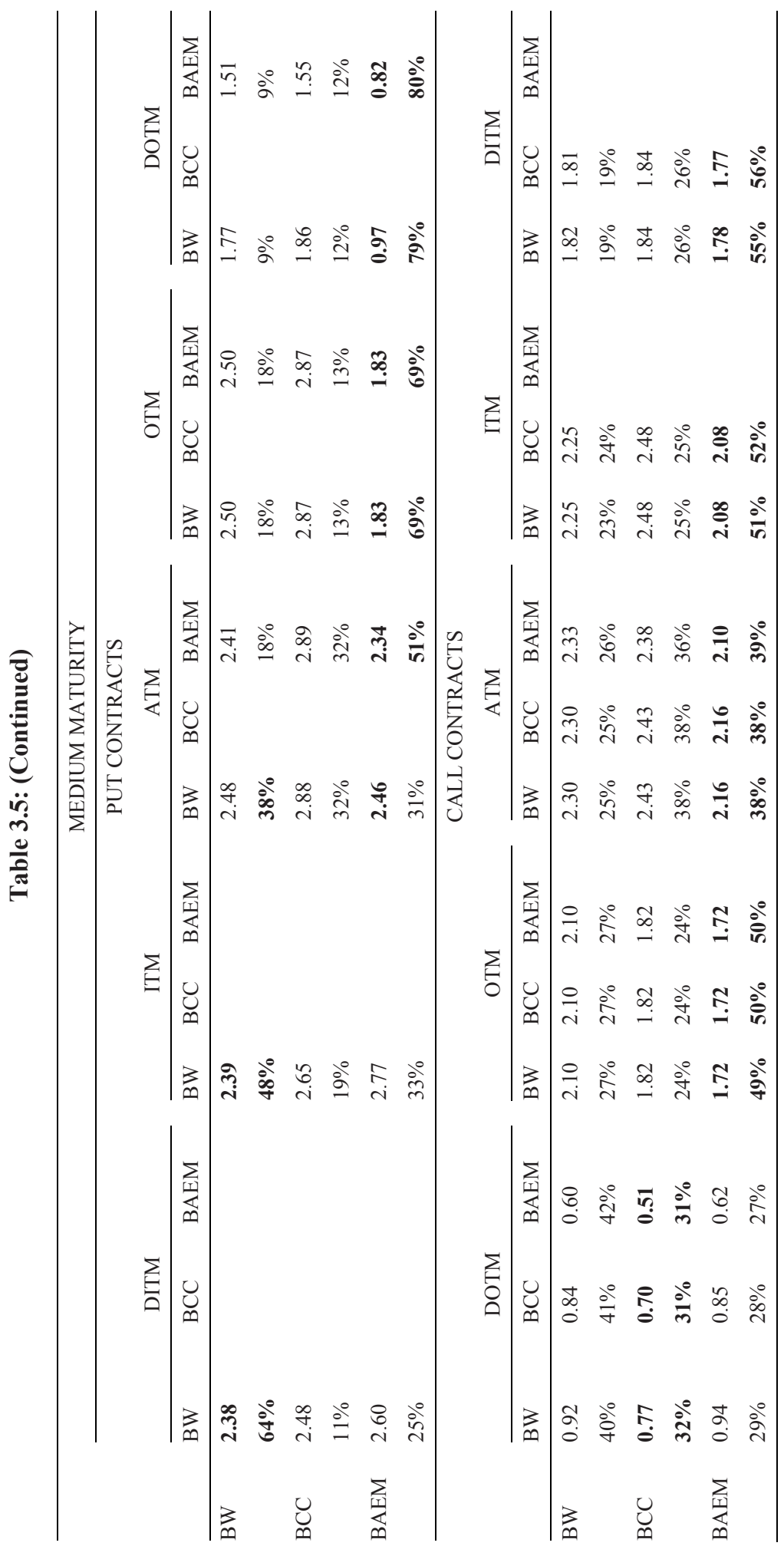




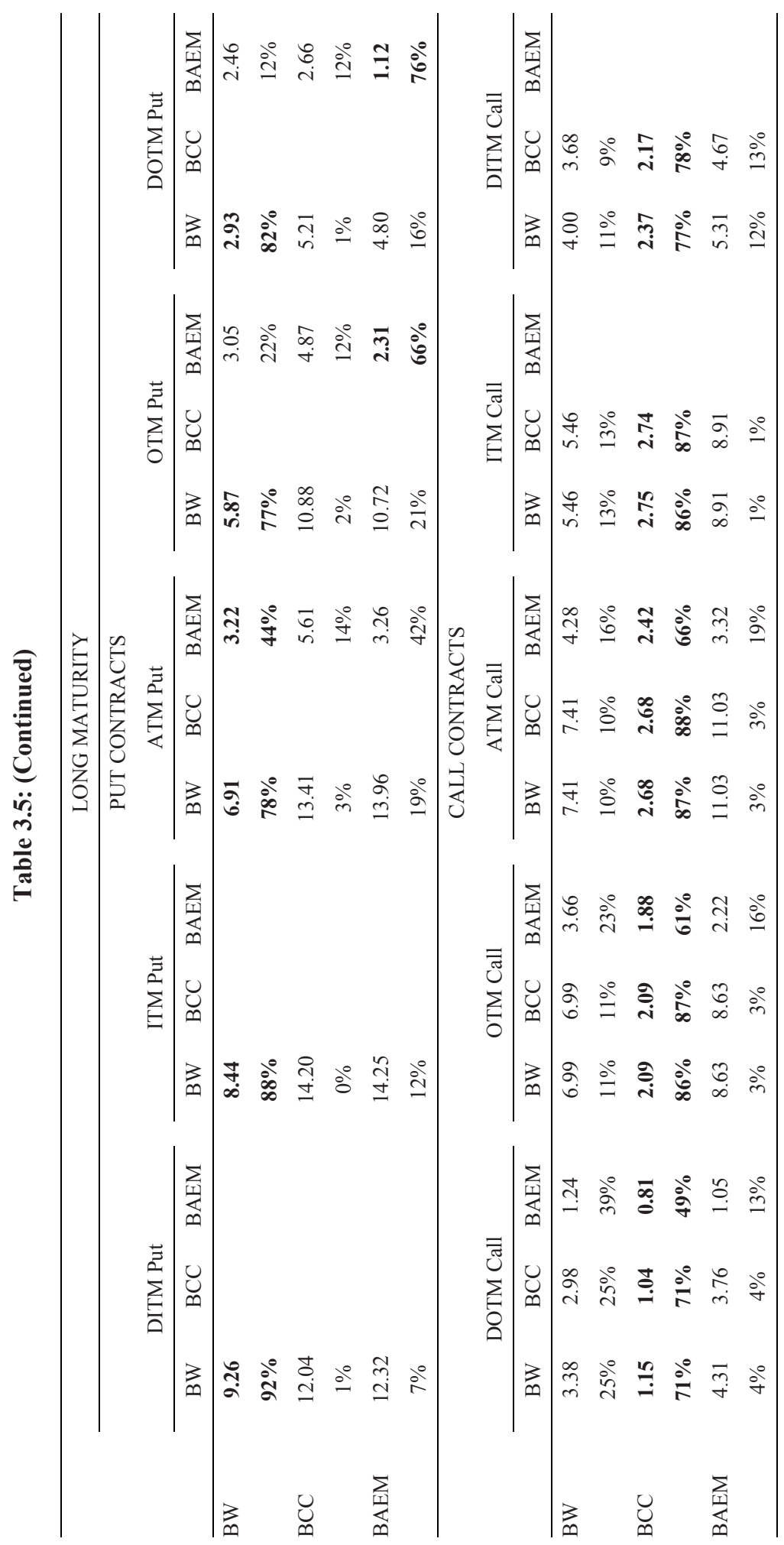


Table 3.6: Best filter

\begin{tabular}{|c|c|c|c|c|}
\hline \multirow{2}{*}{\multicolumn{2}{|c|}{ CONTRACT TYPE }} & \multicolumn{3}{|c|}{ MATURITY } \\
\hline & & \multirow{2}{*}{$\begin{array}{l}\text { SHORT } \\
\text { BAEM }\end{array}$} & \multirow{2}{*}{$\begin{array}{l}\text { MEDIUM } \\
\mathrm{BW}^{*}\end{array}$} & \multirow{2}{*}{$\begin{array}{l}\text { LONG } \\
\text { BW* }^{*}\end{array}$} \\
\hline & DITM & & & \\
\hline & ITM & BAEM* & $\mathrm{BW}^{*}$ & $\mathrm{BW}^{*}$ \\
\hline \multirow{5}{*}{ PUT } & ATM & BAEM* & - & BW \\
\hline & OTM & BAEM* & BAEM* & - \\
\hline & DOTM & BAEM* ${ }^{*}$ & BAEM* & - \\
\hline & DOTM & BAEM & $\mathrm{BCC}$ & $\mathrm{BCC}$ \\
\hline & OTM & BAEM* & BAEM & BCC* \\
\hline \multirow[t]{3}{*}{ CALL } & ATM & BAEM* ${ }^{*}$ & BAEM & BCC* \\
\hline & ITM & BAEM* & BAEM* & BCC* \\
\hline & DITM & BAEM* & BAEM* & BCC* \\
\hline
\end{tabular}

Notes: The table is a summary of the previous results. It indicates what the best filter is to apply for estimation purposes in order to price options from a given category. A filter is defined as 'best' when it always provides the lowest average RMSEs for a category across every evaluation sample. '-' means that there is no absolute best filter to price this given option category. ' $*$ ' indicates that the best filter also provides the lowest RMSE in more than $50 \%$ of the cross-sections.

\section{Sources of heterogeneity}

We identify two main drivers underlying the heterogeneity in option samples. Both the option maturity and the put versus call filtering criteria explain an important proportion of the predicted price differences between filters.

Both Table 3.4 and 3.6 highlight the importance of time to maturity. Table 3.4 shows that $F^{B A E M}$ is always the worst alternative to price $F^{B W}$ and $F^{B C C}$ options. Furthermore, $F^{B A E M}$ options are poorly fitted by the option pricing model based on either $F^{B W}$ or $F^{B C C}$. Similarly, Table 3.6 shows a clear dichotomy between short and long maturity options. Short maturity buckets are, in terms of pricing performance, overly dominated by $F^{B A E M}$, while for options in long maturity buckets the exact opposite is the case. We relate this finding to the fact that $F^{B A E M}$ is the only filter with a maximum maturity imposed. Contracts with maturity higher than 360 days are excluded. To assess the impact of maturity filtering, we replicate the one week out-of-sample pricing exercise imposing the same maturity constraint on all three samples $\mathrm{BW}, \mathrm{BCC}$ and BAEM.

From Table 3.7 it follows that after the maturity adjustment, pricing performance across the three alternative filters converges. Likewise, option pricing based on BEAM is no longer the 
worst alternative to fit option prices with $\mathrm{BW}$ or $\mathrm{BCC}$ characteristics. Hence, the inclusion of a maturity limit has a significant impact. Filtering rules including long maturity options interact with the absolute difference loss function, attaching more weight to these options. This finding supports the argument made by Broadie, Chernov and Johannes (2007) and Kanniainen, Lin and Yang (2014), who argue that absolute loss functions impose a higher weight on expensive options. Accordingly, if long term option pricing is not the main objective, we recommend excluding these contracts from the sample used for the model calibration.

Table 3.7: RMSE across sample maturity adjusted (out-sample pricing 1 week)

\begin{tabular}{llll}
\hline & BW* & BCC* & BAEM \\
BW* & $\mathbf{2 . 8 5}$ & 2.54 & 2.94 \\
& $\mathbf{( 6 8 \% )}$ & $(30 \%)$ & $(6 \%)$ \\
BCC $^{*}$ & 3.34 & $\mathbf{2 . 0 0}$ & 2.54 \\
& $(21 \%)$ & $\mathbf{( 6 8 \% )}$ & $(16 \%)$ \\
BAEM & 3.21 & 2.61 & $\mathbf{1 . 6 7}$ \\
& $(15 \%)$ & $(24 \%)$ & $\mathbf{( 7 9 \% )}$ \\
\hline
\end{tabular}

Notes: The table reports the average 1 week out-of-sample RMSEs obtained with the ad hoc Black-Scholes model. For every crosssection, the model is estimated three times on the estimation samples $\left(\mathrm{F}^{\mathrm{BW} *}, \mathrm{~F}^{\mathrm{BCC} *}\right.$ and $\left.\mathrm{F}^{\mathrm{BAEM}}\right)$. For evaluation, we compute the RMSEs obtained with every set of parameter $\left(\theta^{B W^{*}}, \theta^{B C C^{*}}, \theta^{B A E M^{*}}\right)$ on every sample $\left(\mathrm{F}^{\mathrm{BW}}, \mathrm{F}^{\mathrm{BCC}}\right.$ and $\left.\mathrm{F}^{\mathrm{BAEM}}\right)$ resulting into a $3 \mathrm{X} 3$ evaluation results matrix. The bolded diagonal elements of these matrices indicate the results for an estimation and evaluation with the same sample. The percentages below the RMSEs show the frequency of the crosssections where a specific combination provides the lowest RMSE.

Another source of heterogeneity relates to the different impact of call options versus put options in the calibration sample. From table 3.6 it follows that $F^{B C C}$ is the best filter to price every call option category, but provides a notably poor pricing performance for all the put option categories. This empirical fact is inconsistent with the put-call parity axiom. Although put-call parity violations are seldom exploitable (Cremers and Weinbaum, 2010), they are not rare. These prices mismatches are observed for individual equity options (Atilgan, 2010), as well as for European index options (Wagner, Ellis and Dubofsky, 1996; Amin, Coval and Seyhun, 2004).

Table 3.8 presents, in the case of the $\mathrm{BCC}^{*}$ sample, the put-call parity violations, measured as the difference in implied volatility between a call and its matching put. While no significant dispersion is observed for low maturity options, medium and long maturity puts are overpriced compared to long maturity calls. Wagner et al. (1996) also show that violations are more frequent for longer maturity options. Consequently, the case of parameter calibration on both puts and calls will face a trade-off between under-pricing puts and over-pricing calls. 
Alternatively, a model that is only calibrated on puts (calls) will price puts (calls) very well at the expense of the ignored calls (puts).

Table 3.8: Put-call parity deviation per maturity

\begin{tabular}{llll}
\hline & Short Maturity & Medium Maturity & Long Maturity \\
\hline Mean & $0.02 \%$ & $-0.41 \%$ & $-0.72 \%$ \\
StDev & $5.21 \%$ & $2.15 \%$ & $1.63 \%$ \\
Skewness & -0.26 & -0.42 & -1.18 \\
Kurtosis & 35.08 & 6.74 & 7.99 \\
Percentiles & & & \\
$1 \%$ & $-13.06 \%$ & $-7.05 \%$ & $-6.36 \%$ \\
$5 \%$ & $-6.14 \%$ & $-3.96 \%$ & $-3.57 \%$ \\
$10 \%$ & $-3.98 \%$ & $-2.77 \%$ & $-2.62 \%$ \\
$25 \%$ & $-1.68 \%$ & $-1.37 \%$ & $-1.35 \%$ \\
$50 \%$ & $-0.22 \%$ & $-0.32 \%$ & $-0.53 \%$ \\
$75 \%$ & $1.59 \%$ & $0.76 \%$ & $0.22 \%$ \\
$90 \%$ & $4.07 \%$ & $1.78 \%$ & $0.92 \%$ \\
$95 \%$ & $6.52 \%$ & $2.80 \%$ & $1.41 \%$ \\
$99 \%$ & $16.93 \%$ & $5.31 \%$ & $2.86 \%$ \\
\hline
\end{tabular}

Notes: The table provides the summary statistics on the put-call parity deviations within the option sample used. The dispersion measures as the implied volatility spread $\left(\sigma_{\text {Call }}^{\mathrm{IV}}-\sigma_{\mathrm{Put}}^{\mathrm{IV}}\right)$ between a call and a put with the same maturity, same moneyness, at the same date. The dispersions are summarized per category of option maturity.

In order to evaluate the impact of put-call parity violations on our results we analyse the residuals obtained from $\mathrm{F}^{\mathrm{BW} *}$ priced with $\theta^{\mathrm{BW} *}$ for three maturity horizons. Consistent with table 3.8, the put and call residual distributions are virtually indistinguishable and centred around zero for short maturity options. For the longest maturity contracts, the put and call residual distributions are different. We confirm that puts are underpriced while calls are overpriced. The small implied volatility divergences between puts and calls have a significant impact on parameter estimates and on the accompanying predicted prices. Assuming that a call only sample is representative for the puts or that an out-of-the-money only sample is representative for the in-the-money options is not possible.

\subsection{CONCLUSION}

A considerable amount of attention has been given to model selection and loss function selection in option pricing. We show that data selection, or the determination of exclusion filtering rules, is also a relevant part of the model specification since it affects predicted prices in a non-random manner. Our empirical application highlights the finding that no single 
filtering procedure is optimal. Instead, each filtering procedure is specialized at predicting accurately a particular category of option prices at the expense of other categories. For the empirical option pricing exercise, we recommend paying more attention to the ex-ante data selection procedure. The filter used, and its effect on predicted prices, should be aligned with the objective of the model. Two types of filters have important implications. The cut-off point for the inclusion of maximum maturity option is of the first order of importance, since the inclusion of long maturity contracts strongly impacts the parameter estimates. Also, deviation from the put-call parity means the two types of contracts are not a valid substitute for one another. For this reason, we advise the inclusion of put and call as well as in and out of the money contracts in the development sample. 
Figure 3.3: Put Vs Call residuals
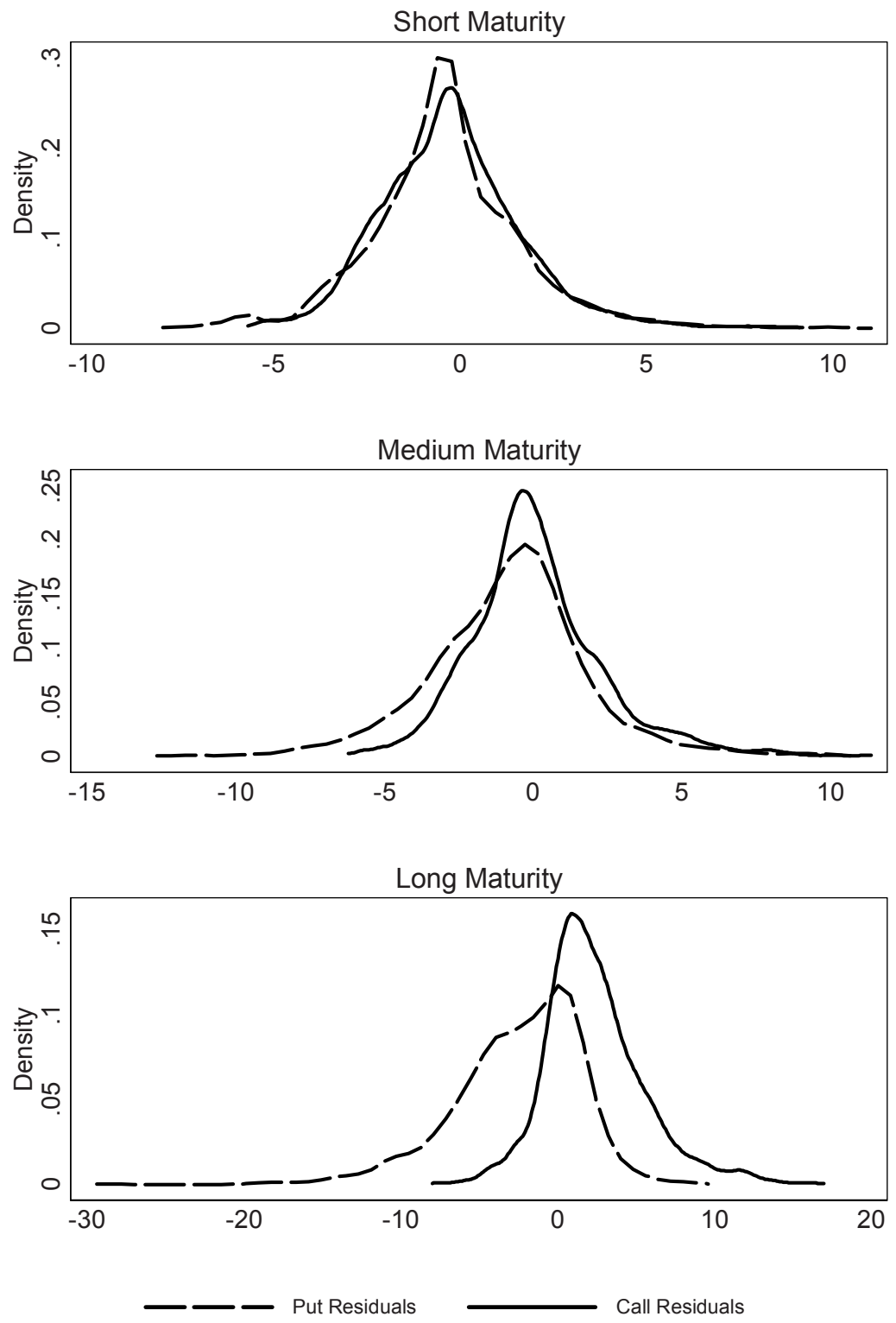

Notes: The figure displays the $\mathrm{F}^{\mathrm{BW} *}$ put and call residuals distributions resulting from the option prices predicted with the ad hoc Black and Scholes model and the $\theta_{B W_{*}}$ estimated parameters. The distributions are presented per category of option maturity. 



\section{Chapter 4}

\section{The Impact of Uncertainty in the Oil and Gold Market on the Cross-Section of Stock Returns ${ }^{3}$}

\subsection{INTRODUCTION}

In this chapter, we bridge the gap between two major strands of literature in financial economics. The first strand relates to the effect of uncertainty on asset prices (Bansal and Yaron, 2004; Anderson, Ghysels and Juergens, 2009; Bekaert, Engstrom and Xing, 2009). The second stream focuses on the spillover effect of commodity prices on the equity market (Jones and Kaul, 1996; Killian and Park, 2009). The latter research theme has generated voluminous evidence of a relationship between oil prices and the equity market (Driesprong, Jacobsen and Maat, 2008; Narayan and Sharma, 2011). To the best of our knowledge, we are the first to investigate and to empirically demonstrate the substantial role played by uncertainty in alternative asset markets for the equity market.

Knight (1921) draws out theoretically the importance of differentiating risk from uncertainty. This distinction originates from the fact that risk is measurable, whereas uncertainty is not. Accordingly, economic agents and investors have different attitudes towards these two concepts. Experimental evidence suggests that individuals have different aversions towards risk and uncertainty (see e.g. Ellsberg, 1961 and Anderson et al., 2009). Thus, uncertainty should play a prominent and separable role in financial markets. According to the theoretical model of Bansal and Yaron (2004), uncertainty and investor uncertainty aversion are the components required to explain many features in financial data previously considered as anomalies. A more recent strand of literature has empirically demonstrated the strength and the multifaceted nature of the connexion between uncertainty and financial markets. Bekaert et al. (2009) show that uncertainty, proxied by the conditional variance of cash flow growth, explains volatility of financial markets and, to a lesser extent, the equity premium. Anderson et al. (2009) provide evidence of a strong relationship between uncertainty and expected return. Using professional forecasters' disagreement about their macro-economic and profit expectations as a measure of market-wide uncertainty, they show that uncertainty affects both stock returns in the time series dimension and the cross-section of expected returns. They

\footnotetext{
${ }^{3}$ This chapter is co-authored with Dennis Bams (Maastricht University), Iman Honarvar (Maastricht University) and Thorsten Lehnert (Luxembourg University)
} 
elaborate that the uncertainty explains expected return distinctively from risk. Bali and Zhou (2014) confirm that higher exposure to uncertainty is related to higher expected return.

Buraschi, Trojani and Vedolin (2014) suggest that uncertainty appears to be a market-wide factor, since it is also found to affect the bond market by increasing credit spreads. Connolly, Stivers and Sun (2005) cast light on how uncertainty affects asset allocation. They show that periods of high uncertainty are characterized by higher bond returns compared to stock returns. Uncertainty is accompanied by a flight-to-safety phenomenon, causing the stock-bond correlation to drop. Baele, Bekaert and Inghelhecht (2010) also confirm that flight-to-safety is of the first order of importance in explaining stock and bond co-movement. The large amount of evidence regarding the impact of uncertainty on financial market broadens and highlights the importance of gaining deeper knowledge about different sources of uncertainty.

Concurrently, a significant amount of effort has been devoted to evaluating how other markets, e.g. the commodity markets, have an effect on the equity market. In that respect, the most studied market under investigation has probably been the oil market. Since crude oil came under the control of the OPEC, it has started experiencing significant fluctuation in prices. Oil price changes strongly impact the global economy (Driesprong et al., 2008). According to Hamilton's (1983) results and interpretations, high oil prices have contributed to some of the major US economic recessions. A similar negative relationship is also found between oil shocks and aggregated stock market returns. Jones and Kaul (1996) document this relationship for US, Canadian, UK and Japanese stock markets. They show that the relationship is mainly channelled through a change in real cash flows rather than changes in expected returns. Narayan and Sharma (2011) confirm this hypothesis at the individual stock return level. Furthermore, Driesprong et al. (2008) show that changes in oil prices significantly and economically forecast subsequent stock returns.

The major effect of oil on economic activity has also motivated certain studies to include the oil price as a risk factor (Chen, Roll and Ross, 1986; Ferson and Harvey, 1993). These studies do not provide strong evidence that oil price changes are a systematic risk factor. However, the results may have been impaired by the fact that not all oil price shocks are relevant for the equity market. Killian and Park (2009) provide evidence that oil demand-driven shocks are the most relevant type of oil price changes for equities. Demand shocks are mainly caused by increased uncertainty about oil supply. Their results suggest that oil price changes affect both dividend growth and discount factors. This evidence reflects the disagreement in the literature as to whether oil is a priced risk factor.

We do recognize the importance of oil price changes for stock markets, but we argue that uncertainty about future oil prices is more prone to affect the value of equities. As suggested by Killian and Park (2009), uncertainty about oil supply and the future price of oil is a major concern for firms' future cash flows and required rate of return. Considering both their 
argument and the strong evidence presented on the impact of uncertainty on equity markets, this paper attempt to isolate the oil price uncertainty and its effect on equity prices and equity valuation. To date, we have not seen any study in the literature attempting to investigate this channel.

For the sake of completeness in our study, we also investigate the impact of stock market uncertainty and gold market uncertainty on equity market. While the first type of uncertainty appears as a natural benchmark, the introduction of gold uncertainty is motivated by the recent evidence of the linkages between stock and gold markets under stressed market conditions (Chan, Treepongkaruna, Brooks and Gray, 2011). Gold has been found to be a safe haven asset during the recent financial crisis (Baur and McDermott, 2010), and it responds negatively to good macro-economic news (Elder, Miao and Ramchander, 2012). Because of gold's negative exposure with the stock market, it is interesting to assess the impact of gold market uncertainty.

One major challenge is to obtain a good measure of uncertainty for each of those markets. Anderson et al. (2005) pointed out the limitations of relying on analysts' forecasts dispersion. They conclude that because of analysts' optimism (pessimism) on long (short)-term forecasts, agency issues and behavioral biases, beliefs disagreements cannot be a perfect proxy. In addition, they note that similar education, goals and interactions prevent the diversity of analysts' forecasts from being a generic survey of disagreement in the whole economy, compared with more diverse participants. Essentially, the measures relying on forecasts dispersion suffer from three main drawbacks. First, they are not available at the high frequency required to investigate stock price movements and correlation. Second, they show uncertainty amongst a few forecasters rather than the uncertainty perceived by the overall market. And, finally, the analysts forecasting macro-economic variables, earnings, oil and gold prices cannot be assumed to be homogenous. Consequently the uncertainty measures based on their forecasts would not be comparable across markets.

Instead, we rely on the volatility risk premium as proxy for uncertainty, as has recently been suggested in the literature. Following the methodology of Bakshi, Kapadia and Madan (2003), the volatility risk premium is measured as the difference between the realized volatility and risk-neutral volatility implied by S\&P, oil and gold options. The volatility risk premium is not the expected volatility but is a measure of the price of a hedge against a change in the volatility. Option prices reflect the underlying distribution expectations from investors. When option traders are uncertain about the shape of this distribution, option prices increase further to reflect this additional uncertainty. In fact, options can be seen as a hedge against uncertainty or change in the underlying distribution. Intuitively, when the distribution of returns is uncertain, the volatility risk premium is higher. Carr and $\mathrm{Wu}$ (2009) measure return variance uncertainty with the variance risk premium, while Bali and Zhou (2013) use the variance risk premium as a proxy for market-wide uncertainty. They also demonstrate that this 


\section{CHAPTER 4}

proxy for uncertainty correlates highly with various other uncertainty proxies such as the conditional variance of the Chicago Fed National Activity Index and the conditional variance of the growth rate of industrial production. Moreover, the strong link found empirically between individual equity option volatility risk premium and analyst disagreement by Buraschi, Trojani and Vedolin (2014), gives us confidence that the volatility risk premium is an appropriate measure of uncertainty.

We find that there is a common component across uncertainties of different asset markets. A systematic uncertainty factor, related to representing the overall economic uncertainty, affects stock, oil and gold markets simultaneously. The significant positive correlations between these three markets' volatility risk premia and their volatility risk premia innovations support this interpretation. However, these correlation values remain low and indicate that an assetspecific uncertainty factor exists. For instance, in the case of oil, political instability could be an important asset-specific uncertainty factor.

We test for the relationship between market uncertainty and stock returns along two dimensions. In a time series setting, we find that uncertainty negatively affects stock returns. An increase in uncertainty, proxied by a negative innovation in the volatility risk premium, is contemporaneously related to negative returns in a significant proportion of the stock universe. Conversely, very few stocks are positively impacted by uncertainty shocks and would provide a good hedge against changes in uncertainty. A comparison of the role of our three sources of uncertainty shows that S\&P uncertainty has a dominant effect on equity prices: 21.6 percent of the stock universe is negatively affected by the stock market uncertainty while only 12.5 percent and 15.6 percent of the stocks are negatively affected by the oil and gold uncertainty, respectively. Although oil and gold uncertainty influences a smaller number of stocks, this effect is robust. We show that the role of oil and gold uncertainty is explained neither by oil and gold returns nor by our systematic uncertainty factor, i.e. the S\&P uncertainty. The result reveals that oil and gold-specific uncertainty also matters in the time series of stock returns. Having shown that stock returns are exposed to changes in uncertainty, we test whether uncertainty is priced in the cross-section of expected returns. Uncertainty-averse investors require extra compensation for holding assets that are positively correlated with systematic uncertainty innovations. We evaluate and compare the premium obtained for exposure to oil and gold uncertainty with that obtained for exposure to S\&P uncertainty. We form five portfolios independently sorted on their past exposures to S\&P volatility risk premium innovations, five portfolios independently sorted on their past exposures to oil volatility risk premium innovations and five portfolios independently sorted on their past exposures to gold volatility risk premium innovations. Our empirical results show that loadings on oil and gold factors cannot explain stocks expected returns. In contrast, exposure to S\&P uncertainty is priced within and across industries. The high exposure minus low exposure portfolio delivers a monthly statistically significant Carhart four factors alpha of 78 basis points. This result is consistent with the finding of Bali and Zhou (2013), who use 
variance risk premium as an uncertainty measure, but also with Anderson et al. (2009), who take analysts forecast dispersion as uncertainty proxy.

The difference in premia obtained from exposure to oil and gold uncertainty and exposure to S\&P uncertainty are stable through time. Oil and gold uncertainty are priced neither in expansion nor in recession. However, S\&P uncertainty is priced under both economic states.

An intra-industry investigation reveals interesting results for oil. While oil uncertainty is not priced for the whole market, it is priced within oil relevant industries. The most oil uncertainty exposed firms within these industries are compensated by substantially higher returns than the least exposed firms within the same industries. An economic interpretation of this result suggests the market segmentation between industries. The existence of specialized industry investors (Hong, Torous and Valkanov 2007), holding undiversified portfolios, can cause a specific factor to be priced within an industry. Also, specialized investors cause industry relevant news, such as oil news, to be more quickly reflected in certain industries than in others (Pollet, 2005).

From our empirical findings, two important implications can be derived. First, not all types of uncertainties matter for the complete cross-section of expected returns. The nature of the uncertainty, systematic or asset-specific and market relevant or industry relevant, determines whether it is a factor priced in equity returns. Uncertainty in the oil markets is an idiosyncratic and industry-specific risk factor that can be diversified away. This contrasts with the stock market uncertainty that reflects economic uncertainty and is by nature systematic. Second, our results are in accordance with the literature on the link between oil prices and the stock market. Oil prices influence the time series of stock returns (Driesprong et al., 2008), however, the relationship is more complex. Neither the complete cross-section of expected stock returns (Chen et al., 1986) nor the discount factors (Jones and Kaul, 1996) are affected. By looking at the specific effect of oil and gold price uncertainty, we find the existence of a sector-specific oil uncertainty factor, which provides evidence for the hypothesis of industry segmentation and specialization (Hong, Torous and Valkanov 2007).

\subsection{DATA AND METHODOLOGY}

Our data for the empirical analysis in the paper come from two different sources. We use S\&P 500 Index, West Texas Intermediate Crude Oil and Gold (100-oz) futures returns as our proxies for price changes in equity, oil and gold markets. We calculate the realized volatilities of the futures contracts written on these assets as estimations for their physical volatilities, and compute the volatilities implied by options traded on their future contracts as their risk-neutral volatilities. Hence to obtain prices of options and future contracts traded on each of these asset classes, we use the database of the Commodity Research Bureau (CRB). The CRB database, which has also been used by Doshi, Kumar and Yerramilli (2013) and Prokopczuk 


\section{CHAPTER 4}

and Simen (2014), provides us with various information on futures contracts and American put and call futures options. In particular, we obtain closing prices and transaction and expiration dates for the options and futures contracts traded on the S\&P 500 index, oil and gold.

The options are written on the futures contracts, hence, on each day, we match every option with its corresponding futures contract on the same day, and eliminate those for which we cannot find the underlying futures contract in the database. Also, due to illiquidity and microstructural anomalies, following Chang, Christoffersen and Jacobs (2013), we omit all options cheaper than $8 / 3$ dollars and options with less than six days to maturity. Table 4.1 displays some information about our data.

We investigate how investors' uncertainty about the equity, oil and gold markets affects the cross-section of stock returns. Thus we measure the volatility risk premium for a reasonably small time horizon. Prices of options with smaller time-to-maturity reflect investors' shortterm expectations and uncertainties more evidently. As Table 4.1 shows, the futures contracts on the S\&P 500 Index are written quarterly, which is less frequent compared to West Texas Intermediate Crude Oil and Gold (100-oz) futures; hence in order to have a unique and comparable horizon for our analysis, we take the smallest common time-to-maturity of 90 days for the volatility risk premium estimations $\left(\tau=\frac{1}{4}\right)$. As Table 4.1 shows, the number of observations in our database rises drastically over time, which implies considerably higher transaction volumes for these three different assets over the past years. Due to insufficient data for measuring oil volatility risk premium with $\tau=\frac{1}{4}$ in the earlier years, we conduct our analysis based on the last twenty years of data from 1996 to 2013.

In order to analyze the impact of market uncertainty on the cross-section of stock returns, we obtain the daily returns of all ordinary common shares traded at NYSE, AMEX and NASDAQ from the CRSP database. Furthermore, in order to calculate stock marketcapitalization at the end of each month, we download the prices and the number of shares outstanding for each of the stocks in the database.

As a measure for the uncertainty in each of the equity, oil and gold markets, we rely on each market's volatility risk premium, defined as the difference between expected physical and expected risk-neutral volatilities:

$V R P_{t}=E^{P}\left(\sigma_{t}^{\tau}\right)-E^{Q}\left(\sigma_{t}^{\tau}\right)$

where $\tau$ is the horizon we are looking at. On average, risk-neutral volatility is higher than physical volatility, therefore by buying a volatility swap contract and paying a volatility risk premium, investors can protect themselves against big shocks in volatility. In fact when investors' uncertainty escalates, the risk-neutral volatility increases and insurers will charge more for volatility swap contracts. Therefore the volatility risk premium provides a suitable representation of the market uncertainty. 
Table 4.1: Basic information about our database

\begin{tabular}{llll} 
& & Panel A: Futures & \\
\hline & S\&P 500 Index & Oil & Gold \\
\hline Exchange & CME & NYMEX & COMEX \\
First Date & $21 / 04 / 1982$ & $30 / 03 / 1988$ & $31 / 12 / 1974$ \\
Last Date & $31 / 12 / 2013$ & $31 / 12 / 2013$ & $31 / 12013$ \\
Trading Months & March, June, September, & Every Month & February, April, June, August, \\
& December & & October, December \\
\hline
\end{tabular}

Panel B: Options on Futures

\begin{tabular}{|c|c|c|c|c|c|c|c|c|c|c|}
\hline \multirow{2}{*}{ First Date } & \multicolumn{3}{|c|}{ S\&P 500 Index } & \multicolumn{4}{|c|}{ Oil } & \multicolumn{3}{|l|}{ Gold } \\
\hline & & \multicolumn{2}{|l|}{ 28/01/1983 } & \multicolumn{3}{|c|}{ 16/01/1989 } & \multicolumn{3}{|c|}{ 01/09/1988 } & \\
\hline \multirow{17}{*}{$\begin{array}{l}\text { Last Date } \\
\text { Observations } \\
\text { Cleaning }\end{array}$} & & \multicolumn{2}{|l|}{$31 / 12 / 2013$} & \multicolumn{3}{|c|}{$31 / 12 / 2013$} & \multicolumn{3}{|c|}{$31 / 12 / 2013$} & \\
\hline & Before & \multicolumn{2}{|l|}{$4,355,473$} & \multicolumn{3}{|c|}{$6,505,303$} & \multicolumn{3}{|c|}{$10,162,803$} & \\
\hline & Year & Total & Calls & Puts & Total & Calls & Puts & Total & Calls & Puts \\
\hline & 1983 & 6,440 & 3,405 & 3,035 & 0 & 0 & 0 & 0 & 0 & 0 \\
\hline & 1984 & 6,985 & 3,728 & 3,257 & 0 & 0 & 0 & 0 & 0 & 0 \\
\hline & 1985 & 8,621 & 4,388 & 4,233 & 0 & 0 & 0 & 0 & 0 & 0 \\
\hline & 1986 & 12,067 & 6,002 & 6,065 & 0 & 0 & 0 & 0 & 0 & 0 \\
\hline & 1987 & 19,165 & 9,696 & 9,469 & 0 & 0 & 0 & 0 & 0 & 0 \\
\hline & 1988 & 16,480 & 7,755 & 8,725 & 40 & 20 & 20 & 443 & 219 & 224 \\
\hline & 1989 & 17,771 & 8,905 & 8,866 & 11,552 & 5,595 & 5,957 & 19,156 & 10,601 & 8,555 \\
\hline & 1990 & 19,470 & 9,111 & 10,359 & 32,712 & 16,356 & 16,356 & 38,644 & 19,354 & 19,290 \\
\hline & 1991 & 20,845 & 9,483 & 11,362 & 38,766 & 21,004 & 17,762 & 37,787 & 18,961 & 18,826 \\
\hline & 1992 & 21,145 & 9,658 & 11,487 & 28,268 & 14,729 & 13,539 & 37,158 & 18,574 & 18,584 \\
\hline & 1993 & 22,549 & 10,274 & 12,275 & 32,775 & 17,824 & 14,951 & 56,946 & 28,478 & 28,468 \\
\hline & 1994 & 21,343 & 9,912 & 11,431 & 38,727 & 22,136 & 16,591 & 52,933 & 26,560 & 26,373 \\
\hline & 1995 & 36,409 & 18,155 & 18,254 & 46,492 & 28,088 & 18,404 & 55,221 & 27,613 & 27,608 \\
\hline & 1996 & 44,792 & 21,831 & 22,961 & 58,489 & 33,165 & 25,324 & 67,730 & 33,869 & 33,861 \\
\hline Observations & 1997 & 40,240 & 19,245 & 20,995 & 45,681 & 25,750 & 19,931 & 54,270 & 28,019 & 26,251 \\
\hline After & 1998 & 40,657 & 20,230 & 20,427 & 43,172 & 24,499 & 18,673 & 51,938 & 26,806 & 25,132 \\
\hline Cleaning & 1999 & 41,950 & 20,416 & 21,534 & 79,222 & 43,836 & 35,386 & 78,405 & 39,209 & 39,196 \\
\hline & 2000 & 72,786 & 33,720 & 39,066 & 141,773 & 71,291 & 70,482 & 100,119 & 50,033 & 50,086 \\
\hline & 2001 & 73,334 & 32,803 & 40,531 & 131,174 & 72,382 & 58,792 & 97,898 & 48,920 & 48,978 \\
\hline & 2002 & 77,613 & 36,832 & 40,781 & 140,740 & 77,902 & 62,838 & 114,001 & 57,011 & 56,990 \\
\hline & 2003 & 65,815 & 31,562 & 34,253 & 144,307 & 74,257 & 70,050 & 140,526 & 70,267 & 70,259 \\
\hline & 2004 & 68486 & 33,318 & 35,168 & 207,566 & 101,443 & 106,123 & 164,952 & 82,461 & 82,491 \\
\hline & 2005 & 76,055 & 36,020 & 40,035 & 352,751 & 171,342 & 181,409 & 186,782 & 93,403 & 93,379 \\
\hline & 2006 & 111,375 & 45,476 & 65,899 & 387,624 & 193,751 & 193,873 & 304,068 & 152,012 & 152,056 \\
\hline & 2007 & 150,453 & 55,582 & 94,871 & 419,028 & 216,838 & 202,190 & 291,847 & 145,954 & 145,893 \\
\hline & 2008 & 197,637 & 87,644 & 109,993 & 813,726 & 416,054 & 397,672 & 378,149 & 189,043 & 189,106 \\
\hline & 2009 & 174,061 & 80,224 & 93,837 & 783,286 & 406,853 & 376,433 & 458,061 & 229,073 & 228,988 \\
\hline & 2010 & 183,706 & 89,522 & 94,184 & 642,025 & 340,485 & 301,540 & 925,373 & 464,596 & 460,777 \\
\hline & 2011 & 309,518 & 155,511 & 154,007 & 675,634 & 354,199 & 321,435 & $1,386,915$ & 693,676 & 693,239 \\
\hline & 2012 & 337,200 & 168,511 & 168,689 & 700,938 & 371,861 & 329,077 & $1,657,902$ & 828,837 & 829,065 \\
\hline & 2013 & 363,152 & 181,649 & 181,503 & 507,227 & 270,695 & 236,532 & $1,692,184$ & 846,092 & 846,092 \\
\hline & Total & $2,658,120$ & $1,260,568$ & $1,397,552$ & $6,503,695$ & $3,392,355$ & $3,111,340$ & $8,449,408$ & $4,229,641$ & $4,219,767$ \\
\hline OTM Options & & & & & & & & & & \\
\hline $\begin{array}{l}\text { Used for } \\
\text { Calculating }\end{array}$ & Total & 409,977 & 229,431 & 180,546 & 314,152 & 208,467 & 105,685 & 420,993 & 285,715 & 135,278 \\
\hline $\begin{array}{l}\text { 90-Days Risk } \\
\text { Neutral } \\
\text { Volatility } \\
(1996-2013)\end{array}$ & $\begin{array}{l}\text { Average } \\
\text { Per Day }\end{array}$ & 90.46 & 50.62 & 39.84 & 69.61 & 46.19 & 23.42 & 93.35 & 63.35 & 30.00 \\
\hline
\end{tabular}




\section{CHAPTER 4}

Notes: This table provides some information about the futures contracts, and the futures options for the S\&P 500 Index, West Texas Intermediate Crude Oil and Gold (100-oz). We obtain this data from the Commodity Research Bureau database.

We calculate the annualized realized volatility of futures contracts for each asset class, on each day, as: ${ }^{4}$

$E^{P}\left(\sigma_{t}^{\tau}\right)=\sqrt{\frac{252}{360 \times \tau} \times \frac{1}{N-1} \times \sum_{s=t}^{t+360 \times \tau}\left(R_{s}^{\tau}-\overline{R^{\tau}}\right)^{2}}$

where $R_{S}^{\tau}=\left(\ln \left(F_{S}^{\tau}\right)-\ln \left(F_{S-1}^{\tau}\right)\right)$ is the logarithmic return of a futures contract with maturity $\tau$ at time $s$, and $\overline{R^{\tau}}$ represents the mean of all observed return values between $t$ and $t+\tau$. Futures with exactly 90 days to maturity $\left(\tau=\frac{1}{4}\right)$ are not necessarily being traded on every day. Therefore, in order to be able to calculate realized volatility with the constant horizon of $\tau=\frac{1}{4}$, on each day, if not available, we interpolate prices of closest futures contracts with shorter and longer maturities. We rely on the assumption that the ex-ante forecast of realized volatility is unbiased. This implies that the ex-post realized volatility is equal to the ex-ante forecast. This assumption is commonly used to compute the volatility risk premium (see e.g. Buraschi, Trojani and Vedolin, 2014). In the context of variance risk premium in commodities market, both Trolle and Schwartz (2010) and Prokopczuk and Simen (2013) rely on the expost realized variance to compute the variance risk premium.

Moreover, we use the Bakshi, Kapadia and Madan (2003) [BKM] model free methodology to calculate risk-neutral volatility time series. BKM methodology exploits the risk-neutral volatility of each day from out-of-money [OTM] European options traded on that specific day. Thus, the computed volatility is strictly conditional and forward-looking. BKM calculates risk-neutral volatility as:

$$
E^{Q}\left(\sigma_{t}^{\tau}\right)=\sqrt{\frac{e^{r \tau} V(t, t+\tau)-\mu(t, t+\tau)^{2}}{\tau}}
$$

where,

$\mu(t, t+\tau)=e^{r \tau}-1-\frac{e^{r \tau}}{2} V(t, \tau)-\frac{e^{r \tau}}{6} W(t, \tau)-\frac{e^{r \tau}}{24} X(t, \tau)$

\footnotetext{
${ }^{4}$ Some authors, such as Bali and Zhou (2014), proxy the realized variance with the second moment of the log returns, assuming $\overline{R^{\tau}}$ is zero in the long run. However as seasonality might deviate $\overline{R^{\tau}}$ from zero, to have more accurate estimation, we do not use the second moment.
} 


$$
\begin{aligned}
& V(t, t+\tau)=\int_{S(t)}^{\infty} \frac{2\left(1-\ln \left[\frac{K}{S(t)}\right]\right)}{K^{2}} C(t, t+\tau ; K) d K \\
& +\int_{0}^{S(t)} \frac{2\left(1+\ln \left[\frac{S(t)}{K}\right]\right)}{K^{2}} P(t, t+\tau ; K) d K \\
& W(t, \tau)=\int_{S(t)}^{\infty} \frac{6 \ln \left[\frac{K}{S(t)}\right]-3\left(\ln \left[\frac{K}{S(t)}\right]\right)^{2}}{K^{2}} C(t, t+\tau ; K) d K \\
& -\int_{0}^{S(t)} \frac{6 \ln \left[\frac{S(t)}{K}\right]+3\left(\ln \left[\frac{S(t)}{K}\right]\right)^{2}}{K^{2}} P(t, t+\tau ; K) d K
\end{aligned}
$$

and

$$
\begin{aligned}
X(t, \tau)= & \int_{S(t)}^{\infty} \frac{12\left(\ln \left[\frac{K}{S(t)}\right]\right)^{2}-4\left(\ln \left[\frac{K}{S(t)}\right]\right)^{3}}{K^{2}} C(t, t+\tau ; K) d K \\
& +\int_{0}^{S(t)} \frac{12\left(\ln \left[\frac{S(t)}{K}\right]\right)^{2}+4\left(\ln \left[\frac{s(t)}{K}\right]\right)^{3}}{K^{2}} P(t, t+\tau ; K) d K
\end{aligned}
$$

Here $K$ and $S$ are strike price and the underlying price, respectively. $C(t, t+\tau ; K)$ and $P(t, t+\tau ; K)$ respectively represent the price of a European call option and European put option at time $t$ with expiration date of $t+\tau$ and strike price of $K$.

Theoretically, the BKM methodology is only applicable for European options. However Bakshi, Kapadia and Madan (2003) argue that since the early-exercise premium of OTM options can be ignored, using American options does not change results meaningfully. Still, to be on the safe side, since all the options in our database are American type, we convert them to their European counterparts. To do this, following Trolle and Schwartz (2009), we adjust the prices by deducting early-exercise premia, measured according to the procedure outlined in Barone-Adesi and Whaley (1987).

To implement the BKM methodology, for each day we need a fine continuum of OTM European options with different strike prices. We consider the put options whose underlying price is more than 97 percent of strike price, and the call options whose underlying price is less than 103 percent of strike price, as OTM options. Also due to illiquidity, we eliminate put options with moneyness $\left(\frac{S(t)}{K}\right)$ value more than 1.5 and call options with moneyness $\left(\frac{S(t)}{K}\right)$ value less than 0.5 . The last two rows in Table 4.1 show the number of OTM options we used for calculating 90-day risk-neutral volatility. 


\section{CHAPTER 4}

Every day, only a few OTM call options and put options are being traded. Hence to be able to compute the integrals more accurately, we calculate the Black-Scholes implied volatility of each option and fit a natural cubic spline to them. ${ }^{5}$ Therefore we can determine implied volatilities and options prices, for every moneyness $\left(\frac{S(t)}{K}\right)$ from 0.01 to 2.01. Prices of OTM options with maturity of 90-day, and moneyness values outside this boundary, are negligible. In line with Chang, Christoffersen and Jacobs (2013), for options with higher moneyness than the maximum available moneyness and lower moneyness than the minimum available moneyness, we assume the implied volatility is constant and equal to the implied volatility of the highest moneyness and the lowest moneyness, respectively.

As with futures contracts, options with exactly 90 days to maturity $\left(\tau=\frac{1}{4}\right)$ are not necessarily being traded on every day. Therefore to calculate each day's risk-neutral volatility with constant horizon of $\tau=\frac{1}{4}$, on each day we calculate risk-neutral volatilities of the two closest maturities smaller and bigger than 90 days, and then interpolate between the computed volatilities to find an approximation of the 90 -day risk-neutral volatility.

\subsection{EMPIRICAL ANALYSIS}

\section{Descriptive Statistics}

Figure 4.1 plots the time series of the volatility risk premium of S\&P 500, oil and gold from 1996 to 2013. The premium is negative for the majority of the observations in all three markets. This indicates that volatility risk is priced. Investors are willing, on average, to lose money in order to hedge themselves against a change in volatility, not only of the S\&P equity portfolio but also of oil and gold.

The three time series exhibit substantial time variations. Significant correlation among the volatility risk premia of the S\&P 500 index, oil and gold reveals that some systematic patterns exist across the markets' volatility risk premia. Volatility swap sellers experienced a dramatic loss in 2008 on all three markets. This loss was most pronounced on the S\&P future market, and it was the least evident in the gold market. Moreover, the volatility risk premium increased in all three markets after the turmoil of 2008. This systematic surge in the spread between option implied volatility and realized volatility shows the increasing economic uncertainty at that time.

${ }^{5}$ If there are only two implied volatilities available, instead of fitting a cubic spline, we simply interpolate between them. 
Figure 4.1: Volatility risk premia of S\&P 500, oil and gold

\section{Panel (a): S\&P500 Volatility Risk Premium}

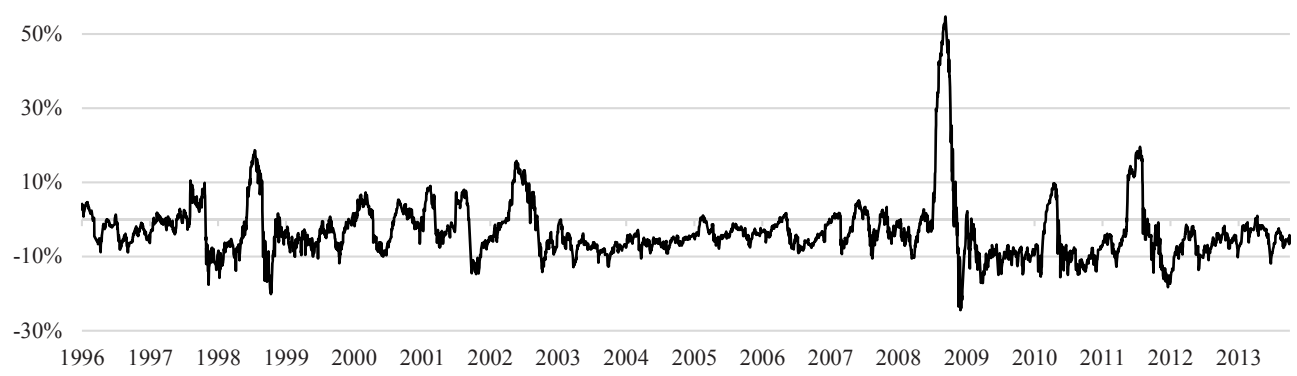

Panel (b): Oil Volatility Risk Premium

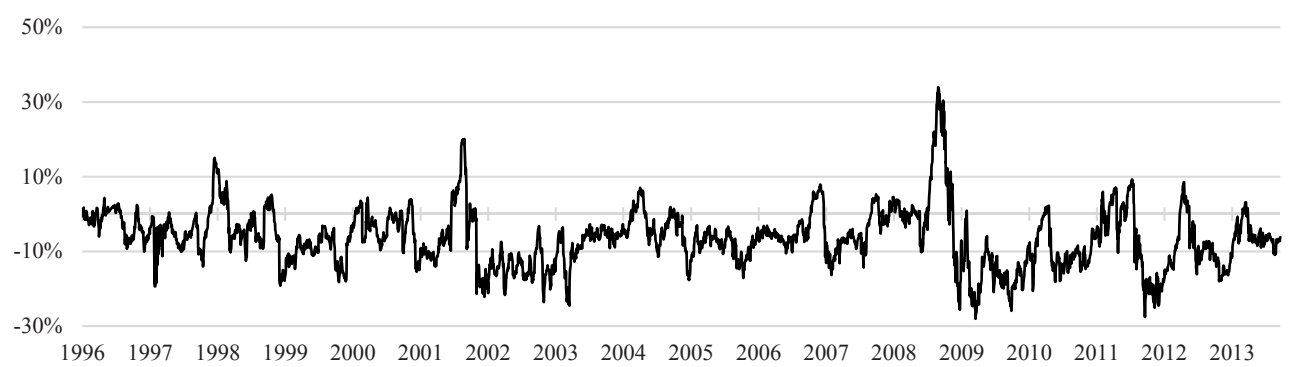

Panel (c): Gold Volatility Risk Premium

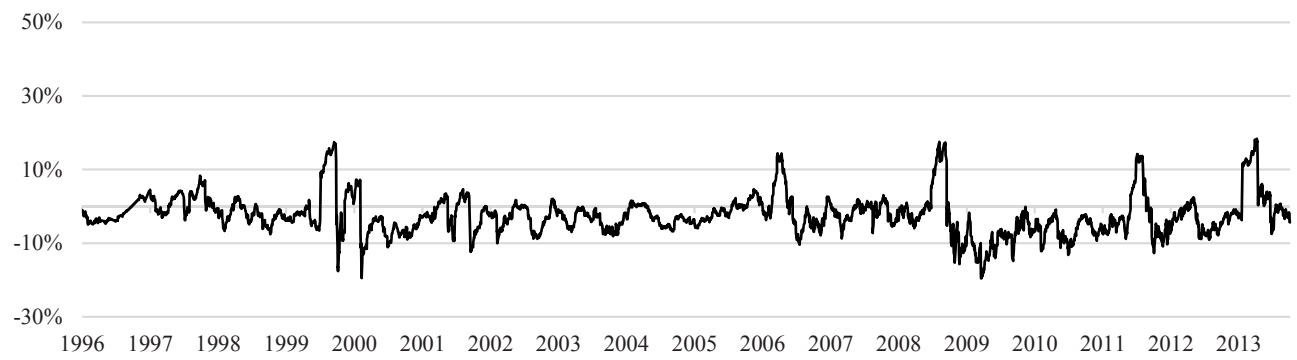

Note: We calculate volatility risk premium as the difference of realized volatility and option implied risk-neutral volatility of future contracts written on S\&P500 Index, Oil and Gold. 


\section{CHAPTER 4}

On the other hand, the three time series also exhibit some divergent movements. The contrasting picture from 2003 to 2008 pinpoints these differences. The S\&P volatility risk premium is very stable and steady during this period while both the crude oil and the gold volatility risk premia are more volatile. This shows the existence of an individual component in uncertainty. An even more compelling case follows from the surge of the oil volatility risk premium from the end of 2001 to mid-2003. During this period oil implied volatility surpassed oil realized volatility by $14.3 \%$ on average and even reached a peak of $24.5 \%$. The situation in the oil market during this period is well summarized by a quote from the New York Times on June 25, 2002.

"Yet in such unpredictable times, with one conflict worsening in the Middle East and the rumor of another rising, the 10-member cartel's inaction amounts to a gamble that could send the price of oil rocketing in the coming months." (Banerjee, 2002)

This episode illustrates the existence of market-specific uncertainty. This oil-specific component of market uncertainty motivates our investigation of the effect of market-specific uncertainty on equity markets.

Table 4.2 presents the descriptive statistics of the volatility risk premium. As suggested by the graphs, the average differences between realized volatility and implied volatility are negative and statistically significant for all three markets. In line with the results reported by Prokopczuk and Wese Simen (2013), gold has a relatively smaller volatility risk premium. The relatively high spread between crude oil options' implied volatility and crude oil realized volatility, consistent with the findings of Trolle and Schwartz (2010), is caused by the additional political uncertainty to which oil prices are exposed.

As previously stated, we are interested in the effect of news, the unexpected component of markets uncertainty. Accordingly, we use the volatility risk premia residuals from an $\operatorname{ARMA}(1,1)$ process. The significantly positive correlation between those residuals supports our previous graphical conjecture, that there is a systematic factor across all asset prices' uncertainty. However, those correlations are low and range from 15 percent between gold and S\&P to 24 percent between oil and S\&P. These correlations suggest the existence of two uncertainty components: a first systematic uncertainty component affecting all assets simultaneously, and a second asset-specific component that affects each asset individually. 
Table 4.2: Descriptive statistics on volatility risk premia

\begin{tabular}{llll}
\hline Statistics & \multicolumn{3}{c}{ Volatility Risk Premium } \\
\cline { 2 - 4 } & S\&P 500 & Oil & Gold \\
\hline Number of Observations & 4101 & 4395 & 4340 \\
Mean (\%) & -3.12 & -6.42 & -2.32 \\
Standard Deviation (\%) & $(-25.08)$ & $(-55.05)$ & $(-28.11)$ \\
\hline Percentiles & 7.97 & 7.74 & 5.43 \\
$5^{\text {th }}$ Percentile (\%) & & & -10.03 \\
$25^{\text {th }}$ Percentile (\%) & -12.25 & -18.51 & -5.56 \\
Median (\%) & -7.32 & -11.17 & -2.78 \\
$75^{\text {th }}$ Percentile (\%) & -4.49 & -6.53 & -0.09 \\
$95^{\text {th }}$ Percentile (\%) & -0.71 & -2.26 & 8.63 \\
\hline Correlations & 9.60 & 5.73 & \\
Oil & & & \\
Gold & 0.462 & 0.316 & \\
\hline Fitting ARMA (1, 1) & 0.451 & & 0.98 \\
AR Component & & 0.98 & -0.06 \\
MA Component & 0.99 & 0.13 & \\
Intercept & -0.09 & -0.02 & \\
\hline Innovations Correlation & -0.03 & & \\
Oil & 0.24 & & \\
Gold & 0.14 & & \\
\hline Notes:Wecan & & & \\
\hline
\end{tabular}

Notes:We calculate the volatility risk premium (VRP) as the difference of realized volatility and option implied risk-neutral volatility of future contracts written on S\&P500 Index, oil and gold. We take the residuals of fitted the ARMA $(1,1)$ model to VRP, as the innovations of the VRP process. The t-stats are shown in parentheses.

\section{Time Series Evidence}

In this section, we investigate the impact of uncertainty on the return dynamics of equities with time series regressions. As mentioned, the role of uncertainty from different markets is tested. This contrasts with the existing literature that focuses solely on stock market uncertainty (Anderson et al., 2009) or on economic uncertainty (Bekaert et al., 2009). Our decomposition highlights the interaction and spillover across markets at the uncertainty level, in addition to the return level. Moreover, we can quantify the relative importance of the alternative sources of uncertainty for equities and assess the impact of a common uncertainty factor across markets.

To acknowledge the heterogeneity among firm exposure to uncertainty, we test the relationships at the micro-firm level and not at the aggregated level. Unlike Driesprong et al. (2008), Narayan and Sharma (2011) also adopt a micro approach. They demonstrate that firms 


\section{CHAPTER 4}

are affected differently by oil price changes according to their industries and sizes. Accordingly, we perform the following basic contemporaneous time series regressions with daily observations on all stocks in the CRSP universe from 1996 to 2013.

\section{Model 1}

$R_{i t}=\alpha_{i}+\beta_{i} R_{m t}+\delta_{i}^{S \& P} V R P_{S \& P, t}+\gamma_{i}^{S \& P} R_{S \& P, t}+\varepsilon_{i t}$

$R_{i t}=\alpha_{i}+\beta_{i} R_{m t}+\delta_{i}^{O I L} V R P_{O I L, t}+\gamma_{i}^{O I L} R_{O I L, t}+\varepsilon_{i t}$

$R_{i t}=\alpha_{i}+\beta_{i} R_{m t}+\delta_{i}^{G O L D} V R P_{G O L D, t}+\gamma_{i}^{G O L D} R_{G O L D, t}+\varepsilon_{i t}$

Model 2

$R_{i t}=\alpha_{i}+\beta_{i} R_{m t}+\delta_{i}^{O I L} V R P_{O I L, t}+\delta_{i}^{S \& P} V R P_{S \& P, t} V R P_{S \& P, t}+\gamma_{i}^{O I L} R_{O I L, t}+\varepsilon_{i t}$

$R_{i t}=\alpha_{i}+\beta_{i} R_{m t}+\delta_{i}^{G O L D} V R P_{G O L D, t}+\delta_{i}^{S \& P} V R P_{S \& P, t}+\gamma_{i}^{G O L D} R_{G O L D, t}+\varepsilon_{i t}$

$R_{i t}$ is the excess return of stock $i$ at time $t, R_{m t}$ is the excess return of the market portfolio at time $t . V R P_{S \& P, t}, V R P_{O I L, t}$ and $V R P_{G O L D, t}$ are the innovations of the volatility risk premia of $\mathrm{S} \& \mathrm{P}$, oil and gold at time $t$. $R_{S \& P, t}, R_{O I L, t}$ and $R_{G O L D, t}$ are the returns of $\mathrm{S} \& \mathrm{P}$, oil and gold future contracts with 90 days to maturity at time $t$. Table 4.3 and 4.4 report the proportion of firms for which $\widehat{\delta}_{i}^{S \& P}, \hat{\delta}_{i}^{O I L}$ and $\hat{\delta}_{i}^{G O L D}$ are significantly positive, insignificantly positive, insignificantly negative and significantly negative for the two specifications. These statistics are summarized for the entire stock universe as well as industries (based on SIC code).

We perform a one-sided exact binomial test to check whether the number of firms significantly exposed to uncertainty is statistically different from zero. Significant results, at the $5 \%$ confidence level, are indicated in bold. Table 4.3 indicates that a substantial proportion of the $\widehat{\delta}_{i}^{S \& P}, \widehat{\delta}_{i}^{\text {OIL }}$ and $\widehat{\delta}_{i}^{\text {GOLD }}$ coefficients is significantly positive. This relationship is robust to the inclusion of the market, oil or gold futures returns, as displayed in Table 4.4. The distributions of the estimated coefficients are positively skewed. The number of positively significant $\widehat{\delta}_{\mathrm{i}}^{\mathrm{S} \& P}, \widehat{\delta}_{\mathrm{i}}^{\text {OIL }}$ and $\widehat{\delta}_{\mathrm{i}}^{\mathrm{GOLD}}$ coefficients are respectively $3.4,1.8$ and 2.8 times higher than the number of the same negatively significant coefficients. Only a small number of stocks offer a hedge against unpleasant change in uncertainty. We interpret these results as a strong indication that uncertainty matters for financial market. Equities are strongly affected by uncertainty, independently from risk. An increase in uncertainty is accompanied by a fall in equity prices. 
The Impact of Uncertainty in the Oil and Gold Market on the Cross-Section of Stock Returns

Table 4.3: Contemporaneous effect of uncertainty innovation on price of stocks

\begin{tabular}{|c|c|c|c|c|c|c|}
\hline \multirow{2}{*}{$\begin{array}{l}\text { Industry } \\
\text { Agriculture, Forestry and Fishing }\end{array}$} & \multicolumn{2}{|c|}{ S\&P 500 (1) } & \multicolumn{2}{|c|}{ Oil (2) } & \multicolumn{2}{|c|}{ Gold (3) } \\
\hline & & & & & & \\
\hline Number of Stocks & 43 & & 42 & & 42 & \\
\hline Positively Significant & 9 & $\% 20.9$ & 4 & $\% 9.5$ & 5 & $\% 11.9$ \\
\hline Positively Insignificant & 19 & $\% 44.2$ & 19 & $\% 45.2$ & 19 & $\% 45.2$ \\
\hline Negatively Insignificant & 12 & $\% 27.9$ & 16 & $\% 38.1$ & 15 & $\% 35.7$ \\
\hline Negatively significant & 3 & $\% 7.0$ & 3 & $\% 7.1$ & 3 & $\% 7.1$ \\
\hline \multicolumn{7}{|l|}{ Mining } \\
\hline Number of Stocks & 747 & & 752 & & 742 & \\
\hline Positively Significant & 225 & $\% 30.1$ & 162 & $\% 21.5$ & 134 & $\% 18.1$ \\
\hline Positively Insignificant & 336 & $\% 45.0$ & 343 & $\% 45.6$ & 323 & $\% 43.5$ \\
\hline Negatively Insignificant & 163 & $\% 21.8$ & 218 & $\% 29.0$ & 210 & $\% 28.3$ \\
\hline Negatively significant & 23 & $\% 3.1$ & 29 & $\% 3.9$ & 75 & $\% 10.1$ \\
\hline \multicolumn{7}{|l|}{ Construction } \\
\hline Number of Stocks & 155 & & 153 & & 153 & \\
\hline Positively Significant & 41 & $\% 26.5$ & 22 & $\% 14.4$ & 19 & $\% 12.4$ \\
\hline Positively Insignificant & 68 & $\% 43.9$ & 71 & $\% 46.4$ & 87 & $\% 56.9$ \\
\hline Negatively Insignificant & 46 & $\% 29.7$ & 51 & $\% 33.3$ & 40 & $\% 26.1$ \\
\hline Negatively significant & 0 & $\% 0.0$ & 9 & $\% 5.9$ & 7 & $\% 4.6$ \\
\hline \multicolumn{7}{|l|}{ Manufacturing } \\
\hline Number of Stocks & 4694 & & 4697 & & 4647 & \\
\hline Positively Significant & 997 & $\% 21.2$ & 492 & $\% 10.5$ & 644 & $\% 13.9$ \\
\hline Positively Insignificant & 2019 & $\% 43.0$ & 2201 & $\% 46.9$ & 2240 & $\% 48.2$ \\
\hline Negatively Insignificant & 1362 & $\% 29.0$ & 1711 & $\% 36.4$ & 1536 & $\% 33.1$ \\
\hline Negatively significant & 316 & $\% 6.7$ & 293 & $\% 6.2$ & 227 & $\% 4.9$ \\
\hline \multicolumn{7}{|c|}{ Transportation, Communications, Electric, Gas and Sanitary Service } \\
\hline Number of Stocks & 1184 & & 1184 & & 1167 & \\
\hline Positively Significant & 278 & $\% 23.5$ & 161 & $\% 13.6$ & 207 & $\% 17.7$ \\
\hline Positively Insignificant & 506 & $\% 42.7$ & 530 & $\% 44.8$ & 550 & $\% 47.1$ \\
\hline Negatively Insignificant & 343 & $\% 29.0$ & 427 & $\% 36.1$ & 365 & $\% 31.3$ \\
\hline Negatively significant & 57 & $\% 4.8$ & 66 & $\% 5.6$ & 45 & $\% 3.9$ \\
\hline \multicolumn{7}{|l|}{ Wholesale Trade } \\
\hline Number of Stocks & 602 & & 610 & & 603 & \\
\hline Positively Significant & 114 & $\% 18.9$ & 59 & $\% 9.7$ & 66 & $\% 10.9$ \\
\hline Positively Insignificant & 277 & $\% 46.0$ & 274 & $\% 44.9$ & 297 & $\% 49.3$ \\
\hline Negatively Insignificant & 176 & $\% 29.2$ & 238 & $\% 39.0$ & 214 & $\% 35.5$ \\
\hline Negatively significant & 35 & $\% 5.8$ & 39 & $\% 6.4$ & 26 & $\% 4.3$ \\
\hline
\end{tabular}




\section{CHAPTER 4}

Table 4.3 (Continued)

\begin{tabular}{|c|c|c|c|c|c|c|}
\hline \multirow{2}{*}{$\begin{array}{l}\text { Industry } \\
\text { Retail Trade }\end{array}$} & \multicolumn{2}{|c|}{ S\&P $500(1)$} & \multicolumn{2}{|l|}{ Oil (2) } & \multicolumn{2}{|l|}{ Gold (3) } \\
\hline & & & & & & \\
\hline Number of Stocks & 790 & & 788 & & 780 & \\
\hline Positively Significant & 139 & $\% 17.6$ & 58 & $\% 7.4$ & 70 & $\% 9.0$ \\
\hline Positively Insignificant & 348 & $\% 44.1$ & 335 & $\% 42.5$ & 363 & $\% 46.5$ \\
\hline Negatively Insignificant & 257 & $\% 32.5$ & 317 & $\% 40.2$ & 300 & $\% 38.5$ \\
\hline Negatively significant & 46 & $\% 5.8$ & 78 & $\% 9.9$ & 47 & $\% 6.0$ \\
\hline \multicolumn{7}{|c|}{ Finance, Insurance and Real Estate } \\
\hline Number of Stocks & 3372 & & 3369 & & 3334 & \\
\hline Positively Significant & 948 & $\% 28.1$ & 601 & $\% 17.8$ & 771 & $\% 23.1$ \\
\hline Positively Insignificant & 1385 & $\% 41.1$ & 1384 & $\% 41.1$ & 1413 & $\% 42.4$ \\
\hline Negatively Insignificant & 846 & $\% 25.1$ & 1096 & $\% 32.5$ & 920 & $\% 27.6$ \\
\hline Negatively significant & 193 & $\% 5.7$ & 288 & $\% 8.5$ & 230 & $\% 6.9$ \\
\hline \multicolumn{7}{|l|}{ Services } \\
\hline Number of Stocks & 2905 & & 2909 & & 2875 & \\
\hline Positively Significant & 375 & $\% 12.9$ & 257 & $\% 8.8$ & 327 & $\% 11.4$ \\
\hline Positively Insignificant & 1312 & $\% 45.2$ & 1301 & $\% 44.7$ & 1436 & $\% 49.9$ \\
\hline Negatively Insignificant & 965 & $\% 33.2$ & 1131 & $\% 38.9$ & 971 & $\% 33.8$ \\
\hline Negatively significant & 253 & $\% 8.7$ & 220 & $\% 7.6$ & 141 & $\% 4.9$ \\
\hline \multicolumn{7}{|l|}{ Public Administration } \\
\hline Number of Stocks & 14 & & 13 & & 13 & \\
\hline Positively Significant & 2 & $\% 14.3$ & 0 & $\% 0.0$ & 2 & $\% 15.4$ \\
\hline Positively Insignificant & 6 & $\% 42.9$ & 7 & $\% 53.8$ & 4 & $\% 30.8$ \\
\hline Negatively Insignificant & 5 & $\% 35.7$ & 6 & $\% 46.2$ & 6 & $\% 46.2$ \\
\hline Negatively significant & 1 & $\% 7.1$ & 0 & $\% 0.0$ & 1 & $\% 7.7$ \\
\hline \multicolumn{7}{|l|}{ Total } \\
\hline Number of Stocks & 14506 & & 14517 & & 14356 & \\
\hline Positively Significant & 3128 & $21,6 \%$ & 1816 & $12,5 \%$ & 2245 & $15,6 \%$ \\
\hline Positively Insignificant & 6276 & $43,3 \%$ & 6465 & $44,5 \%$ & 6732 & $46,9 \%$ \\
\hline Negatively Insignificant & 4175 & $28,8 \%$ & 5211 & $35,9 \%$ & 4577 & $31,9 \%$ \\
\hline Negatively significant & 927 & $6,4 \%$ & 1025 & $7,1 \%$ & 802 & $5,6 \%$ \\
\hline
\end{tabular}

Notes: For each sector we report the number of stocks which have significantly or insignificantly positive or negative exposure to the VRP innovations of our three different asset classes, namely S\&P 500, oil and gold, based on the regression equations:

$$
\begin{gathered}
\mathrm{R}_{\mathrm{it}}=\alpha_{\mathrm{i}}+\beta_{\mathrm{i}} \mathrm{R}_{\mathrm{mt}}+\delta_{\mathrm{i}}^{\mathrm{S \& P}} \mathrm{VRP}_{\mathrm{S \& P}, t}+\gamma_{\mathrm{i}}^{\mathrm{S \& P} P} \mathrm{R}_{\mathrm{S \& P}, t}+\varepsilon_{\mathrm{it}} \\
\mathrm{R}_{\mathrm{it}}=\alpha_{\mathrm{i}}+\beta_{\mathrm{i}} \mathrm{R}_{\mathrm{mt}}+\delta_{\mathrm{i}}^{\mathrm{OLL}} \mathrm{VRP}_{\mathrm{OLL}, \mathrm{t},}+\gamma_{\mathrm{i}}^{\mathrm{OLL}} \mathrm{R}_{\mathrm{OLL}, \mathrm{t}}+\varepsilon_{\mathrm{it}} \\
\mathrm{R}_{\mathrm{it}}=\alpha_{\mathrm{i}}+\beta_{\mathrm{i}} \mathrm{R}_{\mathrm{mt}}+\delta_{\mathrm{i}}^{\mathrm{GOLD}} \mathrm{VRP}_{\mathrm{GOLD}, \mathrm{t}}+\gamma_{\mathrm{i}}^{\mathrm{GOLD}} \mathrm{R}_{\mathrm{GOLD}, \mathrm{t}}+\varepsilon_{\mathrm{it}}
\end{gathered}
$$


$R_{i t}$ is the excess return of stock $i$ at time $t . R_{m t}$ is the excess return of the market portfolio at time $t$. $\mathrm{VRP}_{\mathrm{S} \& P, t}, \mathrm{VRP}_{\mathrm{OIL}, \mathrm{t}}$ and $\mathrm{VRP}_{\mathrm{GOLD}, \mathrm{t}}$ are the innovations of the volatility risk premia of $\mathrm{S} \& \mathrm{P}$, oil and gold at time $\mathrm{t}$. $\mathrm{R}_{\mathrm{S} \&, t}, \mathrm{R}_{\mathrm{OIL}, \mathrm{t}}$ and $\mathrm{R}_{\mathrm{GOLD}, \mathrm{t}}$ are the returns of $\mathrm{S} \& \mathrm{P}$, oil and gold future contracts with 90 days to maturity at time $\mathrm{t}$.

Table 4.4 shows that the $\widehat{\delta}_{\mathrm{i}}^{\text {OIL }}$ and $\widehat{\delta}_{\mathrm{i}}^{\mathrm{GOLD}}$ estimated coefficients are virtually unaffected by the inclusion of the S\&P volatility risk premium innovations in Model 2. The first specification, without controlling for the S\&P volatility risk premium innovation, yields $12.5 \%$ and $15.6 \%$ positively significant coefficients for $\widehat{\delta}_{\mathrm{i}}^{\mathrm{OIL}}$ and $\widehat{\delta}_{\mathrm{i}}^{\mathrm{GOLD}}$. After controlling for the S\&P volatility risk premium innovation, $10.8 \%$ and $14.5 \%$ of the $\hat{\delta}_{i}^{\text {OIL }}$ and $\hat{\delta}_{i}^{\text {GOLD }}$ coefficients remain positively significant. This robustness demonstrates that stocks returns are not only exposed to stock market uncertainty but also to oil and gold market uncertainty. Previous literature has documented the effect of oil prices on the equity market. We also find that the uncertainty on the oil and gold markets individually substantially affects equity prices. Therefore asset-specific uncertainties are also relevant for the equity market. This evidence pinpoints the importance both of the role of uncertainty in financial market and of the linkage across markets.

A comparison of the results for S\&P, oil and gold from Table 4.3 also shows that stock market uncertainty has a dominant impact on stock returns across the whole universe of firms. A greater proportion of stocks are significantly impacted by S\&P uncertainty compared to oil or gold uncertainty. On average, 21.6 percent of the stocks are exposed to S\&P uncertainty, while 12.5 percent and 15.6 percent of the firms are exposed to oil and gold uncertainty, respectively. S\&P uncertainty is also the dominant factor for every industry. This result is explained by the fact that the stock market reflects the information of the overall systematic economic outlook. In contrast, more specific forms of uncertainty such as political uncertainty or inflationary uncertainty influence uncertainties in the oil and gold market. Hence, only the firms subject to these specific sources of uncertainty are exposed to the oil and gold uncertainty factor.

Firms from all industries are consistently negatively affected by changes in uncertainty. But we observe significant variation across industries: the proportion of firms affected by uncertainty differs from one sector to another. For example, the 'Finance, Insurance and Real Estate' industry has $28.1 \%, 17.8 \%$ and $23.1 \%$ of its firms exposed negatively to S\&P, oil and gold uncertainty, whereas the 'Retail Trade' industry has as little as $17.6 \%, 7.4 \%$ and $9 \%$ of its firms exposed to the same factors. The dispersion in uncertainty sensitivity across industries highlights the need to account for the heterogeneity among different stocks and industries. 
Table 4.4: Controled contemporaneous effect of uncertainty on price of stocks

\begin{tabular}{|c|c|c|c|c|}
\hline \multirow{2}{*}{$\begin{array}{l}\text { Industry } \\
\text { Agriculture, Forestry and Fishing }\end{array}$} & \multicolumn{2}{|c|}{ Oil (1) } & \multicolumn{2}{|c|}{ Gold (2) } \\
\hline & & & & \\
\hline Number of Stocks & 42 & & 42 & \\
\hline Positively Significant & 2 & $\% 4.8$ & 5 & $\% 11.9$ \\
\hline Positively Insignificant & 18 & $\% 42.9$ & 20 & $\% 47.6$ \\
\hline Negatively Insignificant & 19 & $\% 45.2$ & 14 & $\% 33.3$ \\
\hline Negatively Significant & 3 & $\% 7.1$ & 3 & $\% 7.1$ \\
\hline \multicolumn{5}{|l|}{ Mining } \\
\hline Number of Stocks & 752 & & 742 & \\
\hline Positively Significant & 137 & $\% 18.2$ & 122 & $\% 16.4$ \\
\hline Positively Insignificant & 347 & $\% 46.1$ & 315 & $\% 42.5$ \\
\hline Negatively Insignificant & 236 & $\% 31.4$ & 218 & $\% 29.4$ \\
\hline Negatively Significant & 32 & $\% 4.3$ & 87 & $\% 11.7$ \\
\hline \multicolumn{5}{|l|}{ Construction } \\
\hline Number of Stocks & 153 & & 153 & \\
\hline Positively Significant & 16 & $\% 10.5$ & 19 & $\% 12.4$ \\
\hline Positively Insignificant & 71 & $\% 46.4$ & 82 & $\% 53.6$ \\
\hline Negatively Insignificant & 57 & $\% 37.3$ & 45 & $\% 29.4$ \\
\hline Negatively Significant & 9 & $\% 5.9$ & 7 & $\% 4.6$ \\
\hline \multicolumn{5}{|l|}{ Manufacturing } \\
\hline Number of Stocks & 4697 & & 4647 & \\
\hline Positively Significant & 407 & $\% 8.7$ & 594 & $\% 12.8$ \\
\hline Positively Insignificant & 2168 & $\% 46.2$ & 2243 & $\% 48.3$ \\
\hline Negatively Insignificant & 1825 & $\% 38.9$ & 1579 & $\% 34.0$ \\
\hline Negatively Significant & 297 & $\% 6.3$ & 231 & $\% 5.0$ \\
\hline
\end{tabular}

Transportation, Communications, Electric, Gas and Sanitary Service

\begin{tabular}{lllll}
\hline Number of Stocks & 1184 & & 1167 & \\
Positively Significant & 130 & $\% 11.0$ & 183 & $\% 15.7$ \\
Positively Insignificant & 533 & $\% 45.0$ & 565 & $\% 48.4$ \\
Negatively Insignificant & 449 & $\% 37.9$ & 372 & $\% 31.9$ \\
Negatively Significant & 72 & $\% 6.1$ & 47 & $\% 4.0$ \\
& & & & \\
Wholesale Trade & 610 & & 603 & \\
\hline Number of Stocks & 50 & $\% 8.2$ & 63 & $\% 10.4$ \\
Positively Significant & 266 & $\% 43.6$ & 292 & $\% 48.4$ \\
Positively Insignificant & 250 & $\% 41.0$ & 223 & $\% 37.0$ \\
Negatively Insignificant & 44 & $\% 7.2$ & 25 & $\% 4.1$ \\
Negatively Significant & & & & \\
& & & & \\
Retail Trade & 788 & & 590 & \\
\hline Number of Stocks & 49 & $\% 6.2$ & 370 & $\% 47.4$ \\
Positively Significant & 334 & $\% 42.4$ & & \\
Positively Insignificant & & & & \\
\end{tabular}


The Impact of Uncertainty in the Oil and Gold Market on the Cross-Section of Stock Returns

\begin{tabular}{|c|c|c|c|c|}
\hline Industry & Oil (1) & & Gold (2) & \\
\hline Negatively Insignificant & 326 & $\% 41.4$ & 303 & $\% 38.8$ \\
\hline Negatively Significant & 79 & $\% 10.0$ & 48 & $\% 6.2$ \\
\hline \multicolumn{5}{|c|}{ Finance, Insurance and Real Estate } \\
\hline Number of Stocks & 3369 & & 3334 & \\
\hline Positively Significant & 547 & $\% 16.2$ & 723 & $\% 21.7$ \\
\hline Positively Insignificant & 1352 & $\% 40.1$ & 1439 & $\% 43.2$ \\
\hline Negatively Insignificant & 1160 & $\% 34.4$ & 934 & $\% 28.0$ \\
\hline Negatively Significant & 310 & $\% 9.2$ & 238 & $\% 7.1$ \\
\hline \multicolumn{5}{|l|}{ Services } \\
\hline Number of Stocks & 2909 & & 2875 & \\
\hline Positively Significant & 235 & $\% 8.1$ & 308 & $\% 10.7$ \\
\hline Positively Insignificant & 1315 & $\% 45.2$ & 1438 & $\% 50.0$ \\
\hline Negatively Insignificant & 1140 & $\% 39.2$ & 995 & $\% 34.6$ \\
\hline Negatively Significant & 219 & $\% 7.5$ & 134 & $\% 4.7$ \\
\hline \multicolumn{5}{|l|}{ Public Administration } \\
\hline Number of Stocks & 13 & & 13 & \\
\hline Positively Significant & 0 & $\% 0.0$ & 1 & $\% 7.7$ \\
\hline Positively Insignificant & 6 & $\% 46.2$ & 5 & $\% 38.5$ \\
\hline Negatively Insignificant & 7 & $\% 53.8$ & 6 & $\% 46.2$ \\
\hline Negatively Significant & 0 & $\% 0.0$ & 1 & $\% 7.7$ \\
\hline \multicolumn{5}{|l|}{ Total } \\
\hline Number of Stocks & 14517 & & 14356 & \\
\hline Positively Significant & 1573 & $10,8 \%$ & 2077 & $14,5 \%$ \\
\hline Positively Insignificant & 6410 & $44,2 \%$ & 6769 & $47,2 \%$ \\
\hline Negatively Insignificant & 5469 & $37,7 \%$ & 4689 & $32,7 \%$ \\
\hline Negatively significant & 1065 & $7,3 \%$ & 821 & $5,7 \%$ \\
\hline
\end{tabular}

Notes: For each sector we report the number of stocks which have significantly or insignificantly positive or negative exposure to the VRP innovations of our three different asset classes, namely S\&P 500, oil and gold, based on the regression equations:

$$
\begin{aligned}
& \mathrm{R}_{\mathrm{it}}=\alpha_{\mathrm{i}}+\beta_{\mathrm{i}} \mathrm{R}_{\mathrm{mt}}+\delta_{\mathrm{i}}^{\text {S\&P }} \mathrm{VRP}_{\mathrm{S} \& P, t}+\delta_{\mathrm{i}}^{\mathrm{OLL}} \mathrm{VRP}_{\mathrm{OL}, \mathrm{t}}+\gamma_{\mathrm{i}}^{\mathrm{OLL}} \mathrm{R}_{\mathrm{OLL}, \mathrm{t}}+\varepsilon_{\mathrm{it}} \\
& \mathrm{R}_{\mathrm{it}}=\alpha_{\mathrm{i}}+\beta_{\mathrm{i}} \mathrm{R}_{\mathrm{mt}}+\delta_{\mathrm{i}}^{\mathrm{S} \& P} \mathrm{VRP}_{\mathrm{S} \& P, t}+\delta_{\mathrm{i}}^{\mathrm{GOLD}} \mathrm{VRP}_{\mathrm{GOLD}, \mathrm{t}}+\gamma_{\mathrm{i}}^{\mathrm{GOLD}} \mathrm{R}_{\mathrm{GOLD}, \mathrm{t}}+\varepsilon_{\mathrm{it}}
\end{aligned}
$$

$R_{i t}$ is the excess return of stock $i$ at time t. $R_{m t}$ is the excess return of the market portfolio at time $t . \mathrm{VRP}_{\mathrm{S} \& P, t}, \mathrm{VRP}_{\mathrm{OLL}, \mathrm{t}}$ and $\mathrm{VRP}_{\mathrm{GOLD}, \mathrm{t}}$ are the innovations of the volatility risk premia of $\mathrm{S} \& \mathrm{P}$, oil and gold at time t. $\mathrm{R}_{\mathrm{S} \&, t}, \mathrm{R}_{\mathrm{OIL}, \mathrm{t}}$ and $\mathrm{R}_{\mathrm{GOLD}, \mathrm{t}}$ are the returns of $\mathrm{S} \& \mathrm{P}$, oil and gold future contracts with 90 days to maturity at time $t$.

Some industries are clearly exposed to market-specific uncertainty. This is the case for the 'Mining' sector. According to the Model 2 specification, $\widehat{\delta}_{\mathrm{i}}^{\mathrm{OIL}}$ is positive and significant for $18.2 \%$ of the mining stocks. The upfront fixed costs necessary for oil extraction are important. Consequently, oil price unpredictability translates into profitability uncertainty for companies 


\section{CHAPTER 4}

mining this commodity. However, we observe that an industry highly exposed to a specific asset uncertainty is also more exposed to the other types of uncertainty. This feature characterizes the existence of a systematic uncertainty factor, as is also suggested by the positive correlations between volatility risk premia innovations.

\section{Cross-Sectional Evidence}

In the previous section, we demonstrated that uncertainty is negatively correlated with contemporaneous realized returns. Therefore uncertainty is relevant for equity valuation. Next, we turn our attention to testing whether our three sources of uncertainty also explain the cross-section of expected returns. Are equities compensated for their exposure to oil and gold uncertainty as well as to S\&P uncertainty?

The empirical link between uncertainty and expected return has been highlighted by Anderson et al. (2009) via a different measure. Bali and Zhou (2013), relying on a different methodology, use monthly observations and focus only on the S\&P 500 variance risk premium and the S\&P 500 firms cross-section. They show that portfolios more exposed to uncertainty are compensated by higher expected returns. An increase in uncertainty represents a bad outlook for uncertainty-averse agents. Consequently a premium is required for assets that correlate negatively with uncertainty.

In order to test whether stocks with different exposure to our three different types of uncertainty have different expected returns, we adopt the out-of-sample methodology of Harvey and Siddique (2000), Ang, Hodrick, Xing and Zhang (2006) and Chang, Christoffersen and Jacobs (2013). We measure the relative exposure of a stock to the S\&P, oil and gold uncertainty factors using the parameter estimates $\hat{\delta}_{i}^{S \& P}, \hat{\delta}_{i}^{O I L}$ and $\hat{\delta}_{i}^{G O L D}$ obtained from the regression (14), (15) and (16).

$$
\begin{aligned}
& R_{i t}=\alpha_{i}+\beta_{i} R_{m t}+\delta_{i}^{S \& P} V R P_{S \& P, t}+\varepsilon_{i t} \\
& R_{i t}=\alpha_{i}+\beta_{i} R_{m t}+\delta_{i}^{O I L} V R P_{O I L, t}+\varepsilon_{i t} \\
& R_{i t}=\alpha_{i}+\beta_{i} R_{m t}+\delta_{i}^{G O L D} V R P_{G O L D, t}+\varepsilon_{i t}
\end{aligned}
$$

To account for the time variation of the coefficient we use non-overlapping one month rolling window estimation on daily data. The same one month window interval is commonly used in the literature (Ang et al., 2006; Lewellen and Nagel, 2006; Chang et al., 2013) and offers a good balance between estimating the conditional factor loadings precisely and simultaneously allowing for time variation. 
We independently form five value-weighted portfolios sorted on each loading $\delta_{i}^{S \& P}, \delta_{i}^{O I L}$ and $\delta_{i}^{G O L D}$ ranging from low loading (P1) to high loading (P5). The procedure results in a total of 15 portfolios, five portfolios sorted on $\delta_{i}^{S \& P}$, five portfolios sorted on $\delta_{i}^{O I L}$ and five portfolios sorted on $\delta_{i}^{G O L D}$. In order to obtain sufficient cross-sectional dispersion in exposures across portfolios we use the entire CRSP universe and include all the NYSE, AMEX and NASDAQ ordinary common shares from 1996 to 2013. Stocks with missing data in one month are excluded from the analysis during this month. Portfolios are rebalanced based on the stock loadings at the end of every month and their performances are evaluated on the subsequent month. Table 4.5 reports the portfolio performance in terms of raw expected return, CAPM alpha, Fama and French (1993) three-factor alpha and Carhart (1997) four-factor alpha.

The portfolios sorted on $\hat{\delta}_{O I L}$ and $\hat{\delta}_{G O L D}$ do not display any specific pattern in terms of expected return. The same conclusion is reached after controlling for the classical market, SMB, HML and momentum risk factors. The lack of statistical significance in any of the performance measures for the high minus low portfolios corroborates this lack of relationship between the expected return and oil price uncertainty or gold price uncertainty. Therefore our results suggest that uncertainties in those markets are not market-wide priced risk factors.

The insignificance of the results obtained for the oil and gold market contrasts with the clear pattern obtained for the S\&P volatility risk premium. The portfolios sorted on $\hat{\delta}_{S \& P}$ display a monotonically increasing average return. The return difference between the portfolios P5 and $\mathrm{P} 1$ is equal to $0.71 \%$ on a monthly basis. This translates into an economically significant difference of $8.86 \%$ per year. This average return difference is also highly significant based on the Newey-West t-stat with 5 lags.

We evaluate the robustness of our results with respect to firm size. The stocks with extreme positive or extreme negative loadings on uncertainty factors are more likely to be small capitalization stocks. This implies that the P1 and P5 portfolios can be mainly composed of small stocks. To examine whether our previous results are confined within a subsample of the equity universe, we rely on a double sorting procedure. First we sort the stock universe in three size terciles. Then within each tercile we form five portfolios sorted on the previous uncertainty loading factors. The raw and risk-adjusted performances of the double-sorted portfolios are presented in Table 4.6.

As suspected, the absolute value of $\delta_{\mathrm{i}}^{\mathrm{S} \& P}, \delta_{\mathrm{i}}^{\mathrm{OIL}}$ and $\delta_{\mathrm{i}}^{\text {Gold }}$ for P1 to P5 is higher in the small size tercile and lower in the large size tercile. However, the previously obtained results are not concentrated within the small size tercile. The P5-P1 portfolios sorted on $\widehat{\delta}_{\text {OIL }}$ and $\widehat{\delta}_{\text {GOLD }}$ never provide statistically significant alphas at $5 \%$ confidence-level for any of the size groups, 


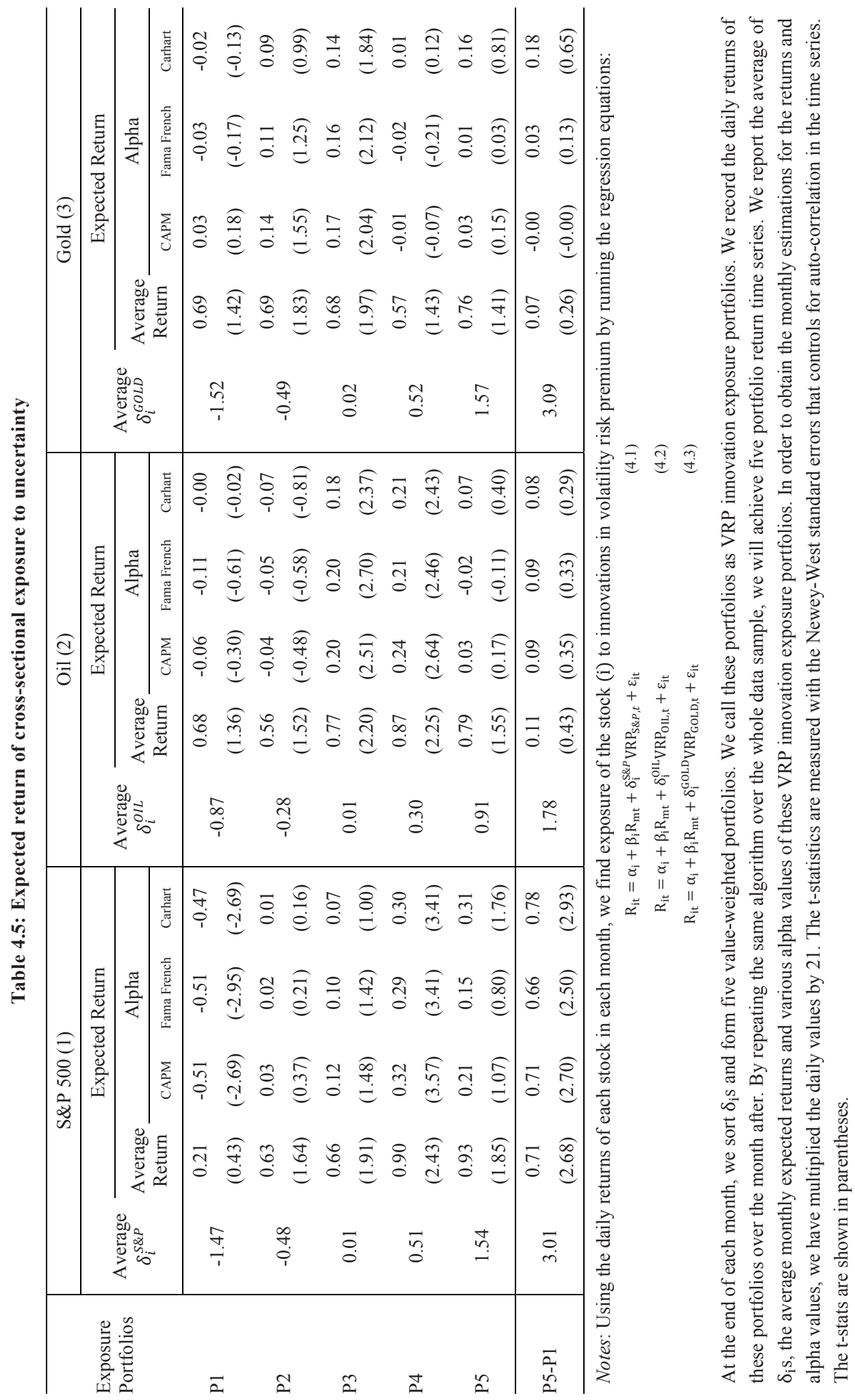


reconfirming that oil and gold price uncertainty are not priced factors. The significant premium found for exposure to S\&P uncertainty is not restricted to small stocks but is rather strong for large capitalisation firms. The sign of the S\&P uncertainty premium appears inverted for the smallest size tercile. This unexpected premium sign is explained by the lower reliability of the small size stocks results.

Also, the negative premium appears to be driven solely by the good performance of the P1 portfolio. The other portfolios' expected returns display a U-shaped and non-monotonic pattern. The significance of the negative premium amongst the smallest stocks is lower than the significance of the positive premium amongst other size terciles and even disappears for the Carhart four factors specification. Overall, our conclusion remains unchanged after controlling for size.

We find strong evidence that innovations in volatility risk premium or uncertainty of S\&P is a priced risk factor and explains the cross-section of expected return. This finding is consistent with theory and economic intuition. Stocks that experience a negative return when uncertainty increases are not a good hedge for uncertainty-averse investors. Accordingly, these stocks are compensated by higher expected returns. These results also confirm the findings of Bali and Zhou (2013). Our methodological approach diverges from this prior study in several ways. First, we use the whole stock universe and not solely the 500 biggest capitalisations. Second, we rely on past realized correlations to form our portfolios, while Bali and Zhou (2013) adopt a seemingly unrelated regression method together with a dynamic conditional covariance estimation to obtain the conditional exposures. Thirdly, unlike Bali and Zhou (2013) who use monthly observations, we run all our estimations with daily time series. Finally, we use 3 months S\&P futures option implied information instead of the one month VIX. Despite the differences in approach, the consistency of the findings as between the two studies reinforces confidence in the finding that uncertainty is a priced risk factor.

The main contribution of this paper is not to confirm that uncertainty is explaining the crosssection of expected return. However, we show that the nature of uncertainty matters regarding the expected return on equity. As empirically demonstrated, oil and gold price uncertainty contemporaneously negatively impacts an important proportion of equities. However, this linkage across markets does not exist at the expected return level. As illustrated in Figure 4.2, while S\&P uncertainty is priced in the cross-section of expected return, oil and gold uncertainty is not. We interpret this difference as evidence that oil and gold uncertainty factors are asset-specific, idiosyncratic and diversifiable. The S\&P 500, on the other hand, represents a systematic uncertainty factor that affects the overall economy and all the assets including the oil and gold market. We demonstrate that only the systematic part of uncertainty is relevant for the expected return of stocks. Recent work suggests that political uncertainty is related to stock market jumps (Baker, Bloom and Davis, 2013). Our results suggest that only the political uncertainty systematically related to economic uncertainty has an effect on expec- 


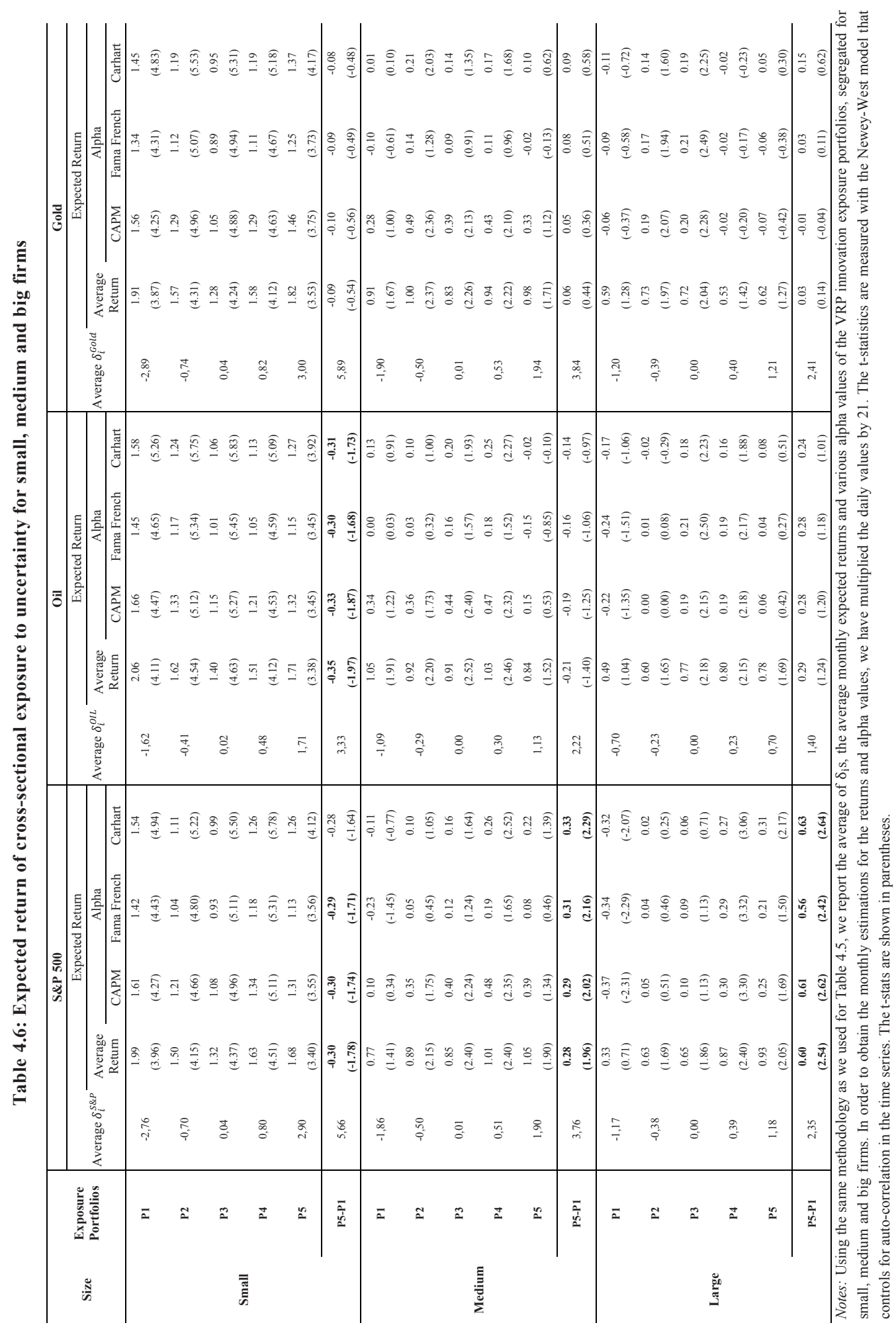


Figure 4.2: Expected return of cross-sectional exposure to uncertainty

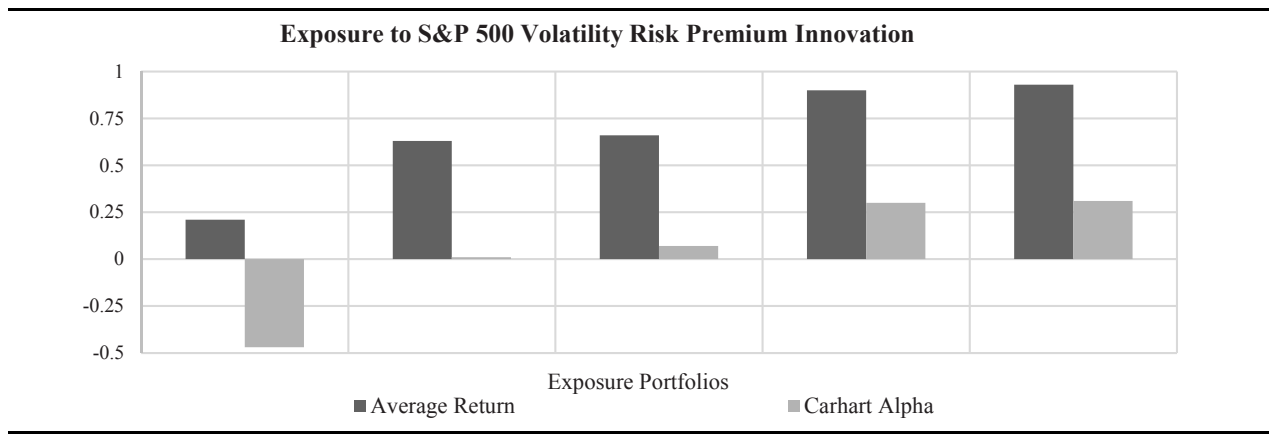

Exposure to Oil Volatility Risk Premium Innovation

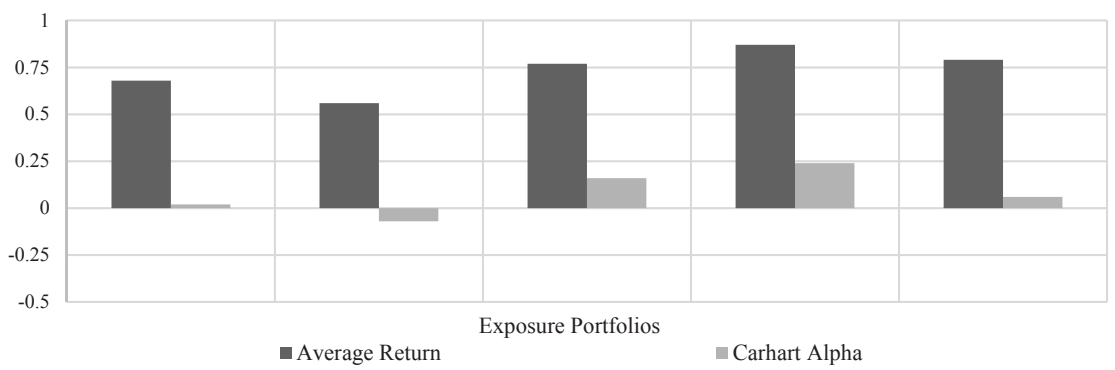

Exposure to Gold Volatility Risk Premium Innovation

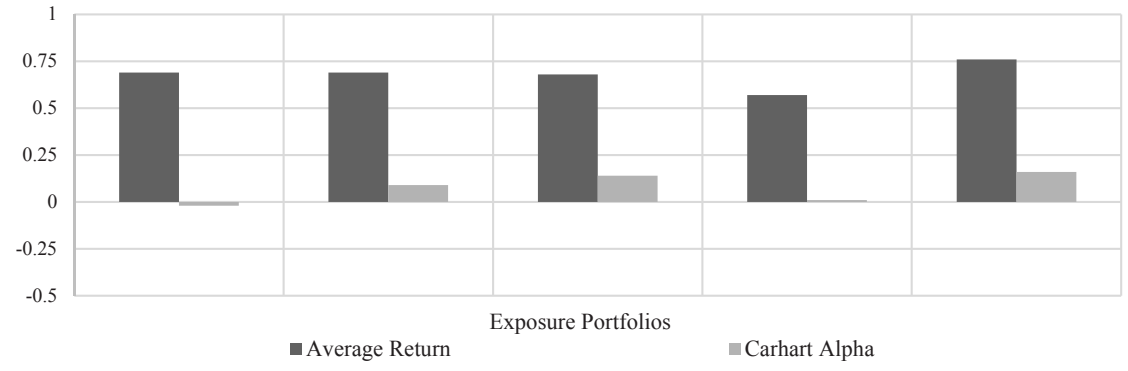

Notes: Using the daily returns of each stock in each month, we find exposure of the stock (i) to innovations in volatility risk premium by running regression equation:

$$
\begin{aligned}
& \mathrm{R}_{\mathrm{it}}=\alpha_{\mathrm{i}}+\beta_{\mathrm{i}} \mathrm{R}_{\mathrm{mt}}+\delta_{\mathrm{i}}^{\mathrm{S \& P} P} \mathrm{VRP}_{\mathrm{S} \& P, t}+\varepsilon_{\mathrm{it}} \\
& \mathrm{R}_{\mathrm{it}}=\alpha_{\mathrm{i}}+\beta_{\mathrm{i}} \mathrm{R}_{\mathrm{mt}}+\delta_{\mathrm{i}}^{\mathrm{OLL}} \mathrm{VRP}_{\text {OIL }, \mathrm{t}}+\varepsilon_{\mathrm{it}} \\
& \mathrm{R}_{\mathrm{it}}=\alpha_{\mathrm{i}}+\beta_{\mathrm{i}} \mathrm{R}_{\mathrm{mt}}+\delta_{\mathrm{i}}^{\mathrm{GOLD}} \mathrm{VRP}_{\mathrm{GOLD}, \mathrm{t}}+\varepsilon_{\mathrm{it}}
\end{aligned}
$$

At the end of each month, we sort the $\delta_{\mathrm{i}} \mathrm{s}$ and form five value-weighted portfolios. We refer to these portfolios as VRP innovation exposure portfolios. We record the daily returns of these portfolios over the month after. By repeating the same algorithm over the whole data sample, we achieve five portfolio return time series. We report the average of $\delta_{\mathrm{i}} \mathrm{s}$, the average monthly expected returns and various alpha values of these VRP innovation exposure portfolios. In order to obtain the monthly estimations for the returns and alpha values, we have multiplied the daily values by 21 . 


\section{CHAPTER 4}

ted stock returns. These results and the striking difference between the time series test and the cross-sectional test provide further support for the previous literature on the effect of oil prices on the equity market.

Although the oil price is found to affect stocks both at the aggregate level (Jones and Kaul, 1996; Driesprong et al., 2008) and at the micro-level (Narayan and Sharma, 2011), oil is often not found to be a priced risk factor (Chen et al., 1986; Ferson and Harvey, 1993) or to affect the discount rate (Jones and Kaul, 1996). We document empirically that this asymmetric effect exists not only for oil price but also for oil price uncertainty. These new results, together with previous findings, provide strong evidence that the oil price and also oil price uncertainty impact stock prices but not expected stock returns. The lack of relationship between oil and expected return also give support to the interpretation of Driesprong et al. (2008) that the oil-price-based return predictability is not explained by a time-varying premium. We conclude that oil market related information, although relevant for the overall economy, is not a systematic priced factor but rather an asset-specific factor.

\section{Further Evidence}

The above results demonstrate that unlike S\&P uncertainty, oil and gold price uncertainty are not market-wide priced factors. We now focus on the two latter sources of uncertainty and their effect on the equity market in the cross-section of expected return. Oil and gold price uncertainty are not found to affect the stock expected return, when tested on the entire universe of stocks and across business cycles. The nature of the relationship between the oil and gold markets and the equity market is complex and dynamic. It is widely recognized that investor attention is changing over time (Dallavigna and Pollet, 2009; Qian and Yu, 2009). A shift of attention toward these two markets would create the existence of a time-dependent oil and gold specific uncertainty premium. Veronesi (1999) and Qian and Yu (2009) provide evidence that investors' reaction to news depends on the state of the economy. Oil news is more informative about the economic outlook during recessions. Similarly, most of the attention paid to the gold market is concentrated during bad times, when this asset is seen as a safe haven (Baur and McDermott, 2010; Chan, Treepongkaruna, Brooks and Gray, 2011).

We test for the existence of time dependent oil and gold uncertainty premia with a subsample analysis. The sample is divided into two economic states: recession periods and expansion periods. As proxy for recession and expansion, we rely on the NBER business cycle indicator as in Henkel, Martin and Nardari (2011). NBER business cycles are not available to investors in real time, but they provide a good indication of the economic outlook for our exercise. Table 4.7 presents the results of the sub-sample analysis, divided between expansion and recession periods. 
As expected, returns are negative during recession periods and positive during expansion periods. The NBER business cycle indicators properly capture the long-term bullish and bearish trends in the US equity market. The outcome of the subsample analysis confirms the result obtained for the total sample. S\&P uncertainty is compensated in the cross-section of expected return in both recession and expansion periods. This systematic risk factor is priced at any time. However, neither in recessions nor during expansion is any pattern discernible for oil and gold. Oil and gold uncertainties are not priced in the cross-section of expected returns at any point in time. There is no evidence of time-varying linkage between oil and gold uncertainty and the equity market due to shifting attention.

Another important avenue to study is whether oil and gold uncertainty are sector-specific priced factors. The time series regressions show that oil and gold uncertainty are more relevant for certain industries. In contrast, stocks from every industry are exposed to S\&P uncertainty. Because of the asymmetric number of firms exposed significantly to the assetspecific uncertainty across industries, we test for the three uncertainty premia within each industry in Table 4.8. The shaded industries are the industries that are composed of less than 200 firms and therefore, cannot necessarily provide meaningful or interpretable results. Since there are fewer stocks in the cross-section of industries, we split the cross-sections into three value-weighted exposure portfolios.

Once again, our benchmark, the S\&P uncertainty, is priced in the cross-section of stocks. This more granular analysis shows that the price of uncertainty is positive in almost every industry and is significant in three industries. Thus uncertainty is priced across and within industries.

Although oil price uncertainty is not priced across industry, Table 4.8 reveals that we can find a positive compensation for bearing oil price uncertainty within three of the industries. In comparison with oil price uncertainty, gold price uncertainty is never priced in any industry. S\&P uncertainty is priced on the overall market. Oil price uncertainty is priced within certain specific sectors and gold price uncertainty is neither priced across nor within industries. This is the first evidence of oil price uncertainty being priced in the cross-section of stocks. The three industries where oil uncertainty is priced are 'Transportation, Communications, Electric, Gas and Sanitary Service', 'Wholesale Trade' and 'Finance, Insurance and Real Estate'. The two first sectors are industries for which oil price is an important economic input of the core activity. This relevance is statistically highlighted by the time series regressions that showed that these sectors are characterized by a higher proportion of stocks significantly exposed to the oil uncertainty factor. Therefore, oil uncertainty is priced within oil dependent industries. There are two explanations as to why oil uncertainty is only significant at the industry level. The first explanation is related to econometric factors. In a cross-sectional test, a sufficient dispersion among the different observations with respect to a factor, exposure to uncertainty in our case, is necessary in order to detect any significant risk premium. A risk factor can be priced but not statistically identifiable if all assets are almost equally exposed to this specific factor. 


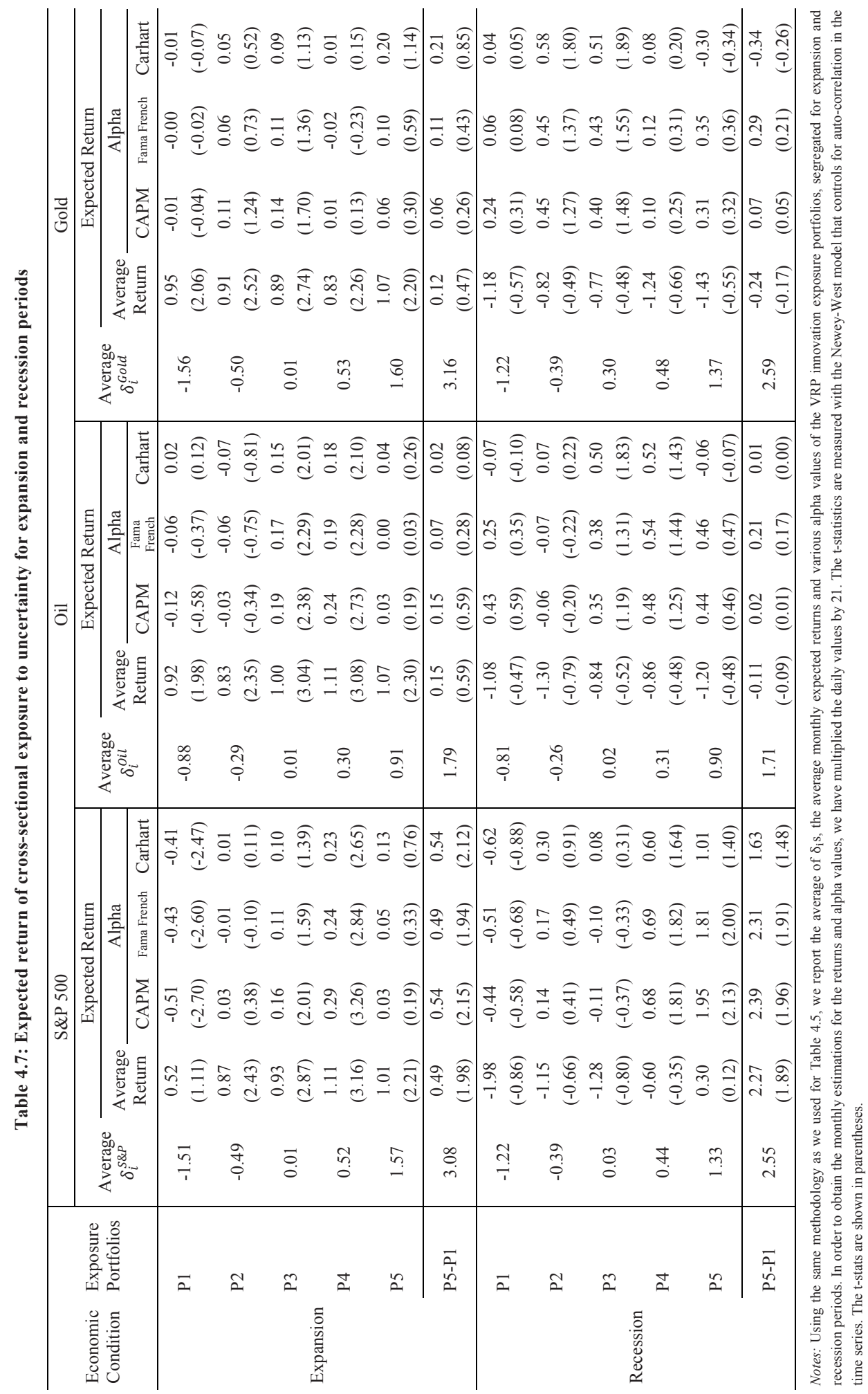


The Impact of Uncertainty in the Oil and Gold Market on the Cross-Section of Stock Returns

Table 4.8: Expected return of cross-sectional exposure to uncertainty for different industries

\begin{tabular}{|c|c|c|c|c|c|c|c|}
\hline \multirow[t]{2}{*}{ Industry } & \multirow[b]{2}{*}{ Average Return } & \multicolumn{2}{|c|}{ S\&P 500} & \multicolumn{2}{|l|}{ Oil } & \multicolumn{2}{|l|}{ Gold } \\
\hline & & -0.11 & $(-0.15)$ & 0.15 & $(0.23)$ & 0.47 & $(0.73)$ \\
\hline \multirow{4}{*}{$\begin{array}{l}\text { Agriculture, Forestry } \\
\text { and Fishing }\end{array}$} & CAPM Alpha & -0.11 & $(-0.16)$ & 0.18 & $(0.29)$ & 0.44 & $(0.68)$ \\
\hline & Fama French Alpha & -0.11 & $(-0.16)$ & 0.22 & $(0.35)$ & 0.48 & $(0.75)$ \\
\hline & Carhart Alpha & -0.14 & $(-0.20)$ & 0.20 & $(0.32)$ & 0.54 & $(0.84)$ \\
\hline & Average Return & 0.25 & $(0.74)$ & 0.29 & $(0.77)$ & -0.19 & $(-0.52)$ \\
\hline \multirow{3}{*}{ Mining } & CAPM Alpha & 0.22 & $(0.64)$ & 0.30 & $(0.76)$ & -0.28 & $(-0.77)$ \\
\hline & Fama French Alpha & 0.19 & $(0.56)$ & 0.28 & $(0.72)$ & -0.30 & $(-0.83)$ \\
\hline & Carhart Alpha & 0.29 & $(0.83)$ & 0.24 & $(0.63)$ & -0.25 & $(-0.66)$ \\
\hline \multirow{4}{*}{ Construction } & Average Return & 0.71 & $(1.52)$ & 1.44 & $(3.09)$ & 1.33 & $(2.76)$ \\
\hline & CAPM Alpha & 0.75 & $(1.61)$ & 1.45 & $(3.10)$ & 1.34 & $(2.78)$ \\
\hline & Fama French Alpha & 0.73 & $(1.57)$ & 1.37 & $(2.96)$ & 1.30 & $(2.72)$ \\
\hline & Carhart Alpha & 0.69 & $(1.48)$ & 1.45 & $(3.14)$ & 1.39 & $(2.91)$ \\
\hline \multirow{4}{*}{ Manufacturing } & Average Return & 0.51 & $(2.00)$ & 0.24 & $(0.93)$ & -0.12 & $(-0.45)$ \\
\hline & CAPM Alpha & 0.52 & $(2.03)$ & 0.22 & $(0.83)$ & -0.17 & $(-0.64)$ \\
\hline & Fama French Alpha & 0.50 & $(1.98)$ & 0.19 & $(0.73)$ & -0.13 & $(-0.50)$ \\
\hline & Carhart Alpha & 0.57 & $(2.22)$ & 0.15 & $(0.58)$ & -0.00 & $(-0.00)$ \\
\hline \multirow{4}{*}{$\begin{array}{l}\text { Transportation, } \\
\text { Communications, } \\
\text { Electric, Gas and } \\
\text { Sanitary service }\end{array}$} & Average Return & -0.01 & $(-0.04)$ & 0.63 & $(2.10)$ & -0.08 & $(-0.29)$ \\
\hline & CAPM Alpha & -0.02 & $(-0.09)$ & 0.60 & $(2.01)$ & -0.12 & $(-0.44)$ \\
\hline & Fama French Alpha & -0.05 & $(-0.18)$ & 0.58 & $(1.93)$ & -0.12 & $(-0.42)$ \\
\hline & Carhart Alpha & -0.02 & $(-0.06)$ & 0.61 & $(2.00)$ & -0.08 & $(-0.29)$ \\
\hline \multirow{4}{*}{ Wholesale Trade } & Average Return & 0.33 & $(1.00)$ & 0.61 & $(1.84)$ & -0.08 & $(-0.22)$ \\
\hline & CAPM Alpha & 0.36 & $(1.06)$ & 0.61 & $(1.84)$ & -0.11 & $(-0.30)$ \\
\hline & Fama French Alpha & 0.34 & $(1.02)$ & 0.61 & $(1.85)$ & -0.10 & $(-0.28)$ \\
\hline & Carhart Alpha & 0.42 & $(1.23)$ & 0.63 & $(1.90)$ & 0.00 & $(0.01)$ \\
\hline \multirow{4}{*}{ Retail Trade } & Average Return & 0.09 & $(0.32)$ & -0.30 & $(-1.07)$ & -0.46 & $(-1.59)$ \\
\hline & CAPM Alpha & 0.09 & $(0.30)$ & -0.32 & $(-1.15)$ & -0.45 & $(-1.58)$ \\
\hline & Fama French Alpha & 0.09 & $(0.31)$ & -0.35 & $(-1.26)$ & -0.44 & $(-1.54)$ \\
\hline & Carhart Alpha & 0.14 & $(0.47)$ & -0.35 & $(-1.26)$ & -0.46 & $(-1.58)$ \\
\hline \multirow{4}{*}{$\begin{array}{l}\text { Finance, Insurance and } \\
\text { Real Estate }\end{array}$} & Average Return & 0.35 & $(1.66)$ & 0.45 & $(2.09)$ & -0.10 & $(-0.47)$ \\
\hline & CAPM Alpha & 0.38 & $(1.84)$ & 0.43 & $(2.00)$ & -0.13 & $(-0.60)$ \\
\hline & Fama French Alpha & 0.36 & $(1.77)$ & 0.46 & $(2.10)$ & -0.07 & $(-0.33)$ \\
\hline & Carhart Alpha & 0.36 & $(1.73)$ & 0.46 & $(2.12)$ & -0.05 & $(-0.24)$ \\
\hline \multirow{4}{*}{ Services } & Average Return & 0.63 & $(2.31)$ & -0.08 & $(-0.27)$ & 0.26 & $(0.92)$ \\
\hline & CAPM Alpha & 0.64 & $(2.32)$ & -0.09 & $(-0.29)$ & 0.22 & $(0.79)$ \\
\hline & Fama French Alpha & 0.61 & $(2.19)$ & -0.10 & $(-0.33)$ & 0.25 & $(0.87)$ \\
\hline & Carhart Alpha & 0.63 & $(2.27)$ & -0.05 & $(-0.17)$ & 0.31 & (1.09) \\
\hline \multirow{4}{*}{ Public Administration } & Average Return & 2.30 & $(1.23)$ & 1.84 & $(0.97)$ & 2.74 & $(1.48)$ \\
\hline & CAPM Alpha & 2.34 & $(1.24)$ & 1.78 & $(0.93)$ & 2.68 & $(1.45)$ \\
\hline & Fama French Alpha & 2.43 & $(1.28)$ & 1.76 & $(0.93)$ & 2.57 & $(1.41)$ \\
\hline & Carhart Alpha & 2.25 & $(1.19)$ & 1.87 & $(0.98)$ & 2.77 & $(1.48)$ \\
\hline
\end{tabular}




\section{CHAPTER 4}

Notes: We split the cross-section of each industry into three different exposure levels. Then we report the average monthly expected returns and various alpha values of the high minus low VRP innovation exposure portfolio. In order to obtain the monthly estimations for the returns and alpha values, we have multiplied the daily values by 21 . The t-statistics are measured with the Newey-West corrections that control for autocorrelation in the time series. The t-stats are shown in parentheses.

For instance, as Borgers, Derwall, Koedijk and Ter Horst (2014) show, the sin stock premium can only be detected for sin stock funds and not standard mutual funds because the latter funds are homogeneous with respect to their 'sin exposure'. In our study, certain industries are characterized by very little exposure to oil uncertainty.

The majority of the stocks are homogeneously not exposed to this factor and thereby no premium can be detected. This interpretation suggests that there is an oil-specific uncertainty premium; nevertheless so few stocks are exposed significantly to it that it is hard to detect. Although certain sectors are relatively more exposed to gold price uncertainty, no gold price uncertainty premium is detected within any industry. This result highlights a fundamental difference between oil and gold price uncertainty.

The other, more economic, reason relates to the segmentation of markets. Numerous academic papers have investigated geographic segmentation. Different investors invest in different places. Accordingly, risk is priced differently across the globe (Heston, Rouwenhorst and Wessels, 1995; Hou, Karolyi and Kho, 2011). Cavaglia, Brightman and Aked (2000) find that global diversification has decreased as the market became more integrated, while industry diversification has increased. Hong, Torous and Valkanov (2007) claim that a considerable proportion of investors are industry specialized, causing industries segmentation. Menzy and Ozbas (2010) and Cohen and Frazzini (2008) show that different news is reflected more or less quickly and accurately in different industries. Hong and Stein (2007) interpret this finding as follows, "Thus, information appears to flow gradually across industries, perhaps because each industry has its own set of specialist investors who focus on uncovering the most directly industry-relevant information, and who only slowly become aware of events in related industries. '(Hong and Stein, 2007:118).

A similar interpretation of our results lends credence to the explanation that investors in an oil relevant industry are more aware of the impact of oil on their investment. Accordingly, oil uncertainty is timely priced amongst these firms compared to the firms in other sectors. Driesprong et al. (2008) found that oil price information was incorporated slowly in equity prices. For certain industries, oil relevant information is incorporated faster in equity prices (Narayan and Sharma, 2011). Pollet (2005) shows that the impact of predictable oil price changes is misevaluated and incorporated slowly for non-oil relevant industries. Similarly, the impact of oil uncertainty can only be evaluated properly for the oil relevant industries and cannot be incorporated in the expected return of other industries' firms. This reasoning also explains the absence of a gold price uncertainty premium. Stocks are exposed to gold 
uncertainty because it captures some variations in the macro-economic environment. However, very few firms are directly affected by changes in the price of gold apart from corporations involved in the actual trading of gold. In contrast, the benefits, the profitability and the costs of numerous firms are substantially affected by oil prices. Additionally, investors specialized and concentrated in oil relevant stocks cannot diversify the oil uncertainty risk across their portfolio. For these types of investors, oil uncertainty directly affects their marginal utility and they cause oil uncertainty to be a priced factor within certain industries.

Although the two explanations provided are different, they point to the same conclusion. Oil uncertainty is not a globally priced factor. Either, as has become the case for liquidity exposure among large stocks (Ben-Rephael, Kadan and Wohl, 2008), this is because too few stocks are actually exposed to this risk factor. Or it is because of the segmentation of industries, leading to a risk factor only to be compensated for oil relevant industries. Finally, we find evidence of oil uncertainty being priced, but this premium is less relevant for the entire universe of stocks than S\&P uncertainty.

\subsection{CONCLUSION}

In this chapter, we evaluated the impact of oil and gold uncertainty on the equity market and compared it with the impact of S\&P uncertainty on the equity market. We segregated stock market uncertainty, oil price uncertainty and gold price uncertainty. In order to obtain a coherent measure of uncertainty for each asset, we relied on the volatility risk premium extracted from S\&P, oil and gold options. The volatility risk premia time series share a common component attributable to a systematic uncertainty factor affecting all asset prices. However, we also find clear evidence of asset-specific uncertainty.

Stock returns are severely affected by all three types of uncertainty. Measuring oil and gold uncertainty independently allows us to show that market-specific uncertainty also negatively affects a considerable proportion of firms, especially within certain industries. Therefore, not only oil price but also oil price uncertainty matters for stock returns. This shows that the stability of the oil market and the uncertainty around OPEC policy is a source of vulnerability for the stock market.

Although oil and gold price uncertainty negatively influence stock returns, exposure to those factors is not compensated in the stock market. On the contrary, S\&P uncertainty is a priced factor. This important result reveals that the compensation for uncertainty depends on the nature of uncertainty. Only systematic uncertainty is priced, while asset-specific uncertainty is not. This result is robust across business cycles. 


\section{CHAPTER 4}

Moreover, we interpret our results as additional evidence that oil market information is relevant only for the time series of stock return but not the cross-sectional of the entire expected stock return as has previously been demonstrated for oil returns. Oil uncertainty is non-systematic and more industry-specific. This is why the oil uncertainty premium is not relevant for every stock, but only for the stocks within oil relevant market segmented industries. 


\section{Chapter 5}

\section{Forecasting Volatility or Value-at-Risk: when does the Volatility Risk Premium matter? ${ }^{6}$}

\subsection{INTRODUCTION}

Numerous empirical works demonstrate the superiority of option implied volatility over historical volatility model in predicting future asset volatility. We contribute to this literature, evaluating and comparing the merits of implied volatility (IV) and historical volatility models for Value-at-Risk (VaR) forecasting. Volatility forecasting and VaR forecasting are two different objectives. Hence this chapter extends the comparison between IV and time series information into another field. Furthermore, we show that the best volatility forecast does not coincide with the best VaR forecast. The results of our multiple and complementary backtesting procedures show that IV based VaR cannot outperform the standard historical volatility based VaR model.

The volatility risk premium embedded in option prices explains the disappointing performance of IV for VaR forecasting. Simple volatility risk premium adjustments are sufficient to transform IV into an appropriate volatility forecast. On the contrary, simple corrections applied in this paper do not allow IV based VaR to outperform historical volatility model VaR.

Despite the earlier studies indicating the poor information content of IV (Canina and Figlewski, 1993), the great majority of the volatility prediction literature concludes that IV either totally subsumes historical volatility model forecasts (Fleming, 1998;Christensen and Prabhala, 1998; Blair, Poon and Taylor, 2001) or at least complements it (Beckers, 1981; Day and Lewis, 1992). This conclusion has been reached on different markets including equity indices (Corrado and Miller, 2005; Yu, Lui and Wang, 2010), individual equities (Taylor, Yadav and Zhang, 2010), currencies (Charoenwong, Jenwittayarohe and Sin Low, 2009) and commodities (Szakmary, Ors, Kim and Davidson, 2003). Only intraday times series volatility information appears to compete with IV information (Taylor and Xu, 1997; Pong, Shackelton, Taylor and $\mathrm{Xu}, 2004)$.

${ }^{6}$ This chapter is co-authored with Dennis Bams (Maastricht University) and Thorsten Lehnert (Luxembourg University) 


\section{CHAPTER 5}

Many of the studies in this field justify their investigations in the name of risk management (Martens and Zein, 2004; Frijns, Tallau and Tourani-Rad, 2012). But surprisingly, almost no study has actually evaluated the performance of IV versus historical volatility models in a risk management application. This chapter evaluates whether the IV superior volatility forecasting power is translated into superior VaR prediction compared to historical volatility models. The empirical design adopted here differs from Giot (2005) which is to our knowledge the only other study dedicated to the use of IV in a VaR forecasting exercise. We rely on a much longer time series of data and do not include IV in the conditional volatility equation. First, this allows for a clear distinction on the relative benefits of standalone IV. Second, we can account formally for the volatility risk premium affecting the IV forecasts.

IV based forecasts, although efficient, are documented as being biased (Lamoureux and Lastrapes, 1993; Szakmary et al., 2003; Yu et al., 2010). Chernov (2007) formally identifies the origin of this bias, namely the volatility risk premium. Volatility risk premium is the difference in expected volatility between the physical measure and the risk-neutral measure. The price of volatility risk being negative, the risk neutral expectation of volatility implied from option prices is higher than the physical expectation of volatility, which is relevant for the VaR calculation. This feature affects the predictive power of IV negatively (Tsiaras, 2009). Prokopczuk and Wese Simen (2014) are the first to formally acknowledge and account for the variance risk premium in an assessment of the forecasting performance of IV. They demonstrate empirically that a simple adjustment or correction of the implied volatilities for the variance risk premium improves the forecasting performance over the standard IV both in and out-of-sample.

We account for the volatility risk premium in the same spirit as Prokopczuk and Wese Simen (2014). However, we apply this procedure in the VaR prediction context instead. Moreover, we focus on three major equity indices with publically available implied volatility indices rather than the commodity market that requires a proprietary option dataset. The dynamics of the IV and the volatility risk premium diverge across markets and assets (Martin, Reidy and Wright, 2009). Hence, it is relevant to evaluate the performance of adjusted IV forecasts on more mainstream markets such as major equity indices.

We assess the information content of historical volatility models, IV and IV adjusted for the volatility risk premium over an extended time period for the purpose of VaR measurement. We adjust for the volatility risk premium bias both non-parametrically and parametrically and compare the obtained results. We measure the VaR for three major stock indices, the S\&P 500, DJIA and NASDAQ 100, using three implied volatility indices SPX (1990-2013), VXD (1997-2013) and VXN (2001-2013). We study 1\% and 5\% level VaR as well as 1 day out of sample and 1 month out of sample VaR. Although not used in practice, the one month VaR is also computed in this study in order to match the maturity of the implied volatility indices. 
We evaluate the VaR models relying on a set of three complementary tests: the LR test of Kupiec (1995), the dynamic quantile test of Engle and Manganelli (2004) and a VaR specific loss function. We bootstrap this loss function as in Chen and Gerlach (2013) to compare the performance of our VaR models statistically.

We demonstrate that the results from the volatility forecast literature cannot be transposed to a quantile forecast application. Although IV information generally outperforms time series information for volatility prediction, our results suggest that this is not the case for VaR forecast. IV and IV adjusted VaR do not outperform a Glosten-Jagganathan and Runkle (1993) Garch based VaR (GJR-Garch VaR). The latter historical volatility model is even found, under certain circumstances, to outperform all the alternative models. The GJR-Garch passes the LR and dynamic quantile tests more successfully than the other VaR models including the IV based VaR. A model-to-model statistical loss function comparison shows that the GJR-Garch always belongs to the group of best models. We will demonstrate that the failure of IV to provide a better VaR than the historical volatility model is robust to changes in the VaR confidence level and the particular market under investigation. Moreover, the maturity mismatch between the VaR horizon and the option maturity cannot explain these results.

Because of the volatility risk premium, IV VaR severely overstates the actual VaR. This is translated into far too small a number of observed violations for the three indices. An inaccurate, excessively conservative $\mathrm{VaR}$ implies an excess capital charge for financial institutions. This finding draws attention to the need to account for the variance risk premium when options IV are used to forecast VaRs.

Relatively simple IV adjustments significantly correct and improve the volatility forecasting power of option implied information (Prokopczuk and Wese Simen, 2014; DeMiguel, Plyakha, Uppal and Vilkov, 2013). Although the parametric and non-parametric volatility risk premium adjustments we propose reduce the downward bias of the standard VaR IV, they do not allow IV to outperform the GJR-Garch model. While standard volatility risk premium adjustments are successful for volatility forecasting, they are not successful for quantile prediction. These adjustments assume a parsimonious linear relationship between the volatility risk premium or relative volatility risk premium and the volatility level. This parsimonious specification is enough to capture properly the future average volatility. However, a richer dynamic is necessary to predict the tail of returns distribution. Our results and other evidence found in the literature suggest that the relationships between the volatility risk premium, volatility, returns and innovations are highly non-linear around extreme events. Therefore, the linear assumptions are the inappropriate for predicting extreme quantiles of the return distribution. 


\section{CHAPTER 5}

We conclude that the use of option implied information for risk management purposes is not as efficient as was commonly supposed in the volatility forecasting literature. The volatility risk premium included in option implied information and the adjustment technique used do not provide sufficiently good VaR forecasts to beat simple historical volatility model based VaR forecasts.

\subsection{DATA}

We proxy the model free IVs of the S\&P 500, DJIA and NASDAQ 100 with their respective Chicago Board of Exchange (CBOE) volatility indices. They represent the investor riskneutral expectation of the next 30 days volatility for these three market indices. We rely on the new volatility indices that are computed from the whole range of options available rather than only the at-the-money options as was previously the case. This approach accounts for the implied volatility smile and skew featured by the option market. Corrado and Miller (2005) demonstrate empirically that the VXO, VIX and VXN are informationally efficient and that the infamous error-in-variable problem for these indices is no longer a real concern after 1995. These volatility indices are computed without relying on any model such as Black and Scholes (1973) and are therefore truly model-free measures. Martin et al. (2009) show that the VIX replicates very precisely the model-free measure of Britten-Jones and Neuberger (2000) and Jiang and Tian (2005).

We collect daily return series on the underlying equity indices from DataStream. The data cover different periods for the three indices, depending on the availability of the volatility indices which varies across indices. The sample covers the S\&P 500 from 1990, the DJIA from 1997 and NASDAQ from 2001 to 2013. Figure 5.1 shows some evident common trend across the three indices price levels and IVs. However, the three markets display different levels of volatility, NASDAQ 100 being the most volatile index. Table 5.1 compares the indices' volatility more formally. Both the 22 days realized volatilities and the IV are presented. Considering the time periods covered for the three markets (after 2002), the annualized realized volatility of the NASDAQ is respectively $4.7 \%$ and $5.9 \%$ higher than the S\&P and DJIA volatilities. During the earliest time period of our sample, the DJIA is more volatile than the S\&P but the situation reverses after 2002.

In the next section, we evaluate the benefits of IV, a risk neutral measure, for forming VaR forecasts for actual physical returns. Table 5.1 provides statistics regarding the spread between the risk neutral volatility and the physical volatility. As widely documented, the variance risk premium is negative and the IV surpasses the subsequent realized volatility (Carr and $\mathrm{Wu}, 2009$; Mixon, 2009). The average size of the premium is economically significant, amounting from $3.26 \%$ to $5.51 \%$. These premia are also statistically significant. All mean VRP reported in table 5.1, except for the NASDAQ before 2002, are significant at the $1 \%$ level using Newey-West standard errors. 
Forecasting Volatility or Value-at-Risk: when does the Volatility Risk Premium matter?

Table 5.1: Implied volatility, realized volatility and volatility risk premium

\begin{tabular}{|c|c|c|c|c|c|c|c|c|c|}
\hline & & Mean & AR1 & Median & STDev & Skew & Kurt & $5 \%$ & $95 \%$ \\
\hline \multirow[t]{12}{*}{$\overline{\mathrm{S \& P}}$} & \multicolumn{9}{|c|}{ Total Sample $(\mathrm{N}=5873)$} \\
\hline & IV & 20.36 & 0.98 & 18.72 & 8.12 & 2.00 & 10.10 & 11.52 & 34.82 \\
\hline & RV & 15.94 & 0.99 & 13.62 & 9.44 & 2.88 & 16.04 & 7.10 & 32.05 \\
\hline & VRP & 4.42 & 0.95 & 4.85 & 6.04 & -2.62 & 20.61 & -4.00 & 11.74 \\
\hline & \multicolumn{9}{|c|}{ Before $2002(\mathrm{~N}=3025)$} \\
\hline & IV & 19.46 & 0.98 & 18.75 & 6.11 & 0.83 & 3.69 & 11.61 & 30.45 \\
\hline & RV & 14.30 & 0.99 & 12.94 & 6.39 & 1.23 & 4.80 & 6.53 & 27.33 \\
\hline & VRP & 5.17 & 0.94 & 5.41 & 4.73 & -0.46 & 5.58 & -2.69 & 12.14 \\
\hline & \multicolumn{9}{|c|}{ After $2002(\mathrm{~N}=2848)$} \\
\hline & IV & 21.30 & 0.98 & 18.68 & 9.72 & 2.01 & 8.73 & 11.40 & 40.65 \\
\hline & RV & 17.68 & 1.00 & 14.29 & 11.60 & 2.67 & 12.39 & 7.76 & 39.71 \\
\hline & VRP & 3.63 & 0.95 & 4.26 & 7.09 & -3.00 & 19.83 & -6.26 & 11.48 \\
\hline \multirow{12}{*}{ DJIA } & \multicolumn{9}{|c|}{ Total Sample $(\mathrm{N}=3911)$} \\
\hline & $\overline{\mathrm{IV}}$ & 20.96 & 0.98 & 19.89 & 8.26 & 1.59 & 7.64 & 11.01 & 36.50 \\
\hline & RV & 17.09 & 0.99 & 14.77 & 9.71 & 2.55 & 12.78 & 7.51 & 35.98 \\
\hline & VRP & 3.87 & 0.96 & 4.49 & 6.60 & -2.12 & 13.94 & -6.35 & 11.74 \\
\hline & \multicolumn{9}{|c|}{ Before $2002(\mathrm{~N}=1063)$} \\
\hline & $\overline{\mathrm{IV}}$ & 24.21 & 0.96 & 23.46 & 4.55 & 1.08 & 4.73 & 18.40 & 33.07 \\
\hline & RV & 18.70 & 0.98 & 16.95 & 6.55 & 1.43 & 4.75 & 11.60 & 33.70 \\
\hline & VRP & 5.51 & 0.96 & 7.02 & 6.58 & -1.05 & 4.45 & -8.86 & 13.62 \\
\hline & \multicolumn{9}{|c|}{ After $2002(\mathrm{~N}=2848)$} \\
\hline & IV & 19.75 & 0.98 & 17.31 & 8.97 & 1.94 & 8.19 & 10.77 & 37.75 \\
\hline & RV & 16.49 & 1.00 & 13.34 & 10.59 & 2.68 & 12.64 & 7.27 & 37.01 \\
\hline & VRP & 3.26 & 0.95 & 3.81 & 6.51 & -2.65 & 17.77 & -5.55 & 10.77 \\
\hline \multirow[t]{12}{*}{ NSADAQ } & \multicolumn{9}{|c|}{ Total Sample (N=3072) } \\
\hline & IV & 28.15 & 0.99 & 23.67 & 13.03 & 1.25 & 3.79 & 15.07 & 56.35 \\
\hline & RV & 24.38 & 1.00 & 19.45 & 14.22 & 1.74 & 6.15 & 10.98 & 53.63 \\
\hline & VRP & 3.77 & 0.96 & 4.27 & 7.63 & -2.26 & 14.77 & -7.76 & 13.33 \\
\hline & \multicolumn{9}{|c|}{ Before $2002(\mathrm{~N}=226)$} \\
\hline & IV & 54.61 & 0.96 & 54.86 & 7.21 & 0.23 & 2.01 & 45.36 & 66.22 \\
\hline & RV & 49.43 & 0.99 & 46.08 & 13.96 & 0.90 & 2.93 & 32.12 & 79.78 \\
\hline & VRP & 5.18 & 0.96 & 6.96 & 11.20 & -0.55 & 2.64 & -16.71 & 20.91 \\
\hline & \multicolumn{9}{|c|}{ After $2002(\mathrm{~N}=2846)$} \\
\hline & IV & 26.04 & 0.99 & 22.82 & 10.91 & 1.49 & 5.18 & 14.97 & 48.69 \\
\hline & RV & 22.39 & 0.99 & 18.74 & 12.21 & 2.03 & 8.10 & 10.89 & 47.49 \\
\hline & VRP & 3.65 & 0.96 & 4.19 & 7.26 & -2.73 & 18.16 & -6.08 & 12.07 \\
\hline
\end{tabular}

otes: This table represents the Implied Volatilities from the VIX, VXD, VXN, the realized volatilities from the \&P 500, the DJIA, and the NASDAQ. The variables are collected or computed on a daily basis. The sampls overs 23,16 and 12 years of data respectively for the S\&P500, DJIA and NASDAQ. The realized volatilities ar omputed as the sum of the 22 subsequent squared log-returns. The Volatility Risk Premium is computed as (IV V). All figures are annualized and reported as percentages. 
Figure 5.1: Indices and implied volatilities levels
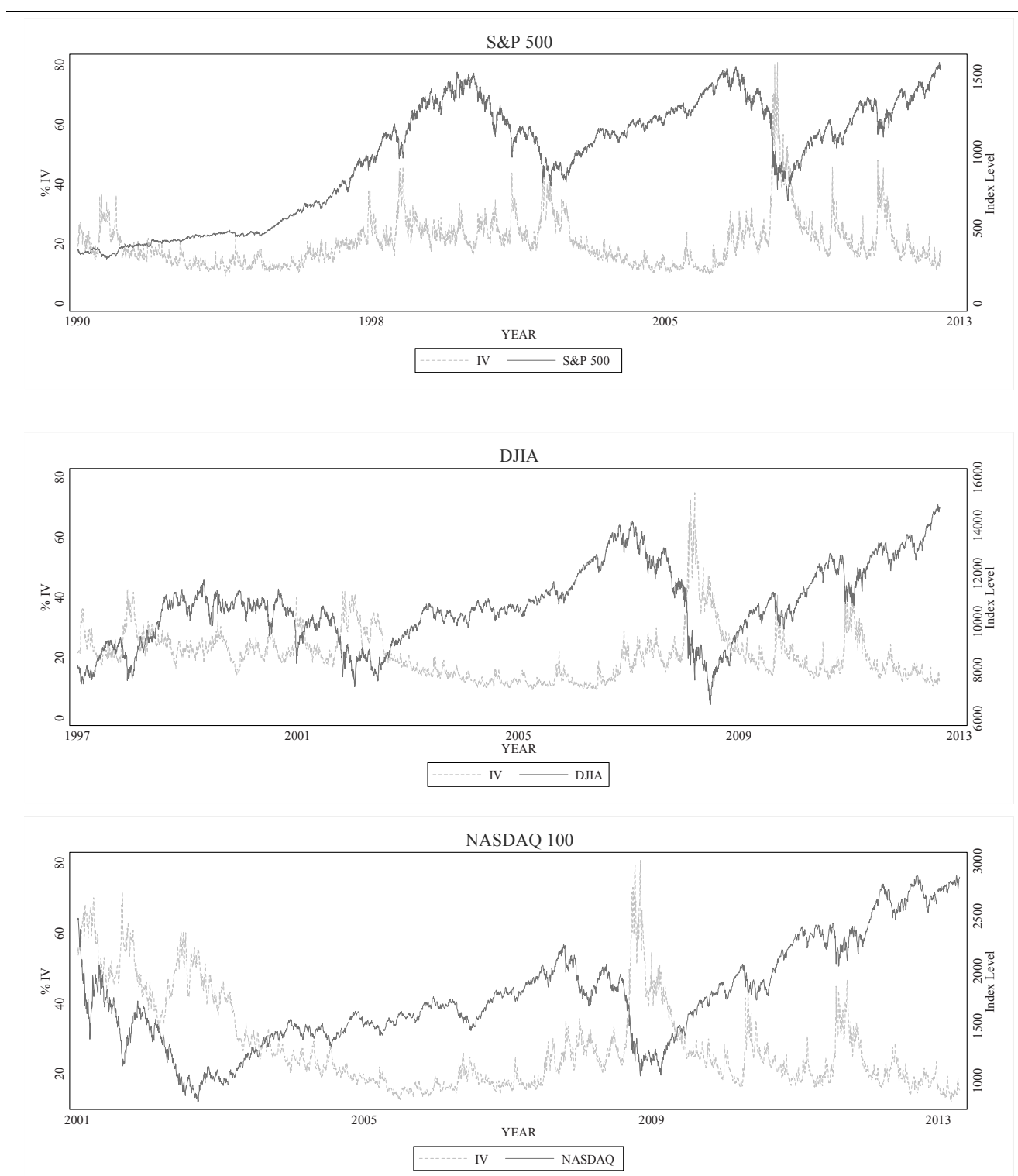

Notes: The figures show the time series of the index in dark solid grey and the implied volatility in light dashed grey. This is presented for the three indices under investigation: S\&P500, DJIA and NASDAQ. The time series does not have the same length because we are limited by the availability of the volatility indices. The time series only starts when the implied volatility is available. This explains why S\&P 500 has a longer time series than Nasdaq. The VIX is available from 1990 onwards while the VXN is only available after 2001. 
The ratio volatility risk premium over implied volatility is the lowest for the NASDAQ index. Also, the NASDAQ volatility risk premium is more volatile than the volatility risk premium of the other indices. On a smaller sample, the statistics of Corrado and Miller (2005) also show that the NASDAQ spread between IV and realized volatility is almost nonexistent and significantly lower than for other indices. They interpret the good predictive power of VXN on subsequent realized volatility as a sign of the NASDAQ 100 option market efficiency. But this can also be explained by the relatively smaller variance risk premium, making IV a less upwardly biased estimator for that specific market.

Aside from the economic importance of the price of volatility risk, table 5.1 shows that raw $\mathrm{IV}$ is an inappropriate input for a $\mathrm{VaR}$ forecast because of the volatility risk premium. An adjustment is therefore required, as suggested in DeMiguel et al. (2013) and Prokopczuk and Wise Simen (2014) or as suggested by Jorion (1995).

\subsection{METHODOLOGY}

The VaR at the $\alpha \%$ confidence level measures the loss of an investment that should not be exceeded in more than $\alpha \%$ of the cases. VaR forecasting boils down to predicting the $\alpha$ quantile of the return distribution. The 1-day $\mathrm{VaR}$ is used for our empirical application, and a one month horizon $\mathrm{VaR}$ is also tested for robustness purposes.

$\hat{\sigma}_{t}^{G J R}=\sqrt{\widehat{\omega}+\hat{\alpha} r_{t-1}^{2}+\hat{\gamma} r_{t-1}^{2} I_{\left[r_{t-1}<0\right]}+\hat{\beta} \sigma_{t-1}^{2}}$

\section{Implied Volatility Based Forecasts}

The first IV forecast simply uses the observed IV from the implied volatility indices at the end of day $t-1$. Because the volatility indices are computed for 30 days maturity options, that are commonly assumed to be 22 traded days, we adjust for the maturity as follows:

$\hat{\sigma}_{t}^{I V}=\sqrt{\frac{1}{22}} \times I V_{t-1}$

where the $I V_{t-1}$ are the VIX, VXD and VXD values at the end of day $t-1$.

This IV forecast does not acknowledge the discrepancy between volatility expectation under $\mathbb{P}$ and under $\mathbb{Q}$ but assumes that those two measures are equivalent. We correct for this difference by dividing $I V_{t}$ by an estimate of the ratio between implied volatility and realized volatility $\left(V R P_{t}\right)$. This ratio is estimated in two ways: parametrically and non-parametrically. The non-parametric adjustment is based on the historical median value of the ratio of the IV and the subsequent realized volatility computed as: 
$\widehat{V R P}_{t}(N P A)=I V_{t} / \sqrt{\sum_{i=t+1}^{t+23} r_{i}^{2}}$

Prokopczuk and Wise Simen (2014) propose using the ratio instead of the difference to account for the well known fact that variance risk premium is a function of the volatility level (Carr and $\mathrm{Wu}, 2009$; Martin et al., 2009). We compute the median of $V R P_{t}$ over the last 3 months. This window is a good compromise to capture time variation in the volatility risk premium and to estimate it sufficiently well. The non-parametrically adjusted IV forecast is denoted $\hat{\sigma}_{t}^{I V}(N P A)$.

This non-parametric adjustment is similar to the methodology provided by DeMiguel et al. (2013) and Prokopczuk and Wise Simen (2014). These two studies use the average relative ratio of IV over realized volatility over the last year. Instead, we rely on the median to shorten the period to three months and capture more time variation empirically observed in the variance risk premium (Bollerslev, Gibson and Zhou, 2011; Todorov, 2009). ${ }^{7}$

The alternative parametric adjustment estimates the implied volatility over realized volatility ratio from an $\operatorname{ARMA}(1,1)$ model:

$V R P_{t}=\varphi_{0}+\varphi_{1}$ RRPRatio $_{t-22}+\theta e_{t-22}+e_{t}$

where $\varphi_{1}$ represents the AR coefficient and the $\theta$ the MA coefficient. This model is estimated on the last 66 observations available ${ }^{8}$ ( 3 trading months) and provides an out-of-sample $\widehat{V R P}_{t}$ prediction. This forecast is used to construct the parametrically adjusted IV forecast $\hat{\sigma}_{t}^{I V}(N P A)$.

Table 5.2 summarises the 5 volatility forecasts that we use for estimating our VaR and provides some basic information about the major distinctions between those alternative methods. In the remainder of the chapter, we empirically evaluate and benchmark the performance of those approaches to provide accurate VaR measures.

So far, we have not provided any information on how we treat innovations, since we have only considered volatility forecasts. The performance of a VaR is highly affected by the choice of an appropriate distribution for the innovations (Mittnik and Paolella, 2000; Giot and

\footnotetext{
${ }^{7}$ We tried multiple horizons and time windows to compute the median VRP ratio, but the results are insensitive to this choice.

${ }^{8} 66$ observations to fit an ARMA model may appear a small number of observations even if the mean is a simpler moment to fit compared to the second moment. To alleviate this concern we varied the number of observations up to one year. The ARMA based VaR is slightly improved but not so much and the conclusions remain unchanged.
} 
Laurent, 2003). Including some form of non-normality and negative skewness in the innovation distribution is a necessary requirement for obtaining a reliable VaR.

Table 5.2: Summary volatility forecasts - time series versus implied volatility

\begin{tabular}{lcccc}
\hline Forecast & Name & $\begin{array}{c}\text { Time Serie / Implied } \\
\text { Volatility }\end{array}$ & Parametric & VRP bias \\
\hline Historical Realized Volatility & $\sigma_{t}^{R V}$ & Time Series & No & No \\
GJR Garch (1,1) & $\sigma_{t}^{G J R}$ & Time Series & Yes & No \\
Implied Volatility & $\sigma_{t}^{I V}$ & Implied Volatility & No & No \\
$\begin{array}{l}\text { Non Parametric Adjusted Implied } \\
\text { Volatility }\end{array}$ & $\sigma_{t}^{I V}(N P A)$ & Both & Corrected \\
Parametric Adjusted Implied Volatility & $\sigma_{t}^{I V}(P A)$ & Both & Yes & Corrected \\
\hline
\end{tabular}

Notes: The table summarizes the 5 different volatility forecasts presented in the methodology section. It highlights the property of every forecast with respect to the data it uses and whether it is a parametric forecast or not. Additional information is provided about the treatment of the VRP for implied volatility forecasts. We coined the column volatility risk premium (VRP) bias although this is not per se a bias but it represents a misalignment of the forecast with the VaR assessment.

To avoid making any arbitrary distributional choice we rely on the empirical innovation distribution. Barone-Adesi, Bourgoin and Giannopoulos (1998) proposed the filtered historical simulation technique to account for non-normality of innovations. In a similar spirit, we compute the conditional innovations dividing the realized returns by the forecast volatility of each model. The quantiles $(\alpha)$ of the previous 500 empirical innovations are used to form the current VaR. This approach, which drastically improves the performance of the VaR over a naive normality assumption, does not make any assumption about the innovation distribution $^{9}$ and is consistently applied across all models.

\subsection{RESULTS}

By the application of the methodology described in the previous section, we obtain five outof-sample VaR measures: $\operatorname{VaR}_{t}^{H V}, \operatorname{VaR}_{t}^{G J R}, \operatorname{VaR}_{t}^{I V}, \operatorname{VaR}_{t}^{I V}(N P A)$ and $\operatorname{VaR}_{t}^{I V}(P A)$. In this section, we analyse the properties and the performance of those measures. Figure 5.2 graphs the VaR levels and the returns for these different measures.

\footnotetext{
${ }^{9}$ The only implied assumption is that the innovation distribution is not time varying.
} 


\section{CHAPTER 5}

Figure 5.2: Returns and VaRs for the S\&P 500

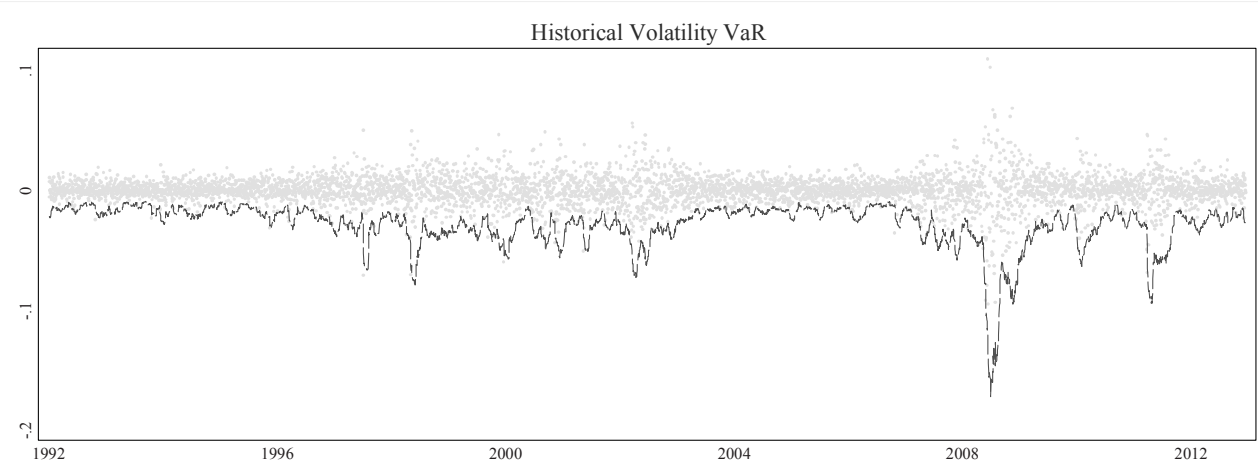

GJR Garch VaR

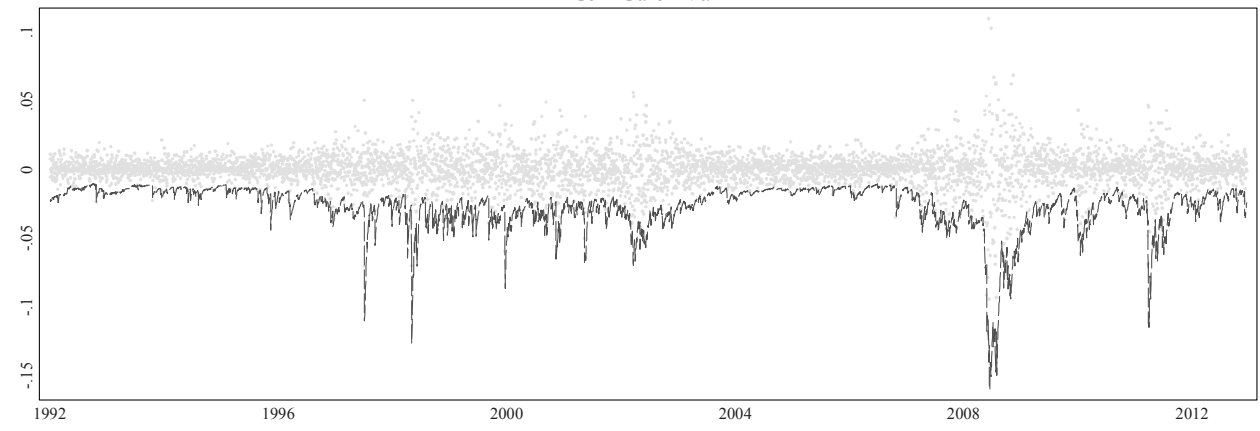

IV VaR

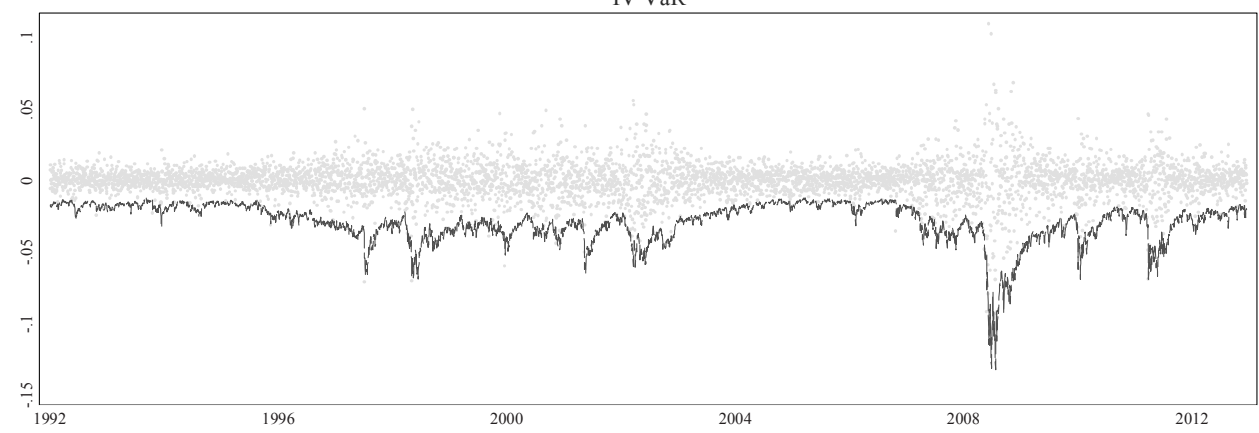



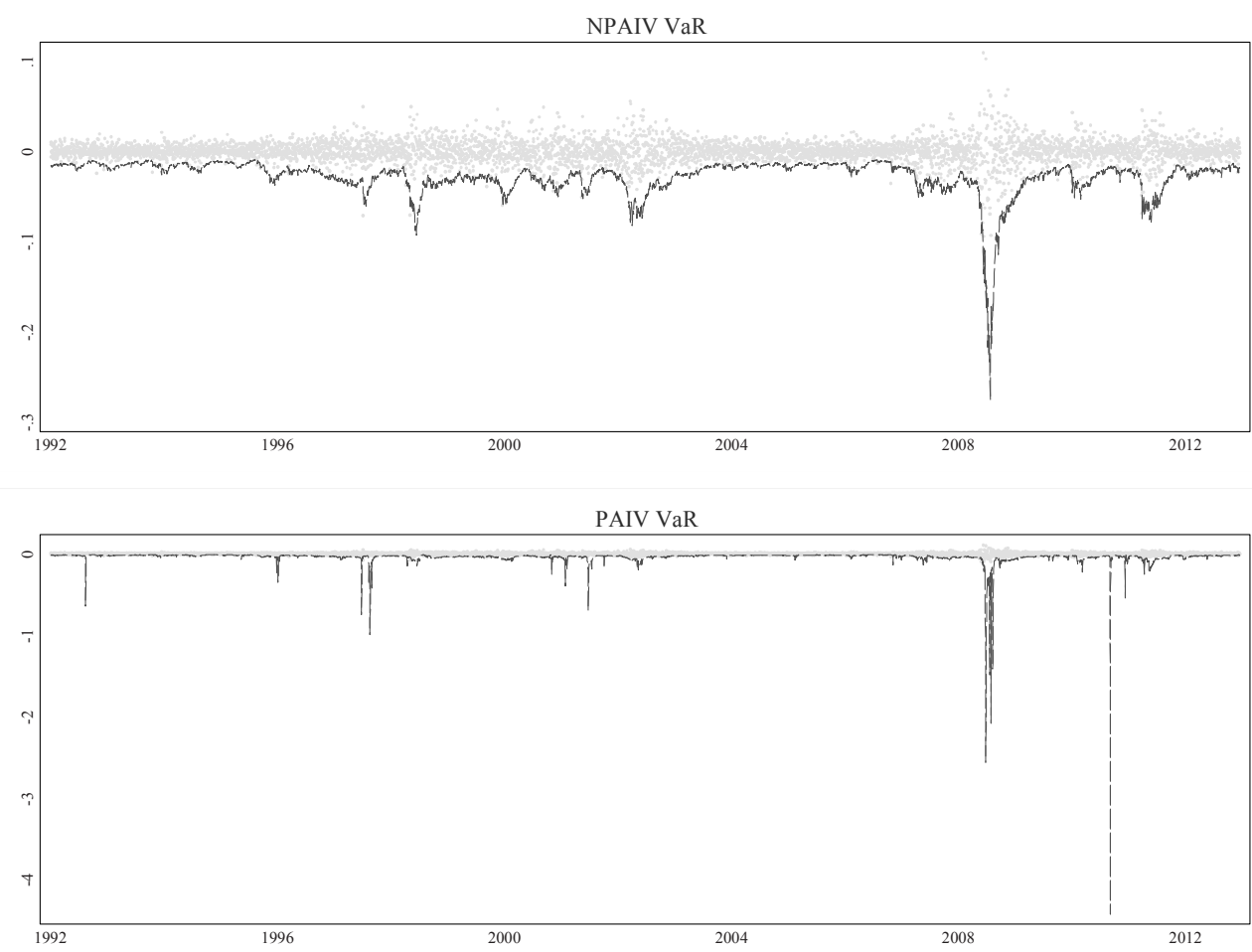

Notes:The figures show the time series of returns plotted as light grey dots and the time series of VaR, graphed as a dark grey continuous line, computed for different models. Each panel represent a different VaR. For the last panel, we have excluded one outlier that does not allow for a propergraphical observation because of its extreme value.

VaR measures deviate with respect to smoothness and confidence levels. Because of the extra weight applied to the most recent observations, $V a R^{G J R}$ is more responsive than the $V a R^{H V}$. The $V a R^{I V}$ also quickly includes new volatility information. The historical volatility model $\mathrm{VaR}$ and implied volatility $\mathrm{VaR}$ are distinct with respect to their level.VaR ${ }^{I V}$ is more negative than the alternative measures. The median $V a R_{t}^{H V}, V a R_{t}^{G J R}$ and $V a R_{t}^{I V}$ are respectively $-2.4 \%$, $-2.3 \%$ and $-2.7 \%$.

The IV adjustments provide lower absolute VaR values. The medians of $\operatorname{VaR}_{t}^{I V}(N P A)$ and $\operatorname{VaR}^{I V}(P A)$ are $-2.3 \%$ and $-2.5 \%$, respectively, indicating that the adjustments correct for the volatility risk premium efficiently. However, the extra times series information used for the non parametric adjustment causes the $\operatorname{VaR}^{I V}(N P A)$ to be excessively smoothed and to lag significantly. The parametric adjustment suffers from another type of shortcoming. The $\operatorname{ARMA}(1,1)$ volatility risk premium out-of-sample predictions assume unstable and occasionally extreme values. Thus, $\operatorname{VaR}^{I V}(N P A)$ forecasts are at times unrealistically high. Although the non-parametric approach, relying on a median, is arbitrary, it does not suffer from the uncertainty in the parameter estimates. 


\section{CHAPTER 5}

Next, we evaluate and back-test the competing VaR measures with particular attention to the relative performance of the time series historical volatility model approaches compared to the IV approaches. The VaR results are assessed with three complementary tests: the Kupiec test (1995), the dynamic quantile test (Engle and Manganelli, 2004) and the loss function test (Chen and Gerlach, 2009).

\section{Unconditional Test}

Table 5.3 reports the percentage violation (Vrate), the empirical percentage violation divided by the theoretical percentage violation (VrateRatio) and the Kupiec LR statistic for the two $\mathrm{VaR}$ levels. The violation rate ratio should be as close as possible to one. A bold LR statistic indicates that the VaR model fails the unconditional test.

Both the violation rate and the scaled violation rate show that $V a R^{I V}$ is too rarely violated for all markets and both VaR confidence levels. The LR test confirms statistically that the $V a R^{I V}$ is excessively conservative. The adjustments performed successfully provide a more accurate VaR level. Despite the volatility risk premium corrections the IV VaRs do not outperform the time series based VaRs. In fact, $V a R^{G J R}$ appears to be the best VaR model of all. In line with the results of Corrado and Miller (2005), the $\operatorname{VaR}^{I V}$ is the least biased for the NASDAQ market. They find that VXN is the volatility index that provides the least biased volatility forecast. This suggests that the higher the volatility risk premium in a given market, the more biased the IV based VaR for that market.

The non-parametric adjustment provides a better VaR than the parametric adjustment. The null hypothesis of the LR test for $\operatorname{VaR}^{I V}(N P A)$ is not rejected for two of the three markets. On the contrary, the null is rejected in all three markets for $\operatorname{VaR}^{I V}(P A)$. Because of the poor out-of-sample forecasting performance of the $\operatorname{ARMA}(1,1)$ model, the non-parametric approach is preferred.

The unconditional test shows that historical volatility VaRs models and IV adjusted VaRs provide very similar performance. In fact, $\mathrm{VaR}^{\mathrm{GJR}}$ appears to be the best VaR model of all. Further tests will help to support this claim.

\section{Dynamic Quantile Test}

The dynamic quantile test (DQ) proposed by Engel and Manganelli (2004) allows for assessing the conditional performance of VaR models. This test investigates whether the violation rate is independent from certain conditions such as past violations or volatility regime. For the DQ test the sequence of $\operatorname{VaR}_{t}^{x}$ is transformed into a Hit variable as follows: 


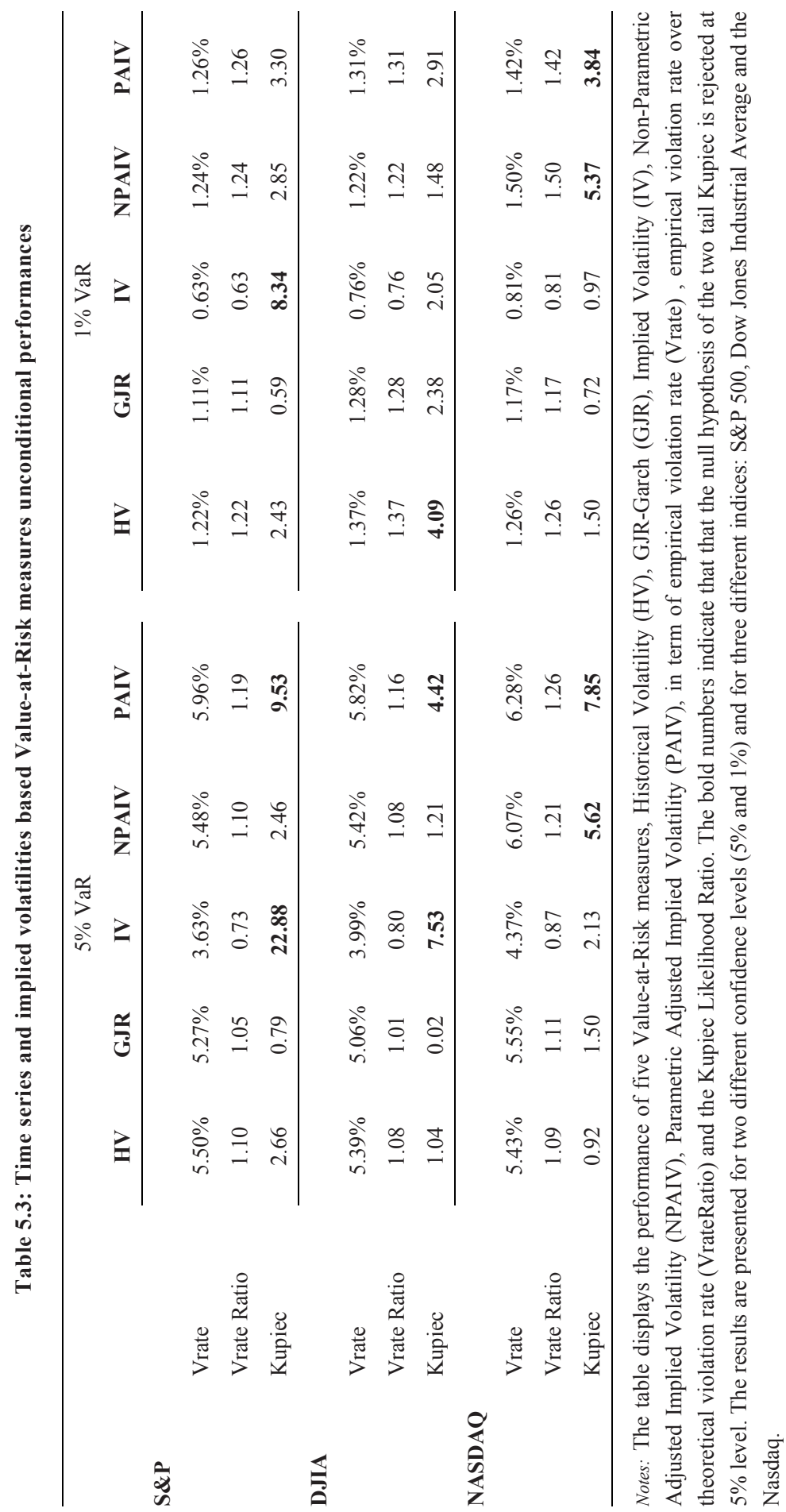




\section{CHAPTER 5}

$\operatorname{Hit}_{t}(x)=I\left[r_{t}<\operatorname{VaR}_{t}^{x}\right]-\alpha$

where the indicator variable $I$ takes the value of one when $\operatorname{VaR} R_{t}^{x}$ is violated. This implies that $H_{i t}(x)$ takes the value of 1 minus the VaR confidence level when a violation occurs and minus the VaR confidence level when there is no violation. For a correctly specified VaR, the expectation of $\operatorname{Hit}_{t}(x)$ is zero. In a linear regression framework, $\operatorname{Hit}_{t}(x)$ is regressed on its lags, to test independence, and any other relevant lagged variables. The probability of a VaR model being violated should remain unchanged under any condition. Hence all coefficients in the regression should be equal to zero. Engel and Manganelli (2004) propose for this purpose the conditional coverage test statistic:

$D Q_{\text {oos }}=\frac{\hat{B}^{\prime} X^{\prime} X \hat{\hat{B}}}{\alpha(1-\alpha)}$

The statistic is $\chi^{2}(K)$ distributed. $\hat{B}$ is the $K \mathrm{x} 1$ column vector of the estimated coefficients and $X$ is a $N \times K$ matrix where $N$ represents the number of observations in the regression.

The lagged Hit variable tests for independence. The lagged VaR and squared return verify whether the violation rate is constant across different volatility regimes. The lagged change in IV identifies whether violation is affected by a sudden change in volatility.

Table 5.4 reports the results of the dynamic quantile tests. The $V_{a}{ }^{G J R}$ passes successfully the conditional efficiency tests five out of six times across markets and VaR confidence levels. The other VaRs, including the $\mathrm{VaR}^{\mathrm{IV}}$ and $\mathrm{VaR}^{\mathrm{IV}}$ (NPA), almost always fail the test. The violation rate of the $\mathrm{VaR}^{\mathrm{GJR}}$ is the least affected by the market conditions.

The significance of the parameter estimates is indicative of the relative strength and weakness of each VaR model. The effect of the variance risk premium also appears in the regression results. The parameter estimates of the constants indicate a tendency of $\mathrm{VaR}^{\mathrm{IV}}$ to overestimate risk while other VaRs tend to underestimate risk. The lagged dependent variable coefficients are always significant, indicating that violations are clustered for every VaR model. Although forward looking and theoretically more reactive to new information, the IV based VaRs are not performing better than their competitors regarding this criterion. The lagged VaR and lagged squared return are more often significant and more significant for $\mathrm{VaR}^{\mathrm{HV}}$, $\mathrm{VaR}^{\mathrm{IV}}$ (NPA) or $\mathrm{VaR}^{\mathrm{IV}}(\mathrm{PA})$. This indicates that the likelihood of a violation being observed is higher in high volatility regimes. This undesirable effect is attenuated for $V a R^{G J R}$. 
Forecasting Volatility or Value-at-Risk: when does the Volatility Risk Premium matter?

Table 5.4: Dynamic quantile tests

\begin{tabular}{|c|c|c|c|c|c|c|c|c|c|c|}
\hline \multirow{2}{*}{ S\&P } & \multicolumn{5}{|c|}{$5 \% \mathrm{VaR}$} & \multicolumn{5}{|c|}{$1 \% \mathrm{VaR}$} \\
\hline & $\underline{\text { HV }}$ & GJR & IV & \multicolumn{2}{|c|}{ NPAIV PAIV } & $\underline{\text { HV }}$ & GJR & IV & \multicolumn{2}{|c|}{ NPAIV PAIV } \\
\hline \multirow[t]{2}{*}{ Constant } & 0.02 & 0.01 & -0.01 & 0.02 & 0.01 & 0.01 & 0.01 & -0.01 & 0.01 & 0.00 \\
\hline & 3.03 & 2.12 & -1.82 & 3.24 & 2.92 & 3.28 & 1.95 & -2.47 & 2.61 & 1.75 \\
\hline \multirow[t]{2}{*}{ Lag Hit } & 0.01 & 0.00 & -0.01 & -0.01 & 0.00 & 0.00 & 0.01 & 0.00 & 0.02 & 0.00 \\
\hline & 0.70 & -0.24 & -0.41 & -0.88 & -0.14 & -0.26 & 0.36 & 0.22 & 0.83 & 0.21 \\
\hline \multirow[t]{2}{*}{ Lag 2 Hit } & 0.06 & 0.03 & 0.06 & 0.05 & 0.08 & 0.02 & 0.03 & 0.05 & 0.02 & 0.08 \\
\hline & 3.02 & 1.73 & 2.78 & 2.68 & 3.99 & 0.93 & 1.11 & 1.52 & 0.91 & 2.21 \\
\hline \multirow[t]{2}{*}{ Lag 3 Hit } & 0.00 & -0.01 & 0.00 & 0.01 & 0.05 & -0.01 & -0.01 & -0.01 & -0.01 & 0.02 \\
\hline & 0.25 & -0.85 & -0.19 & 0.87 & 2.70 & -7.49 & -5.99 & -3.16 & -6.20 & 0.86 \\
\hline \multirow[t]{2}{*}{ Lag 4 Hit } & 0.01 & 0.00 & 0.03 & 0.02 & 0.04 & -0.01 & 0.01 & 0.02 & 0.02 & 0.04 \\
\hline & 0.83 & 0.24 & 1.56 & 1.52 & 1.98 & -7.58 & 0.46 & 0.82 & 0.89 & 1.68 \\
\hline \multirow[t]{2}{*}{ Lag VaR } & 0.81 & 0.43 & -0.02 & 0.62 & 0.00 & 0.28 & 0.17 & -0.19 & 0.16 & 0.00 \\
\hline & 2.36 & 1.54 & -0.05 & 2.81 & 3.98 & 3.16 & 1.65 & -1.39 & 2.36 & 2.72 \\
\hline \multirow[t]{2}{*}{ Lag Squared return } & -0.41 & -0.48 & -0.30 & -0.41 & -0.23 & -0.22 & -0.11 & -0.02 & -0.09 & -0.03 \\
\hline & -1.73 & -2.05 & -1.36 & -1.74 & -0.92 & -2.14 & -1.31 & -0.21 & -0.90 & -0.26 \\
\hline \multirow[t]{2}{*}{ Lag Change Vix } & -8.94 & -14.17 & -8.14 & -8.32 & -15.82 & 1.22 & -1.40 & -4.95 & -1.66 & -4.47 \\
\hline & -2.13 & -2.79 & -2.47 & -2.43 & -4.10 & 0.59 & -0.57 & -1.96 & -0.79 & -2.74 \\
\hline Adj_R2 & 0.01 & 0.00 & 0.01 & 0.00 & 0.01 & 0.00 & 0.00 & 0.01 & 0.00 & 0.01 \\
\hline DQoos P-Value & 0.00 & 0.01 & 0.00 & 0.00 & 0.00 & 0.00 & 0.21 & 0.00 & 0.02 & 0.00 \\
\hline
\end{tabular}

DJIA

\begin{tabular}{|c|c|c|c|c|c|c|c|c|c|c|}
\hline \multirow[t]{2}{*}{ Constant } & 0.02 & 0.01 & -0.01 & 0.01 & 0.01 & 0.01 & 0.01 & 0.00 & 0.01 & 0.00 \\
\hline & 2.53 & 0.83 & -1.33 & 2.28 & 2.07 & 3.07 & 2.50 & 0.10 & 2.18 & 1.60 \\
\hline \multirow[t]{2}{*}{ Lag Hit } & 0.02 & 0.02 & 0.03 & 0.02 & 0.04 & 0.05 & -0.01 & -0.01 & 0.01 & -0.01 \\
\hline & 1.13 & 0.89 & 1.22 & 0.90 & 1.99 & 1.36 & -2.87 & -1.49 & 0.56 & -2.61 \\
\hline \multirow[t]{2}{*}{ Lag 2 Hit } & 0.05 & 0.01 & 0.04 & 0.05 & 0.05 & 0.03 & -0.01 & 0.07 & 0.04 & 0.06 \\
\hline & 2.05 & 0.54 & 1.67 & 2.33 & 2.04 & 0.94 & -5.49 & 1.06 & 1.18 & 1.66 \\
\hline \multirow[t]{2}{*}{ Lag 3 Hit } & 0.01 & -0.03 & 0.01 & 0.01 & 0.05 & -0.02 & -0.01 & -0.01 & -0.01 & 0.01 \\
\hline & 0.42 & -2.99 & 0.31 & 0.40 & 2.46 & -4.20 & -5.82 & -4.04 & -4.72 & 0.54 \\
\hline \multirow[t]{2}{*}{ Lag 4 Hit } & 0.01 & 0.02 & 0.04 & 0.02 & 0.08 & -0.01 & 0.01 & 0.03 & -0.01 & 0.01 \\
\hline & 0.60 & 0.90 & 1.92 & 1.15 & 2.54 & -4.38 & 0.54 & 1.02 & -3.89 & 0.31 \\
\hline \multirow[t]{2}{*}{ Lag VaR } & 0.85 & 0.23 & -0.17 & 0.51 & 0.02 & 0.32 & 0.26 & 0.10 & 0.18 & 0.00 \\
\hline & 2.14 & 0.56 & -0.45 & 1.86 & 1.77 & 2.96 & 2.27 & 0.99 & 2.34 & 1.57 \\
\hline \multirow[t]{2}{*}{ Lag Squared return } & 0.02 & 0.16 & 0.32 & 0.13 & 0.58 & -0.12 & -0.04 & -0.02 & 0.03 & 0.05 \\
\hline & 0.09 & 0.61 & 1.15 & 0.45 & 2.07 & -1.07 & -0.30 & -0.13 & 0.27 & 0.43 \\
\hline \multirow[t]{2}{*}{ Lag Change IV } & -8.18 & -12.76 & -12.81 & -10.13 & -16.23 & -0.39 & 2.50 & 2.10 & 0.42 & -3.88 \\
\hline & -1.60 & -2.30 & -2.60 & -1.98 & -3.21 & -0.15 & 0.91 & 0.80 & 0.19 & -2.14 \\
\hline Adj_R2 & 0.00 & 0.00 & 0.01 & 0.00 & 0.01 & 0.01 & 0.00 & 0.00 & 0.00 & 0.00 \\
\hline DQoos P-Value & 0.00 & 0.31 & 0.01 & 0.00 & 0.00 & 0.00 & 0.26 & 0.02 & 0.08 & 0.01 \\
\hline \multicolumn{11}{|l|}{ ASDAQ } \\
\hline \multirow[t]{2}{*}{ Constant } & 0.03 & 0.02 & 0.00 & 0.03 & 0.01 & 0.01 & 0.01 & 0.00 & 0.01 & 0.00 \\
\hline & 2.86 & 1.89 & -0.21 & 3.32 & 2.39 & 2.52 & 1.97 & 0.02 & 2.50 & 1.54 \\
\hline \multirow[t]{2}{*}{ Lag Hit } & -0.04 & -0.04 & -0.01 & -0.03 & 0.00 & -0.01 & -0.01 & 0.00 & -0.01 & 0.00 \\
\hline & -1.70 & -2.05 & -0.66 & -1.41 & 0.00 & -2.95 & -2.90 & -0.18 & -1.69 & -0.10 \\
\hline \multirow[t]{2}{*}{ Lag 2 Hit } & 0.06 & 0.03 & 0.07 & 0.06 & 0.09 & 0.02 & -0.01 & 0.04 & 0.06 & 0.07 \\
\hline & 2.26 & 1.48 & 2.11 & 2.44 & 3.34 & 0.62 & -4.82 & 0.93 & 1.26 & 1.33 \\
\hline Lag 3 Hit & 0.02 & 0.02 & 0.01 & 0.02 & 0.05 & -0.01 & -0.01 & -0.01 & 0.02 & 0.13 \\
\hline
\end{tabular}




\begin{tabular}{|c|c|c|c|c|c|c|c|c|c|c|}
\hline & \multicolumn{5}{|c|}{$5 \% \mathrm{VaR}$} & \multicolumn{5}{|c|}{$1 \% \mathrm{VaR}$} \\
\hline & \multirow{2}{*}{$\frac{\mathbf{H V}}{0.96}$} & \multirow{2}{*}{\begin{tabular}{|l|} 
GJR \\
0.75
\end{tabular}} & \multirow{2}{*}{$\frac{\mathbf{I V}}{0.36}$} & \multicolumn{2}{|c|}{ NPAIV PAIV } & \multirow{2}{*}{$\frac{\mathbf{H V}}{-4.92}$} & \multirow{2}{*}{$\begin{array}{c}\text { GJR } \\
-4.99\end{array}$} & \multirow{2}{*}{$\begin{array}{l}\text { IV } \\
-3.45\end{array}$} & \multicolumn{2}{|c|}{ NPAIVPAIV } \\
\hline & & & & 0.85 & 2.08 & & & & 0.61 & 2.01 \\
\hline \multirow[t]{2}{*}{ Lag 4 Hit } & 0.02 & 0.03 & 0.03 & 0.01 & 0.02 & -0.01 & -0.01 & -0.01 & 0.09 & 0.04 \\
\hline & 0.83 & 1.26 & 1.01 & 0.42 & 0.64 & -5.40 & -4.84 & -1.99 & 2.17 & 1.08 \\
\hline \multirow[t]{2}{*}{ Lag VaR } & 1.04 & 0.55 & 0.13 & 1.03 & 0.21 & 0.26 & 0.24 & 0.05 & 0.21 & 0.03 \\
\hline & 2.85 & 1.48 & 0.33 & 2.81 & 2.88 & 2.45 & 1.85 & 0.36 & 1.95 & 2.06 \\
\hline \multirow[t]{2}{*}{ Lag Squared return } & -0.33 & -0.39 & 0.23 & -0.15 & 0.50 & -0.03 & -0.11 & 0.09 & 0.09 & 0.01 \\
\hline & -1.29 & -1.41 & 0.79 & -0.41 & 1.58 & -0.23 & -1.02 & 0.93 & 0.73 & 0.05 \\
\hline \multirow[t]{2}{*}{ Lag Change IV } & 1.68 & -2.35 & -3.30 & 6.82 & 12.40 & 1.05 & 0.52 & -1.80 & -1.88 & 1.85 \\
\hline & 0.40 & -0.43 & -0.88 & 1.52 & 1.28 & 0.62 & 0.29 & -0.93 & -0.94 & 0.59 \\
\hline Adj_R2 & $\overline{0.01}$ & 0.00 & 0.00 & 0.01 & 0.02 & 0.00 & 0.00 & 0.00 & 0.01 & 0.02 \\
\hline DQoos P-Value & 0.00 & 0.08 & 0.03 & 0.00 & 0.00 & 0.24 & 0.57 & 0.66 & 0.00 & 0.00 \\
\hline
\end{tabular}

\section{Loss Function}

The two previous tests assess the performance of each VaR model individually. This evaluation highlights certain differences across models but does not allow formal evaluation of the relative performance of one model against another. In order to compare directly the performance of an IV based versus a historical based volatility model VaR, we adopt a loss function evaluation approach. Chen and Gerlach (2013) propose a loss function for VaR models inspired by the quantile regression loss function from Koenker and Bassett (1978). The loss function is:

$L F=\sum_{t=1}^{N}\left(r_{t}-\operatorname{VaR}_{t}\right)\left(\alpha-I_{t}\right)$

where $r_{t}$ is the return at time t, $V a R_{t}$ the forecast of a given VaR model for time $\mathrm{t}$ and $I_{t}$ an indicator function taking the value of 1 if the $V a R_{t}$ is violated at time t, i.e. $r_{t}<V a R_{t}$, and 0 otherwise. Making any inferences based on an absolute loss function comparison is not appropriate. Chen and Gerlach (2013) propose block bootstrapping the loss function in order to obtain the distribution of this statistic and test whether the loss function of one VaR model is statistically different from another.

We block bootstrap the difference between two VaR models loss functions to test for the loss function difference significance. A number of 1,000 replications with a block length of $N^{\left(\frac{1}{3}\right)}$ is used. $^{10}$ Table 5.5 reports the loss function for every VaR model, VaR level and index. The loss function pair wise difference across models is presented. The statistically significant differences, according to the bootstrapped distribution with a $95 \%$ confidence interval, are indicated in bold. A negative figure means that the loss function of the model on the horizontal line is lower, and therefore preferred, over the model on the vertical line.

\footnotetext{
${ }^{10}$ The block length is different from one index to another in order to take into account the differences in size of the sample.
} 
Forecasting Volatility or Value-at-Risk: when does the Volatility Risk Premium matter?

Table 5.5: Loss function and loss function difference tests

\begin{tabular}{|c|c|c|c|c|c|c|c|c|c|}
\hline \multirow{2}{*}{$\mathbf{S \& P}$} & \multicolumn{4}{|c|}{$5 \%$ VaR } & \multicolumn{5}{|c|}{$1 \%$ VaR } \\
\hline & $\underline{\mathrm{HV}}$ & GJR & IV & NPAIV PAIV & $\underline{\mathrm{HV}}$ & GJR & IV & NPAIV & PAIV \\
\hline $\begin{array}{l}\text { Loss } \\
\text { Function }\end{array}$ & 6.45 & 6.28 & 6.26 & 13.93 & 1.91 & 1.77 & 1.79 & 1.86 & 4.60 \\
\hline
\end{tabular}

Differences

\begin{tabular}{|c|c|c|c|c|c|c|c|c|c|c|c|}
\hline & HV & GJR & IV & \multicolumn{2}{|c|}{ NPAIV PAIV } & \multirow[b]{2}{*}{ HV } & \multirow{2}{*}{$\frac{\mathbf{H V}}{0.00}$} & \multirow{2}{*}{$\begin{array}{l}\text { GJR } \\
\mathbf{0 . 1 4}\end{array}$} & \multirow{2}{*}{$\begin{array}{l}\text { IV } \\
0.12\end{array}$} & \multicolumn{2}{|c|}{ NPAIV PAIV } \\
\hline HV & 0.00 & 0.18 & 0.20 & 0.09 & -7.45 & & & & & 0.06 & -2.67 \\
\hline GJR & -0.18 & 0.00 & 0.02 & -0.09 & -7.62 & GJR & -0.14 & 0.00 & -0.02 & -0.09 & -2.81 \\
\hline IV & -0.20 & -0.02 & 0.00 & -0.11 & -7.64 & IV & -0.12 & 0.02 & 0.00 & -0.06 & -2.79 \\
\hline NPAIV & -0.09 & 0.09 & 0.11 & 0.00 & -7.54 & NPAIV & -0.06 & 0.09 & 0.06 & 0.00 & -2.73 \\
\hline PAIV & 7.45 & 7.62 & 7.64 & 7.54 & 0.00 & PAIV & 2.67 & 2.81 & 2.79 & 2.73 & 0.00 \\
\hline
\end{tabular}

DJIA

\begin{tabular}{|c|c|c|c|c|c|c|c|c|c|}
\hline & $\underline{\mathrm{HV}}$ & GJR & IV & NPAIV PAIV & $\underline{\mathrm{HV}}$ & GJR & IV & NPAIV & PAIV \\
\hline $\begin{array}{l}\text { Loss } \\
\text { Function }\end{array}$ & 4.21 & 4.06 & 4.06 & 4.14 & 1.25 & 1.16 & 1.17 & 1.19 & 1.78 \\
\hline
\end{tabular}

Loss Function

Differences

\begin{tabular}{|c|c|c|c|c|c|c|c|c|c|c|c|}
\hline & HV & GJR & IV & \multicolumn{2}{|c|}{ NPAIV PAIV } & \multirow[b]{2}{*}{ HV } & \multirow{2}{*}{$\frac{\text { HV }}{0.00}$} & \multirow{2}{*}{$\begin{array}{l}\text { GJR } \\
\mathbf{0 . 0 9}\end{array}$} & \multirow{2}{*}{$\begin{array}{l}\text { IV } \\
0.08\end{array}$} & \multicolumn{2}{|c|}{ NPAIV PAIV } \\
\hline HV & $\overline{0.00}$ & 0.16 & 0.16 & 0.08 & -1.44 & & & & & 0.06 & -0.53 \\
\hline GJR & -0.16 & 0.00 & 0.00 & -0.08 & -1.60 & GJR & -0.09 & 0.00 & 0.00 & -0.02 & -0.62 \\
\hline IV & -0.16 & 0.00 & 0.00 & -0.08 & -1.60 & IV & -0.08 & 0.00 & 0.00 & -0.02 & -0.61 \\
\hline NPAIV & -0.08 & 0.08 & 0.08 & 0.00 & -1.52 & NPAIV & -0.06 & 0.02 & 0.02 & 0.00 & -0.59 \\
\hline PAIV & 1.44 & 1.60 & 1.60 & 1.52 & 0.00 & PAIV & 0.53 & 0.62 & 0.61 & 0.59 & 0.00 \\
\hline & \multicolumn{5}{|c|}{$\begin{array}{l}\text { NASDAQ } \\
\end{array}$} & & $\underline{\text { HV }}$ & GJR & IV & NPAIV & PAIV \\
\hline $\begin{array}{l}\text { Loss } \\
\text { Function }\end{array}$ & 3.69 & 3.61 & 3.58 & 3.64 & 4.60 & & 1.05 & 0.98 & 0.98 & 1.01 & 1.44 \\
\hline
\end{tabular}

Loss Function

Differences

\begin{tabular}{|c|c|c|c|c|c|c|c|c|c|c|c|}
\hline & HV & GJR & IV & NPAI & PAIV & & $\underline{\text { HV }}$ & GJR & IV & NPAIV & PAIV \\
\hline HV & 0.00 & 0.08 & 0.11 & 0.05 & -0.92 & HV & 0.00 & 0.07 & 0.07 & 0.04 & -0.39 \\
\hline GJR & -0.08 & 0.00 & 0.02 & -0.03 & -1.00 & GJR & -0.07 & 0.00 & 0.00 & -0.03 & -0.46 \\
\hline IV & -0.11 & -0.02 & 0.00 & -0.06 & -1.02 & IV & -0.07 & 0.00 & 0.00 & -0.03 & -0.46 \\
\hline NPAIV & -0.05 & 0.03 & 0.06 & 0.00 & -0.97 & NPAIV & -0.04 & 0.03 & 0.03 & 0.00 & -0.43 \\
\hline PAIV & 0.92 & 1.00 & 1.02 & 0.97 & 0.00 & PAIV & 0.39 & 0.46 & 0.46 & 0.43 & 0.00 \\
\hline
\end{tabular}

Notes: The table displays the value of the loss functions for the five VaR measures over three indices. The smaller the loss function, the better the VaR measure. In order to compare the loss functions across models, we compute the difference between the loss functions provided by a model on the vertical column and the loss function of the horizontal column. A block bootstrap is performed in order to test for that statistical significance of the loss function difference. The block length is chosen according to the following rule of thumb (numberofobservations) $^{1 / 3}$. We rely on 1,000 bootstraps.

Despite the change in the evaluation criteria, the same conclusions are reached. $V a R^{G J R}$ dominantly minimizes the loss function. In a statistical sense, historical volatility models are not outperformed by IV information for the purpose of VaR forecasting. $\mathrm{VaR}^{\mathrm{GJR}}, \mathrm{VaR}^{\mathrm{IV}}$ and $\mathrm{VaR}^{\mathrm{IV}}$ (NPA) do not have statistically different loss functions. The statistical test identifies $\mathrm{VaR}^{\mathrm{HV}}$ and $\mathrm{VaR}^{\mathrm{IV}}(\mathrm{PA})$ as the worst alternatives since they are always statistically outperformed. The loss function accounts for both the number of violations and the magnitude of the difference between the $\mathrm{VaR}$ and realized returns. The second factor favours the $V a R^{I V}$ 


\section{CHAPTER 5}

and heavily penalizes the $\operatorname{VaR}^{I V}(N P A)$. Although $\operatorname{VaR}^{I V}$ is too rarely violated, the differences between $r_{t}$ and $V_{a} R_{t}^{I V}$ are small. In contrast, the extreme differences between $r_{t}$ and $\operatorname{VaR}_{t}^{I V}(N P A)$, caused by the unstable $\operatorname{VaR}_{t}^{I V}(N P A)$ forecasts, explain the $\operatorname{VaR}^{I V}(N P A)$ underperformance.

\section{Robustness: Maturity mismatch}

The results presented contradict the forecasting volatility literature. Maturity mismatch is a factor that has been widely cited as impairing the forecasting ability of IV (Christensen and Prabhala, 1998; Yu et al., 2010). We evaluate the models on their ability to provide an accurate 1 day out-of-sample VaR; however, volatility indices represent the market expectation volatility over the next 30 days and not over the next day. Although the volatility term structure implied from options has not been found very steep (Stein, 1989; Christoffersen, Heston and Jacobs, 2013), a completely flat term structure cannot be assumed.

Unfortunately, the volatility indices are only available for this maturity. ${ }^{11}$ It is also not possible to compute accurately a model free implied volatility from very short maturity options because of lack of liquidity for these contracts. The relative success of the $\mathrm{VaR}^{\mathrm{GJR}}$ can be explained by its ability to adapt to different horizons. In order to verify this hypothesis, we replicate the previous analysis for 1 month out-of-sample VaRs. The GJR-Garch tends to revert quickly to the long run volatility and overly smooth long term forecast. Furthermore, the volatility forecasting literature highlights the high sensitivity of the results to the choice of the forecasting horizon (Ederington and Guan, 2002; Chen and Fung, 2012; Frijns et al., 2012). Although 1 month VaR is neither standard nor required by the regulator, it is a proper test for the robustness of our results.

The monthly unconditional tests, presented in Table 5.6, are aligned to the 1 day results of Table 5.3. We observe a convergence amongst the performance of $\mathrm{VaR}^{\mathrm{HV}}, \mathrm{VaR}^{\mathrm{IV}}, \mathrm{VaR}^{\mathrm{GJR}}$ and $\mathrm{VaR}^{\mathrm{IV}}$ (NPA). Repeatedly there is no clear evidence that IV based VaRs outperform historical volatility model VaRs. VaR ${ }^{\mathrm{GJR}}$ is no longer dominating the debate. But IV based VaRs still do not provide a better alternative.

As shown in Table 5.7, most VaR models are failing the conditional efficiency test for the $1 \%$ level VaR. The non-overlapping lag hit variables are strongly significant. The likelihood of other VaR models being violated is fairly unaffected by the remaining variables.

Table 5.8 presents the monthly VaR models loss functions. The good performance of VaR ${ }^{\mathrm{GJR}}$ in terms of loss function is robust to the change of maturity. $\mathrm{VaR}^{\mathrm{GJR}}$ minimizes the loss

\footnotetext{
${ }^{11}$ Recently the CBOE launched a new volatility index for a shorter maturity. The VXST, which is the VIX equivalent for 9 days maturity options, was launched only in 2011.
} 
function in 23 of the 24 pair wise comparisons. This superiority is 16 times statistically significant. It can be concluded that the relatively good performance of time series information compared to IV information in the VaR context is not explained by a maturity mismatch.

Table 5.6: Time series and implied volatilities based monthly Value-at-Risk measures unconditional performances

\begin{tabular}{|c|c|c|c|c|c|c|c|c|c|c|c|}
\hline \multirow{3}{*}{ S\&P } & & \multicolumn{5}{|c|}{$5 \% \mathrm{VaR}$} & \multicolumn{5}{|c|}{$1 \% \mathrm{VaR}$} \\
\hline & & HV & GJR & IV & NPAIV & PAIV & HV & GJR & IV & NPAIV & PAIV \\
\hline & & & & & & & & & & & \\
\hline & Vrate & $4.77 \%$ & $4.20 \%$ & $2.83 \%$ & $4.87 \%$ & $4.89 \%$ & $0.99 \%$ & $0.92 \%$ & $0.55 \%$ & $1.07 \%$ & $1.11 \%$ \\
\hline & $\begin{array}{l}\text { Vrate } \\
\text { Ratio }\end{array}$ & 0.95 & 0.84 & 0.57 & 0.97 & 0.98 & 0.99 & 0.92 & 0.55 & 1.07 & 1.11 \\
\hline & Kupiec & 1 & 7 & 61 & 0 & 0 & 0 & 0 & 13 & 0 & 1 \\
\hline \multicolumn{12}{|c|}{ DJIA } \\
\hline & Vrate & $5.33 \%$ & $4.66 \%$ & $3.63 \%$ & $5.55 \%$ & $5.73 \%$ & $1.58 \%$ & $1.34 \%$ & $1.07 \%$ & $1.37 \%$ & $1.40 \%$ \\
\hline & $\begin{array}{l}\text { Vrate } \\
\text { Ratio }\end{array}$ & 1.07 & 0.93 & 0.73 & 1.11 & 1.15 & 1.58 & 1.34 & 1.07 & 1.37 & 1.40 \\
\hline & Kupiec & 1 & 1 & 14 & 2 & 4 & 10 & 3 & 0 & 4 & 5 \\
\hline \multicolumn{12}{|c|}{ NASDAQ } \\
\hline & Vrate & $5.91 \%$ & $4.45 \%$ & $3.97 \%$ & $5.26 \%$ & $5.51 \%$ & $1.17 \%$ & $1.34 \%$ & $0.89 \%$ & $1.42 \%$ & $1.46 \%$ \\
\hline & $\begin{array}{l}\text { Vrate } \\
\text { Ratio }\end{array}$ & 1.18 & 0.89 & 0.79 & 1.05 & 1.10 & 1.17 & 1.34 & 0.89 & 1.42 & 1.46 \\
\hline & Kupiec & 4 & 2 & 6 & 0 & 1 & 1 & 3 & 0 & 4 & 5 \\
\hline
\end{tabular}

Notes: The table displays the performance of five Value-at-Risk measures, Historical Volatility (HV), GJR-Garch (GJR), Implied Volatility (IV), Non-Parametric Adjusted Implied Volatility (NPAIV), Parametric Adjusted Implied Volatility (PAIV), in term of empirical violation rate (Vrate), empirical violation rate over theoretical violation rate (VrateRatio) and the Kupiec Likelihood Ratio. The bold numbers indicate that that the null hypothesis of the two tail Kupiec is rejected at the 5\% confidence level. The results are presented for two different confidence levels (5\% and 1\%) and for three different indices: S\&P 500, Dow Jones Industrial Average and the Nasdaq. 
Table 5.7: Monthly dynamic quantile tests

\begin{tabular}{|c|c|c|c|c|c|c|c|c|c|c|}
\hline & \multicolumn{5}{|c|}{$5 \%$ VaR } & \multicolumn{5}{|c|}{$1 \%$ VaR } \\
\hline & HV & GJR & IV & NPAIV & PAIV & HV & GJR & IV & NPAIV & PAIV \\
\hline \multicolumn{11}{|l|}{ S\&P } \\
\hline \multirow[t]{3}{*}{ Constant } & & & - & & & & & - & & \\
\hline & 0.02 & -0.01 & 0.02 & 0.01 & 0.00 & 0.01 & 0.00 & 0.01 & 0.00 & 0.00 \\
\hline & 1.18 & -0.48 & 2.39 & 0.90 & 0.28 & 1.21 & -0.07 & 3.59 & 0.82 & 0.60 \\
\hline \multirow[t]{3}{*}{ Lag Hit } & & & - & & & & & & & \\
\hline & -0.04 & -0.01 & 0.02 & -0.04 & -0.03 & -0.01 & 0.00 & 0.00 & 0.00 & -0.01 \\
\hline & & & - & & & & & - & & \\
\hline \multirow{3}{*}{ Lag 2 Hit } & -2.50 & -0.49 & 0.92 & -1.51 & -1.26 & -0.97 & -0.12 & 0.26 & -0.23 & -0.68 \\
\hline & & & - & & & & & - & & \\
\hline & -0.02 & -0.03 & 0.02 & -0.03 & -0.04 & 0.00 & -0.01 & 0.01 & -0.01 & -0.01 \\
\hline \multirow{4}{*}{ Lag 3 Hit } & & & - & & & & & - & & \\
\hline & -0.99 & -2.69 & 2.28 & -2.10 & -2.97 & -1.54 & -2.33 & 1.92 & -1.89 & -3.53 \\
\hline & 0.02 & -0.01 & 0.00 & 0.00 & 0.00 & 0.01 & -0.01 & $\overline{0} .01$ & -0.01 & 0.01 \\
\hline & & & - & & & & & - & & \\
\hline \multirow{4}{*}{ Lag 4 Hit } & 0.46 & -0.52 & 0.17 & -0.10 & 0.06 & 0.67 & -2.66 & 2.08 & -2.39 & 0.46 \\
\hline & & & - & & & & & - & & \\
\hline & -0.04 & -0.04 & 0.02 & -0.04 & -0.04 & -0.01 & -0.01 & 0.01 & -0.01 & -0.01 \\
\hline & & & - & & & & & - & & \\
\hline \multirow{4}{*}{ Lag VaR } & -3.35 & -4.17 & 2.45 & -3.44 & -3.67 & -3.31 & -2.87 & 2.17 & -2.80 & -3.50 \\
\hline & & & - & & & & & - & & \\
\hline & 0.21 & 0.01 & 0.02 & 0.11 & 0.00 & 0.04 & 0.00 & 0.02 & 0.01 & 0.00 \\
\hline & & & - & & & & & - & & \\
\hline \multirow{4}{*}{$\begin{array}{l}\text { Lag Squared } \\
\text { return }\end{array}$} & 2.12 & 0.04 & 0.21 & 1.36 & 1.21 & 1.96 & -0.13 & 1.26 & 0.43 & 0.19 \\
\hline & & & - & & & & & - & & \\
\hline & 0.03 & -0.70 & 0.82 & -1.02 & -1.53 & -0.02 & -0.33 & 0.39 & -0.40 & -0.51 \\
\hline & & & - & & & & & - & & \\
\hline \multirow{4}{*}{$\begin{array}{l}\text { Lag Change } \\
\text { Vix }\end{array}$} & 0.04 & -0.58 & 0.97 & -1.22 & -2.30 & -0.08 & -1.37 & 1.59 & -1.40 & -2.31 \\
\hline & & & & & & & & & & \\
\hline & 0.02 & 0.00 & 0.00 & 0.02 & 0.04 & 0.00 & 0.00 & 0.00 & 0.00 & 0.01 \\
\hline & 0.53 & -0.12 & 0.02 & 0.51 & 0.79 & 0.19 & -0.42 & 0.33 & -0.07 & 0.58 \\
\hline \multirow{3}{*}{$\begin{array}{l}\text { Adj_R2 } \\
\text { DQoos P- } \\
\text { Value }\end{array}$} & 0.01 & 0.00 & 0.02 & 0.01 & 0.00 & 0.00 & 0.00 & 0.00 & 0.00 & 0.00 \\
\hline & & & & & & & & & & \\
\hline & 0.00 & 0.01 & 0.00 & 0.00 & 0.00 & 0.42 & 0.88 & 0.15 & 0.63 & 0.31 \\
\hline \multicolumn{11}{|l|}{ DJIA } \\
\hline \multirow[t]{2}{*}{ Constant } & 0.03 & 0.01 & 0.00 & 0.03 & 0.02 & 0.02 & 0.01 & 0.01 & 0.01 & 0.01 \\
\hline & 1.82 & 0.71 & $\overline{-}-26$ & 1.73 & 1.27 & 1.77 & 1.41 & 0.86 & 1.51 & 1.18 \\
\hline \multirow[t]{2}{*}{ Lag Hit } & -0.08 & -0.07 & $\overline{-}-05$ & -0.07 & -0.05 & -0.02 & -0.02 & $\begin{array}{l}- \\
0.01\end{array}$ & -0.01 & -0.02 \\
\hline & -3.38 & -2.61 & - & -3.11 & -1.83 & -1.82 & -1.69 & $-\overline{0.99}$ & -1.85 & -2.09 \\
\hline \multirow[t]{2}{*}{ Lag 2 Hit } & -0.01 & 0.01 & 0.01 & 0.00 & -0.03 & -0.01 & -0.01 & $\begin{array}{l}- \\
0.01\end{array}$ & -0.01 & -0.01 \\
\hline & -0.43 & 0.24 & 0.38 & -0.11 & -2.54 & -1.16 & -1.57 & $-\overline{1.59}$ & -2.24 & -2.26 \\
\hline \multirow[t]{2}{*}{ Lag 3 Hit } & 0.01 & -0.01 & $\begin{array}{l}- \\
0.01\end{array}$ & -0.02 & 0.01 & -0.01 & -0.01 & $\overline{-}$ & -0.01 & 0.01 \\
\hline & 0.23 & -0.43 & $\begin{array}{l}- \\
0.92\end{array}$ & -0.83 & 0.14 & -1.93 & -2.06 & $\overline{-}$ & -2.50 & 0.57 \\
\hline \multirow[t]{2}{*}{ Lag 4 Hit } & -0.05 & -0.02 & 0.00 & -0.04 & -0.03 & -0.01 & -0.01 & $\begin{array}{l}- \\
0.01\end{array}$ & -0.01 & -0.01 \\
\hline & -3.50 & -0.83 & $-\overline{0.12}$ & -2.77 & -1.33 & -2.89 & -2.66 & $\begin{array}{l}- \\
2.11\end{array}$ & -2.85 & -3.19 \\
\hline
\end{tabular}


Forecasting Volatility or Value-at-Risk: when does the Volatility Risk Premium matter?

\begin{tabular}{|c|c|c|c|c|c|c|c|c|c|c|}
\hline & \multicolumn{5}{|c|}{$5 \%$ VaR } & \multicolumn{5}{|c|}{$1 \% \mathrm{VaR}$} \\
\hline & HV & GJR & IV & NPAIV & PAIV & HV & GJR & IV & NPAIV & PAIV \\
\hline \multirow{2}{*}{ Lag VaR } & 0.30 & 0.15 & 0.10 & 0.18 & 0.00 & 0.07 & 0.04 & 0.03 & 0.03 & 0.00 \\
\hline & 2.47 & 1.02 & 0.64 & 1.86 & 2.03 & 1.59 & 0.98 & 0.64 & 1.34 & 1.47 \\
\hline \multirow[t]{2}{*}{$\begin{array}{l}\text { Lag Squared } \\
\text { return }\end{array}$} & -0.62 & -0.36 & $\begin{array}{l}- \\
0.65\end{array}$ & -1.74 & -2.89 & -0.49 & -0.46 & $\overline{0}-54$ & -0.64 & -0.83 \\
\hline & -0.63 & -0.28 & $\overline{0}-49$ & -1.80 & -3.52 & -1.07 & -0.91 & $\overline{-}$ & -1.50 & -1.73 \\
\hline \multirow{2}{*}{$\begin{array}{l}\text { Lag Change } \\
\text { IV }\end{array}$} & 0.08 & 0.06 & 0.04 & 0.07 & 0.09 & 0.03 & 0.03 & 0.01 & 0.01 & 0.03 \\
\hline & 1.91 & 1.25 & 0.98 & 1.59 & 1.83 & 1.48 & 1.62 & 0.84 & 1.35 & 2.01 \\
\hline Adj_R2 & 0.02 & 0.00 & 0.01 & 0.01 & 0.01 & 0.01 & 0.00 & 0.00 & 0.00 & 0.00 \\
\hline $\begin{array}{l}\text { DQoos P- } \\
\text { Value }\end{array}$ & 0.00 & 0.01 & 0.01 & 0.00 & 0.00 & 0.00 & 0.01 & 0.56 & 0.03 & 0.00 \\
\hline \multicolumn{11}{|l|}{ NASDAQ } \\
\hline \multirow[t]{2}{*}{ Constant } & 0.04 & 0.00 & 0.00 & 0.01 & 0.01 & 0.01 & 0.00 & 0.00 & 0.01 & 0.00 \\
\hline & 1.73 & -0.16 & $-\overline{0.06}$ & 0.90 & 0.71 & 0.72 & 0.38 & $-\overline{0.71}$ & 0.83 & 0.37 \\
\hline \multirow[t]{2}{*}{ Lag Hit } & 0.01 & 0.04 & 0.07 & 0.04 & 0.00 & -0.01 & -0.01 & $\overline{0} .01$ & -0.01 & -0.08 \\
\hline & 0.29 & 1.03 & 1.21 & 0.79 & -0.01 & -0.71 & -0.57 & $\overline{-}-50$ & -0.39 & -1.16 \\
\hline \multirow[t]{2}{*}{ Lag 2 Hit } & -0.01 & -0.02 & $\overline{-}-03$ & -0.05 & -0.02 & -0.01 & -0.02 & $\begin{array}{l}- \\
0.01\end{array}$ & -0.02 & -0.01 \\
\hline & -0.62 & -0.92 & - & -2.26 & -0.99 & -0.49 & -1.43 & - & -1.34 & -1.46 \\
\hline \multirow[t]{2}{*}{ Lag 3 Hit } & 0.04 & 0.02 & $\overline{-}$ & 0.02 & 0.02 & -0.01 & -0.02 & $-\overline{0}$ & -0.02 & -0.01 \\
\hline & 0.88 & 0.52 & - & 0.50 & 0.67 & -0.72 & -1.54 & - & -1.36 & -1.72 \\
\hline \multirow[t]{2}{*}{ Lag 4 Hit } & -0.03 & -0.05 & - & -0.05 & -0.06 & -0.01 & -0.02 & - & -0.02 & -0.02 \\
\hline & -1.35 & -3.06 & - & -3.32 & -4.09 & -1.51 & -1.91 & - & -1.82 & -2.38 \\
\hline \multirow[t]{2}{*}{ Lag VaR } & 0.28 & 0.02 & 0.08 & 0.11 & 0.04 & 0.03 & 0.00 & - & 0.01 & 0.01 \\
\hline & 2.08 & 0.10 & 0.61 & 0.97 & 2.53 & 0.98 & -0.02 & $\overline{0} .31$ & 0.14 & 1.72 \\
\hline \multirow[t]{2}{*}{$\begin{array}{l}\text { Lag Squared } \\
\text { return }\end{array}$} & 1.47 & 0.35 & 0.55 & 0.57 & 0.86 & 0.25 & 0.42 & 0.30 & 0.21 & 1.34 \\
\hline & 1.44 & 0.36 & 0.65 & 0.69 & 0.80 & 0.84 & 0.91 & 0.91 & 0.73 & 1.41 \\
\hline \multirow[t]{2}{*}{$\begin{array}{l}\text { Lag Change } \\
\text { IV }\end{array}$} & -0.04 & -0.07 & - & -0.06 & 0.02 & 0.00 & -0.02 & $-\overline{0.02}$ & -0.01 & 0.03 \\
\hline & -1.01 & -1.76 & - & -1.49 & 0.50 & 0.05 & -0.98 & - & -0.96 & 0.87 \\
\hline Adj_R2 & 0.01 & 0.00 & 0.01 & 0.01 & 0.01 & 0.00 & 0.00 & 0.00 & 0.00 & 0.01 \\
\hline $\begin{array}{l}\text { DQoos P- } \\
\text { Value }\end{array}$ & 0.00 & 0.04 & 0.01 & 0.00 & 0.00 & 0.84 & 0.29 & 0.88 & 0.23 & 0.00 \\
\hline
\end{tabular}


Table 5.8: Monthly loss function and loss function difference tests

\begin{tabular}{|c|c|c|c|c|c|c|c|c|c|c|c|}
\hline \multirow{2}{*}{ S\&P } & \multicolumn{5}{|c|}{$5 \%$ VaR } & \multicolumn{6}{|c|}{$1 \%$ VaR } \\
\hline & HV & GJR & IV & NPAIV & PAIV & & HV & GJR & IV & NPAIV & PAIV \\
\hline $\begin{array}{l}\text { Loss } \\
\text { Function }\end{array}$ & 28.43 & 26.65 & 28.12 & 28.42 & 64.82 & & 7.99 & 7.54 & 7.98 & 8.02 & 20.21 \\
\hline \multicolumn{12}{|c|}{$\begin{array}{l}\text { Loss Function } \\
\text { Differences }\end{array}$} \\
\hline HV & 0.00 & 1.82 & 0.34 & 0.03 & $\begin{array}{l}\overline{-} \\
36.22\end{array}$ & HV & 0.00 & 0.46 & 0.01 & -0.03 & $\overline{12.19}$ \\
\hline GJR & -1.82 & 0.00 & -1.48 & -1.79 & $\begin{array}{l}- \\
38.04\end{array}$ & GJR & -0.46 & 0.00 & -0.45 & -0.49 & $-\overline{12.65}$ \\
\hline IV & -0.34 & 1.48 & 0.00 & -0.31 & 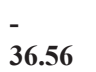 & IV & -0.01 & 0.45 & 0.00 & -0.04 & $-\overline{12.20}$ \\
\hline NPAIV & -0.03 & 1.79 & 0.31 & 0.00 & $-\overline{36.25}$ & NPAIV & 0.03 & 0.49 & 0.04 & 0.00 & $-\overline{12.16}$ \\
\hline PAIV & 36.22 & 38.04 & 36.56 & 36.25 & 0.00 & PAIV & 12.19 & 12.65 & 12.20 & 12.16 & 0.00 \\
\hline DJIA & HV & GJR & IV & NPAIV & PAIV & & HV & GJR & IV & NPAIV & PAIV \\
\hline $\begin{array}{l}\text { Loss } \\
\text { Function }\end{array}$ & 19.82 & 18.69 & 18.92 & 19.22 & 26.81 & & 5.61 & 5.27 & 5.26 & 5.44 & 7.45 \\
\hline
\end{tabular}

Loss Function

Differences

\begin{tabular}{|c|c|c|c|c|c|c|c|c|c|c|c|}
\hline & HV & GJR & IV & NPAIV & PAIV & & HV & GJR & IV & NPAIV & PAIV \\
\hline HV & 0.00 & 1.13 & 0.92 & 0.60 & -6.94 & HV & 0.00 & 0.35 & 0.36 & 0.17 & $\begin{array}{l}-1.83 \\
\end{array}$ \\
\hline GJR & -1.13 & 0.00 & -0.22 & -0.53 & -8.07 & GJR & -0.35 & 0.00 & 0.02 & -0.18 & -2.18 \\
\hline IV & -0.92 & 0.22 & 0.00 & -0.32 & -7.85 & IV & -0.36 & -0.02 & 0.00 & -0.20 & -2.19 \\
\hline NPAIV & -0.60 & 0.53 & 0.32 & 0.00 & -7.54 & NPAIV & -0.17 & 0.18 & 0.20 & 0.00 & -2.00 \\
\hline PAIV & 6.94 & 8.07 & 7.85 & 7.54 & 0.00 & PAIV & 1.83 & 2.18 & 2.19 & 2.00 & 0.00 \\
\hline \multicolumn{12}{|l|}{ NASDAQ } \\
\hline & HV & GJR & IV & NPAIV & PAIV & & HV & GJR & IV & NPAIV & PAIV \\
\hline $\begin{array}{l}\text { Loss } \\
\text { Function }\end{array}$ & 17.42 & 16.59 & 17.60 & 17.52 & 21.24 & & 4.82 & 4.48 & 4.51 & 4.55 & 6.27 \\
\hline \multicolumn{12}{|c|}{$\begin{array}{l}\text { Loss Function } \\
\text { Differences }\end{array}$} \\
\hline & HV & GJR & IV & NPAIV & PAIV & & $\mathrm{HV}$ & GJR & IV & NPAIV & PAIV \\
\hline $\mathrm{HV}$ & 0.00 & 0.83 & -0.18 & -0.11 & -3.85 & $\mathrm{HV}$ & 0.00 & 0.34 & 0.31 & 0.28 & -1.46 \\
\hline GJR & -0.83 & 0.00 & -1.01 & -0.94 & -4.68 & GJR & -0.34 & 0.00 & -0.03 & -0.06 & -1.80 \\
\hline IV & 0.18 & 1.01 & 0.00 & 0.08 & -3.67 & IV & -0.31 & 0.03 & 0.00 & -0.03 & -1.77 \\
\hline NPAIV & 0.11 & 0.94 & -0.08 & 0.00 & -3.75 & NPAIV & -0.28 & 0.06 & 0.03 & 0.00 & -1.73 \\
\hline PAIV & 3.85 & 4.68 & 3.67 & 3.75 & 0.00 & PAIV & 1.46 & 1.80 & 1.77 & 1.73 & 0.00 \\
\hline
\end{tabular}

Notes: The table displays the value of the loss functions for the five VaR measures over three indices. The smaller the loss function, the better the VaR measure. In order to compare the loss function across models, we compute the difference between the loss functions provided by a model on the vertical column and the loss function of the horizontal column. A block bootstrap is performed in order to test for that statistical significance of the loss function difference. The block length is chosen according to the following rule of thumb (numberofobservations) $)^{1 / 3}$. We rely on 1,000 bootstraps.

Our findings show that predicting volatility and predicting a certain quantile are two different objective functions. As it is the case for IV, the optimal forecast for volatility does not necessarily translate into the optimal forecast for a quantile. Giot and Laurent (2004) reach a similar conclusion when assessing the performance of intra-day data based VaR forecasts. 
Two factors can explain why IV is the best source of information for predicting volatility but not for predicting quantiles.

The relative predictive power of volatility forecasts is sensitive to the precision of the volatility measure used. IV is found to be a good forecast on standard volatility proxies. But Martin et al. (2009) re-assess this finding using a set of noise robust volatility measures. They conclude that IV does not outperform other models in predicting S\&P 500 volatility. Besides, simple Garch models forecast intraday volatility very accurately (Andersen and Bollerslev, 1998). Quantile prediction requires a greater degree of precision, which explains the disappointing results of IV information to forecast VaR.

The implicit (Prokopczuk and Wise Simen, 2014) or explicit (Jorion, 1995) volatility risk premium adjustments in the volatility forecasting literature always relies on important assumptions. The volatility risk premium is only linearly dependent on the volatility level and independent from returns. These assumptions are sufficient for forecasting the average volatility level over time. Our results suggest that they are insufficient for forecasting extreme quantiles where non-linearity and return dependence matter. Supporting this conjecture, Charoenwong et al. (2009) show that IV was unable to provide better volatility forecasts than historical volatility models during high volatility regimes. Additionally, the dynamic quantile test performed in this paper demonstrates that the adjustment allows correcting for the average level of violations but leads to an increased probability of violation during high volatility regimes. More complex dynamics of the volatility risk premium are necessary to adjust IV for VaR forecasting purposes.

Overall, we find IV has limited utility for predicting quantiles of future returns and for predicting VaR. The volatility risk premium adjustment that is proved to be performance enhancing in volatility forecasting (Prokopczuk and Wise Simen, 2014) or portfolio allocation (DeMiguel et al., 2013) is not so for VaR forecasting. This exercise underlines the difference between volatility forecasting and quantile forecasting.

\subsection{CONCLUSION}

This paper sheds new light on the comparison between option implied volatility and historical returns time series information. The efficiency of these two sources of information has been evaluated and compared at numerous occasions based on their predictive power of future realized volatility. We proposed to re-evaluate the relative benefits of these two information sources with a more pragmatic objective. Which out of IV and historical volatility models provides the best Value-at-Risk estimate?

We also formally proposed to correct the IV for the volatility risk premium. IV estimates are upward biased and are found to lead to overstated VaR because of the negative price of 


\section{CHAPTER 5}

volatility risk embedded in options. Our non-parametric and parametric adjustments for the volatility risk premium reduce this bias and formally account for the presence of the volatility risk premium. Previous studies mainly ignore the volatility risk premium or account for it in an indirect and unconscious way.

The results for three different indices and using different evaluation criteria all point in the same direction. IV VaRs do not outperform historical volatility model VaRs. A simple VaR based on a Garch accounting for leverage provides very satisfactory results. IV VaR, even after volatility risk premium adjustment, cannot outperform this Garch VaR. In fact, it even appears that on many occasions the Garch VaR outperforms most of its competitors. Hence the conclusion of the superiority of the IV over the historical volatility model cannot be easily transposed to the risk management context of the VaR measure. 


\section{References}

Allende, H., Elías, C., \& Torres, S. (2004). Estimation of the option prime: Microsimulation of backward stochastic differential equations. International statistical review,72(1), 107-121.

Amin, K., Coval, J. D., \& Seyhun, H. N. (2004). Index Option Prices and Stock Market Momentum. The Journal of Business, 77(4), 835-874.

Andersen, T. G., \& Bollerslev, T. (1998). Answering the skeptics: Yes, standard volatility models do provide accurate forecasts. International Economic Review, 885-905.

Anderson, E. W., Ghysels, E., \& Juergens, J. L. (2005). Do heterogeneous beliefs matter for asset pricing?. Review of Financial Studies, 18(3), 875-924.

Anderson, E. W., Ghysels, E., \& Juergens, J.L. (2009) The impact of risk and uncertainty on expected returns.Journal of Financial Economics, 94(2), 233-263.

Ang, A., Hodrick, R. J., Xing, Y., \& Zhang, X. (2006). The cross-section of volatility and expected returns. The Journal of Finance, 61(1), 259-299.

Aristotle, Jowett, B., \& Davis, H. W. C. (1920). Aristotle's Politics. Oxford: At the Clarendon Press.

Atılgan, Y. (2010). Deviations from put-call parity and earnings announcement returns. Journal of Financial and Quantitative Analysis.

Baele, L., Bekaert, G., \& Inghelbrecht, K. (2010). The determinants of stock and bond return comovements. Review of Financial Studies, 23(6), 2374-2428.

Baker, S. R., Bloom, N., \& Davis, S. J. (2012). Measuring economic policy uncertainty. policyuncertainy.com.

Bakshi, G., Cao, C., \& Chen, Z. (1997). Empirical performance of alternative option pricing models. The Journal of Finance, 52(5), 2003-2049.

Bakshi, G., Kapadia, N., \& Madan, D. (2003). Stock return characteristics, skew laws, and the differential pricing of individual equity options. Review of Financial Studies, 16(1), 101-143.

Bali, T., \& Zhou, H. (2012). Risk, uncertainty, and expected returns. Available at SSRN 2020604.

Bams, D., Lehnert, T., \& Wolff, C. C. (2005). An evaluation framework for alternative VaR-models. Journal of International Money and Finance, 24(6), 944-958.

Bams, D., Lehnert, T., \& Wolff, C. C. (2009). Loss functions in option valuation: a framework for selection. Management Science, 55(5), 853-862.

Banerjee, N. (2002, June 25). For OPEC, Watchword Is Wait and See. The New York Times. Pg. 2.

Bansal, R., \& Yaron, A. (2004). Risks for the long run: A potential resolution of asset pricing puzzles. The Journal of Finance, 59(4), 1481-1509.

Barle, S., \& Cakici, N. (1995). Growing a smiling tree. Risk, 8(10), 76-81. 


\section{REFERENCES}

Barone-Adesi, G,. \& Whaley, R. E. (1987). Efficient analytic approximation of American option values. The Journal of Finance, 42(2), 301-320.

Barone-Adesi, G., Bourgoin, F., \& Giannopoulos, K. (1998). Market Risk: Don't Look Back. Risk, 11, 100-103.

Barone-Adesi, G., Engle, R. F., \& Mancini, L. (2008). A GARCH option pricing model with filtered historical simulation. Review of Financial Studies, 21(3), 1223-1258.

Bartram, S. M., Brown, G. W., \& Conrad, J. (2011). The effects of derivatives on firm risk and value. Journal of Financial and Quantitative Analysis, 46(04), 967-999.

Baur, D. G., \& McDermott, T. K. (2010). Is gold a safe haven? International evidence. Journal of Banking \& Finance, 34(8), 1886-1898.

Beckers, S. (1981). Standard deviations implied in option prices as predictors of future stock price variability. Journal of Banking \& Finance, 5(3), 363-381.

Bekaert, G., Engstrom, E., \& Xing, Y. (2009). Risk, uncertainty, and asset prices. Journal of Financial Economics, 91(1), 59-82.

Ben-Rephael, A., Kadan, O., \& Wohl, A. (2008). The diminishing liquidity premium (No. 2008/52). CFS Working Paper.

Berkowitz, J. (2010). On justifications for the ad hoc Black-Scholes method of option pricing. Studies in Nonlinear Dynamics \& Econometrics, 14(1).

Berkowitz, J., \& O’Brien, J. (2002). How Accurate Are Value-at-Risk Models at Commercial Banks?. The Journal of Finance, 57(3), 1093-1111.

Bernstein, P. L., \& Bernstein Peter, L. (1996). Against the gods: The remarkable story of risk. New York: Wiley.

Black, F., \& Scholes, M. (1973). The pricing of options and corporate liabilities. The journal of political economy, 637-654.

Blair, B. J., Poon, S. H., \& Taylor, S. J. (2010). Forecasting S\&P 100 volatility: the incremental information content of implied volatilities and high-frequency index returns. Journal of Econometrics, 105(1), 5-26.

Bollen, N. P., \& Whaley, R. E. (2004). Does net buying pressure affect the shape of implied volatility functions?. The Journal of Finance, 59(2), 711-753.

Bollerslev, T., \& Ole Mikkelsen, H. (1999). Long-term equity anticipation securities and stock market volatility dynamics. Journal of Econometrics,92(1), 75-99.

Bollerslev, T., Gibson, M., \& Zhou, H. (2011). Dynamic estimation of volatility risk premia and investor risk aversion from option-implied and realized volatilities. Journal of Econometrics, 160(1), 235-245.

Borgers, A., Derwall, J., Koedijk, K., \& Ter Horst, J. (2014). New insights into the ESG performance and financial performance of mutual funds. ECCE webinar: June 25

Brandt, M. W., \& Wu, T. (2002). Cross-sectional tests of deterministic volatility functions. Journal of Empirical Finance, 9(5), 525-550.

Britten-Jones, M., \& Neuberger, A. (2000). Option prices, implied price processes, and stochastic volatility. The Journal of Finance, 55(2), 839-866.

Broadie, M., Chernov, M., \& Johannes, M. (2007). Model specification and risk premia: Evidence from futures options. The Journal of Finance, 62(3), 1453-1490.

Buraschi, A., Trojani, F., \& Vedolin, A. (2013). Economic uncertainty, disagreement, and credit markets. Management Science. 
Buraschi, A., Trojani, F., \& Vedolin, A. (2014). When uncertainty blows in the orchard: Comovement and equilibrium volatility risk premia. The Journal of Finance, 69(1), 101-137.

Canina, L., \& Figlewski, S. (1993). The informational content of implied volatility. Review of Financial Studies, 6(3), 659-681.

Capelle-Blancard, G. (2011). Are derivatives dangerous? A literature survey.Economie internationale, (3), 6789.

Carhart, M. M. (1997). On persistence in mutual fund performance. The Journal of Finance, 52(1), 57-82.

Carr, P. \& Wu, L. (2009). Variance risk premiums. Review of Financial Studies, 22, 1311-1341.

Cavaglia, S., Brightman, C., \& Aked, M. (2000). The increasing importance of industry factors. Financial Analysts Journal, 41-54.

Chan, K. F., Treepongkaruna, S., Brooks, R., \& Gray, S. (2011). Asset market linkages: Evidence from financial, commodity and real estate assets. Journal of Banking \& Finance, 35(6), 1415-1426.

Chang, B. Y., Christoffersen, P., \& Jacobs, K. (2013). Market skewness risk and the cross section of stock returns. Journal of Financial Economics, 107(1), 46-68.

Charoenwong, C., Jenwittayaroje, N., \& Low, B. S. (2009). Who knows more about future currency volatility?. Journal of Futures Markets, 29(3), 270-295.

Chen, N. F., Roll, R., \& Ross, S. A. (1986). Economic forces and the stock market. Journal of business, 59(3), 383.

Chen, Q., \& Gerlach, R. H. (2013). The two-sided Weibull distribution and forecasting financial tail risk. International Journal of Forecasting, 29(4), 527-540.

Cheng, X., \& Fung, J. K. (2012). The Information Content of Model-Free Implied Volatility. Journal of Futures Markets, 32(8), 792-806.

Chernov, M. (2007). On the role of risk premia in volatility forecasting. Journal of Business \& Economic Statistics, 25(4), 411-426.

Chernov, M., Ronald Gallant, A., Ghysels, E., \& Tauchen, G. (2003). Alternative models for stock price dynamics. Journal of Econometrics, 116(1), 225-257.

Chiras, D. P., \& Manaster, S. (1978). The information content of option prices and a test of market efficiency. Journal of Financial Economics, 6(2), 213-234.

Christensen, B. J., \& Prabhala, N. R. (1998). The relation between implied and realized volatility. Journal of Financial Economics, 50(2), 125-150.

Christoffersen, P., \& Jacobs, K. (2004a). Which GARCH model for option valuation?. Management Science, 50(9), 1204-1221.

Christoffersen, P., \& Jacobs, K. (2004b). The importance of the loss function in option valuation. Journal of Financial Economics, 72(2), 291-318.

Christoffersen, P., Heston, S., \& Jacobs, K. (2013). Capturing option anomalies with a variance-dependent pricing kernel. Review of Financial Studies, 26(8), 1963-2006

Cohen, L., \& Frazzini, A. (2008). Economic links and predictable returns. The Journal of Finance, 63(4), 19772011.

Connolly, R., Stivers, C., \& Sun, L. (2005). Stock market uncertainty and the stock-bond return relation. Journal of Financial and Quantitative Analysis, 40(01), 161-194. 


\section{REFERENCES}

Cornaggia, J. (2013). Does risk management matter? Evidence from the US agricultural industry. Journal of Financial Economics, 109(2), 419-440.

Corrado, C. J., \& Miller Jr, T. W. (2005). The forecast quality of CBOE implied volatility indexes. Journal of Futures Markets, 25(4), 339-373.

Covello, V. T., \& Mumpower, J. (1985). Risk analysis and risk management: an historical perspective. Risk analysis, 5(2), 103-120.

Cremers, M., \& Weinbaum, D. (2010). Deviations from put-call parity and stock return predictability. Journal of Financial and Quantitative Analysis, 45(2), 335.

Cummins, J. D., Phillips, R. D., \& Smith, S. D. (1998). The rise of risk management. Economic Review-Federal Reserve Bank of Atlanta, 83, 30-41.

David, A., \& Veronesi, P. (2008). Inflation and earnings uncertainty and volatility forecasts: A structural form approach. Chicago GSB Research Paper.

Day, T. E., \& Lewis, C. M. (1992). Stock market volatility and the information content of stock index options. Journal of Econometrics, 52(1), 267-287.

DellaVigna, S., \& Pollet, J. M. (2009). Investor inattention and Friday earnings announcements. The Journal of Finance, 64(2), 709-749.

DeMiguel, V., Plyakha, Y., Uppal, R., \& Vilkov, G. (2013). Improving portfolio selection using option-implied volatility and skewness. Journal of Financial and Quantitative Analysis, 48(06), 1813-1845.

Diebold, F. X. (2012). Comparing predictive accuracy, twenty years later: A personal perspective on the use and abuse of diebold-mariano tests (No. w18391). National Bureau of Economic Research.

Diebold, F. X., \& Mariano, R. S. (1995). Comparing predictive accuracy. Journal of Business \& economic statistics, 13, 253-263

Dimson, E., \& Mussavian, M. (1999). Three centuries of asset pricing. Journal of Banking \& Finance, 23(12), 1745-1769.

Driesprong, G., Jacobsen, B., \& Maat, B. (2008). Striking oil: Another puzzle?Journal of Financial Economics, 89(2), 307-327.

Duan, J. C. (1995). The GARCH option pricing model. Mathematical finance,5(1), 13-32.

Duan, J. C. (1996). Cracking the smile. Risk, 9(12), 55-59.

Duan, J. C., \& Simonato, J. G. (1998). Empirical martingale simulation for asset prices. Management Science, 44(9), 1218-1233

Dumas, B., Fleming, J., \& Whaley, R. E. (1998). Implied volatility functions: Empirical tests. The Journal of Finance, 53(6), 2059-2106.

Easley, D., O'hara, M., \& Srinivas, P. S. (1998). Option volume and stock prices: Evidence on where informed traders trade. The Journal of Finance,53(2), 431-465.

Ederington, L. H., \& Guan, G. (2002). Is Implied Volatility an Informationally Efficient and Effective Predictor of Future Volatility?. Journal of Risk, 4, 29-46.

Elder, J., Miao, H., \& Ramchander, S. (2012). Impact of macroeconomic news on metal futures. Journal of Banking \& Finance, 36(1), 51-65.

Ellsberg, D. (1961). Risk, Ambiguity and the Savage Axioms. The Quarterly Journal of Economics, 75, 643669. 
Engle, R. F., \& Manganelli, S. (2004). CAViaR: Conditional autoregressive value at risk by regression quantiles. Journal of Business \& Economic Statistics,22(4), 367-381.

Engle, R. F., \& Mustafa, C. (1992). Implied ARCH models from options prices. Journal of Econometrics, 52(1), 289-311.

Engle, R. F., \& Ng, V. K. (1993). Measuring and testing the impact of news on volatility. The Journal of Finance, 48(5), 1749-1778.

Fama, E. F., \& French, K. R. (1993). Common risk factors in the returns on stocks and bonds. Journal of Financial Economics, 33(1), 3-56.

Ferson, W. E., \& Harvey, C. R. (1993). The risk and predictability of international equity returns. Review of Financial Studies, 6(3), 527-566.

Fleming, J. (1998). The quality of market volatility forecasts implied by S\&P 100 index option prices. Journal of Empirical Finance, 5(4), 317-345.

Frijns, B., Lehnert, T., \& Zwinkels, R. C. (2010). Behavioral heterogeneity in the option market. Journal of Economic Dynamics and Control, 34(11), 2273-2287.

Frijns, B., Tallau, C., \& Tourani-Rad, A. (2010). The information content of implied volatility: evidence from Australia. Journal of Futures Markets, 30(2), 134-155.

Giot, P. (2005). Implied volatility indexes and daily Value at Risk models. The Journal of Derivatives, 12(4), 5464.

Giot, P., \& Laurent, S. (2003). Value-at-risk for long and short trading positions.Journal of Applied Econometrics, 18(6), 641-663.

Giot, P., \& Laurent, S. (2004). Modelling daily value-at-risk using realized volatility and ARCH type models. Journal of Empirical Finance, 11(3), 379-398.

Glosten, L. R., Jagannathan, R., \& Runkle, D. E. (1993). On the relation between the expected value and the volatility of the nominal excess return on stocks. The Journal of Finance, 48(5), 1779-1801.

Gurkaynak, R., \& Wolfers, J. (2006). Macroeconomic derivatives: An initial analysis of market-based macro forecasts, uncertainty, and risk (No. w11929). National Bureau of Economic Research.

Hamilton, J. D. (1983). Oil and the macroeconomy since World War II. The Journal of Political Economy, 228248.

Hansen, P. R., \& Lunde, A. (2005). A forecast comparison of volatility models: does anything beat a GARCH (1, 1)?. Journal of Applied Econometrics, 20(7), 873-889.

Hansen, P. R., Lunde, A., \& Nason, J. M. (2011). The model confidence set. Econometrica, 79(2), 453-497.

Hao, J., \& Zhang, J. E. (2013). GARCH Option Pricing Models, the CBOE VIX, and Variance Risk Premium. Journal of Financial Econometrics, 11(3), 556-580.

Härdle, W., \& Hafner, C. M. (2000). Discrete time option pricing with flexible volatility estimation. Finance and Stochastics, 4(2), 189-207.

Harvey, C. R., \& Siddique, A. (2000). Conditional skewness in asset pricing tests. The Journal of Finance, 55(3), 1263-1295.

Henkel, S. J., Martin, J. S., \& Nardari, F. (2011). Time-varying short-horizon predictability. Journal of Financial Economics, 99(3), 560-580. 


\section{REFERENCES}

Hentschel, L. (1995). All in the family nesting symmetric and asymmetric garch models. Journal of Financial Economics, 39(1), 71-104.

Heston, S. L. (1993). A closed-form solution for options with stochastic volatility with applications to bond and currency options. Review of financial studies,6(2), 327-343.

Heston, S. L., \& Nandi, S. (2000). A closed-form GARCH option valuation model. Review of Financial Studies, 13(3), 585-625.

Heston, S. L., Rouwenhorst, K. G., \& Wessels, R. E. (1995). The structure of international stock returns and the integration of capital markets. Journal of Empirical Finance, 2(3), 173-197.

Hong, H., \& Stein, J. C. (2007). Disagreement and the stock market. The Journal of Economic Perspectives, 109-128.

Hong, H., Torous, W., \& Valkanov, R. (2007). Do industries lead stock markets?. Journal of Financial Economics, 83(2), 367-396.

Hou, K., Karolyi, G. A., \& Kho, B. C. (2011). What factors drive global stock returns?. Review of Financial Studies, 24(8), 2527-2574.

Hsieh, K. C., \& Ritchken, P. (2005). An empirical comparison of GARCH option pricing models. Review of Derivatives Research, 8(3), 129-150.

Hull, J., \& Suo, W. (2002). A methodology for assessing model risk and its application to the implied volatility function model. Journal of Financial and Quantitative Analysis, 37(2), 297-318.

Jiang, G. J., \& Tian, Y. S. (2005). The model-free implied volatility and its information content. Review of Financial Studies, 18(4), 1305-1342.

Jones, C. M., \& Kaul, G. (1996). Oil and the stock markets. The Journal of Finance, 51(2), 463-491.

Jorion, P. (1995). Predicting volatility in the foreign exchange market. The Journal of Finance, 50(2), 507-528.

Kanniainen, J. (2013). Estimating and Using GARCH Models with VIX Data for Option Valuation. Journal of Banking \& Finance, 43, 200-211.

Kilian, L., \& Park, C. (2009). The impact of oil price shocks on the us stock market. International Economic Review, 50(4), 1267-1287.

Knight, F. H. (2012). Risk, uncertainty and profit. Courier Dover Publications.

Koenker, R., \& Bassett Jr, G. (1978). Regression quantiles. Econometrica: Journal of the Econometric Society, $33-50$.

Kuester, K., Mittnik, S., \& Paolella, M. S. (2006). Value-at-risk prediction: A comparison of alternative strategies. Journal of Financial Econometrics, 4(1), 53-89.

Kupiec, P. H. (1995). Techniques for verifying the accuracy of risk measurement models. Journal of Derivatives 3(2), 73-84

Lamoureux, C. G., \& Lastrapes, W. D. (1993). Forecasting stock-return variance: Toward an understanding of stochastic implied volatilities. Review of Financial Studies, 6(2), 293-326.

Ledoit, O., \& Wolf, M. (2008). Robust performance hypothesis testing with the Sharpe ratio. Journal of Empirical Finance, 15(5), 850-859.

Lehar, A., Scheicher, M., \& Schittenkopf, C. (2002). GARCH vs. stochastic volatility: Option pricing and risk management. Journal of Banking \& Finance,26(2), 323-345. 
Lehnert, T. (2003). Explaining smiles: GARCH option pricing with conditional leptokurtosis and skewness. TheJournal of Derivatives, 10(3), 27-39.

Lewellen, J., \& Nagel, S. (2006). The conditional CAPM does not explain asset-pricing anomalies. Journal of Financial Economics, 82(2), 289-314.

Makropoulou, V., \& Markellos, R. N. (2005). What is the Fair Rent Thales Should Have Paid?. HERCMA Proceedings for.

Martens, M., \& Zein, J. (2004). Predicting financial volatility: High-frequency time-series forecasts vis-à-vis implied volatility. Journal of Futures Markets,24(11), 1005-1028.

Martin, G. M., Reidy, A., \& Wright, J. (2009). Does the option market produce superior forecasts of noisecorrected volatility measures?. Journal of Applied Econometrics, 24(1), 77-104.

Menzly, L., \& Ozbas, O. (2010). Market Segmentation and Cross-predictability of Returns. The Journal of Finance, 65(4), 1555-1580.

Mittnik, S., \& Paolella, M. S. (2000). Conditional density and value-at-risk prediction of Asian currency exchange rates. Journal of Forecasting, 19(4), 313-333.

Mixon, S. (2009). Option markets and implied volatility: Past versus present.Journal of Financial Economics, 94(2), 171-191.

Narayan, P. K., \& Sharma, S. S. (2011). New evidence on oil price and firm returns. Journal of Banking \& Finance, 35(12), 3253-3262.

Neumann, M., \& Skiadopoulos, G. (2012). Predictable dynamics in higher order risk-neutral moments: Evidence from the S\&P 500 options. Journal of Financial and Quantitative Analysis (JFQA), Forthcoming.

Pan, J. (2002). The jump-risk premia implicit in options: Evidence from an integrated time-series study. Journal of financial economics, 63(1), 3-50.

Pan, J. (2002). The jump-risk premia implicit in options: Evidence from an integrated time-series study. Journal of financial economics, 63(1), 3-50.

Pérez-González, F., \& Yun, H. (2013). Risk management and firm value: evidence from weather derivatives. The Journal of Finance, 68(5), 2143-2176.

Pollet, J. M. (2005). Predicting asset returns with expected oil price changes.Available at SSRN 722201.

Pong, S., Shackleton, M. B., Taylor, S. J., \& Xu, X. (2004). Forecasting currency volatility: A comparison of implied volatilities and AR (FI) MA models.Journal of Banking \& Finance, 28(10), 2541-2563.

Prokopczuk, M., \& Simen, C. W. (2013). Variance risk premia in commodity markets. Variance Risk Premia in Commodity Markets (January 2, 2013).

Prokopczuk, M., \& Wese Simen, C. (2014). The importance of the volatility risk premium for volatility forecasting. Journal of Banking \& Finance, 40, 303-320.

Qian, Y., \& Yu, X. (2009). Business Cycle, Firm Size and Market Reactions to News. Firm Size and Market Reactions to News (July 30, 2009).

Rapach, D. E., Strauss, J. K., \& Zhou, G. (2010). Out-of-sample equity premium prediction: Combination forecasts and links to the real economy. Review of Financial Studies, 23(2), 821-862.

Rosenberg, J. V., \& Engle, R. F. (2002). Empirical pricing kernels. Journal of Financial Economics, 64(3), 341372.

Stein, J. (1989). Overreactions in the options market. The Journal of Finance,44(4), 1011-1023. 


\section{REFERENCES}

Szakmary, A., Ors, E., Kyoung Kim, J., \& Davidson III, W. N. (2003). The predictive power of implied volatility: Evidence from 35 futures markets. Journal of Banking \& Finance, 27(11), 2151-2175.

Taylor, S. J., \& Xu, X. (1997). The incremental volatility information in one million foreign exchange quotations. Journal of Empirical Finance, 4(4), 317-340.

Taylor, S. J., Yadav, P. K., \& Zhang, Y. (2010). The information content of implied volatilities and model-free volatility expectations: Evidence from options written on individual stocks. Journal of Banking \& Finance, 34(4), 871-881.

Todorov, V. (2009). Variance risk-premium dynamics: The role of jumps. Review of Financial Studies, 23, 345383.

Trolle, A., \& Schwartz, E. S. (2010). Variance risk premia in energy commodities. Journal of Derivatives, 18, 118.

Tsiaras, L. (2009). The forecast performance of competing implied volatility measures: The case of individual stocks (No. F-2009-02). University of Aarhus, Aarhus School of Business, Department of Business Studies.

Veronesi, P. (1999). Stock market overreactions to bad news in good times: a rational expectations equilibrium model. Review of Financial Studies, 12(5), 975-1007.

Wagner, D., Ellis, D. M., \& Dubofsky, D. A. (1996). The Factors Behind Put-Call Parity Violations of S\&P 100 Index Options. Financial Review, 31(3), 535-552.

Yatchew, A., \& Härdle, W. (2006). Nonparametric state price density estimation using constrained least squares and the bootstrap. Journal of Econometrics, 133(2), 579-599.

Yu, W. W., Lui, E. C., \& Wang, J. W. (2010). The predictive power of the implied volatility of options traded OTC and on exchanges. Journal of Banking \& Finance, 34(1), 1-11. 


\section{Summary}

\section{Option markets and option implied information}

Options and derivatives are powerful tools for market participants in order to manage risks or to speculate. The sizeable growth of derivative markets attests to the importance of options in the modern financial landscape. Although options are not a novel intellectual concept, the development of liquid option markets is recent. According to the Bank of International Settlement the Over-The-Counter derivatives total gross market value was marginally over $\$ 2,500$ billion in 1998. In 2013 this gross market value reached more than $\$ 18,500$ billion. This is an almost six fold increase in just fifteen years.

In addition to option traders and risk professionals, financial economists have a strong interest in option and derivative markets. This interest explains the development of many articles and dedicated journals on the topic. Options offer economists the opportunity to uncovering investors' expectations. The price of an option depends on its payoff and therefore on the expected distribution of the underlying asset (e.g. stock price, commodity price, interest rate, etc.). Accordingly, option prices contain information on investor's expectations. This valuable information is called 'option implied information'.

In this dissertation I intend to investigate, under option implied information and option implied volatility from different angles. My findings summarised in the following paragraphs highlight the opportunities and limitations arising from option implied information and from the discrepancy between the risk neutral and physical expectations. Option prices reflect both the expectation and risk preference of option traders. Because of the second component the information extracted from option prices is risk adjusted and is different from the actual physical expectations.

\section{Estimation of volatility model on option prices}

Volatility and the volatility dynamics are paramount for option pricing. The significant amount of research carried out on time series volatility modelling generates important and significant knowledge on the features of financial markets volatility. Volatility models are traditionally estimated and evaluated on the underlying asset dynamics with standard, developed and well known likelihood techniques. The same parameter values can be used to price options. Nevertheless this approach assumes that the risk neutral expectation necessary 
to price options is identical to the physical expectation. Relying on physical volatility parameter values to price options comes at the cost of poor pricing performances. The divergence between risk neutral and physical volatility parameter values motivates option pricers to calibrate models directly on option prices. However the econometric frameworks available for estimating volatility models directly on option prices are less developed than their time series counterpart. Chapter two and three of this dissertation develop and enhance the methodologies related to model and data selection in this context.

In chapter two, I assess and study the effect of measurement, model and parameter uncertainty on option pricing model performance evaluation. Parameters estimated on option prices, resulting predicted prices and loss functions are only point estimates and are characterised by an entire probability distribution function. This dissertation provides a framework to obtain the loss function distribution instead of relying only on a single point estimates as commonly done in practice and industry The measured estimation uncertainty and uncertainty around option pricing models' loss function is large. Furthermore, I find that over fitting is a concern for models calibrated on a single cross-section of options. Cross-sections of options display wide heterogeneity both in term of pricing performance and estimation uncertainty. This heterogeneity has to be accounted for when continuous recalibration is used.

In chapter three, I focus on option data selection and answer the question: What is the impact of data selection on model-predicted option prices? Alternative and divergent data selection methods are presented in the literature and in practice. I demonstrate that parameter estimates and predicted prices are largely affected by the data used for estimation purposes. Filtered data and the loss function interact and provide a higher implicit weight to certain options. The option prices excluded from the calibration sample are not well captured by the resulting parameter estimates. We show that in addition to model selection and loss function selection, data selection should be regarded as a key determinant of option predicted prices.

\section{Impled volatility and realized volatility}

In the second part of the dissertation, I focus on the difference between options implied volatility and realized volatility. The risk neutral volatility expectation surpasses the physical volatility expectation because of the volatility risk premium. For example, since 2002, the VIX is more than $3.5 \%$ higher the than the S\&P 500 volatility. Investors pay a premium to hedge against an undesirable change in spot volatility. In chapter four and five, the dissertation highlights that the discrepancy between implied and realized volatilities is beneficial in the case of asset pricing but is impairing in the context of risk management.

In chapter four the dissertation studies the expected returns of the cross-section of stocks in the US market. We find that option implied information helps to explain the dispersion in expected return across stocks. More precisely, the difference between realized and implied 
volatilities is a priced factor on the equity market. Stocks which co-move with the volatility risk premium are compensated with higher expected returns. In this context, the spread between implied and realized volatilities is a valuable source of information to indicate the state of the world for equity investors. On the one hand, equity index option implied volatility is relevant for the cross-section of the entire universe of stocks. On the other hand, oil implied volatility is only relevant within oil related industries. Our finding supports the theory of market segmentation and investors' limited attention.

In chapter five, the dissertation assesses the risk management application of implied volatility. We study, evaluate and compare the performance of implied volatility based Value-at-Risk (VaR) measures for three major equity indices over a period of up to twenty-four years. Our results demonstrate that the volatility risk premium embedded in option prices causes implied volatility $\mathrm{VaR}$ to be downwardly biased. In an attempt to correct for this impeding factor we provide a parametric and a non-parametric volatility risk premium adjusted implied volatility VaR. The adjusted risk measures correct efficiently the implied volatility VaR bias. But they do not outperform a standard time series VaR such as the GJR Garch VaR. We conclude that unless more sophisticated volatility risk premium adjustments are developed, option implied volatility has limited value when predicting VaR. 



\section{Addendum}

Options and derivatives are, by definition, instruments of high social value. In $350 \mathrm{BC}$, options were already being written in order to satisfy the social need to manage, to exchange and to hedge risk. It is interesting to note that the option market only reached maturity once mathematical methodologies were developed to price options and once the option dynamics was understood. Indeed,the option market has exploded since the development of the first mathematical formula for option pricing.

In 1998 the Over-The-Counter derivatives total notional amount outstanding was slightly more than $\$ 72,000$ billion. By 2013, the total notional amount outstanding increased to more than $\$ 710,000$ billion. Due to its size, the derivative market became increasingly relevant for the world economy. By comparison, the world gross domestic product is estimated by the World Bank at $\$ 72,000$ billion. However, the size, the fast pace of innovation and the complexity of derivative products have led to among the greatest corporate and social turmoil. Some of the biggest world corporations like Metallgesellschaft, Société Générale and Morgan Stanley lost billions of dollars because of the misunderstanding and mismanagement of derivatives. Usage of derivatives can backfire and result in dire consequences.

This is evidence that practices on derivatives market are still sub-optimal. Creating knowledge helps develop sound practices and decisions on option market and reduces the likelihood of unexpected financial catastrophe. In this thesis, we provide new methods and frameworks to improve upon risk management practices in option markets. We improve and rationalize the following processes required when dealing with option markets: Model Selection, Model Estimation, Option Based Risk Measurement. Additionally, we also show how new sources of risk, such as oil price uncertainty, need to be considered by corporate risk managers.

The topics covered and the solutions offered are relevant for two non-academic communities. The risk professionals in the industry will benefit from the findings and the methodology we propose, helping them to make rational decisions. Option traders in trading companies, option hedgers in banks and commercial firms are concerned here. Moreover, any risk management institution or department is a target group for our methodological innovations, which reflect more accurately the true level of risk. The regulatory bodies in charge of the development and implementation of directives and regulations to promote financial stability are our second audience. The European Union through the European Securities and Markets Authority and European Market Infrastructure Regulation initiatives and the global Basel Accords 
emphasize the need to account for new sources of risk and for the dynamic aspect of risk. Our findings and approach respond to these needs.

Practitioners, such as option traders or risk managers, deal with option markets on a daily basis. However, these professionals face complex decisions such as selecting the appropriate model amongst the plethora of available pricing models, determining the value of the input in a model and selecting a source of data on risk. The majority of the practitioners have made these decisions based on general procedures, habits or rules of thumb. This thesis innovates and improves upon the current approach by providing a scientific framework to help decision making. Our methodology helps to quantify and to measure the benefits and drawbacks of alternative choices. Moreover, we evaluate different alternatives and provide recommendations on the alternative to adopt.

\section{Model Selection}

First, risk managers and options traders can apply our methodology to choose and select amongst alternative models accounting for model and parameter uncertainty. Previously, this additional source of risk was ignored. Users ran the risk of selecting more complex models but ignoring the additional uncertainty caused by this complexity. The approach we propose allowsfor measuring objectively this trade-off and offers a method to assess whether a model is selected only because of chance or because of actual good performance.

Moreover, we show that, contrary to common practice, the best model to use to price or to hedge derivatives is not necessarily always the same. It depends on the market conditions. The proposed methodology allows for selecting a model for a certain market condition. This competitive advantage of our approach is in line with the recent recommendations made by the European Union institutions in charge of financial stability. The notion of differentiating between a normal period and a stressed period for risk measurement is often mentioned in the guidelines of the European Securities and Markets Authority (ESMA).

\section{Model Calibration}

Secondly, model selection is closely linked to model estimation. Indeed, once a model is selected, proper parameter values should be used. The common practice is to use the parameter values that provide sensible predictions (option prices or option hedge). To do so, model parameters are estimated using option data. What data should be used to calibrate a model is still an open question. Every practitioner relies on different sources of data. Our assessment shows that the data selection process strongly impacts the predictions and implications of a model. This is the case for pricing options and hedging options. Accordingly, practitioners should be aware of the implications of selecting a specific type of option to calibrate a model. We provide a systematic way of evaluating the effect of a data 
selection process on predicted option prices. Following the procedure we have developed, one can in advance realize the implications for predicted prices. Our investigation shows that models are very sensitive to the input used. This is an important point to consider with respect to the new European Market Infrastructure Regulation (EMIR). This European initiative aims at improving stability on the Over-The-Counter derivatives markets. One key recommendation of this directive is to market-to-model non-liquid derivatives. Therefore, the value of the derivatives held by a bank, fund or firm reported in the balance sheet is defined by a model. Not only the model should be considered but also the input and the way parameter values are selected.

\section{Option based Risk Measures}

Thirdly, the frequency and severity of financial crises have dramatically increased over the recent decades. These crises acted as a wake-up call on the need for financial stability and better risk management practices. The major 1987 financial crisis was followed by the emergence of the first Basel Accords (Basel I). In 2004, the Basel II Accord was initially published. The Basel III Accord responded to the major 2008 financial crisis. The main mission of these accords is to ensure that banks and financial institutions save up sufficient capital to cover the potential losses of their risky activities. The amount of capital required of an institution is related to its level of risk. Measuring risk has appeared to be one of the most complicated tasks in the whole process. The JP Morgan risk measure, Value-at-Risk, has been adopted by practitioners and by regulators as the dominant methodology. This approach was even recommended by the Basel committee on Banking Supervision (Basel II). The success of the value at risk measure, that measures the loss that should not be exceeded with a cetain probability, is due to its intellectual simplicity. However, how to measure, or to predict, this $\mathrm{VaR}$ is still debated and is a challenging exercise. Relying on multiple measures of $\mathrm{VaR}$ is a reasonable alternative in order to obtain a certain range of potential losses.

Different models, such as volatility models, and different sources of information, result in different VaR estimates. It is therefore critical to understand the benefits and drawbacks of the alternative methodologies. It has been argued that traditional VaR measures suffer from a backward looking bias. These measures do not respond quickly enough to new market conditions and to an increase in the potential loss. Derivatives markets and options, on the other hand, are forward looking. The prices of these instruments depend on the investor's expectation of what will happen on financial markets in the upcoming days. Can option implied information be used to form VaR forecasts? Can option implied information VaR beat traditional VaR? These questions are relevant for any risk manager or regulatory body. In this thesis we answer these questions and highlight the limitations of option implied VaR, which explain why option implied VaR cannot outperform the traditional measures. Our conclusions are drawn from the results of different and complementary quantitative back-testing approaches. 


\section{Measuring Oil Price Uncertainty and its implication for the equity market}

The last part of the thesis turns to micro level risk management. Firms' internal risk managers need to assess and evaluate the alternative source of uncertainty the corporation is facing in terms of production cost, client base or cost of capital. Nowadays, one of these factors is very important: the price of oil. Indeed, a considerable number of firms are negatively affected by high and volatile oil prices. This topic is so relevant that the International Energy Agency (IEA) has organised a workshop dedicated to it. A report called 'Oil price volatility: causes, impacts and potential remedies' summarizes what is at stake for oil professionals. One of the major conclusions is that, despite the inherent nature of volatility, oil price volatility is undesirable for consumers and governments. Moreover, an important recommendation is to provide more information with respect to oil prices and oil prices forecast uncertainty. Reporting the level of uncertainty is beneficial for consumers. However, the proposition has been that this uncertainty can be measured by analysts. We know that analysts' recommendations and reports are biased in many ways. In this thesis, we study the oil market and provide an answer for this need to measure and account for uncertainty in oil prices. We rely on oil options data to form our measure. We observe important time variations in oil price uncertainty. This uncertainty is affected both by global macro-economic conditions and by oil specific events such as political tensions in the Middle-East. We evaluate the impact of uncertainty assumed by the oil professional analysts on firms and equity prices. We confirm the idea that uncertainty in oil prices has a negative effect on firm values. However, this effect is concentrated amongst firms in oil relevant industries. We even find some evidence that the sensitivity to oil uncertainty affects the firm's cost of capital. Based on our results, we suggest risk managers in oil relevant firms should closely monitor the evolution of oil uncertainty using option markets. If a firm's exposure to oil uncertainty is too substantial, it is advisable to manage this exposure viathe purchase of option contracts.

The results of this research have been presented on many occasions to different audiences in the formats of poster session (INFINITI 2013, Aix-en-Provence), colloquia (PhD Colloquium 2013-2014 Maastricht \& Luxembourg), peer-reviewed conferences (International Mathematical Finance Conference 2013, Miami; French Finance Association Conference 2014, Aix-en-Provence) in the academic environment, and professional presentation in an asset management firm (Robeco, 2014, Rotterdam). 


\section{Biography}

Gildas Yannick Blanchard was born on 25 August 1986 in Saint Renan, France. He holds a bachelor in business administration with finance from the Edhec Business School (Nice) where he was awarded the titles of 'Best Finance Student', 'Best Student' and 'Best Bachelor Thesis'. He also holds a first-class honours Bachelor in business administration with strategy from the University of the West of England (Bristol). In conjunction he worked in the banking industry (BNP Paribas and Crédit Agricole) as small and medium enterprise financial analyst and risk analyst. He obtained his Master of Science in Finance from the University of Durham in 2008 with a dissertation titled "Is Herding in capital markets generating a non-normal distribution of stock returns? An empirical investigation on the S\&P500 index".

In September 2010, Gildas joined the Finance department of the School of Business and Economics at the University of Maastricht. Gildas taught Risk Management to master students and coordinated the course Macroeconomics and Finance in the operational research and econometrics bachelor for which he was awarded the 'best teacher' of the finance department in 2012. His research has been presented both in academic and practitioners (The International Mathematical Finance Conference, INFINITI conference, French Finance Association Conference, The Australasian Finance and Banking Conference, Robecco) conferences.

Since September 2014, Gildas joined the Capital Markets department of Deloitte Luxembourg as Analyst for investment funds and alternative investment funds. 


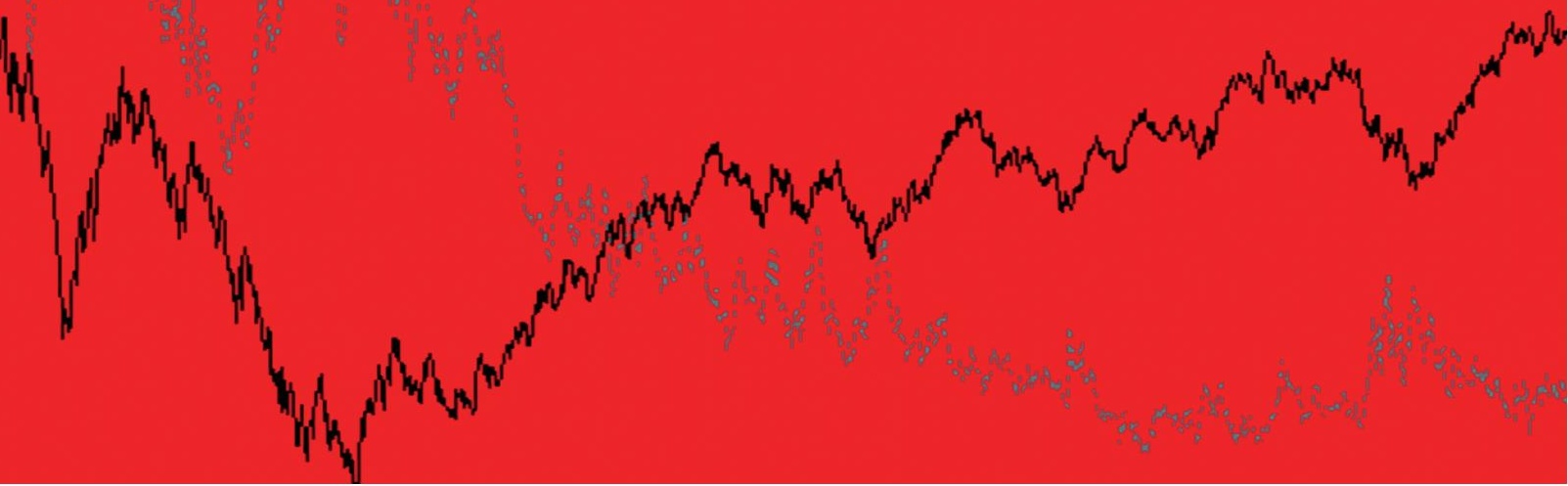

